\title{
UNDERSTANDING THE EFFECTIVENESS OF CROSS-CULTURAL VIDEO-MEDIATED COMMUNICATION
}

\author{
by \\ Selenge Nergui, MBA
}

\begin{abstract}
A thesis
submitted to the Victoria University of Wellington in fulfilment of the requirements for the degree of

Doctor of Philosophy
\end{abstract}

Victoria University of Wellington 


\begin{abstract}
People are increasingly using advanced communication technologies such as videoconferencing to collaborate across geographical boundaries and time zones. This presents problems because cultural values, attitudes, and behaviours influence how a given group of people perceives, understands, communicates and interprets information and knowledge.

This study explores how various factors including technology and cultural differences of participants affect their perceptions of the effectiveness of cross-cultural communication in videoconferences. The study identifies factors that most influence the effectiveness of cross-cultural video-mediated communication. It will help practitioners to: 1) make efficient use of resources while designing and facilitating videoconferences; and 2) incorporate cultural factors in assessing the effectiveness of cross-cultural distance learning events.

The study is situated in the Global Development Learning Network (GDLN) which spans the world with more than 120 distance learning centres and facilitates communication for development through videoconferencing technology. A multiple-case cross-cultural study has been carried out in GDLN affiliates located in four countries: Australia, Mongolia, New Zealand and Russia. Evidence for this case study comes from observations during videoconferences, semi-structured interviews with participants, documents and video recordings.

This exploratory study contributes to the body of knowledge in three research domains: development communication through the videoconferencing channel; cross-cultural factors in video-mediated communication; and perceived effectiveness of videoconferencing.
\end{abstract}




\section{Acknowledgements}

I wish to express my sincere gratitude to my two supervisors Dr. Brian Harmer and Alastair Smith. Thank you, Brian, for your guidance, patience, your generosity, your understanding in difficult times that I faced during this research, your warmth and wisdom, and your confidence in me. Thank you, Alastair, for your direction and constructive feedback provided often from a very different viewpoint into my research. Your firm belief in my ability to reach the goal of thesis completion was highly encouraging.

I wish to make a special note of appreciation for all those people in Australia, New Zealand, Mongolia and Russia who participated and contributed in the data collection phase of this study. There would not have been anything to talk or write-about, if not for your input; thank you very much for your time and support.

I'd also like to thank all those who have provided feedback for this work. Your time and support is much appreciated, thank you for your willingness to help and for your interest in my work.

A warm thank you to all my friends and colleagues at SIM who have made my journey with this study a very memorable one. I would not have made it this far, if not for your continuous moral support.

I'd also like to thank my family - my husband Bilegt and my three sons Tamir, Telmen and Tengis - for unconditional love and acceptance, for continuous encouragement you've shown throughout my long journey towards a PhD. 


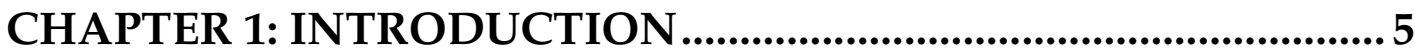

1. 1. Background of the problem and Research motivation.......................6

Cultural differences.................................................................................... 7

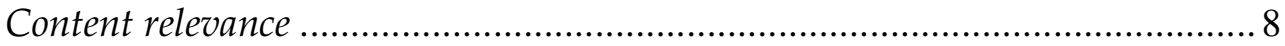

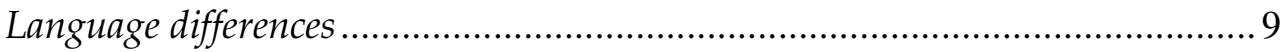

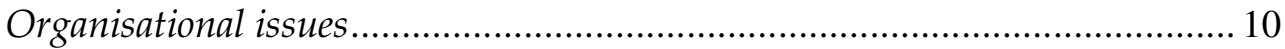

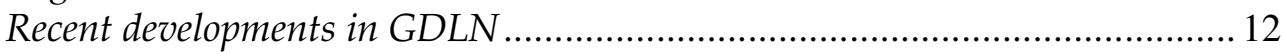

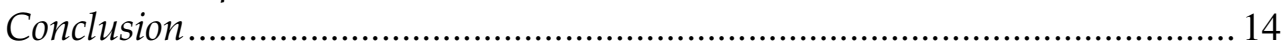

1. 2. The nature of the research problem and Research questions ............ 15

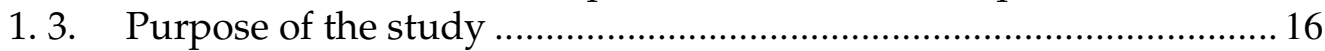

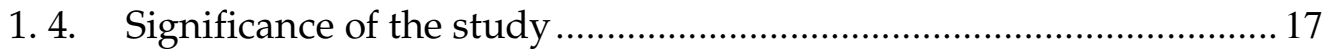

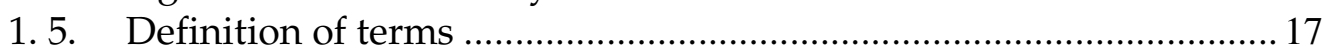

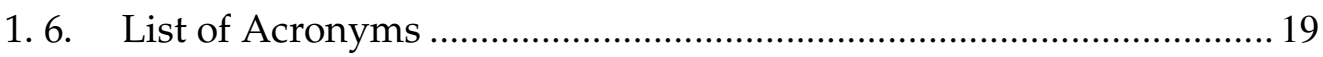

CHAPTER 2: LITERATURE REVIEW .............................................. 21

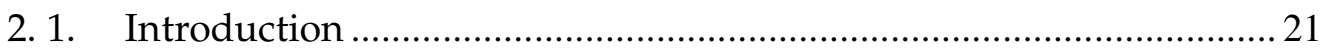

2. 2. Intercultural communication........................................................ 23

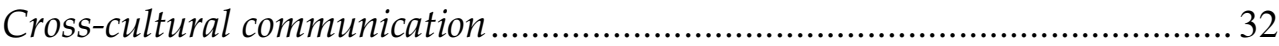

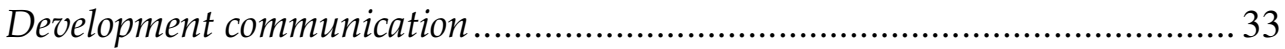

2. 3. Media Choice Theories ....................................................................... 40

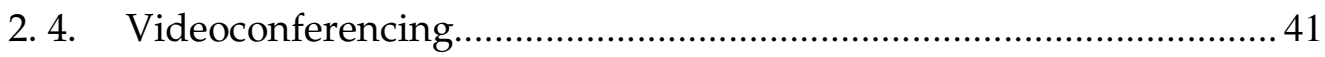

Videoconferencing in Distance Learning ................................................... 43

Videoconferencing in Organisational Communication .................................. 46

Videoconferencing in Development Communication ..................................... 47

2. 5. Perceived effectiveness of Videoconferencing ................................. 48

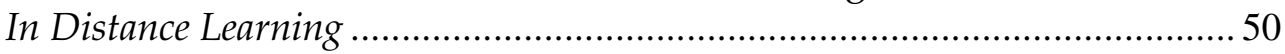

In Organisational Communication ....................................................... 51

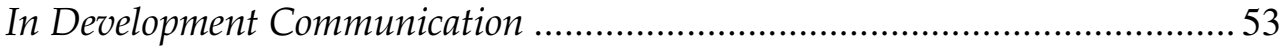

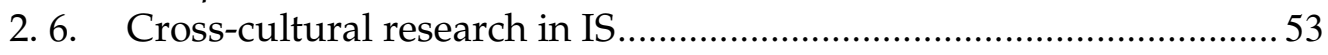

2. 7. Theoretical models informing my research ................................... 55

Cultural dimensions by Hall .......................................................................... 55

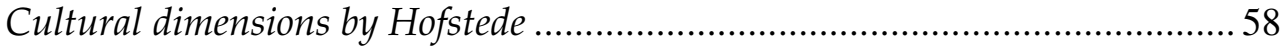

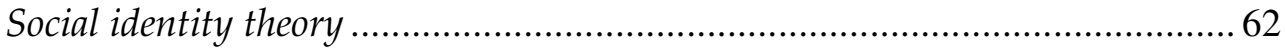

2. 8. Gaps in the knowledge and research questions ............................65 65

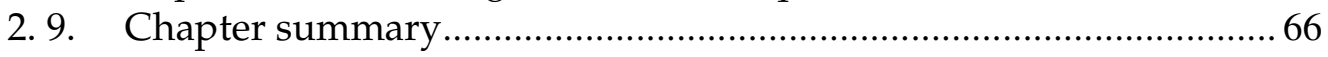

CHAPTER 3: RESEARCH METHODOLOGY...................................67

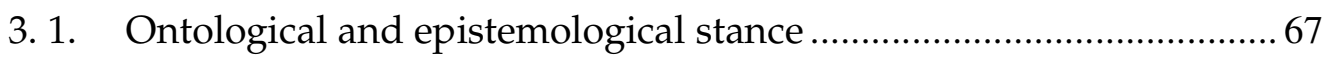

3. 2. The choice of an interpretive research philosophy ..........................69

3. 3. Rationale for the choice of case study ........................................ 71

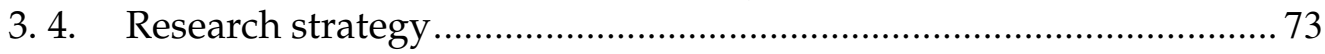

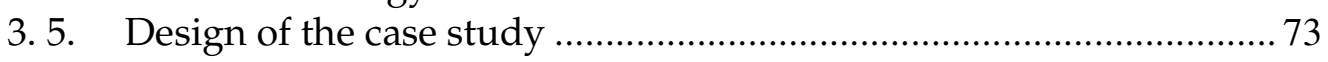




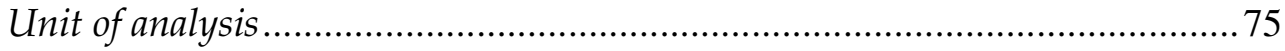

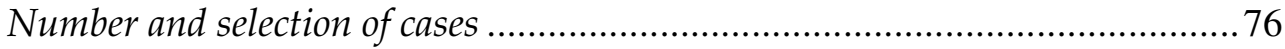

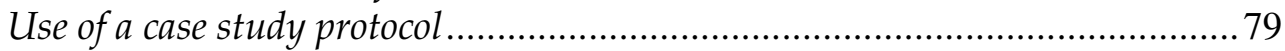

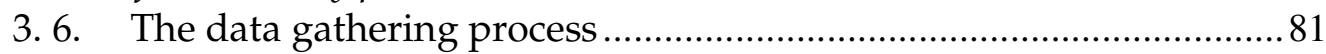

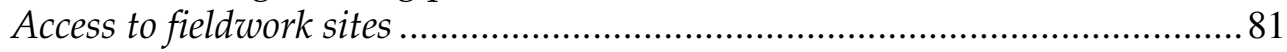

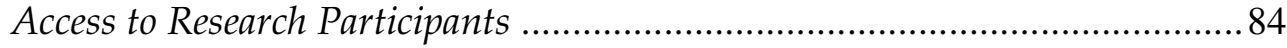

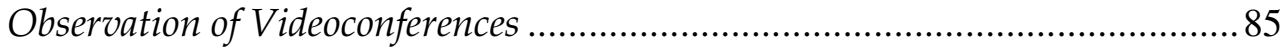

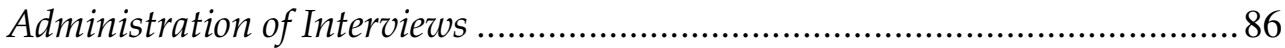

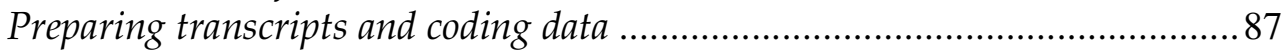

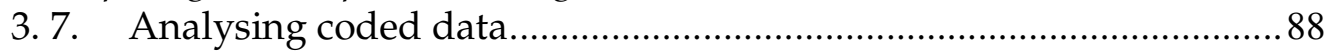

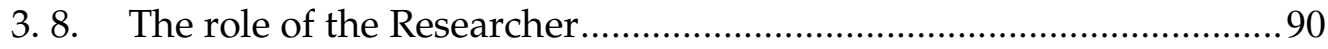

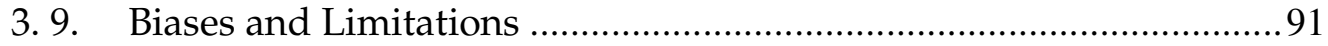

3. 10. Issues of Validity and Credibility.................................................... 94

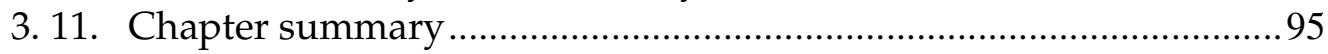

\section{CHAPTER 4: DATA INTERPRETATION AND PRELIMINARY}

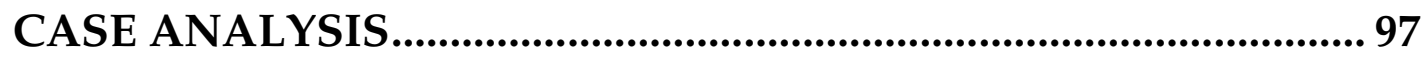

4. 1. The Australian Case ............................................................................ 97

Cultural differences of participants ............................................................. 98

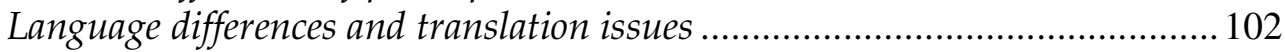

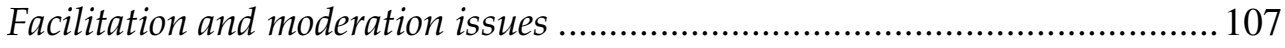

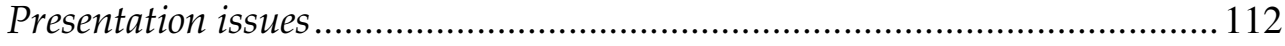

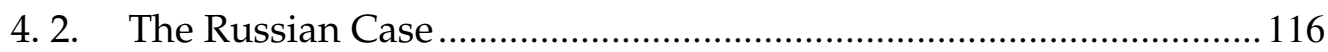

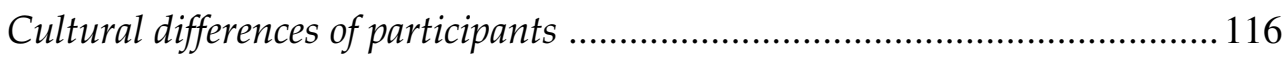

Language differences and translation issues ............................................. 124

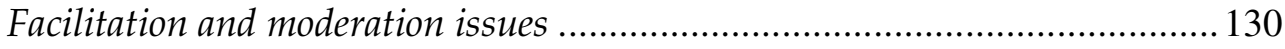

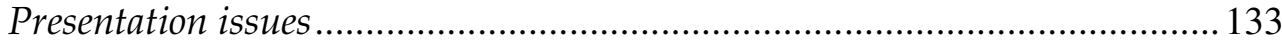

4. 3. The New Zealand Case .................................................................... 140

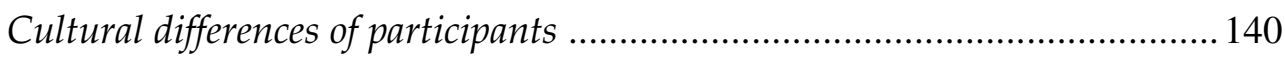

Language differences and Translation issues ............................................ 150

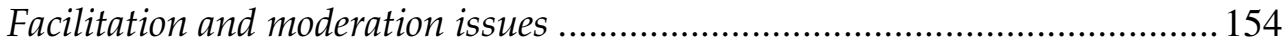

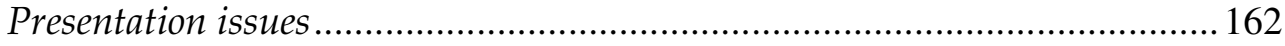

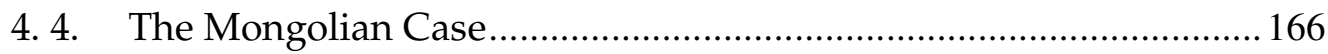

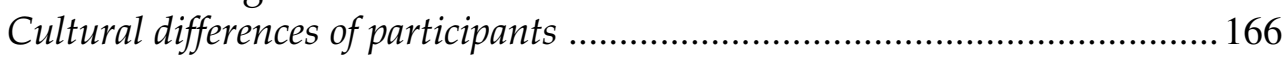

Language differences and translation issues ............................................... 176

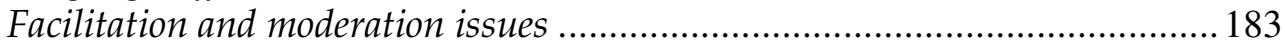

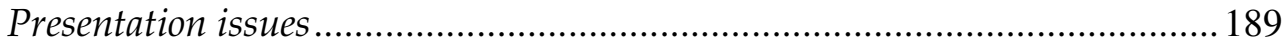

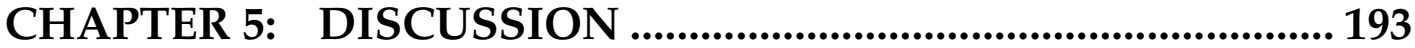

5. 1. Cross-cultural video-mediated communication on development... 193

5. 1. 1. Knowledge Transfer and Knowledge Sharing.................................. 194

5. 1. 2. Content of communication and its relevance ................................... 198

5. 1. 3. Nature of social interaction during videoconferences ........................201

5. 1. 4. Impact of Technology on Cross-cultural Communication ...................2203

5. 2. Impact of Cultural Differences of participants on communication. 205

5. 2. 1. Cultural diversity of participants: opportunity or obstacle? 
5. 2. 2. Impact of occupational and organisational culture ........................... 212

5. 2. 3. Knowledge and experience gap ................................................... 213

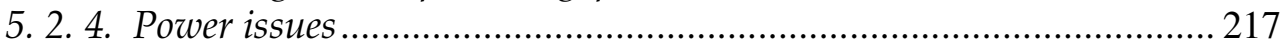

5. 3. Language Differences and Interpretation ...................................... 219

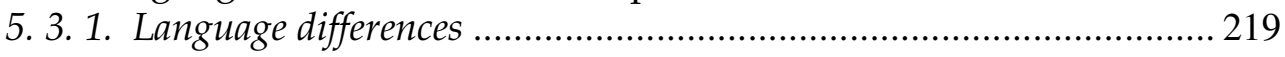

5. 3. 2. Translator/Interpreter as cultural mediator ................................... 220

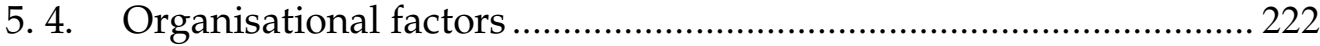

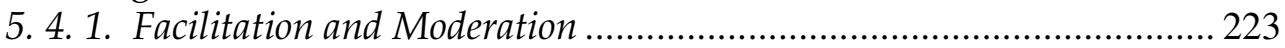

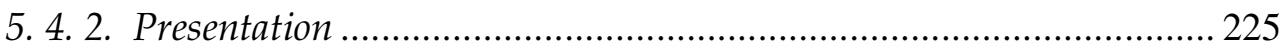

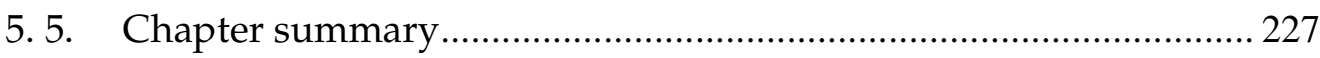

\section{CHAPTER 6. CONCLUSIONS AND IMPLICATIONS .............. 228}

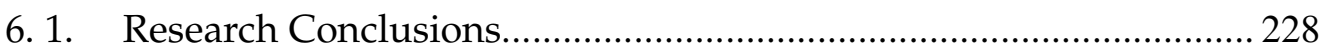

6. 1. 1. Cultural diversity and videoconferencing ...................................... 229

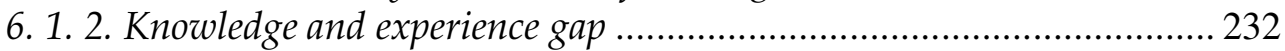

6. 1. 3. Role of technology and Videoconferencing culture ............................ 233

6. 1. 4. Role of the simultaneous interpreter ................................................. 234

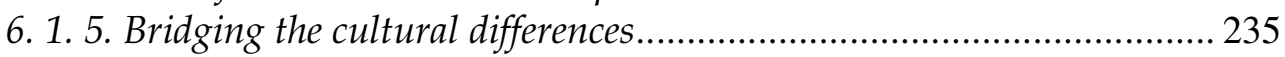

6. 1. 6. Verification of theoretical models informing this research ..................236

6. 2. Implications for future research ................................................... 237

6. 3. Recommendations for practitioners............................................ 238

REFERENCES: ....................................................................... 240

ANNEX A: INFORMATION SHEET FOR INTERVIEWEE ............. 256

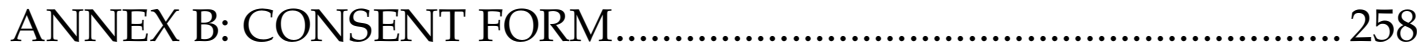

ANNEX C: INTERVIEW QUESTIONS ............................................. 259

ANNEX D: SAMPLE CLASSIFICATION OF NODES ....................... 262

ANNEX E: SAMPLE TABLE OF COMPARATIVE ANALYSIS....... 263

\section{LIST OF FIGURES}

Figure 1 Growth of Global Development Learning Network ....................... 13

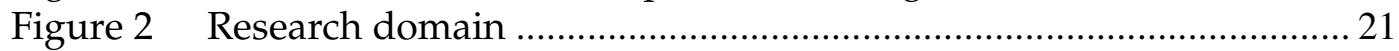

Figure 3 Influence of Culture on Behaviour and Behaviour on Culture ........ 25

Figure 4 Levels of cultural group membership of communicators................29

Figure 5 Examples of social contexts in Intercultural communication ........... 30

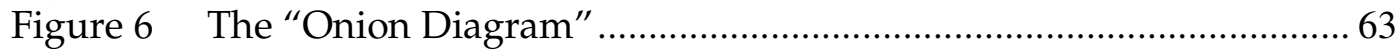

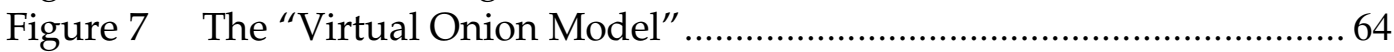

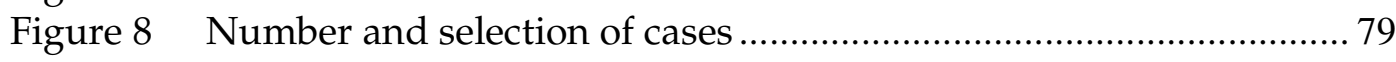




\section{LIST OF TABLES}

Table 1 The changing communication environment .................................. 37

Table 2 Hall's dimensions from videoconferencing perspective ...................58

Table 3 Hofstede's dimensions from videoconferencing perspective...........59

Table $4 \quad$ Hofstede's response to the criticism of his model.............................61

Table 5 Cultural differences and similarities at national and organizational

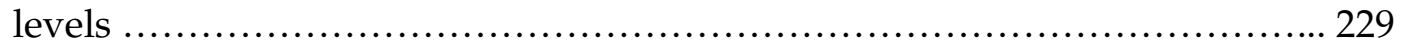




\section{Chapter 1: Introduction}

The use of video-mediated communication has grown far beyond facial framed transmissions for video telephones and people are expecting more and more from available technology. Economy of scale has made videoconferencing an affordable medium of choice because it precludes travel and is accessible on demand (Olaniran, 2009). Governments, businesses, universities and international organisations are using videoconferencing systems to make possible meetings that otherwise just would not happen and to increase the efficiency of communication across geographic boundaries. Business people in multinational environments deal with a variety of situational barriers and cultural differences, such as time zones, languages, and common practice differences (Ferry, Kydd, \& Boyles, 2012). Government agencies use national videoconferencing networks to communicate with provincial affiliates for conducting meetings during which they update ongoing projects and make important decisions. Universities deliver distance learning courses to remote domestic and international students or full-time working professionals using videoconferencing in conjunction with other online learning systems. Multinational corporations use videoconferencing for instant communication with business units and clients in different locations to coordinate decision making processes and operate in cost-effective and timely manner. International development agencies increasingly employ videoconferencing for sharing information and knowledge with developing countries as well as for operational communication and training of staff based at field offices around the world.

Videoconferencing can be point-to-point - connecting just two sites; or multipoint -connecting several sites at once. Videoconferencing is synchronous, i.e. the system is used at the same time by all sites connected to the session and hence, the participants need to be simultaneously present at the joined locations for communication to occur. The synchronous nature of videoconferencing makes it a highly appropriate technology for education, information sharing 
and organisational communication. Videoconferencing can be desktop-based, when participants use computer and see the image of their counterparts on its monitors or room-based, when participants are seated in a room facilitated by videoconferencing equipment and see the connected sites on a large single or dual screen. Communication via videoconferencing can be translation-mediated if participants at connected locations speak different languages.

Even though much has been written on perceived effectiveness of computermediated communication in distance learning and organisational communication context, little attention has been devoted to perceived effectiveness of cross-cultural video-mediated communication in the global knowledge sharing context.

\section{1. Background of the problem and Research motivation}

Critical description of the social and historical background of the research setting helps the intended audience to understand how the current situation under investigation emerged (Klein \& Myers, 1999).

The research questions arose from my past experience as a training coordinator for the Distance Learning Centre (DLC) in Ulaanbaatar, Mongolia - part of the Global Development Learning Network (www.gdln.org). GDLN is a global partnership of learning centres that offer the use of technologies, tools and services developed in the field of distance learning to connect people and organisations working in international development around the world (World Bank, 2009b). Much of what is written on the strategy of development is produced by people whose point of view is from the outside looking in, that is, from the perspective of specialists from the developed world who diagnose and prescribe for a client country people whose real-life experience they have not shared (Fisher, 1997). In this case, the point of view is from the inside, as it is based on problems experienced during three years of my working with GDLN. My research is basically inspired by the dynamically evolving expansion of the network.

Klein and Myers (1999) proposed a set of principles for conducting and evaluating interpretive research in information systems. A description of what 
is interpretive research in information systems will be presented in Chapter 3 of this thesis, but the set of principles for its conduct is mentioned here to justify the extended explanation of motives that inspired me to undertake this study. Information provided in the following chapters ensures application of the contextualisation principle from the set of principles proposed by Klein and Myers (1999).

In order to make my story understandable to the readers, I analysed the problems that I experienced during three years of involvement in videoconferencing and identified the following four categories:

- Cultural differences among participants

- Content relevance

- Language differences

- Organisational issues.

Based on the discussions held during East Asia and Pacific (EAP) regional meetings of DLC managers and on routine communication related to GDLN activities, I concluded that larger community of my colleagues were concerned by similar problems.

\section{Cultural differences}

One videoconferencing event can connect audiences from several different cultures and that is often a case in activities of GDLN, spanning the whole world with more than 120 DLC's in six regions: Africa, East Asia and the Pacific (EAP), Europe and Central Asia (ECA), Latin America and the Caribbean (LAC), the Middle East and North Africa (MENA), and North America. Programs are targeted to one region at a time in order to fit into a particular time zone as well as to facilitate the translation issues. As Mongolia belongs to the East Asia and Pacific region of GDLN, the countries Mongolia DLC usually joins in the same program include, but are not limited to Australia, China, Vietnam, Indonesia, Korea, Thailand, Japan, Papua New Guinea, East Timor, Singapore and Philippines. The content is delivered from GDLN studios in Washington DC as well as from Canberra, Tokyo, Singapore or Seoul. 
Occasionally, Mongolia DLC connects to the videoconferences targeted to the DLCs in the Russian Federation (ECA region).

Based on the above, it is evident that a number of different cultures get together for a GDLN event to share and exchange new information and knowledge. Cultural values, attitudes, and behaviours significantly influence how a given group of people views, understands, and communicates information and knowledge (Pauleen \& Murphy, 2005). Thus, there is a need to promote cultural differences and learn from them, not seek to eliminate or ignore them (Pauleen, Evaristo, Davison, \& Ang, 2006).

\section{Content relevance}

In October 2002, the World Bank, in partnership with the Australian Agency for International Development (AusAID), commissioned the Australian Council for Educational Research (ACER) to conduct a survey to assess the knowledge needs of existing and planned DLC's in ten countries in the EAP region. The report findings suggested that the most important reasons for learning for the clients were to develop practical and broad conceptual understanding in five knowledge areas that were identified as being of the highest priority: government and civil society; policy and administrative management; education; banking and financial services; and ICT skills (Ainley, Banks, \& Croger, 2002). By the same year, more than eighty percent of the programs delivered via GDLN were offered by the World Bank Institute (WBI) - the learning arm of the World Bank Group (Lorenzo, 2002) compared to the later

data indicating that sixty four percent of all activities are initiated by organizations other than the World Bank (Weber-Fahr, 2006). The majority of this content consists of training programs repackaged from existing face-to-face courses to distance learning mode.

WBI learning programs predominantly focus on knowledge sharing and awareness raising among stakeholders by facilitating access to knowledge and exchange of information (World Bank, 2009c). WBI also develops courses designed to meet the learning needs expressed by clients and partners by offering country-specific programs tailored to immediate as well as longer-term 
capacity-building needs. Among factors that adversely affect the potential of information and communication technologies to address poverty is affordability of the solutions and the fact that most content is of questionable local relevance (Ryan, 2003). The topics for the courses to be offered through GDLN are selected predominantly on the basis of their perceived importance from the standpoint of the World Bank and other donor institutions, and such top-down approach was one of the major issues that many participants of GDLN events unequivocally and passionately criticize (Assié-Lumumba, 2008).

\section{Language differences}

Training courses delivered from GDLN studios in Washington DC are conducted in or interpreted into six official languages recognised by the UN: Arabic, Chinese, English, French, Russian, and Spanish. Therefore, translation and interpretation is not an essential problem for DLCs in some regions: Spanish is an official language in most of the countries in Latin America and the Caribbean region just as French is in many African countries. However, countries in the East Asia and Pacific region all speak in their own languages (Mongolian in Mongolia, Vietnamese in Vietnam, Japanese in Japan, Korean in Korea, Thai in Thailand and so on) therefore each DLC should individually arrange for translation of written materials and simultaneous interpretation of communication during the actual videoconference.

The majority of the targeted audience in Mongolia have little or no English language skills, thus most of the videoconferencing events involve written translation of the training materials and oral simultaneous interpretation of actual session in light of limited connection time. Written translation is not a huge problem as appropriate people with good English language skills can easily be found, given a good per/page rate and adequate timeframe for their services. However, there aren't many skilled simultaneous interpreters in Ulaanbaatar and it is practically impossible to find the one who is an expert in the particular topic of the videoconference. Obviously, the level of accuracy during simultaneous translation is much lower compared to consecutive translation. Interpreting is a highly complex cognitive and communicative task. 
Every language organizes meaning differently, and trying to find direct equivalents in two languages often leads to nonsensical results (Mikkelson \& Willis, 1993). When it takes place in a videoconference setting, the technological challenges and remoteness add yet another dimension of complexity and difficulty (Braun, 2007). Moreover, imperfect interpretation of specific terms and definitions during GDLN sessions worsens the situation by creating misunderstanding and confusion for participants at the remote sites.

\section{Organisational issues}

\section{Facilitation}

In discussing Internet-based education, Berge and Collins (1995), identified four facilitator roles: 1) technical - ensuring comfort with the technology, 2) social creating a friendly environment, 3) managerial - administering and organising, and 4) pedagogic or educational - ensuring that the educational task is accomplished. These roles apply to videoconferencing. Inexperience, poor preparation and planning, unsuitable teaching strategies and inefficient training on the part of facilitator have a bearing on the quality of teaching and learning in a videoconferencing setting (Knipe \& Lee, 2002).

In the GDLN context, facilitation at the event initiating side includes responsibilities connected with: designing a course that is appropriate to the targeted audience; offering content that is relevant to their needs; producing course materials that will motivate participant's interest; inviting effective presenters and providing them guidance on the use of technology; ensuring follow up actions, course evaluation, etc. Facilitation at the participating DLCs side consists of activities to be carried out before, during and after the videoconferencing event. Preparation period would include: identification of individual participants; timely distribution of program agenda and course materials; contracting translators and interpreters; preparing copies of training materials, etc. On the day of the event, the facilitator should welcome and register the participants, briefly explain to them the use of relevant equipment (headsets and microphones) and ensure smooth coordination between the participants, moderator or a chairperson, simultaneous interpreter and technical staff. After the event, the facilitator ensures that the participants fill 
the evaluation forms and organises follow up actions if any. Successful interaction between remote participants in a videoconference setting may often critically depend on the expertise of a facilitator (Hedestig \& Kaptelinin, 2005).

\section{Moderation}

The moderator for a videoconference is essentially the same as a chairman for a meeting. Moderating would therefore have a meaning similar to that of chairing a meeting. Sometimes, a term 'Site Facilitator' is used in distance learning context referring to a person who carries out duties similar to that of a moderator (Sherry, 1996).

The key roles of a moderator for a videoconference include: introduce all speakers, panellists, resource persons and VIP guests; keep the event flowing smoothly by managing the floor or in other words, making all the transitions from site to site and person to person; manage the time according to the event agenda; make the videoconference interactive by ensuring that participants are recognized and given the opportunity to speak/interact; facilitate the communication by eliciting questions and comments and providing summaries during and at the end of the session to highlight important points.

In a videoconferencing event, as in any other official meeting, only one participant can speak or "have the floor" at any point in time, therefore control of the floor or how the floor is taken, lost or granted is crucial for a meeting's process and outcome (Dustdar \& Hofstede, 1999). The moderator at each site should be the one responsible for this task and in doing so be tactful and considerate, especially in the event that the conference involves participants from different cultures.

From my own observations, I conclude that a moderator with good communication and time-management skills can make the best use from a videoconferencing event whereas even a very interesting program designed specifically for a particular audience could become just a waste of time to the

participants if there was a lack of adequate chairing of a videoconferencing session. 


\section{Presentation}

A distant graduate student from California says: "having the occasional professor who is uncomfortable with the technology can make life difficult on the other side of the screen. It's hard when professors are a little bit technologically challenged" (Olsen, 2003). Likewise, many presenters in GDLN events feel uneasy in front of the camera and especially when they see several sites connected and many senior government officials are present in each country's videoconferencing room. This uneasiness or 'user apprehension' is one factor generally overlooked when assessing the effective use of new communication technologies, including videoconferencing (Campbell, 2006). Challenged not only by the technology but also by the questions and comments from remote centres, the presenters oftentimes appear nervous and unfocused.

One of the commonly cited downsides of videoconferencing is the difficulty of sustaining the interest of the remote learners and lack of specific training and guidance for presenters (Martin, 2005). The presenter becomes just a "talking head" when he/she continues speaking for too long. Therefore, it is advisable that presenters spend some time preparing for a videoconference: participate in a video session as an observer to understand the flow of the process; learn how to use the equipment and maintain eye contact with the camera; practice their own presentation skills; learn how to work with the interpreters; find out more information about expected participants; plan for interaction within and among local and remote sites; consult with relevant people regarding the desired "dress code for videoconferencing"1 and so on (World Bank, 2006).

\section{Recent developments in GDLN}

Since its launch in June 2000, GDLN has rapidly developed from 11 affiliates to 115 Affiliates in 2006 (see Figure 1) but since then growth has slowed down. Currently, the GDLN is a partnership of over 120 recognised institutions around the world (World Bank, 2009c). Affiliates - learning centres in more than eighty countries worldwide - enable their clients to communicate with

\footnotetext{
${ }^{1}$ In a videoconference, participants are advised to wear pastel and light-dark combinations and avoid red, black and solid white, large prints, shiny jewellery, etc.
} 
each other for knowledge sharing and stakeholder engagement events in a timely and cost-effective manner.

Figure 1 Growth of Global Development Learning Network (modified from Weber-Fahr, 2006)

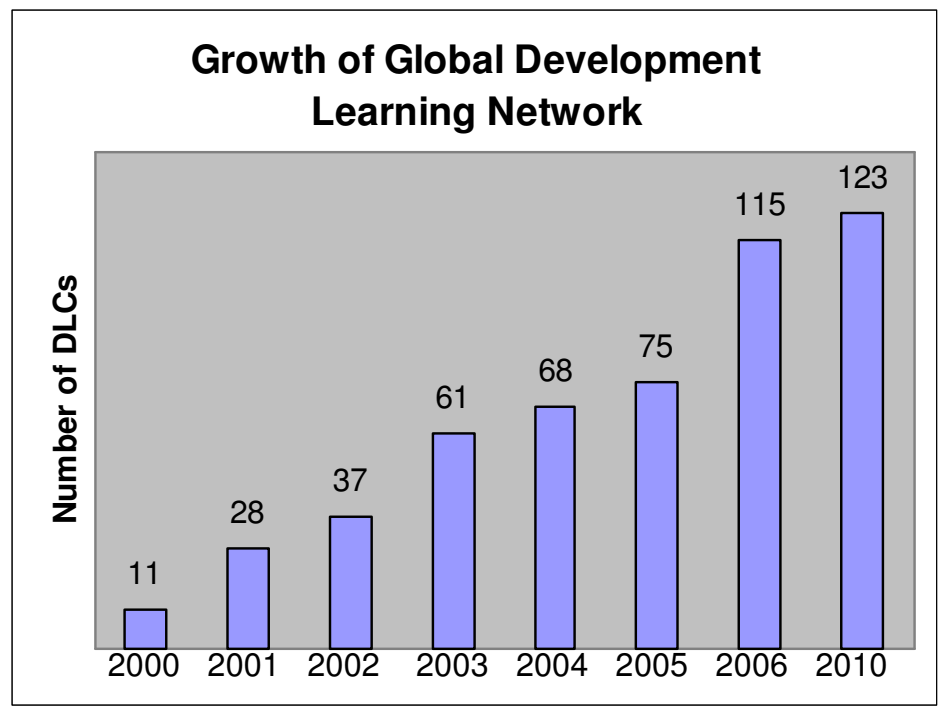

Note: GDLN Affiliates include all organizations signing a GDLN Network Agreement.

The signing of a cooperation agreement between the GDLN and JICA-Net, a distance technical cooperation project promoted by Japan International Cooperation Agency (JICA) - in November 2004 resulted in the world's largest development learning network, with a combined reach of more than 205 centres in 93 countries (JICA-Net, 2009; Tokyo Development Learning Center, 2004). The Japan - World Bank Distance Learning Partnership Project, known as the Tokyo Distance Learning Centre (TDLC) Project, comprising of the state-of-theart studio facilities was launched in the same year. The TDLC acts as a hub for the East Asia and Pacific region of GDLN. The joining of hands between the GDLN and JICA-Net means better and more widespread connectivity, establishing the foundation for an innovative range of knowledge sharing activities (Tokyo Development Learning Center, 2004). Both networks can share their distance learning contents to reach a much wider audience. JICA's extensive experience in the field benefits WB operations while allowing Japan's development experience to be disseminated more widely through the GDLN centres. 
With increasing links to in-country networks, GDLN has been contributing to the knowledge sharing agenda by extending its reach to more than 1000 access points around the world (World Bank, 2009b). For example, in 2007 the GDLN was joined by China Western Development Distance Learning Network (CDDLN) that includes the management and bridging hub based in Beijing and 11 DLCs most of which are located in the poorer western provinces of China (World Bank, 2009a). Other networks that form part of GDLN include the Association of African Distance Learning Centres (http://www.aadlc.com/) with 15 centres in African countries.

GDLN organises joint dialogues and learning activities in partnership with other multilateral and bilateral development agencies such as the Asian Development Bank (ADB), United Nations organisations such as UNDP and ILO, Japan Bank for International Cooperation (JBIC) and European Bank of Reconstruction and Development (EBRD).

The School of Government of Victoria University of Wellington (VUW) and the Centre for Continuing Education and Executive Development are part of GDLN since 2007. The Distance Learning Centre at the VUW aims to develop into a hub for New Zealand in order to facilitate sharing of this country's knowledge and experience on development issues with the rest of the world via GDLN. VUW's permanent videoconferencing facilities are located in the premises of the Central Library on Kelburn campus and by the Commerce Library on the Pipitea Campus. In addition, VUW's lecture theatres can be utilised for videoconferencing events involving larger audiences.

\section{Conclusion}

The above discussions on the main problems experienced during my work with GDLN and recent developments in the network activities are intended to provide contextual information that is important for better understanding of the subject matter.

The four problem categories described above are directly relevant to the factors that influence the perceptions of participants for the effectiveness of videoconferences. Having acknowledged the existence of these factors, my 
research focused on cross-cultural communication factors that influence the perceived effectiveness of communication that occurs during videoconferences. GDLN's rapid growth and fostering partnership as well as the VUW's collaboration with this network explains the logic behind the chosen research method and project design that will be described later in this document.

Researchers are urged to explore the ways in which videoconference-mediated collaboration - that would otherwise not occur - fosters significant economic, personal, or professional growth and mutual understanding among people (Greenberg, 2004).

\section{2. The nature of the research problem and Research questions}

This study focused on the issues of effectiveness in cross-cultural communication that occurs through the videoconferencing channel rather than effectiveness of existing videoconferencing systems. It identified and analysed factors that influence the effectiveness of cross-cultural communication mediated by videoconferencing systems. The nature of cross-cultural videomediated communication calls for a closer look into the issue of cultural differences among the participants and the challenge of bridging these differences for more effective interaction (Pauleen, Evaristo, Davison, \& Ang, 2006).

Videoconferencing proved to be more effective for courses designed to teach "knowledge" rather than "skills" (Wisher \& Curnow, 2003). The objectives of GDLN are changing in the same direction: if the key strategic drivers in 2000 were focused on building skills in the public sector by enhancing core competencies of people and teams, the priority now has shifted towards knowledge sharing and stakeholder engagement (Weber-Fahr, 2006). In the GDLN context, knowledge sharing involves mobilisation of external and local agencies to enable exchanges for accelerated implementation of development projects whereas stakeholder engagement means interaction for policy formulation upstream and during project/program work through dialogue and partnerships (Global Development Learning Network, 2005). Therefore, this research focused on the content that is comprised of learning programs and 
communication events aimed at knowledge sharing and stakeholder engagement rather than training programs aimed at skill-development.

This research attempted to identify the factors that influence perceived effectiveness of videoconferencing in the context of GDLN which facilitates cross-cultural communication of people working in development around the world. The aim was to compare the effectiveness of videoconferencing as perceived by the participants of GDLN events. The study then identified the common factors that influence their perceptions. Particular emphasis was given to the factors that are associated with cultural differences among the participants.

Following the discussion on the purpose and objectives of the study, the primary research question was formulated as follows:

\section{What cross-cultural communication factors influence the effectiveness of videoconferencing on development issues as perceived by the participants?}

Given the cross-cultural nature of the communication under the study, this research also sought to discover whether cultural differences among the participants influence the effectiveness of videoconferences. Consequently, the secondary questions were formulated as follows:

- How do the cultural differences influence the effectiveness of videoconferences in perceptions of participants?

- How can the factors identified be used to bridge these cultural differences for more effective communication?

\section{3. Purpose of the study}

Identification of factors that influence the effectiveness of cross-cultural videoconferencing, especially those associated with the cultural differences of participants, will help practitioners to: 1) effectively use the knowledge and resources while designing and facilitating videoconferences; and 2) incorporate cultural factors in assessing the effectiveness of cross-cultural videoconferencing on development issues. In addition, the research aimed to 
suggest how to use various cross-cultural communication factors in bridging the cultural differences for more effective communication.

\section{4. Significance of the study}

This thesis aimed to respond to calls for more in-depth and innovative crossculturally framed information systems research exploring the impact of cultural differences on the use of information and communication technologies (Hunter, 2001; Myers \& Tan, 2002). It contributed to the body of knowledge in the development communication domain by studying the largest videoconferencing network that facilitates cross-cultural communication for development. This research thus reacted to recommendations of scholars to investigate the usage of videoconferencing technology in the exchange of content on development issues and understanding the nature of discussion and practice within a development network (Wilkins \& Mody, 2001).

The findings of this study not only contribute to the understanding of perceived effectiveness of video-mediated cross-cultural communication among academics, but also provide helpful insight for practitioners. For instance, every GDLN activity is a result of joint efforts of various teams working together across time and distance and it costs thousands of dollars that are spent on connectivity and technical support, instructional design, management and administration, translation and so on. By using human and financial resources efficiently, GDLN or any other videoconferencing network can facilitate more efficient and effective interaction within the network.

The enquiry revealed and acknowledged that cultural differences influence the effectiveness of communication and identified the common factors that affect the video-mediated communication for development.

\section{5. Definition of terms}

Terms and concepts which occur frequently deserve an explanation as to the meaning attributed to them in this dissertation. They are listed in alphabetical order below. 
Communication involves the exchange of meaning between individuals and includes individual's behaviour which is perceived and interpreted by others (Adler, 2002)

Communication effectiveness refers to the extent that individuals are able to minimize misunderstandings (Hubbert, Gudykunst, \& Guerrero, 1999). In other words, communication effectiveness in terms of whether the receiver understands the message as the sender intended.

Cross-cultural communication involves comparisons of communication across cultures (Gudykunst, 2002)

Culture is "the collective programming of the mind which distinguishes the members of one human group from another ... the interactive aggregate of common characteristics that influence a human group's response to its environment." (Hofstede, 1980', page 25). Culture influences communication but is also constructed and enacted through communication (Martin \& Nakayama, 1999)

Development is defined as a widely participatory process of social change in a society, intended to bring about both social and material advancement for the majority of people through their gaining greater control over their environment (Rogers, 1976).

Development communication is a social process based on dialog using a broad range of tools and methods. It is also about seeking change at different levels, including listening, building trust, sharing knowledge and skills, building policies, debating, and learning for sustained and meaningful change (World Bank, Food and Agriculture Organization of the United Nations, \& Communication Initiative, 2006).

Diversity is defined as cultural differences in values, beliefs, and behaviours learned and shared by groups of interacting people defined by nationality, ethnicity, gender, age, physical characteristics, sexual orientation, economic status, education, profession, religion, organizational affiliation, and any other grouping that generates identifiable patterns (Bennett \& Bennett, 2001) 
Intercultural communication - communication between persons of different cultures (Hall, 1959)

Intercultural competence is the ability to communicate effectively in crosscultural situations and to relate appropriately in a variety of cultural contexts (Bennett \& Bennett, 2001)

Organisational culture is defined as shared perceptions of organisational work practices within organisational units that may differ from other organisational units (van den Berg, 2004). Organisational culture' is used in this thesis as a comprehensive term, encompassing national, occupational and corporate culture.

Perceived effectiveness of videoconferencing - participant's view regarding the effectiveness of communication that occurred during a videoconference (see term "Communication Effectiveness" above)

Videoconference - a live connection between people in separate locations for the purpose of communication.

Videoconferencing - communication across long distances with video and audio contact that may also include graphics and data exchange.

\section{6. List of Acronyms}

ACER Australian Council for Educational Research

ADB Asian Development Bank

ANU Australian National University

AusAID Australian Agency for International Development

CHEA Council for Higher Education Accreditation

CIS Commonwealth of Independent States

DLC Distance Learning Centre

EAP East Asian and Pacific (region or countries)

EBRD European Bank of Reconstruction and Development

ECA Europe and Central Asia

GDLN Global Development Learning Network

HEC Human Ethics Committee 


$\begin{array}{ll}\text { IP } & \text { Internet protocol } \\ \text { ISDN } & \text { Integrated Services Digital Network } \\ \text { JBIC } & \text { Japan Bank for International Cooperation } \\ \text { JICA } & \text { Japan International Cooperation Agency } \\ \text { LAC } & \text { Latin America and the Caribbean } \\ \text { MENA } & \text { Middle East and North Africa } \\ \text { MUH } & \text { Modern University for the Humanities } \\ \text { NGO } & \text { Non-governmental organisations } \\ \text { NZAID } & \text { New Zealand Agency for International Development } \\ \text { PLVF } & \text { Pacific Leaders' Virtual Forum } \\ \text { SIT } & \text { Social Identity theory } \\ \text { UN } & \text { The United Nations } \\ \text { UNDP } & \text { United Nations Development Programme } \\ \text { VLE } & \text { Virtual Learning Environment } \\ \text { VUW } & \text { Victoria University of Wellington } \\ \text { WB } & \text { The World Bank } \\ \text { WBI } & \text { World Bank Institute }\end{array}$




\section{Chapter 2: Literature review}

\section{1. Introduction}

This thesis is located at the intersection of three subject domains: Information Systems, Intercultural Communication and Organisational Behaviour. Therefore, this chapter provides a review of current literature related to the key notions of the research questions: intercultural and cross-cultural communication as well as development communication, videoconferencing and its perceived effectiveness.

Figure 2 Research domain

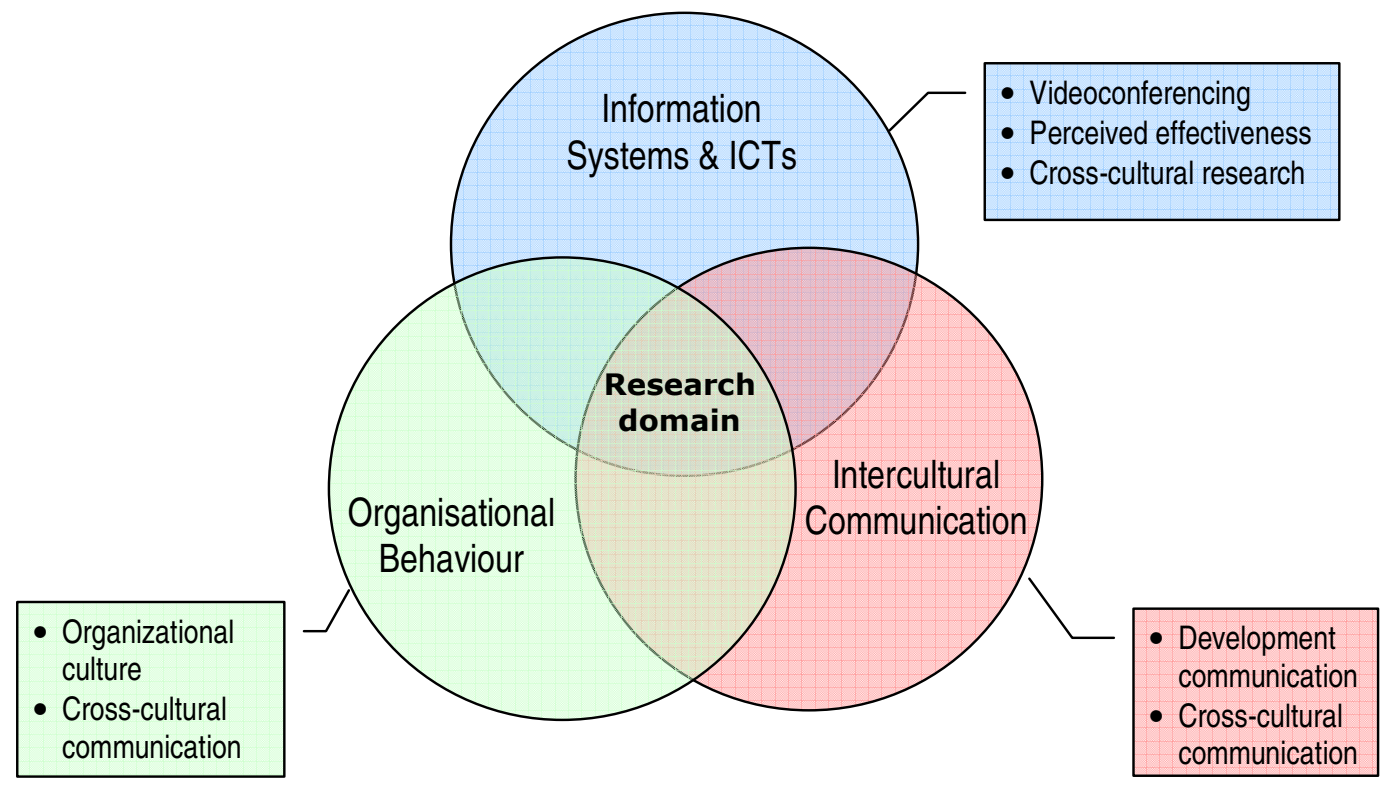

The research reported on this thesis looked into the process of intercultural communication that occurs at individual, organisational and national levels. Therefore, the field of intercultural communication and its integral parts, crosscultural and development communication are introduced in Section 2.2. This section also includes a review on the concept of culture and its influence on human behaviour and communication. The concept of organisational culture is also covered in this section because it has an important influence on the communication under this study. 
A review of media choice theories is presented in Section 2.3 to explain to the readers how people select media channels, such as videoconferencing, when communicating with others, especially when communicating across cultures and other boundaries.

Section 2.4 presents the videoconferencing technology and its use in distance learning, organisational communication and development communication. Theories that are often cited in IS research on video-mediated communication are briefly reviewed in this section. The benefits and challenges of videoconferencing technology are discussed from educational, organisational and development communication perspectives.

The effectiveness of video as a channel for communication is addressed in Section 2.4 with focus on intercultural interaction. The review is presented in an order consistent with the previous section: the effectiveness of videoconferencing for distance learning, organisational communication and development communication purposes.

The review of current cross-cultural research in IS in Section 2.5 reflects on key cultural issues, frequently applied methodologies, existing as well as newly emerging models and challenges in cross-cultural research domain.

Cross-culturally framed information systems research is reviewed in order to demonstrate the many ways in which cultural differences influence the development and use of information and communication technologies.

Selected theories that informed my research are reviewed in Section 2.7 including the model of national culture. This was done to indicate my initial consideration of this model as a candidate theory base for my study and explain the preference of an alternative approach, the social identity theory.

Finally, Section 2.8 addresses the gaps in research and justifies the appropriateness of the research questions. A summary of literature review concludes the chapter. 


\section{2. Intercultural communication}

The emergence of intercultural communication scholarship is linked with a particular historical episode in post World War II America when the United States' position as a new world power was on the rise (Shome \& Hegde, 2002). Intercultural communication emerged from occurrences at the Foreign Service Institute of the U.S. Department of State between 1946 and 1956 (LeedsHurwitz, 1990, p. 262): “Typically, Edward Hall's book, The Silent Language, published in 1959, is listed as the first work in the field, and often specifically mentioned as the crucial starting point".

The roots of what is now known as the field of intercultural communication were established when Foreign Service Institute hired some of the best linguists and anthropologists of the day to teach American diplomats foreign language skills as well as knowledge on how to interact with individuals in the specific culture to which they were being sent (Leeds-Hurwitz, 1990). President Kennedy's creation of the Peace Corps in the early 1960s increased interest in knowing more about how people of diverse cultures could communicate more effectively (Jandt, 2001).

With its primary focus on direct encounters of individuals of differing cultural and sub-cultural backgrounds, intercultural communication is generally defined in terms of two central concepts, culture and communication (Kim, 2005). Establishing this fundamental link between culture and communication helped the field of intercultural communication define its unique research agenda: to document the cultural variations in communication patterns (Zaharna, 2000). The relationship between culture and communication is seen as more reciprocal than causal, where culture may influence communication but is also constructed and enacted through communication (Martin \& Nakayama, 1999).

\section{Culture's influence on communication}

Communication theories are based on the idea that communication is purposeful. There is a sender, a receiver, content to be communicated, a medium for transmitting the information, and a social and cultural context. 
Communication involves the exchange of meaning between individuals, it also includes individual's behaviour which is perceived and interpreted by others (Adler, 2002). To understand how people from different cultures communicate with each other, it is necessary to understand how culture influences the way we behave. Bennett and Bennett (2001) believe that behaviour does not exist separately from thought and emotion although the primary emphasis of intercultural communication is on behaviour.

"Our culture influences our behaviour directly through the norms and rules we use to guide our behaviour when we interact with others. Our culture also indirectly affects our communication through the individual characteristics we learn when we are socialised into our culture" (Gudykunst \& Kim, 1997, page 18). Our culture provides us with a system of knowledge that generally allows us to know how to communicate with other people and how to interpret their behaviour (Keesing, 1974).

The cultural orientation of a society reflects the complex interaction of values, attitudes and behaviours displayed by its members (Hofstede, 1998).

"As shown in Figure [3], individuals express culture and its normative qualities through the values they hold about life and world around them. These values in turn affect their attitudes about the form of behaviour considered most appropriate and effective in any given situation. The continually changing patterns of individual and group behaviour eventually influence the society's culture, and the cycle begins again" (Adler, 2002', page 17). 
Figure 3 Influence of Culture on Behaviour and Behaviour on Culture

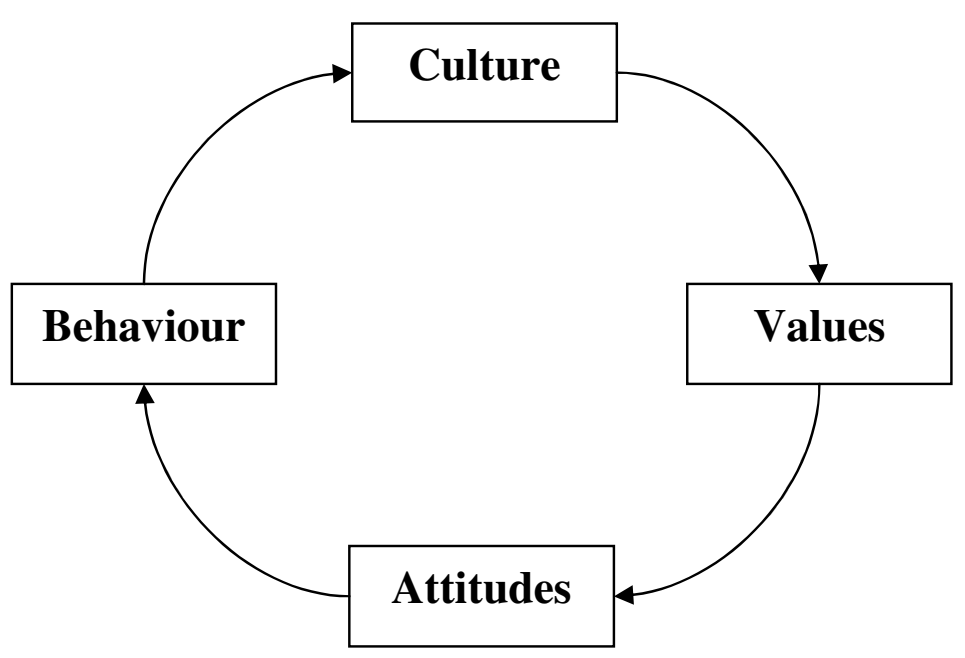

Perspectives of culture in intercultural communication

Researchers in intercultural communication generally discuss culture from two perspectives: objective and subjective. Objective culture refers to the products of culture such as art, literature, painting, and music, but also political, economic, social and linguistic systems, and institutions such as family, marriage and religion (Berger \& Luckmann, 1972).

In contrast, subjective culture is the learned and shared patterns of beliefs, assumptions and values of groups of interacting people that are displayed through their behaviours:

"Subjective culture refers to the psychological features that define a group of people- their everyday thinking and behaviour-rather than to the institutions they have created. Understanding subjective culturesone's own and others'-is more likely to lead to intercultural competence." (Bennett, 1998, p. 3)

Social reality is constructed of both objective and subjective aspects of culture; people learn how to behave through socialization into the institutions of the culture, which leads them to behave in ways that perpetuate those same institutions (Berger \& Luckmann, 1966). My research focuses on subjective rather than objective culture. 
Samovar et al. (Samovar, Porter, \& Jain, 1981, p. 24) suggested that one of the primary functions of culture is to define norms for interpersonal communication:

"Culture and communication are inseparable because culture not only dictates who talks with whom, about what, and how the communication proceeds, it also helps to determine how people encode messages, the meanings they have for messages, and the conditions and circumstances under which various messages may or may not be sent, noticed, or interpreted. In fact, our entire repertory of communicative behaviours is dependent largely on the culture in which we have been raised. Culture, consequently, is the foundation of communication. And, when cultures vary, communication practices also vary".

\section{Intercultural mindset and skill set}

The necessary unity of behaviour, thought and emotion can be called the intercultural mindset and skill set (Bennett \& Bennett, 2001, pp. 6-7):

- The mindset refers to one's awareness of operating in a cultural context. This usually entails some conscious knowledge of one's own culture (cultural self-awareness), some frameworks for creating useful cultural contrasts (e.g., communication styles, cultural values), and a clear understanding about how to use cultural generalizations without stereotyping. The mindset (or better, "heartset") also includes the maintenance of attitudes such as curiosity and tolerance of ambiguity that act as motivators for seeking out cultural differences.

- The intercultural skill set includes the ability to analyze interaction, predict misunderstanding, and fashion adaptive behaviour. The skill set can be thought of as the expanded repertoire of behaviour-a repertoire that includes behaviour appropriate to one's own culture, but which does not thereby exclude alternative behaviour that might be more appropriate in another culture. 
The importance of developing a global mindset that combines openness to and awareness of cultural differences between countries and bridges this gap has been amply discussed by scholars in the fields of organisational communication and international business (Gupta \& Govindarajan, 2002; Nardon \& Steers, 2008).

Understanding of the concepts of mindset and skill set informed my study and helped me in interpreting the perceptions, thoughts and behaviours of research participants.

\section{Concept of culture in organisational setting}

A large number of definitions have been proposed for the concept of culture in the corporate setting. These definitions outline the common key elements (Wilson, 2001):

- organisational culture is a shared phenomenon; it is a learned product of group experience and is, therefore, only to be found where there is a definable group with a significant history;

- there are two levels of culture: the visible level (behaviour patterns, the physical and social environment and the written and spoken language used by the group) and the deeper, less visible level (group's values (groupspecific and acceptable norms, values and behaviour);

- the manner in which new members learn the culture: informally from the existing employees and formally through induction training programmes;

- organisational cultures tend to change slowly over time. For example, the culture of the World Bank evolved as a result of change in leadership: from "lending culture" during McNamara's presidency in 1968-1987 (Milobsky \& Galambos, 1995) to a "results culture", initiated by Jim Wolfensohn when he entered office as president in June 1995 (Bare, 1998). This dramatic shift in organisational culture was a result of a US\$250 million, thirty-month reform initiative named the Strategic Compact, aimed at re-establishing the World Bank's pre-eminent position as the world's leading development agency (Weaver \& Leiteritz, 2005). 
Hofstede and colleagues (Hofstede, Neuijen, Ohayv, \& Sanders, 1990) suggest that there are three layers of culture that can be identified in an organisation. The initial layer consists of national culture, then occupational culture and the top layer consists of characteristics unique to the organisation, the corporate culture. Occupational culture has been defined as common values and worldview resulting from sharing the same occupation, across national differences (Hofstede, Neuijen, Ohayv, \& Sanders, 1990).

Culture and the communication norms within the organization is one of factors that influence adoption of videoconferencing technology because it will largely depend on how specific user groups interact with, change, or adopt the technology within their own unique workplace environments (Campbell, 2006). Companies can have multiple cultures (Kotter \& Heskett, 1992) or subcultures (Wilson, 1997) usually associated with different functional or geographic groupings (Wilson, 2001). Based on my personal experience, staff of the Operations Policy department of the World Bank that deals with lending operations shares a certain culture that is quite different from that in the Information Solutions Group where all the IT experts are. In parallel to these sub-cultures, staff of the World Bank Resident Representative office in Ulaanbaatar would share a unique culture, distinct from that in other country offices, for example the Tokyo office of the World Bank in Japan although they are both located in the Asian region.

The primary interest of my study was focused on individuals that belong to a social group characterised by common interest on development agenda. These individuals were men and women of different ages and nationalities (locals and expatriates), working for various organisations (government agencies, international organisations, NGOs, academic institutions, private sector) and playing different roles in those organisations ranging from high-level government officials to civil society leaders, professors and students.

\section{Dimensions of intercultural communication}

The diverse conceptualisations of intercultural communication were integrated by Kim (1984) who identified three organising dimensions: 1) the level of 
cultural group membership of communicators; 2) the social context in which intercultural communication takes place; and 3) the communication channel through which intercultural communication occurs.

Figure 4 Levels of cultural group membership of communicators (Y. Kim, 1984)

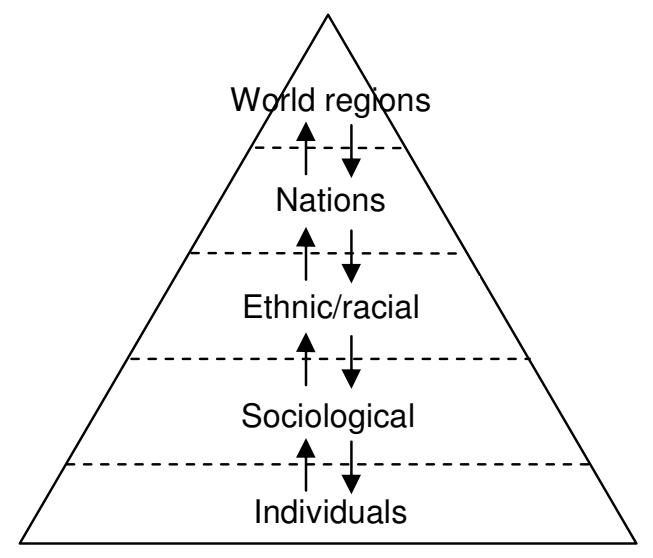

Figure [4] shows the common referents of the term 'culture' that generally include: world regions (such as Eastern culture and Western culture), world sub-regions (such as Latin American culture and South-East Asian culture), nations (such as Russian culture or Chinese culture), ethnic-racial groups within a nation (such as Black American or Maori culture), and various sociological subgroups categorised by sex, social class, geographic regions, and countercultural groups (such as Hippie culture, prison culture, street culture), among others (Y. Kim, 1984).

The specific social context in which an intercultural encounter takes place provides the intercultural communicators with specific role relationships, expectations, and behavioural norms and rules (Y. Kim, 1984). Figure [5] provides some examples of social contexts that are common in intercultural communication. 


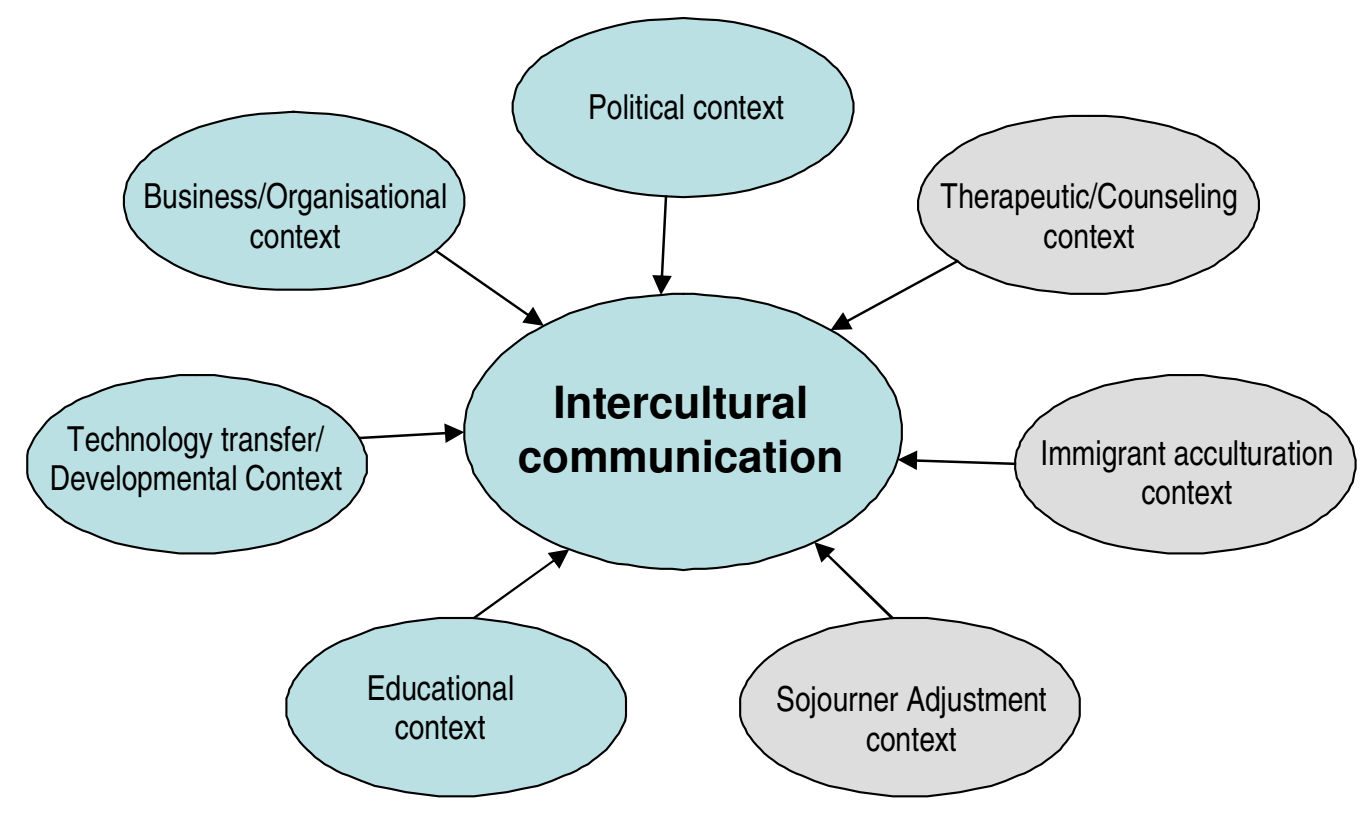

The contextual background of the intercultural communication under this study is exchange of knowledge in the area of development. It falls under Technology transfer/Developmental and Educational contexts of the above figure where 'technology transfer' emphasises the gap between the assumptions inscribed in the technologies developed in the context of industrialized countries and the prevailing way and state of organizational life in the countries to which the technologies were being transferred (Sahay \& Avgerou, 2002). The assumption was made that the specific organisational and political contexts also influence the intercultural communication under this study.

Another dimension by which intercultural communication can be further explored is the channel through which it occurs (Y. Y. Kim, 1984). Rapid development of information and communication technologies provided new media for long distance communication introducing computer-mediated communication, telegraphy and computer networks (digital telecommunications) in addition to traditional telephony, radio, and TV broadcasts (analog telecommunications). Four communication channels commonly referred to in IS research include face-to-face, text, audio and video 
(Chidambaram \& Jones, 1993; Keil \& Johnson, 2002; Rice, 1993; Suh, 1999; Wilson, Morrison, \& Napier, 1997/1998).

The intercultural communication under this study occurs through the video channel. In my inquiry, I observed actual videoconferences and recordings, interviewed videoconference participants and people who participated in other videoconferences. It must be noted that GDLN events take place as a result of a thorough preparatory work that involves prior communication between the parties mainly via e-mail and sometimes by telephone. Therefore, it is possible that respondent's perception about the effectiveness of communication during a GDLN event might have been influenced by the earlier communication on the matter that occurred via channels, other than video.

\section{Scholarship}

In analysing the development of intercultural communication as a research field, Zaharna (2000) noted that:

"The primary research goal of intercultural communication was to increase understanding of culturally-mediated communication phenomena. However, an underlying goal stemmed from Hall's (1976) observation that much of a culture's influence was "out-of-awareness." Even though culture completely surrounded its own members, they lacked an "in-awareness" knowledge of its presence and force. Thus, the ultimate goal of intercultural scholars was to expose the hidden dimensions of culture that lay "out-of-awareness" and bring them "inawareness." (page 88)

Three research avenues emerge within intercultural communication: 1) culturespecific that focuses on identifying the communication behaviours of a specific culture; 2) culture-general that seeks to identify commonalities or universals across cultures; and 3) inter-cultural interaction that explores how different behaviours affect the communication process (Zaharna, 2000). Based on this classification, I conclude that my inquiry was relevant to all three areas of intercultural communication research because it focused on the process of communication when individuals from different cultures interact, looked into 
communication behaviours in specific cultures (Mongolia, Russia, New Zealand and Australia) and sought patterns across these cultures. My study was relatively specific because this intercultural interaction was mediated by videoconferencing technology and the context of communication under this study was related to development agenda.

\section{Cross-cultural communication}

In addition to studies addressing issues of intercultural encounters, a significant amount of work among intercultural researchers has been directed toward 'cultural (or intra-cultural) communication' and 'cross-cultural communication' (Kim, 2005). The difference between 'cultural' and 'cross-cultural' studies is distinguished as follows:

"Studies of cultural communication have focused on essential patterns of communication norms and practices in specific cultures and subcultures. Studies of cross-cultural communication, on the other hand, have compared communication-related phenomena in two or more cultural or sub-cultural groups, closely following the research tradition of crosscultural psychology. Today, both cultural communication and crosscultural communication are complementary and integral parts of the intercultural communications domain" (Kim, 2005`, page 556)

While distinction exists between the terms 'intercultural' and 'cross-cultural', these have frequently been used synonymously in the literature (Limaye \& Victor, 1991). A distinction between 'cross-cultural' and 'intercultural' communication is drawn by Gudykunst (2002) as: "Cross-cultural involves comparisons of communication across cultures; Intercultural communication involves communication between people from different countries" (page 19).

This research aimed to discover patterns in perceptions of participants for the effectiveness of communication when they engage in video-mediated knowledge sharing activity. In other words, this study sought similarities and differences in perceptions of people from different cultural backgrounds by comparing the findings obtained through observations and interviews. The 
interviews with participants revealed their perceptions at various levels: individual, organisational and national. By analysing this data, I looked at intercultural as well as cross-cultural communication phenomena because this study compared subcultures across four different cultures: for example, the extent to which DLC staff (facilitators) in all four countries share common goals and guidelines; whether the participants in Australia and New Zealand are guided by similar intentions; and how different or similar are the expectations of participants in Mongolia and Russia.

\section{Development communication}

\section{Concept and definition}

World War II was the breaking point that completely changed the relationships between the continents and between the rich and poor countries: nearly all former colonies became independent states, freedom was recognised as a fundamental human right and many programs of development aid started implementation (Hofstede, 2001, p. 437): "The colonial jargon faded out: 'underdeveloped areas' were renamed 'developing countries' or the 'Third World', and development aid became 'assistance' and after that 'development cooperation'"'.

Whereas the main focus of intercultural communication studies has been the direct contact and interaction of individuals of differing cultural backgrounds, development communication studies have been aimed primarily at explaining the role of mass media in bringing about social change and economic development in Third World countries (Kim, 2005). The field was reconceptualised by affirming the intentional use of communication technologies and processes to advance socially beneficial goals, focusing on the use of communication for development (Wilkins \& Mody, 2001).

In some literature (Jacobson \& Storey, 2004; Steeves, 2001; Wilkins \& Waters, 2000; Wilkins, 1999; Wilkins \& Mody, 2001), development communication is understood within the context of institutional discourse that represents the interpretation of development institutions working in their global context, rather than interpretations of individual practitioners (Wilkins, 1999). 
Development communication is a social process based on dialog using a broad range of tools and methods. It is also about seeking change at different levels, including listening, building trust, sharing knowledge and skills, building policies, debating, and learning for sustained and meaningful change (World Bank, Food and Agriculture Organization of the United Nations, \& Communication Initiative, 2006).

There are three perspectives or ways of thinking about and practicing development: the first, modernisation, assumes that the Western model of economic growth is applicable elsewhere and that the introduction of modern technologies is important in development; the second or critical perspective challenges the economic and cultural expansionism of modernisation and argues for political and economic restructuring to produce a more even distribution of rewards in society; and the third, liberation perspective prioritises personal and communal liberation from oppression, as the key to empowerment and self-reliance, which is the goal of development (Melkote \& Steeves, 2001).

"Development communication involves creating mechanisms to broaden public access to information on reforms; strengthening the ability of governments in developing countries to listen to their constituencies and negotiate with stakeholders; empowering grassroots organizations to achieve a more participatory process; and undertaking communications activities that are grounded in public opinion research" (The World Bank, 2007).

Initiated under the leadership of the World Bank, GDLN aims to practice a participatory approach in communicating development agenda.

\section{Development Communication and Communication Technology}

The correlation of development communication scholarship and practice with rapidly advancing communication technologies can be traced through three distinct periods identified by Singh (2002) as follows:

- The first period - from late 1950s to early 1970s - was dominated by ideas of modernisation assuming that broadcast oriented channels of mass media 
communication can help poor people to imagine alternative futures by replacing traditional way of thinking with modern mindset. Communication development projects considered that television and radio are particularly effective for bringing awareness about health, nutrition, farming, participation, etc. to illiterate population. This model of mass-media communication was later criticised for being conservative, westerndominated and top-down instrumentality and ignorance of local context.

- The period from early 1970s to early 1990s is marked by increased attention to technologies such as satellites and telecommunications. Although satellite had attractive features (access to remote areas, more effective broadcasting of voice and image streams simultaneously compared to radio and its potential for interactive two-way communication), cost was the main barrier for indebted developing country governments to own satellites. In spite of that, many poor countries made telecommunications a development priority: socioeconomic benefits of two-way telephony included diversifying the rural and urban economic bases, reducing business and administrative costs and delivering social services.

- In the third period (from 1990s to present), the focus shifted to newer communication technologies: computers and the internet. "The current, "internet era" is marked both by an optimism about group-specific participatory and strategic approaches and the radical /structuralist pessimism regarding the potential of communication technology for development" (Singh, 2002', page 480).

A central concern with new technologies is that of access: access to the physical instruments (such as computers and telephone lines) and expertise (such as familiarity with keyboards and literacy in dominant languages) may be seen as a critical first step toward enabling the potential of computer technologies (Wilkins \& Waters, 2000). For example, the lack of access to the Internet for scientists in the developing world is creating a new form of poverty information poverty - which is making it harder for them to stay abreast, let alone catch up with their colleagues in the developed world (Arunachalam, 1998; Britz, 2007). If at all possible, access to information, like other services, 
should not be commodified, but could be conceived as a human right (Wilkins, 2002)

There had been some debate as to whether information and communication technologies (ICTs) were relevant to developing countries, but this debate has been resolved with a clear "yes" answer; the question has become not whether, but how ICTs can be beneficial (Walsham, 2006).

\section{Dominant Models in Development Communication}

The field of development communication is dominated by two conceptual models: diffusion and participation (Morris, 2003):

The diffusion model, named for Roger's (1962) diffusion of innovations theory, regards behaviour change as the goal of a communication campaign and views persuading individuals to change their ideas by providing them new ideas and information. Activities such as entertainment-education and social marketing fall within the diffusion model. Entertainment-education is the process of purposely designing media messages and broadcasting via radio and television in order to increase audience knowledge about an educational issue, create favourable attitudes and change overt behaviour (Melkote \& Steeves, 2001). Social marketing is defined as "the adaptation of commercial marketing technologies to programs designed to influence the voluntary behaviour of target audiences to improve their personal welfare and that of the society of which they are a part"'(Andreasen, 1994', page 110)

The participatory model posits that development communication is not a vertical process of information transmission from the knowledgeable to the less knowledgeable, but rather a horizontal process of information exchange and interaction (Morris, 2003). The participatory model stresses the importance of cultural identity of local communities and of democratisation and participation at all levels - international, national, local and individual (Servaes \& Malikhao, 2004). Therefore, for participatory theorists and practitioners, development communication required sensitivity to cultural diversity and specific context that were ignored by modernization and diffusion theories (Waisbord, 2001). 
Although the two approaches differ in the underlying philosophies, frameworks, program strategies, and measurement tools, the advocates of both participatory and diffusion models should borrow useful elements one from another for more effective communication (Morris, 2003).

Depending on the context in which the program is implemented, the priorities of funding agencies, and the needs of the communities, development communication practitioners employ appropriate techniques from the 'tool-kit' of approaches that includes information dissemination and entertainmenteducation, behaviour change, social marketing, social mobilization, media advocacy, communication for social change, and participatory communication (Waisbord, 2001).

Table [1] explains the differences between the two approaches.

Table 1 The changing communication environment (Servaes \& Malikhao, 2004)

\begin{tabular}{|c|c|}
\hline Traditional & New \\
\hline $\begin{array}{l}\text { Vertical communication }- \text { from } \\
\text { government to people }\end{array}$ & $\begin{array}{l}\text { - Horizontal communication - } \\
\text { people to people }\end{array}$ \\
\hline - Unipolar communication systems & - Communication networks \\
\hline - Few information sources & - Many information sources \\
\hline $\begin{array}{l}\text { - Easy to control - for good (generating } \\
\text { accurate information to large } \\
\text { numbers of people) and ill } \\
\text { (government control and censorship) }\end{array}$ & $\begin{array}{l}\text { - Difficult to control for good } \\
\text { (more debate, increased voice, } \\
\text { increased trust) and ill (more } \\
\text { complex, issues of accuracy) }\end{array}$ \\
\hline - Send a message & - Ask a question \\
\hline
\end{tabular}

\section{Knowledge and knowledge transfer in development}

Conceptions of knowledge and learning are often taken for granted in accounts in development studies and mainstream development. According to Stiglitz (2004), globalization of knowledge is the free flow of ideas and information that has followed the lowering of communication costs and the closer integration of societies. The Knowledge for Development issue of the World Development Report series (1999) makes a distinction between two sorts of knowledge that are crucial for the developing world: knowledge about technology or knowhow, for example in health care or infrastructure financing; and knowledge 
about attributes such as the quality of a product or the credibility of a borrower. This approach has been criticised by McFarlane (2006) and other authors in post-development and anthropological scholarship (Escobar, 1995; Ferguson, 1994; Hobart, 1993; Moore, 1996) as the rationalist approach to knowledge in development that conceives of knowledge as objective and universal, as a technical entity that can be shifted in a linear way unchanged from the World Bank and North to South, and in so doing separates the conception of knowledge from politics and context. Knowledge at the World Bank has been hegemonic and homogenous rather than heterogeneous and plural but have often had to be accepted by developing countries due to the dependence on its financial power (Mehta, 1999).

A post-rationalist perspective that is not opposite, but different from the World Bank approach, emphasizes: the crucial role of practices in knowledge creation and learning; the importance of conceiving learning as a social process; the need to recognize spatial relation in knowledge creation rather than emphasizing an 'in-here' (local) 'out-there' (global) ontology of knowledge creation; the need to recognize the inherent material nature of knowledge creation; and most importantly the need to recognize that conceptions of knowledge and learning are often highly political, whether from the World Bank or a community based NGO (McFarlane, 2006).

Through its extensive network, GDLN facilitates participatory communication for development by offering knowledge sharing activities and promoting dialogue between government officials, civil society activists, private sector, development practitioners and experts around the world. Whether offering knowledge sharing and educational activities - from health, education or rural development to private sector development, financial reform or judicial reform - the aim is to build consensus through raising public understanding and generating well-informed dialogue among stakeholders (The World Bank, 2007). 


\section{Avenues for future research}

The role of new technologies in strategic social change is identified as an important area for future research in development communications scholarship: for instance, a) the ability of these new technologies to facilitate change in the society; b) the degree to which they are employed to facilitate the creation of mediated content on development, and not just distribution of existing information; c) the nature of discussion and practice within development networks, such as GDLN; or d) the social location of those who participate in an interaction, considering characteristics such as age, gender, race, and socioeconomic status, the relative heterogeneity of interaction participants, and the social distance between them (Wilkins, 2002).

Scholars are urged to systematically examine the impact of technology on the very nature of social interaction and devote more serious attention to the following five key dimensions (Cerulo, 1997):

- The context in which the communication occurs and the number of individuals involved in sending and receiving the message.

- The form of the communication, distinguishing direct from mediated interaction, and one-way versus two-way exchange.

- The communication content, referencing instrumental versus expressive content, impersonal versus intimate content, and factual versus fictional material.

- The social location of those who participate in an interaction, considering characteristics such as age, gender, race, and socioeconomic status, the relative heterogeneity of interaction participants, and the social distance between them.

- The character of the exchange, noting the nature of the benefits that emerge from the exchange (e.g., bilateral versus unilateral).

Also, there is a need for greater sophistication in understanding the complexities of knowledge and learning and the relationship between travel, knowledge and place in development, because the ways in which these 
development rubrics are conceived has consequences for development practices (McFarlane, 2006).

I believe that the focus on a particular communication channel (video), choice of a specific context of global development learning network and attempt to understand the influence of cultural, sub-cultural and inter-group differences on development communication made the current research worth exploring.

\section{3. Media Choice Theories}

Media choice theories attempt to explain why individuals use certain channels in certain situations and what the outcomes are of such use (Karahanna, 1995; Ngwenyama \& Lee, 1997). Numerous theoretical models predict that media selection is a function of factors such as media and message characteristics (Daft, Lengel, \& Trevino, 1987). Two similar approaches that explain how users come to ascribe certain characteristics to the media and tasks that influence media selection were developed independently by two groups: Media Richness theory by organisation scientists in the US and Social Presence theory by business communication scientists in Great Britain (Carlson \& Davis, 1998).

In IS research, Media Richness theory (Daft \& Lengel, 1984) is frequently cited when video is compared to other communication media in terms of communication effectiveness (Carlson \& Davis, 1998; Dennis \& Kinney, 1998; Panteli \& Dawson, 2001; Suh, 1999). Computer-mediated and video-mediated communication have been retroactively fit into the theory's framework that has been initially developed to compare face-to-face communication to telephone and printed text (El-Shinnawy \& Markus, 1997).

Face-to-face communication is perceived as the highest medium from media richness perspective, followed by video, audio and text (Dennis \& Kinney, 1998; Suh, 1999). 'Rich' channels, such as face-to-face and videoconferencing, enable rapid feedback, provide multiple cues via body language and tone of voice and message content is expressed in natural language (Ngwenyama \& Lee, 1997) whereas 'lean' channels such as e-mail are more useful for reducing uncertainty (Daft \& Lengel, 1986). 
However, this point of view has come under increasing scrutiny as some argue that media richness is a subjective rather than objective characteristic (Fulk, Schmitz, \& Steinfield, 1990) or that other media qualities such as synchronicity exert influence on media choice (Dennis, Valacich, Speier, \& Morris, 1998). Some researchers have simply concluded that appropriate media choice is more a function of preference, convenience and cost than of task media fit (Johansen, Vallee, \& Spangler, 1979). Despite a great deal of research on media richness, clarity on the issue of media effectiveness remains a challenge (Leonard, VanScotter, \& Pakdil, 2009).

Various media is also compared in light of Social Presence theory where "social presence is the degree to which the medium facilitates awareness of the other person and interpersonal relationships during the interaction" (Fulk, Schmitz, \& Steinfield, 1990', page 118). Being aware of the social presence of a medium may be important to understanding 'person-oriented' or social tasks (Dennis \& Kinney, 1998). Whereas task-oriented activities such as problem solving might be carried out equally well using any medium, person-oriented activities such as conflict resolution are thought to require media high in social presence (Miranda \& Saunders, 2003). Electronic and printed communication media are typically viewed as low in social presence while face-to-face communication is viewed as high in social presence. A study on the use of videoconferencing in light of social presence suggested that increases the learner's ability in social and emotional expression, thus improving communication which may enhance learning satisfaction (Giesbers, Rienties, Gijselaers, Segers, \& Tempelaar, 2009).

\subsection{Videoconferencing}

Simply described, videoconferencing is a two-way or multi-way phone call with moving pictures, which can be used person to person or group to group at two (point to point) or more (multi-point) locations. Typically, the connections are made over one of the three options: by ISDN, satellite, or IP. A point-topoint connection can be made directly between two points. If there are more than two points, or sites, as they are sometimes called, in a videoconference, then a "bridge" or multipoint control unit (MCU) must be used. An MCU is like 
a telephone exchange to where all calls are routed and then patched together to form one 'conference' (Global Development Learning Network, 2005). Important is the fact that videoconferencing occurs synchronously, enabling participants to communicate interactively in real time.

Most people associate the use of videoconferencing with the use of computers, but in actuality, the first prototypes were developed long before computers and internet: the video signal for the PicturePhone, introduced in 1964 at the New York World's Fair, was transmitted over ordinary analog telephone lines (Bekkering \& Shim, 2006). Today, advanced IP-based videoconferencing technologies afford quality of interaction significantly better than those documented in prior studies (Wolfe, 2007) and this continues to improve rapidly.

Videoconferencing technology is widely used not only in distance learning and organisational communication as discussed later in the chapter, but is successfully applied in telemedicine (Marziali, Donahue, \& Crossin, 2005; Rees \& Stone, 2005; Simpson, Bell, Britton, \& Mitchell, 2006); legal system (Lexcen, Hawk, Herrick, \& Blank, 2006; Price \& Sapci, 2007; Treadway Johnson \& Wiggins, 2006; Wiggins, 2006), immigration services (Federman, 2006); even a choreography can be created in a video-conferencing environment (Naugle, 2002).

A videoconference is highly appropriate for structured events (Panteli \& Dawson, 2001) with formal agenda and handovers of the floor to participants for presentations, comments or questions, and answers. To facilitate efficient use of connection time, GDLN events are well prepared for and have predefined running order. Strategies which make for successful videoconferencing can be shared in a simple few short training sessions (Arnold, Cayley, \& Griffith, 2002).

Much of the development and use of videoconferencing systems has been driven by technology advances with little consideration for human communication therefore the developers need to understand the nature of the 
communication that takes place in a particular location or context (Patrick, 1999).

Issues related to cross-cultural interaction and communication were recommended as worth further research to better understand what barriers learners do encounter and how they do or do not manage related incompatibilities (Martin, 2005). This proposed study adequately fits into this research domain.

The rapid development and diffusion of information technology has led to new ways of learning and scientific research, allowing researchers to engage in more complex and data-intensive areas of research, and has also changed the innovation process, e.g. in allowing greater international co-operation and networking (OECD, 2006).

\section{Videoconferencing in Distance Learning}

In the literature, the term 'distance learning' is used in relation to a wide variety of topics including but not limited to education, organisational communication, information systems, knowledge management, telemedicine, governance, management, marketing, business administration and development communication.

The term 'distance learning' has many synonyms that often hide real differences in types of learning and forms of delivery and is growing wider in scope as it embraces new forms of provision delivered by a range of providers alone or in collaboration (Middlehurst \& Woodfield, 2006). The terms 'distance education' or 'distance learning' have been applied interchangeably by many different researchers to a great variety of programs, providers, audiences, and media (Sherry, 1996). Distance learning usually implies some sort of technological support through the internet, email and videoconferencing (Martz, 2005). Distance education research includes a body of work specifically on the use of videoconferencing in education. This research follows the vast growth of national and international videoconferencing networks and an

increasing number of practitioners keen to understand the value of this technology, yields a number of important conclusions (Allen, Sargeant, Mann, 
Fleming, \& Premi, 2003; Badenhorst \& Axmann, 2002; Bernard et al., 2004; Ferry, Kydd, \& Boyles, 2012; Greenberg, 2004; Peterson, 2004):

- For delivering instruction, videoconferencing is likely to be neither more nor less effective than its counterpart, the "traditional" classroom².

- Students and teachers alike, find videoconferencing as satisfactory as traditional classroom learning formats.

- Interactivity is the unique capability of videoconferencing technology, it supports far greater interaction than many asynchronous technologies. Effective videoconferencing-based instruction must be designed to take advantage of this capability.

- Videoconferencing for teaching requires additional skills to those of a classroom educator wherefore without adequate training of the teaching staff and their learners, systems would be underutilised

- When used appropriately, the benefits of videoconferencing justify the upfront cost of adopting the technology. It then becomes a cost-effective way for educational institutions to deliver successful learning experiences to an expanded student population.

More than one-third of public high schools in the US offer distance learning courses of which two-way interactive video is the primary instructional delivery mode (Setzer \& Lewis, 2006). Videoconferencing is successfully used for enhancement and improvement of teaching and learning in American high schools enabling the students to interact with experts and scientists as well a with students at others schools (Cole, Ray, \& Zanetis, 2004; McCombs, Ufnar, \& Shepherd, 2007; Merrick, 2005). Previously, the cost of maintaining dedicated telephone lines for ISDN videoconferencing connections has been a major obstacle for the adoption in schools: line fees of hundreds of dollars per month for a resource that may be used only once or twice a month (Merrick, 2005). Nowadays, emergence of increasingly reliable IP network connectivity have made this learning tool affordable and accessible: more schools purchasing

\footnotetext{
2 "Traditional" classroom is often used as a benchmark when comparing face-to-face instruction to distance learning modes through other communication channels including video. It has to be noted that learning in "traditional" classroom may not necessarily be very effective.
} 
videoconferencing equipment and benefiting from the extensive pool of videoconference courses on various topics (Keystone Conference, 2005).

Colleges and universities around the world quickly perceived the potential of videoconferencing for reaching vastly expanded student populations at national and international level. Videoconferencing has seen rapid growth as an economical and effective distance learning technology in the United States (Setzer \& Lewis, 2006; Waits \& Lewis, 2004), Canada (Kanuka, Collett, \& Caswell, 2002; Wolfe, 2007), United Kingdom (Burns, 2002; Panteli \& Dawson, 2001), Ireland (Carville \& Mitchell, 2000), Australia (Campbell, 2006; Peszynski \& Yoong, 2002) and New Zealand (Hauber, Regenbrecht, Hills, Cockburn, \& Billinghurst, 2005; Waiti, 2005). Use of video channel is common in other parts of the world, for example in Russia (Bonham, Surin, Nakano, \& Seifert, 2003), United Arab Emirates (Selim, 2005), Thailand (Pagram \& Pagram, 2006), Turkey (Yazici, Altas, \& Demiray, 2001), Syria (Albirini, 2006), China (Gao, Lui, \& Mahanti, 2006; Rutkowski, Vogel, Bemelmans, \& van Genuchten, 2002).

Cross-cultural videoconferencing provides a qualitatively different experience compared to other forms of learning: the students are contributing to a truly international learning environment, where they can obtain information and knowledge, debate competing perspectives, and create products with colleagues with whom they would otherwise not have the opportunity to collaborate (Bonham, Surin, Nakano, \& Seifert, 2003).

After reviewing over two hundred items of distance education literature that compare distance learning methods with traditional classroom instruction, Bernard et al. (2004) encourage the key stakeholders to make sure that excellence and effectiveness of distance learning take precedence over cost efficiency.

The issue of quality appears throughout the distance learning literature where concerns have been expressed by both supporters and challengers of distance learning (Bower, 2001). Examples include investigation on the quality of teaching and learning via videoconferencing (Knipe \& Lee, 2002), a study on quality assurance for distance learning (Clarke, Butler, Schmidt-Hansen, \& 
Somerville, 2004) and a quality review of distance learning on a sample of five countries (Middlehurst \& Woodfield, 2006).

\section{Videoconferencing in Organisational Communication}

In this globalising world, business professionals increasingly using advanced communication technologies to collaborate across multiple cultures, locations and time zones. The business communication environment has transformed because more than 80 percent of the workforce work across locations (Richman, Noble, \& Johnson, 2002), crossing various boundaries, so that managers increasingly lead people, manage projects, and engage stakeholders in globally distributed environments (Starke-Meyerring, 2006). The key objectives behind the installation of videoconferencing systems in organisations are the need to enhance decision-making processes within meetings and to increase the speed with which meetings are held without reducing decision making quality (Campbell, 1997).

Studies on organizational uses of videoconferencing discovered that video meetings are efficient in that the communication is task-oriented, therefore a diverse array of organizational communication tasks can be successfully conducted via video conferencing (Fulk \& Dutton, 1984). However, some tasks such as bargaining, persuasion, coalition formation and meeting new people are better performed face-to-face whereas tasks such as routine information sharing, cooperative problem solving or brainstorming among people who know each other well (Mosier \& Tammaro, 1994) or communicating the progress of an ongoing project with well established relationships (Panteli \& Dawson, 2001) can be successfully performed via videoconferencing. Interestingly enough, the fewer cues are presented, the greater the psychological distance between the participants, and thus the more taskoriented and less spontaneous the style of communication during videoconferences (Sellen, 1995).

Even well established teams prefer video over audio because it enables greater mutual understanding among participants, makes video-mediated communication more efficient, effortless and improves the quality of 
collaborative work (Tang \& Isaacs, 1992). Users indicated a positive relationship between videoconferencing usage and personal standing in the organisation because it increases one's personal visibility within the organisation and enables greater access to key personnel (Campbell, 2006)

In order to successfully implement videoconferencing systems, organisations should provide training and documentation on the technology, clearly communicate strong management support for this system and explain the advantages of video over existing channels, provide opportunities for employees to gain experience and higher efficacy in using this system, and ensure that employees understand any privacy controls built into the system (Webster, 1998). The training programs should focus on 'winning the hearts and minds' of potential users, rather than on technology handling because an interest and willingness of employees to use and experiment with the technology is critical in effective use of videoconferencing system in the long run (Panteli \& Dawson, 2001). Strategies that boost self-confidence and reduce user apprehension and anxiety about using the technology should also be included in these training programs so that employees overcome personal anxiety and improve the effectiveness of video-mediated communication (Campbell, 2006).

Managers willing to create effective virtual teams should consider the psychological profile and personality characteristics of the specific team members because in order to be successful in computer-mediated environment, participants must possess patience, persistence, perseverance along with certain degree of tolerance, flexibility and understanding (Warkentin, Sayeed, \& Hightower, 1997).

\section{Videoconferencing in Development Communication}

The role of ICTs is recognised not only as a medium of communication, but also as a development enabler, and as a tool for the achievement of the internationally-agreed development goals and objectives (World Summit on the Information Society, 2005). Besides opening up new possibilities and visions on how the information technologies can provide a platform for enhancing 
countries' social and economic development, it has proven to be an important driver for the delivery of e-government, e-learning and other services (Gray, Magpantay, Thompson, de Ridder, \& Southwood, 2006). For example, an ongoing, large-scale action research project within the health care sector called Health Information Systems Project that has been implemented in a number of developing countries including Mongolia, contributed to the important debate on how ICTs in general, and information systems research in particular, can make a positive difference in the developing countries (Braa, 2004). ICTs can be powerful tools for sharing information, but they often cannot solve the development problems caused by the underlying social, economic and political issues, nor can they change the existing power structures as the information available is not necessarily knowledge (Servaes, 2007). In order to become knowledge, the information has to make sense to the people who receive this information (Gerster \& Zimmermann, 2005).

Literature in information systems and development communication domain lacks empirical evidence of using videoconferencing for the purposes of communicating development agenda across cultures.

\section{5. Perceived effectiveness of Videoconferencing}

In the literature, perceived effectiveness of video channel is discussed from different perspectives:

- as a global information system (Kim \& Oh, 2000),

- as a knowledge management tool (Wolfe, 2007),

- as a technology for virtual team collaboration (Olson \& Olson, 2000; Warkentin, Sayeed, \& Hightower, 1997)

- as a medium for organisational communication (Bekkering \& Shim, 2006; Chidambaram \& Jones, 1993; O'Connail, 1993),

- as a medium for teaching and learning on national level (Allen, Sargeant, Mann, Fleming, \& Premi, 2003; Burns, 2002; Carville \& Mitchell, 2000; Choi \& Johnson, 2005; Guzley, Avanzino, \& Bor, 2001; Piccoli, Ahmad, \& Ives, 2001; Stonebraker \& Hazeltine, 2004; Wilson, Morrison, \& Napier, 1997/1998) and intercultural level (O'Dowd, 2007). 
Review of effectiveness studies above will help me to understand the effectiveness of communication that occurs via video channel.

People from the same cultural and linguistic group may find it difficult to communicate effectively. However, additional problems arise when interactions stretch across cultures because in order to communicate effectively in the intercultural environment, it is essential to be knowledgeable about the cultural factors that affect the situation (Chaney \& Martin, 2003). Such cross-cultural interactions increase in frequency given the internationalisation of business and social life, which has been enabled primarily by advances in information technology (Martinsons \& Davison, 2003).

Communication effectiveness is influenced by five factors: source, message, channel, receiver, and destination (McGuire, 1989). This directly corresponds to Lasswell's (1948) well-known description of communication as "who, says what, through which channel, to whom, and with what effect" and Shannon and Weaver's (1949) linear model in which communication is described as a transmission process from a transmitter to a receiver by means of codification and de-codification of the information itself where subjects are considered as passive recipients of messages. The only addition, which differentiates their description from Lasswell's is the question of 'noise' (distraction such as people entering and leaving the room during a session, breaking off to answer cells phones, etc.) the receiver encounters when decoding (cognitively processing) the message.

For instance, when people communicate using computer-mediated information technologies such as videoconferencing, the effectiveness and style of their communication is affected (Dustdar \& Hofstede, 1999). Limited bandwidth and network delays could cause the moving images and audio to lose coordination, so image quality and delay of the picture transmission might affect the effectiveness of communication in video condition (Bekkering \& Shim, 2006; Valacich, Mennecke, Wachter, \& Wheeler, 1994).

Selecting credible sources, choosing message strategies, and determining optimal settings or channels for delivery of the communication affects 
effectiveness of communication; moreover, each of these three factors is affected by culture (Kreuter \& McClure, 2004). Ineffective communication can occur for a variety of reasons when individuals engaged in communication come from different cultures: pronunciation, familiarity with the topic being discussed, fluency in the other person's language and understanding of the social context (Gass \& Varonis, 1984). Effective communication involves minimising misunderstanding: whether or not a specific instance of communication is effective or not depends on the degree to which the participants attach similar meanings to the messages exchanged (Gudykunst \& Kim, 1997).

Perception involves our awareness of what is taking place in the environment: perception of each individual is unique and is based on culture, ethnicity, sex, background experiences and needs (Gudykunst \& Kim, 1997). Perceptions are formed in various ways: where we are born and raised, the language we learn, the people and environment with which we live, and the psychological stimuli we encounter (Chaney \& Martin, 2003). Since we know only what we have personally perceived and cannot know for sure what someone else has perceived, intercultural communication involving different cultures becomes particularly difficult (Singer, 1998).

Existing studies on perceived effectiveness of videoconferencing can be classified by the purpose of communication this media was used for: educational, organisational, knowledge sharing, etc.

\section{In Distance Learning}

Studies addressing the effectiveness of videoconferencing technology or its use in distance education were conducted at undergraduate and graduate levels involving range of subject areas including early childhood education (Carville \& Mitchell, 2000), business (Burns, 2002; Coventry, 1996; Guzley, Avanzino, \& Bor, 2001; Neuhauser, 2002; Stödberg \& Orre, 2010), medical studies (Burke, Chaney, \& Kirsten, 2010; Moridani, 2007), arts and music (Badenhorst \& Axmann, 2002; Riley, 2009), engineering (Asgill \& Bellarmine, 2003) and continuing education (Allen, Bourhis, Burrell, \& Mabry, 2002). 
For example, the effectiveness of combining interactive digital videoconferencing with a Web-based discussion forum created a new learning environment, where students in Japan, Russia, and the United States collaborate with their colleagues abroad to address current policy issues (Bonham, Surin, Nakano, \& Seifert, 2003).

Effective videoconferencing requires that teachers adapt not only content but also technique to account for the distributed, highly interactive nature of the pedagogical situation (Greenberg, 2004) because studies on perceptions of distance education instructors revealed that they were concerned by the emotional distance, feedback and lack of flexibility in distance delivered programs (Kanuka, Collett, \& Caswell, 2002).

Context-based videos in online courses have the potential to enhance learners' retention and motivation: learners perceived that video-based instruction were more motivating in terms of attention and more memorable than the traditional text-based instruction (Choi \& Johnson, 2005). Learners benefit in improved grades and coursework quality as well as advantages of videoconferencing over traditional learning technologies constitute the student perceived usefulness of videoconferencing technology (Selim, 2005).

Comparison of asynchronous video streaming to synchronous videoconferencing in distance learning at tertiary level revealed that students preferred a mix of interactive sessions and asynchronous video streaming over delivery of content using the synchronous or asynchronous method alone (Moridani, 2007).

\section{In Organisational Communication}

Globalisation of business highlighted the need for multinational and transcultural organisations to utilise information technology to achieve efficiencies, coordination and communication (Karahanna, Evaristo, \& Srite, 2002). In fact, globalisation and information technology continuously reinforce each other: globalisation enhances the necessity of innovation in information technology since multinational companies need to coordinate their global operations through information systems, while the very improvement in information 
technology forces the firms to become more global in their business because the advanced technologies nurture capable competitors on a global scale (Kim \& Oh, 2000).

In the context of organisational communication, prior research has focused on the effectiveness of videoconferencing for different communication tasks, comparisons between different communication media, the attitude of users and their acceptance of technology, and impact of meeting processes and outcomes (Campbell, 1996; Daft, Lengel, \& Trevino, 1987; Straus, Miles, \& Levesque, 2001).

The choice of communication medium and decision support systems has a significant impact on group perceptions and performance of teams in disbursed settings: managers are urged to augment existing communication systems such as audio-conferencing and teleconferencing with electronic meeting systems for more effective communication (Chidambaram \& Jones, 1993). Diversity was reported as a major strength that contributes to the effectiveness of multicultural virtual teams (DeSanctis, Wright, \& Jiang, 2001).

In organisational context, the explicit goal of videoconferencing is often stated as one of simulating face-to-face meetings; the implicit assumptions is that the ability to see the moving picture of the person with whom one is engaging in conversation is a crucial part of the communication process (Sellen, 1995). Users of videoconferencing systems perceive added value, reporting that video capability facilitates effective interaction and mutual understanding (Tang \& Isaacs, 1992).

The concept of "swift trust" was put forth by Meyerson, Weick \& Kramer (1996) to describe how members of temporary virtual groups may be able to accomplish tasks without having developed relationships first. The "swift trust" enables members to take action, and this action will help the team maintain trust and deal with uncertainty, ambiguity, and vulnerability while working on complex interdependent tasks with strangers in a situation of high time pressure (Jarvenpaa, Knoll, \& Leidner, 1998). This concept is applicable to videoconferencing where time is strictly limited and the participants have never met before and usually, do not meet again as the exact same group of people. 


\section{In Development Communication}

Little scholarly research has been found that combines the subject of development communication and video-mediated communication across cultures. Indication with regard to the use of videoconferencing systems by multilateral and bilateral development agencies can be found on their respective websites but not much evidence of research on its effectiveness in the context of development communication can be found in the scholarly literature.

A few anecdotal evidences of GDLN network usage were found in the process of literature review: an assessment of the chances for success of the francophone videoconferencing centres (Yao, 2003); a multi-case study on distance learning policies and practices in five countries where one of sites was the Jordan DLC (Middlehurst \& Woodfield, 2006); an experimental observation of IP/multicastbased real time instructional system in western China (Gao, Lui, \& Mahanti, 2006) and a case study on the example of Cote D'Ivoire DLC (Assié-Lumumba, 2008). A study that explores the potentials for offering wider access to tertiary education mentions that by joining the GDLN, the University of Southern Queensland is aiming at increasing student recruitment numbers (Huijser, Bedford, \& Bull, 2008).

Future research on the effect of culture on media effectiveness could integrate other variables that are directly related to effectiveness, such as task demands, receiver characteristics, organizational and professional or occupational culture, technology acceptance, and individual preferences (Leonard, VanScotter, \& Pakdil, 2009).

\section{6. Cross-cultural research in IS}

There has been increasing interest in the IS literature in the impact of cultural differences on the development and use of information and communication technologies (Myers \& Tan, 2002). Although culture is a challenging variable to research, in part because of the multiple divergent definitions and measures of culture, a wide body of literature has emerged that sheds light on the relationship of IT and culture (Leidner \& Kayworth, 2006). Still, many practical and theoretical questions remain unanswered (Karahanna, Evaristo, \& Srite, 
2002). This can be partly explained by methodological and resource difficulties inherent in cross-cultural research, the long time horizon required to complete/conduct these types of studies as well as by the lack of unanimity about the underlying meaning and definition of the construct 'culture' (Straub, 2002).

Studies on information technology and culture - at the national level (e.g. crosscultural studies) and at the organisational level - address similar research questions but use different values as the means of representing culture (Leidner \& Kayworth, 2006).

Over 60 percent of empirical cross-cultural studies utilised one or more of Hofstede's dimensions (Leidner \& Kayworth, 2006). They focused predominantly on issues related to IS management and types of information systems, including videoconferencing, and were mainly country-specific or technology-specific rather than comparative (Ford, Connelly, \& Meister, 2003).

Yet, a number of cross-cultural research in IS involves comparison between two or more cultures, for example: a survey on perceptions of IS professionals towards the importance of IS objectives (Peterson, Kim, \& Kim, 2003), a survey on patterns in mobile internet usage (Lee, Choi, Kim, Hong, \& Tam, 2004), a study on online collaborative behaviours (Kim \& Bonk, 2002), a survey exploring major determinants of instructor's intentions to teach online courses (Alshare, Kwun, \& Grandon, 2006), a laboratory experiment on escalation of commitment behaviour in software projects (Keil, 2000), an investigation of how bad news about failing IS projects are reported in different cultures (Tan, 2003) and a survey on mobile commerce services (Jarvenpaa, 2003).

The guidelines for cross-cultural research initially developed by an international committee of scholars with primary focus on research on psychological and educational issues (Hambleton, 1994; van de Vijver \& Hambleton, 1996) cover four domains: context (describing basic principles of multilingual studies), development (recommended practices in developing multilingual instruments), administration (issues in instrument administrations), and documentation/score interpretation (related to 
interpretation and cross-cultural comparisons of scores). These guidelines can be easily generalised to IS research (Karahanna, Evaristo, \& Srite, 2002).

IS researchers are called to conduct more in-depth case studies and ethnographies of the relationship between IT and culture in many different parts of the world and in doing so, go beyond the simplistic models of national culture (Myers \& Tan, 2002).

Although national culture still exists, differences also exist in individual level cultural orientations within the overall national culture, therefore newer, individual-level culture measures are likely to be more useful in pinpointing cultural issues in IS research than usual "comparative" scores (McCoy, Galletta, \& King, 2005).

The proposed in-depth case study aims to contribute to the body of knowledge in cross-cultural IS research domain by investigating cultural differences at individual level while considering

\section{7. Theoretical models informing my research}

A research on micro phenomena in IS domain can and should be informed by more general macro theories on the nature of organisations and social processes within them (Walsham, 1995). The elements of theoretical grounding in my research were informed by the two main sources of literature: the first introducing various dimensions of culture; and the second emphasizing the multiplicity of diverse cultural influences received by an individual.

\section{Cultural dimensions by Hall}

In his book "Beyond Culture" anthropologist Hall (1976) argues that the human potential is strictly limited by an underdeveloped ability to get 'beyond culture' in order to achieve a more creative, expansive and responsive use of our capacities to think and communicate (verbally and non-verbally) and resolve problems (Tool, 1977). He concentrated on the communication patterns in various countries, suggesting two dimensions of culture: context and time.

Context is defined by Hall (1976) as the environment in which the communication process takes place. In high context cultures like Japan, a larger 
portion of the message is left unspecified and accessed through the context, non-verbal cues, and between-the-lines translations of what is actually said or written. In contrast, in the US, which is labelled as a low context culture, messages are expected to be explicit and specific (Limaye \& Victor, 1991). People in high context cultures tend to rely on visual elements and symbols, while people in low context cultures tend to rely on hard facts, data and statistics.

Communication in high-context cultures like Japan is more indirect and implicit and is more likely to use intermediaries because social harmony and face maintenance are crucial: using mediators eliminates face-to-face confrontation and reduces the risk of losing face (Jandt, 2001). On the contrary, communication in low-context countries like Germany and North America is highly structured, specific and tend to be very detailed, therefore good communication skills are highly valued as well as ability to express ideas logically.

Different impact of video channel on high and low-context cultures is explained by Olson and Olson (2003, p. 55) as follows:

"When people can see and hear each other, they can send and receive gestural and tonal signals. High-context cultures convey much of their message through tone and gesture. For them, the video channel is important. If people are from different cultures, however, there are two effects: (1) the gestural signals could be misread; and (2) if most of the message is in the gesture and intonation, high-context people are differentially hindered if they are without video. Low-context people have the habit of explaining context and being detailed and explicit. They might be as well off in conveying their message in audio as video; high-context people are likely to be hindered without video."

Concept of context is highly relevant to intercultural and development communication in general and development communication via videoconferencing in particular. For example, in high context cultures, providing too much information is considered talking down to others, whereas 
in low context cultures, doing so is considered being thorough. High context cultures appreciate slow, indirect messages whereas low context cultures insist on fast, direct messages. Critique exists, however, that Hall's context dimension is a not a rigorously developed model that has little or no empirical validation (Cardon, 2008; Kittler, Rygl, \& Mackinnon, 2011). Nevertheless, understanding the context model was a useful knowledge for interpreting and analysing the research data.

Time is an important and complex international variable in Hall's model of culture (Hoft, 1996). According to Hall (1959), perceptions of time in different cultures are either polychronic or monochronic. Polychronic time is characterized as simultaneous and concurrent, while monochronic time is characterized as being sequential and linear. In addition, people from polychronic cultures tend to engage in multiple tasks simultaneously and in a less organized manner, while people from monochronic cultures tend to do one task at a time, their manner well organized and chronological (Lee, Choi, Kim, Hong, \& Tam, 2004).

The monochronic or linear temporal orientation of much of Northern Europe and English-speaking North America and its effects on communication are fairly well known: members of these cultures come to the point very quickly with little introductory phrasing (Limaye \& Victor, 1991). By contrast, time is not linear in most other cultures; these polychronic cultures more frequently view time as flexible, their conversations and written communication can be more indirect or circular.

In an increasingly globalising world, when people from different cultures communicate in real time using advanced technologies, the old proverb "When in Rome, do as Romans do" simply doesn't work 
Table 2 Hall's dimensions from videoconferencing perspective

\begin{tabular}{|c|l|l|}
\hline \multicolumn{2}{|c|}{ Cultural Dimensions } & \multicolumn{1}{c|}{$\begin{array}{c}\text { Characteristics relevant to video-mediated } \\
\text { communication }\end{array}$} \\
\hline \multirow{2}{*}{ Context } & High & $\begin{array}{l}\text { Indirect and implicit communication, rely on gestural } \\
\text { and tonal signals, the video channel is important }\end{array}$ \\
\cline { 2 - 3 } & Low & $\begin{array}{l}\text { Structured and specific communication, rely on facts } \\
\text { and statistics, audio is as good in conveying message } \\
\text { as video }\end{array}$ \\
\hline \multirow{2}{*}{$\begin{array}{c}\text { Pime } \\
\text { Perception }\end{array}$} & Monochronic & $\begin{array}{l}\text { Get straight to the point, value quick response, good } \\
\text { communication skills and ability to express ideas } \\
\text { logically, 'time is money' }\end{array}$ \\
\cline { 2 - 3 } & Polychronic & $\begin{array}{l}\text { Long introductory phrasing, view time as flexible, find } \\
\text { direct approach as rude }\end{array}$ \\
\hline
\end{tabular}

\section{Cultural dimensions by Hofstede}

Hofstede (2001) defines culture as "the collective programming of the mind which distinguishes the members of one group from people from another". In other words, culture is not an individual characteristic, but rather a characteristic of a group of people shared by the members of that group, and which can eventually affect the behaviours and provide a norm to human behaviour (Lee, Choi, Kim, Hong, \& Tam, 2004). The majority of studies concerned with various cultural aspects of the development, implementation, use and management of IT have relied on Hofstede's (2001) model of national culture (Myers \& Tan, 2002).

The power distance cultural dimension focuses on the nature of human relationship in terms of hierarchy. It refers to the recognition and use of power in a society. Hofstede (2001', page 438) argues that there is a culture gap between developed and developing countries on the dimension of power distance: most donor countries score considerably lower on this dimension than the receivers, and the donor's representatives try to promote equality and democratic processes at the receiving end.

The individualism versus collectivism cultural dimension focuses on the relationship between individual and the group. Highly individualistic cultures believe that the individual is the most important unit, while highly collectivistic 
cultures believe that the group is the most important unit. Hosftede (2001) is convinced that there is always a culture gap between donor and receiving countries on the dimension of individualism/collectivism: donor countries, by definition rich, are culturally more individualist whereas receiving countries are more collectivist.

Uncertainty avoidance explains the willingness to cope with uncertainty and indicates the "extent to which people within the culture are made nervous by situations they perceive as unstructured, unclear, or unpredictable situations which they therefore try to avoid by maintaining strict codes of behaviour and a belief in absolute truth" (Hofstede, 1991)

Table 3 Hofstede's dimensions from videoconferencing perspective

\begin{tabular}{|c|c|c|}
\hline \multicolumn{2}{|c|}{ Cultural Dimensions } & Characteristics relevant to video-mediated \\
\hline \multirow{2}{*}{$\begin{array}{l}\text { Uncertainty } \\
\text { Avoidance }\end{array}$} & High & $\begin{array}{l}\text { Dependence upon specialists and expertise, } \\
\text { nervous in unpredictable situations, avoid risk }\end{array}$ \\
\hline & Low & $\begin{array}{l}\text { Risk taking, flexibility, have greater tolerance for } \\
\text { ambiguity }\end{array}$ \\
\hline \multirow{2}{*}{$\begin{array}{l}\text { Individualism } \\
\text { vs. } \\
\text { Collectivism }\end{array}$} & Individualistic & $\begin{array}{l}\text { Emphasis on individual initiative and } \\
\text { achievement, self-oriented }\end{array}$ \\
\hline & Collectivistic & $\begin{array}{l}\text { Emphasis on belonging, dependent on others, } \\
\text { collectively-oriented }\end{array}$ \\
\hline \multirow{2}{*}{$\begin{array}{l}\text { Masculinity } \\
\text { vs. } \\
\text { Femininity }\end{array}$} & Masculinity & $\begin{array}{l}\text { Large gender gap, emphasis on competitiveness } \\
\text { and material success }\end{array}$ \\
\hline & Femininity & $\begin{array}{l}\text { Small gender gap, emphasis on social welfare and } \\
\text { quality of life }\end{array}$ \\
\hline \multirow{2}{*}{$\begin{array}{c}\text { Power } \\
\text { Distance }\end{array}$} & High & $\begin{array}{l}\text { Top-down decision making, loyalty to the boss, } \\
\text { rigid hierarchical structure }\end{array}$ \\
\hline & Low & $\begin{array}{l}\text { More participative and democratic relationship } \\
\text { between superiors and subordinates. }\end{array}$ \\
\hline
\end{tabular}

The masculinity versus femininity cultural dimension focuses on gender roles, not physical characteristics. High-femininity countries blur the lines between 
gender roles, while high-masculinity countries display traditional differences in how age, gender, and family are viewed.

Later, Hofstede (1997) extended his work to include a fifth dimension - longterm versus short-term orientation to life - labelled as Confucian work dynamism. This dimension was first identified and called Confucian Work Dynamism by Michael Bond in a paper called Chinese Culture Connection (1987). According to this dimension, long-term orientation encourages thrift, savings, perseverance toward results, and a willingness to subordinate oneself for a purpose whereas short-term orientation is consistent with spending to keep up with social pressure, less savings, preference for quick results and a concern with face (Jandt, 2001). Probably because almost all references to Hofstede's work cite the original 1980 book where the four dimensions were initially defined (Ford, Connelly, \& Meister, 2003) my belief that the fifth dimension is also relevant to video-mediated cross-cultural communication found no confirmation in the literature.

Three important warnings raised for those who wish to follow Hofstede's research or use his model include: first, assuming that all members of a culture homogeneously carry the same cultural attributes, that a culture can be uniform; second, expecting individuals' values or behaviour to be wholly determined by their cultural background; and the third, confusing scores for cultural dimensions of culture with the constructs that they purport to measure - Hofstede's dimensions may be seen as manifestations of national culture, rather than as direct measures of national cultures (Williamson, 2002).

Authors within the IS field have questioned the overreliance on Hofstede's dimensions and characterization of culture (McCoy, Galletta, \& King, 2005; Myers \& Tan, 2002; Straub, 2002). The use of Hofstede's country scores, now over 30 years old, can no longer be assumed to be representative of the views of all individuals from a given country (McCoy, Galletta, \& King, 2005). Myers and Tan (2002) expressed two major concerns regarding Hosftede's taxonomy of culture: firstly, the dimensions assume culture falls along national boundaries and that the cultures are viewed as static over time and secondly, 
national culture is assumed to be homogenous; subcultures are often assumed to not exist in the Hosftede's model of culture.

"I had made a paradigm shift in cross-cultural studies, and as Kuhn (1970) has shown, paradigm shifts in any science meet with strong initial resistance" writes Hofstede (2001', page 73) in response to the criticism. His answers to the main comments on his model are presented on the next page.

Table 4 Hofstede's (2002) response to the criticism of his model

\begin{tabular}{|l|l|}
\hline Criticism & Response \\
\hline $\begin{array}{l}\text { Surveys are not a suitable way } \\
\text { of measuring cultural } \\
\text { differences }\end{array}$ & They should not be the only way \\
\hline $\begin{array}{l}\text { Nations are not the best units } \\
\text { for studying cultures }\end{array}$ & $\begin{array}{l}\text { True, but they are usually the only kind of } \\
\text { units available for comparison and better } \\
\text { than nothing }\end{array}$ \\
\hline $\begin{array}{l}\text { A study of the subsidiaries of } \\
\text { one company cannot provide } \\
\text { information about entire } \\
\text { national cultures }\end{array}$ & $\begin{array}{l}\text { Any set of functionally equivalent samples } \\
\text { from national populations can supply } \\
\text { information about cultural differences }\end{array}$ \\
\hline $\begin{array}{l}\text { The IBM data are old and } \\
\text { therefore obsolete }\end{array}$ & $\begin{array}{l}\text { The dimensions found are assumed to have } \\
\text { centuries-old roots; only data which } \\
\text { remained stable across two subsequent } \\
\text { surveys were maintained }\end{array}$ \\
\hline $\begin{array}{l}\text { Four or five dimensions are } \\
\text { not enough }\end{array}$ & $\begin{array}{l}\text { Additional dimensions should be both } \\
\text { conceptually and statistically independent } \\
\text { from the five dimensions already defined. } \\
\text { Researchers are welcome to develop new } \\
\text { dimensions. }\end{array}$ \\
\hline
\end{tabular}

It is possible for researchers to use the definitions of the national culture dimensions to study cultures in a qualitative manner although the ontological perspectives of interpretivist paradigm might not be congruent with the assumptions of Hofstede's dimensions (Ford, Connelly, \& Meister, 2003). Therefore, I searched for an alternative theoretical underpinning for my interpretive inquiry and derived to the conclusion that the Social Identity theory is an appropriate candidate. 


\section{Social identity theory}

According to Social Identity Theory (SIT) people tend to classify themselves and others into various social categories, such as organisational membership, religious affiliation, gender and age cohort (Tajfel \& Turner, 1985) where categories are defined by prototypical characteristics abstracted from the members (Turner, 1985). Social classification serves two functions:

- First, it cognitively segments and orders the social environment, providing the individual with a systematic means of defining others;

- Second, it enables the individual to locate and define him- or herself in the social environment (Ashforth \& Mael, 1989).

We use social categories like black or white, Russian or Chinese, Christian or Muslim, student or housewife, single or married, taxi driver or doctor, buyer or seller, because they are useful. If we can assign people to a category then that tells us things about those people. Similarly, we define appropriate behaviour by reference to the norms of groups we belong to, but one can only do so if he or she knows who belongs to this group. For example, I can define myself as an Asian woman, married with children, a PhD candidate at Victoria University of Wellington, currently in New Zealand. Perceiving myself as a member of postgraduate community at Victoria University, I think and act adequately; knowing that I'm a mother I feel and perform as one; most of the time I communicated in English when I lived in New Zealand.

SIT recognizes that the various identity groups with which an individual is affiliated (e.g., gender, race, generation, occupation) have relative levels of importance on different beliefs and behaviours. Certain social identity layers matter in specific circumstances, but, in general, it makes little sense to attempt to hierarchically order these layers. (Gallivan \& Srite, 2005).

SIT also proposes that people strive to achieve or maintain a positive social identity (thus boosting their self-esteem), and that this positive identity derives largely from favourable comparisons that can be made between the ingroup and relevant outgroups: in the event of an 'unsatisfactory' identity, people may seek to leave their group or find ways of achieving more positive 
distinctiveness for it (Brown, 2000). Social identification is, therefore, is the perception of oneness with or belongingness to some human aggregate (Ashforth \& Mael, 1989).

Following Hofstede's (2001', p. 11) description of the manifestations of culture as layers around a core of values, Straub and co-authors (2002) take this a step further and use an analogy of a 'virtual onion', where the layers are permeable and do not have a given order or sequence, to convey the complexity and lack of predictability of an individual's cultural characteristics (ibid, page 14).

Based on SIT, Straub et al. (2002) describe and illustrate a new conceptual framework about culture which they believe will help researchers to understand the complex and rich implications of individuals' social identity. The underlying assumption of their view is that an individual's social identity represents a combination of cultures across boundaries (national, organisational, professional, etc.) which blend together to create one's overall culture.

The 'virtual onion' metaphor suggests that, like layers of an onion, each individual contains different 'layers' of cultural identity and experiences, but that the sequence of these layers are virtual, in the sense that they can shift, depending on time and circumstances (Gallivan \& Srite, 2005), see Figure [7].

Figure 6 The “Onion Diagram” Manifestations of Culture at Different Levels of Depth (Hofstede, 2001', page 11)

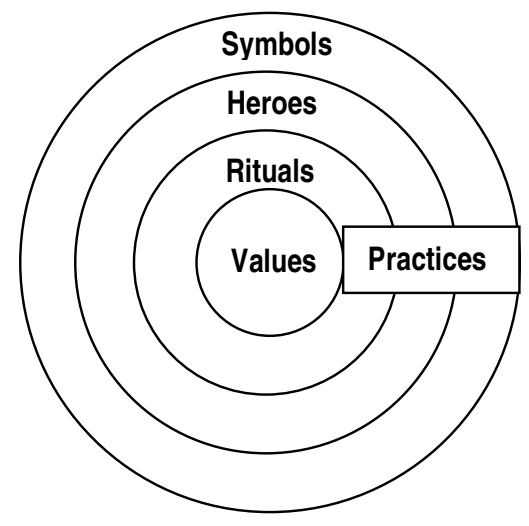


Figure 7 The “Virtual Onion Model” (Gallivan \& Srite, 2005; Karahanna, Evaristo, \& Srite, 2005; Straub, 2002)

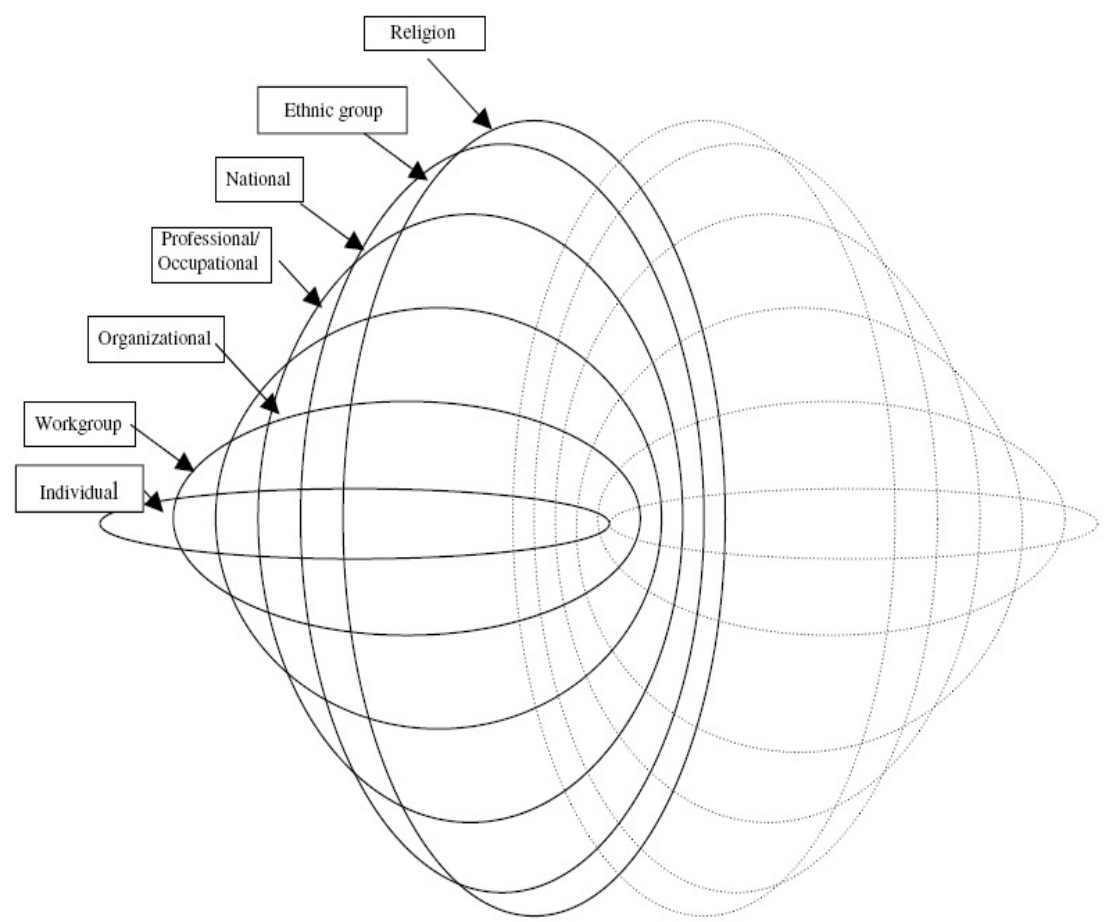

Various levels of culture are both hierarchically and laterally related (Karahanna, Evaristo, \& Srite, 2005). These interrelations can be seen in Figure 7: it shows an individual (the innermost ellipse composed of a solid line) who is a product of several layers of culture interacting with other individuals and their layers of culture (the dotted ellipses) (Gallivan \& Srite, 2005). For example, ethnic culture can span national boundaries (such as people of Chinese descent having immigrated but somewhat kept the culture of their home country, a very good example is a NZ-made documentary "Banana in a Nutshell"), but ethnic culture may also represent a subset of individuals in a single country (Maoris in New Zealand or Kazaks in Mongolia).

Similar arguments can be made for religious, linguistic, and other levels of culture; relationship across levels of culture is not necessarily hierarchical from the more general to the least general (Karahanna, Evaristo, \& Srite, 2005). For instance, in the case of multinational corporations or multilateral development agencies, organizational culture can span national, religious, ethnic, regional, linguistic, and professional cultures. Furthermore, various groups, such as 
participants of a GDLN event, may include members from several organizations, professions, nations, religions, ethnic backgrounds, and linguistic backgrounds

The virtual onion metaphor is a more abstract representation of culture than those commonly used in the IS literature: it operates at the individual level to reflect the multiple, complex forces that shape individuals' beliefs and behaviour (Gallivan \& Srite, 2005). Researchers are encouraged to consider the virtual onion model, derived from SIT, as an alternate conceptualization that will be explicit about the many social identity layers that shape individuals' beliefs and behaviour (Gallivan \& Srite, 2005).

Following this advice, the proposed research will be carried out under the assumption that each individual has a unique social identification that influences his/her perceptions, understanding, and interpretation of information and knowledge in the process of communication.

\section{8. Gaps in the knowledge and research questions}

There is extensive literature on distance learning and organisational communication that occurs through the videoconferencing channel. A wide body of literature in IS domain explores the impact of cultural differences on the development and use of information and communication technologies. Many IS scholars measured the perceived effectiveness of various information and communication technologies. With regard to development communication scholarship, again there is a large volume of research on how broadcast oriented media channels such as one-way video streaming was used for communicating ideas of social change. But none of the existing literature in these domains answers the research questions that are formulated as:

What cross-cultural communication factors influence the effectiveness of videoconferencing on development issues as perceived by the participants?

- How do the cultural differences influence the effectiveness of videoconferences in perceptions of participants?

- How can the factors identified be used to bridge these cultural differences for more effective communication? 
This review has established a gap in the literature in the area of the use of videoconferencing technology in cross-cultural communication on development. Moreover, no empirical evidence of in-depth interpretive exploration of perceived effectiveness of videoconferencing in the context of development was found in the literature. The development communication field lacks research that investigates the use of new communication technologies such as videoconferencing in facilitating effective communication for development (Wilkins \& Mody, 2001); at the same time, IS field needs more cross-culturally framed research exploring the impact of cultural differences on the use of ICT including videoconferencing (Hunter, 2001; Myers \& Tan, 2002). Practitioners know that cultural differences exist, what they would like to know is how to bridge these cultural differences for more effective communication (Pauleen, Evaristo, Davison, \& Ang, 2006).

The answers to the proposed research questions will correspond neatly with an apparent gap in the academic literature and while doing so, meet the needs of practitioners.

\section{9. Chapter summary}

This chapter introduced the findings from an extensive literature review in the field of intercultural communication, videoconferencing and its perceived effectiveness from the distance learning, organisational communication and development communication perspective. It also reviewed the current crosscultural research in information systems domain and introduced the theoretical models that inform this research. It concludes that gaps in the knowledge will be addressed by the proposed research. 


\section{Chapter 3: Research methodology}

The interpretive perspective is chosen for this research and this is complemented by the use of case study as the methodology. These particular approaches are suitable for the research questions that I have posed and will allow me to investigate the nature of technology-mediated cross-cultural communication and understand the specific role of culture as a factor influencing the effectiveness of this interaction. The topic domain represents a relatively new type of intercultural communication that has not been covered in IS research - communication on development agenda.

The multiple case study design adopted in my study affords the opportunity to examine human perceptions and experiences in order to answer the research questions:

What cross-cultural communication factors influence the effectiveness of videoconferencing on development issues as perceived by the participants?

- How do the cultural differences influence the effectiveness of videoconferences in perceptions of participants?

- How can the factors identified be used to bridge these cultural differences for more effective communication?"

This chapter provides a rationale for choosing the interpretive perspective and a qualitative methodology, introduces the proposed research design and describes research method that will be used for the collection, analysis and interpretation of research data associated with this study. My researcher's role is described and mitigations are noted for a number of potential biases followed by acknowledgment of various limitations. A timeframe for the study concludes this chapter.

\section{1. Ontological and epistemological stance}

When establishing knowledge about an aspect of reality, every researcher has to make specific assumptions about the nature of reality under investigation ontology and about the nature of knowledge - epistemology (Sandberg, 2005). 
In terms of ontology, Archer (1988) identified three broad positions: the first, 'external realism' proposes that reality is objective and it exists independently of individuals; the second, 'internal realism' considers reality as an intersubjective construction shared between individuals; while the third, 'subjective idealism' sees reality as a personal construction of each individual (Nandhakumar \& Jones, 1997). Interpretive researchers usually would adopt one of the latter two positions, particularly with regard to the human interpretations and meanings associated with computer systems (Walsham, 1995). But in doing so, researchers must be aware of solipsism - an extreme form of subjective realism - according to which there exists only one subject: that which I constitute myself and according to which this ' $\mathrm{I}$ ' is 'alone' and 'unique' (Stern, 1948). I disassociate my research from this approach.

The ontological stance adopted in my research will be clearer to the reader if I explain my decision following an example used by Sandberg (2005). The primary research object in this study is effectiveness of cross-cultural communication (that occurs via video channel). I interviewed participants of videoconferencing events in order to understand their perceptions about effectiveness of video-mediated communication. In order to be able to interpret lived experiences of my respondents, I must have a very clear understanding of what inter- and cross-cultural communication and technology-mediated communication means. This understanding is my - as a researcher interpretation of what intercultural video-mediated communication means to the participants interviewed. While reading through the interview transcripts, I might experience a discrepancy between my initial interpretation and the way my respondents perceive the effectiveness of cross-cultural video-mediated communication. In this case, the truth is not evident. Based on the first reading, I will formulate a new interpretation and will read the transcripts a second time. As data collection progresses, my initial understanding about the object of study will evolve. This iterative process will continue until an agreement is reached between my presumed interpretation of effectiveness and the way the respondents perceive it. Only then can the knowledge achieved be claimed as truth. I may therefore conclude that my research will adopt the second position 
of 'internal realism' that views reality as an inter-subjective construction of the shared understanding of individuals. However, this research does not limit itself to the study of participants' meanings only, it will be an interpretation of the phenomena through my, researcher's lenses.

Epistemology - the theory of knowledge - refers primarily to three central questions for the researcher: 1) how can individuals achieve meaning and thereby knowledge about the reality in which they live? 2) how is this knowledge constituted? and 3) under what conditions can the knowledge achieved be claimed as true? (Sandberg, 2005).

With respect to epistemology, Archer (1988) identifies three alternative positions in relation to the nature of knowledge claims: 'positivism', proposing that the social world can be described in terms of law-like generalisations and that knowledge can therefore be acquired through the collection of pure valuefree facts; 'anti-positivism' which proposes that facts and values cannot be separated and both involved in scientific knowledge, and 'normativism' which sees scientific knowledge as ideological, serving the interests of particular social groups (Nandhakumar \& Jones, 1997). Following Walsham's (1995) suggestion that either of the latter two positions may be adopted by interpretive researchers, I believe that the proposed study takes the anti-positivist epistemological stance.

\section{2. The choice of an interpretive research philosophy}

The nature and complexity of the phenomena investigated by behavioural information systems researchers would be better informed by a plurality of research paradigms (Orlikowski \& Baroudi, 1991). "Paradigm' is the most fundamental set of assumptions adopted by a professional community that allows its members to share similar perceptions and engage in commonly shared practices (Hirschheim \& Klein, 1989). Alternatively, the Oxford English

Dictionary defines paradigm as "a pattern or model, an exemplar". Based on the underlying research epistemology, Orlikowski and Baroudi (1991), following Chua (1986), suggest three 'paradigms' for qualitative research: positivist, interpretive and critical. 
IS research can be classified as positivist if there is evidence of formal propositions, quantifiable measures of variables, hypothesis testing, and the drawing of inferences about a phenomenon from a representative sample to a stated population (Klein \& Myers, 1999). Critical studies aim to critique the status quo and remove contradictions from organizations and society by exposing the deep-seated structural problems; such studies that are concerned with evaluation, as well as with description and explanation (Orlikowski \& Baroudi, 1991).

IS research can be classified as interpretive if it is assumed that our knowledge of reality is gained only through social constructions such as a language, shared meanings, documents, tools, and other artifacts. Interpretive research does not predefine dependent and independent variables, but focuses on the complexity of human sense making as the situation emerges; it attempts to understand the phenomena through the meanings people assign to them (Klein \& Myers, 1999).

The development of the interpretive empirical school in IS has not been free of controversy fuelled by debates on the relative merits of interpretivist versus positivist approaches to IS (Walsham, 1995) but now it is a well-established part of the field (Walsham, 2006).

The word 'qualitative' is not a synonym for 'interpretive' - qualitative research may or may not be interpretive, depending upon the underlying philosophical assumptions of the researcher (Myers, 1997). As a result, a researcher's choice of a specific qualitative research method can be independent of the underlying philosophical position adopted. For example, the case study method could be positivist, interpretive or critical.

A qualitative researcher in IS either adopts an interpretive stance aiming towards an understanding that is appreciated for being interesting; or a pragmatist stance aiming for constructive knowledge that is appreciated for being useful in action (Goldkuhl, 2012). This study is not a simple description of participants' subjective meanings separate from the context, it is rather my - as a researcher - interpretation of the process of cross-cultural video-mediated communication that is investigated in its natural environment. I hope this 
research will be appreciated by the researchers and practitioners working in the field as being interesting.

Given the complexity of the phenomena of cross-cultural communication mediated by videoconferencing technology, I have chosen the interpretive approach because it will help me to understand human thought and action in social and organisational contexts and it has the potential to produce deep insights into information systems phenomena (Klein \& Myers, 1999).

\section{3. Rationale for the choice of case study}

A case study is an empirical inquiry that (1) investigates a contemporary phenomenon within its real-life context, especially when (2) the boundaries between phenomenon and context are not clearly evident (Yin, 1994). It is a one of the more interactive methods of data collection and makes a deeper understanding of individual factors possible (Yin, 2003). It allows the researcher to ask "how" and "why" questions, which helps understand the nature and complexity of the processes taking place (Benbasat, Goldstein, \& Mead, 1987). Case study is also more suitable to collect data that reflects the interrelationships between the involved factors and actors, because of the data richness and openness of asked questions (Linton, 2002).

A case study approach is deemed appropriate if: (1) the phenomenon of interest cannot be studied outside its natural setting, (2) the study focus on contemporary events, (3) control or manipulation of subjects or events is not possible, and (4) the phenomenon of interest does not enjoy an established theoretical base (Benbasat, Goldstein, \& Mead, 1987). The first three requirements are fulfilled for the proposed research; the fourth one might be debatable because use of videoconferencing technology

Convinced that the above qualities will facilitate achievement of research objectives, I have chosen a case study method in my attempt to understand the perceptions of participants about the effectiveness of cross-cultural communication that occurs via videoconferencing. 
Researchers who have successfully applied case study method in the areas similar to my topic include Webster (1998) on desktop videoconferencing, Walsham (2002) on cross-cultural communication, Dennis and Garfield (2003) on perceived effectiveness of mediated communication, and Jacobson and Storey (2004) on implementation of participatory communication programs in Nepal. Examples of successful application of case study method by $\mathrm{PhD}$ candidates include an inquiry on teaching and learning through broadband networks and IP-based videoconferencing (Andrews, 2005) and on setting up an internet-based videoconferencing for establishing a cross-cultural learning collaboration between Chinese school and Canadian school (Chen, 1999).

One major shortcoming of the method, whether the research involves single case studies or multiple-case studies, has been considered a difficulty in generalizing case study findings. Yin (1999) proposes a solution: "the remedy is to consider a case study, as a unit, to be equivalent to an experiment, as a unit; multiple-case studies may then be considered equivalent to multiple experiments. Under this assumption, the problem of generalizing from case studies is no different from the problem of generalizing from experiments where hypotheses and theory are the vehicles for generalization. To this extent investigators doing case studies are not "theory driven", but are "driven to theory" (page 1212). Prominent researchers such as Walsham (1995), Lee and Baskerville (2003) have comprehensively refuted those who say that 'you can't generalize from a case study'.

The description of field data obtained from the proposed cross-cultural comparative case study can be generalised to the other centres in GDLN (Lee \& Baskerville, 2003). For example, the presentational data (what the interviewees tell me in answer to my questions aimed to understand their perceptions regarding the effectiveness of GDLN events) and operational data (what I observe in their actions and behaviours during videoconferences and interviews) can be generalised into an empirical statement that can be transferred to some other centres within this particular development learning network or at least to those located in the East Asia and Pacific region of GDLN. 


\section{4. Research strategy}

The exploratory strategy adopted in this study originated from my research question that seeks to discover the factors that influence the effectiveness of cross-cultural communication mediated by videoconferencing technology. Moreover, this study aims to answer this question in the context of crosscultural communication on development topics that occurs during GDLN events. This study is exploratory in purpose because the literature is relatively silent on the phenomena of video-mediated cross-cultural communication in the context of development cooperation. In comparison, the following fields that are also relevant to my study, have been well explored:

- The problem of cross-cultural communication (Hall, 1976; Hofstede, 2001) in general and technology-mediated cross-cultural communication (Guo \& D'Ambra, 2003; Kim \& Bonk, 2002; Lee \& Lee, 2003; Leidner \& Kayworth, 2006) in particular.

- The field of organisational communication mediated by videoconferencing technology (Campbell, 1999; Fulk \& Dutton, 1984; Gump, 2006; Panteli \& Dawson, 2001; Peszynski \& Yoong, 2002; Webster, 1998).

- The area of video-mediated distance learning (Arnold, Cayley, \& Griffith, 2002; Brown, Rietz, \& Sugrue, 2005; Carville \& Mitchell, 2000; Clarke, Butler, Schmidt-Hansen, \& Somerville, 2004; Knipe \& Lee, 2002; Middlehurst \& Woodfield, 2006).

- The field of development communication that refers to a process of strategic intervention toward social change initiated by institutions and communities (Holden \& Gale, 1993; Kim, 2005; Melkote \& Steeves, 2001; Wilkins \& Mody, 2001).

\section{5. Design of the case study}

Although 'the proof of the pudding is in the eating, attention need to be paid to the recipe' or in other words, a well defined research design will help researchers to establish a clear chain of evidence in advance of their fieldwork or to think through how to proceed from data to explanations of findings (Vries \& Roest, 1999). 
Yin (2003) distinguishes four types of research designs: single-case (holistic); single case (embedded); multiple-case (holistic) and multiple-case (embedded). A single case study is an appropriate design under the following circumstances: 1) when the case represents the critical case in testing a well-formulated theory; 2) when the case represents an extreme or a unique case; 3) when case is the representative or typical case; 4) when a single-case study is a revelatory case, that is when an investigator has an opportunity to observe and analyse a phenomenon previously inaccessible to scientific investigation; and 5) when a single-case study is the longitudinal case, studying the same single case at two or more different points in time (Yin, 2003).

The same case study may involve more than one unit of analysis, where the main unit is the organisation as a whole; intermediary unit is a department and the smallest unit is an individual so these sub-units are embedded into the same case. For example, Panteli and Dawson (2001) studied the implementation, training and use of videoconferencing systems on different sites of a multinational oil company. In contrast, if the case study examines only the global nature of an organisation or of a program, a holistic approach would have been taken. Jacobson and Storey (2004) applied a single-case design in their study of a population communication program carried out by the government of Nepal with the support of international aid organisations. Single cases allow researchers to investigate phenomena in depth and provide rich description and understanding (Walsham, 1995).

The same study may contain more than a single case. When this occurs, the study has used a multiple-case design, and such designs have increased in frequency (Yin, 2003). Doherty and Doig (2003) adopted a multiple case study approach for studying whether changes in the flow of information were likely to engender cultural changes, within a variety of large and highly sophisticated commercial organizations.

The multiple-case embedded design has been chosen for my study which aims to gain the necessary in-depth interpretation of the anticipated impacts of 
cultural differences of participants upon the perceived effectiveness of videoconferencing events.

\section{Unit of analysis}

Yin (2003) provides a general guidance that the unit of analysis should be defined in relation to the initial research questions. Since my research aimed to understand people's perceptions, the unit of analysis in my research was an individual who participates in videoconferencing events. The choice of an individual as a unit of analysis allowed me to build my enquiry on the Virtual Onion Model (Gallivan \& Srite, 2005; Karahanna, Evaristo, \& Srite, 2005; Straub, 2002) based on Social Identity theory (Tajfel \& Turner, 1985).

This research has largely relied on the data obtained through interviews because people are normally willing to talk about themselves with reasonable openness and honesty, provided that they perceive the researcher's sincerity of interest, feel that they understand the researcher's agenda, and trust the researcher's statements on confidentiality (Walsham, 2006). This is important because in reporting the findings, researchers often offer a single illustration in the participant's own words as a result of editorial challenges to include sufficient qualitative data in the final manuscript (Drisko, 1997).

An individual unit of analysis was appropriate in my study because I aimed to understand participant's unique set of values that has been formed as result of complex influence of diverse cultures and sub-cultures (e.g. national, organisational, professional), and their particular behaviours and perceptions affected by their differing backgrounds (Straub, 2002). For example, participants of a GDLN event taking place in Canberra were of different nationalities (Australians, Indonesians, Vietnamese, Timorese, American, etc.), belonged to different stakeholder groups (government officials, NGO activists, students and professors), played different roles in this event (presenter, moderator, facilitator, regular participant), etc. They were men and women of different ages, with different educational backgrounds and social status, belonged to different professional, political, religious or other groups. A complex blend of these different layers of culture possessed by each individual 
influenced his or her perceptions about effectiveness of GDLN videoconferences.

\section{Number and selection of cases}

Sampling decisions in cross-cultural studies involve two distinct levels: sampling of cultures and sampling of subjects (van de Vijver \& Leung, 1997). Many studies involve a convenience sample of cultures, typically ones where the researcher has pre-established contacts; this strategy reduces the considerable costs of conducting cross-cultural research (Karahanna, Evaristo, \& Srite, 2002).

In order to achieve an understanding of perceived effectiveness in videoconferencing in the context of GDLN, I examined several videoconferencing events that took place in DLCs at four different locations. Two field sites represented a community of GDLN affiliates that are located in the developed countries: New Zealand DLC hosted by Victoria University of Wellington and Australia DLC hosted by the Crawford School of Economics and Government, Australian National University. The larger population of affiliates in the less developed part of the world has been represented by Ulaanbaatar DLC in Mongolia and two DLCs in Moscow, Russian Federation.

I have selected the above two sites for the following reasons. The newly emerging New Zealand DLC is hosted by Victoria University of Wellington, so it was possible to collect data with no costs involved. This case also became an example of an early stage of adoption of videoconferencing for communication on development by a GDLN university-based affiliate. The Australian DLC was chosen due to geographical proximity to New Zealand but also because this DLC has been affiliated to GDLN for a number of years and thus have greater experience in videoconferencing.

The sites in Ulaanbaatar and in Moscow represented the larger population of GDLN affiliates scattered throughout the developing world. Ulaanbaatar DLC is located in my home country. Moreover, I have been working as a Training Coordinator at this centre and had an easy access to the materials as well as benefited from extensive list of personal contacts. 
Selection of the second field site in the Russian Federation was considered for two reasons: a) I can communicate fluently in Russian and was able to develop research instruments and gather first-hand (not translation-mediated) rich information from interviews and other sources; b) research fieldwork in Russia did not impose high demands on the research funds due to a geographical proximity to my home country, Mongolia.

The second location was also chosen because three out of 12 currently active affiliates in the Russian Federation are located in the capital city of Moscow. Although I expected that there will be greater chances to observe more videoconferences in two organisations in Moscow because normally DLCs located in other Russian cities would have fewer videoconferences, maximum of one event in a week. Unfortunately, I arrived in Moscow in August when most of the people were away enjoying holidays and no videoconferences took place because of that.

The two countries representing GDLN affiliates located in developing countries - Russian Federation and Mongolia - represented former socialist model societies. Russia was the largest republic of the former Soviet Union and is a collection of diverse territories at different stages of development. The country achieved unprecedented macroeconomic stability since the 1998 crisis: important reforms facilitated the rapid development of market institutions in many areas. Mongolia also has made substantial progress over the past decade of democratic restructuring in laying down sustainable macroeconomic foundations and creating a private sector-led open economy. I therefore made an assumption that data obtained from interviews will have common grounds in terms of people's mindset and similar level of economic development. My assumptions proved to be right, indeed. I expect that the research findings can be generalisable to other post-soviet countries in Europe and central Asia.

Then the question may arise: if Russia and Mongolia are so similar, why to collect data in both countries? The short answer is: the two counties are tremendously distinct therefore each case contributed a rich cultural insight into this cross-cultural comparative study. Russia stretches over a vast expanse of Asia and Europe and its culture is a hybrid created from the cultures of the 
nationalities of this multinational state. Despite the heavy, almost 70-years-long Soviet influence, Mongolia is an Asian country with its immense historical and cultural heritage.

The two countries representing GDLN affiliates located in the developed part of the world - Australia and NZ - also share common values, have modern, prosperous and industrialised economy, with populations mostly of European descent. Contemporary New Zealand has a diverse culture with influences from English, Scottish, Irish, and Maori and Polynesian cultures. Australian culture until the mid-20th century was British and/or Irish, but over the past 50 years, it has been strongly influenced by American culture, as well as largescale immigration from non-English-speaking countries. Again, both countries added significant cultural implications to this study and the findings can be generalised to other countries in the developed part of the world.

Selection of locations for the fieldwork was limited by two important factors: time and cost. However, it did not affect the quality of information and credibility of the study because selected cases provided adequate quality and quantity of information to manifest the videoconferencing phenomenon from two perspectives (developing countries and developed countries) and on the example of both well established and less experienced centres on each side. 
Figure 8 Number and selection of cases

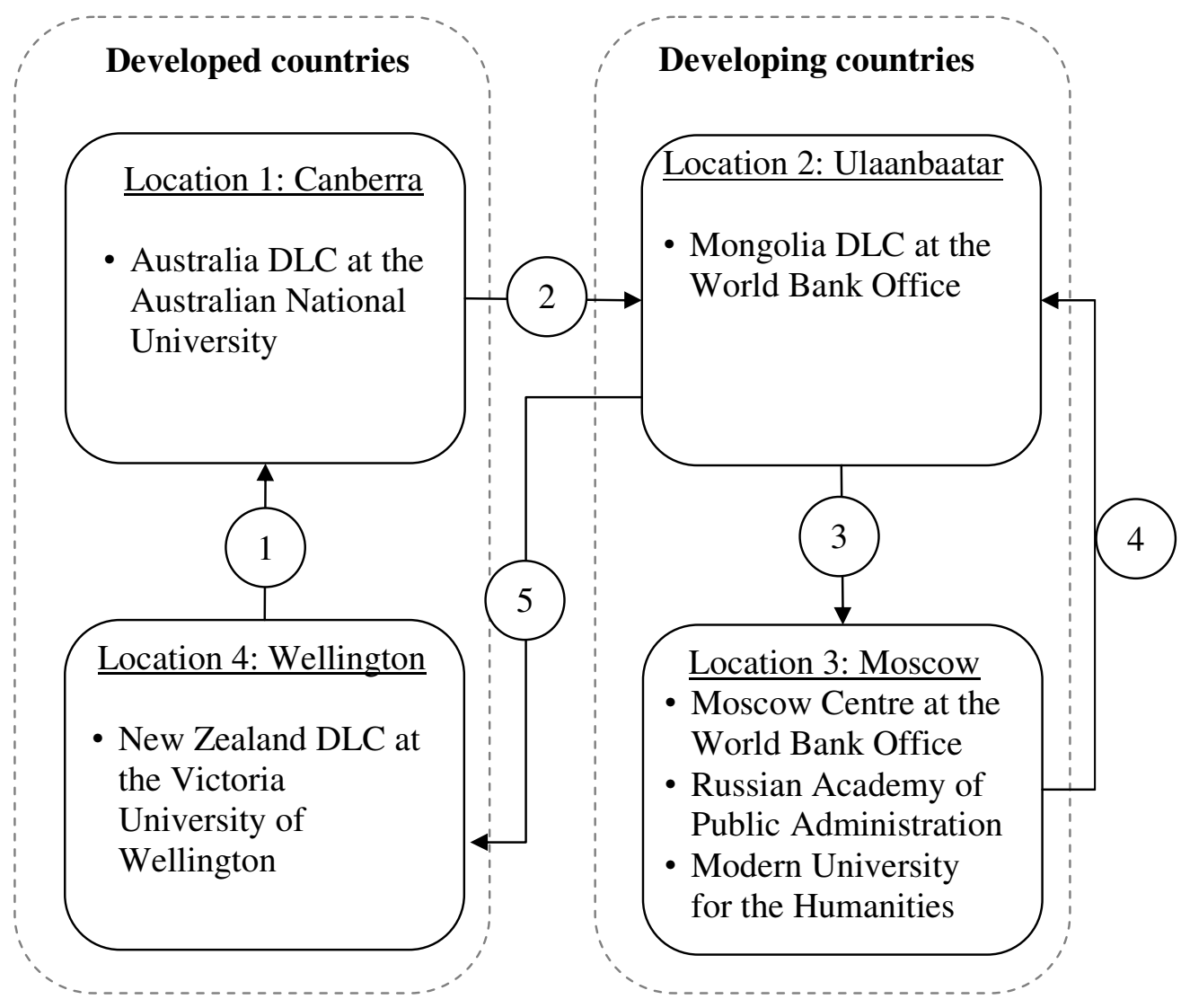

The numbers on the arrow connecters indicate the sequence of fieldwork travel: it started in Canberra (3 weeks), continued in Moscow (3 weeks) after a weeklong break in Mongolia and proceeded to the Ulaanbaatar-based case. The Wellington-based case has completed the fieldwork.

\section{Use of a case study protocol}

The protocol is intended to guide the investigator in carrying out the collection of relevant data, keeps him or her targeted on the subject of the case study, helps to anticipate problems and facilitate productive work in the field (Yin, 2003).

The observational protocol for recording of observational data obtained during my fieldwork consisted of descriptive notes (for example, number and profile of participants, description of the videoconferencing setting, etc.) and reflective notes (my personal thoughts, ideas, impressions, etc.). This document also 
included demographic information about the time, place and date of the event where the observation took place.

The interview protocol helped me to follow the basic structure of the interview, ensure good coverage of the research question, efficiently use the interview time, and be prepared for unanticipated situations and surprise questions.

Yin (2003) emphases that the heart of the case study protocol is a set of substantive questions reflecting the actual line of inquiry posed to the researcher. He adds that these questions are intended to keep the investigator on track as data collection proceeds and serve as reminders regarding the information that needs to be collected and why. The following six levels of questions can be identified in my inquiry:

Level 1: Questions that guided my actions at the level of an individual interview. What is the participant's role in a videoconference? What subcultures they belong to? Have they had previous distance learning experience?

Level 2: Questions I had to find answers for at the level of an individual case. For example, I worked at five DLCs in four countries. The Mongolian case included questions about one DLC whereas in Russia I worked on two cases so the questions at this level were applicable to the two DLCs located in Moscow. For example: Is this Centre well established? How experienced are people working there? How many events have they organised? Who are their regular participants?

Level 3: Questions asked of the pattern of findings across pairs of locations: cases representing DLCs in Australia and New Zealand and cases representing DLCs in Mongolia and Russia. At this level, I searched for cross-case patterns looking for within-pair similarities and cross-pair differences from several dimensions (Eisenhardt, 1989): type of DLC (co-located with the World Bank office vs. hosted by a university), experience (well established vs. new), topic priorities, etc.

Level 4: Questions asked of the pattern of findings across all five cases in four different cultures. What are the similarities and differences across all cases? 
How different are the perceptions of presenters, facilitators, moderators, translators and regular participants across all cases?

Level 5: Questions asked of an entire study. What are the similarities in perceptions of participants in this study compared to studies that investigated effectiveness of desktop videoconferencing? Will this study enhance our understanding of cross-cultural communication about and for development?

Level 6: Normative questions about policy recommendations and conclusions such as: What is the anticipated outcome of this investigation? Will the findings be generalizable to other DLCs within GDLN in general or its regional networks in particular? Will the findings be applicable to other videoconferencing networks?

The first four levels of study questions guided the entire data collection process whereas the last two levels of inquiry questions helped me to maintain a broad perspective and prevent from collecting data for the sake of it.

\section{6. The data gathering process}

\section{Access to fieldwork sites}

My first objective in selecting Distance Learning Centres to participate in this cross-cultural study was to gain access to GDLN affiliates which represent different countries and regions, varying level of maturity within the network, associate with a range of stakeholder groups and contribute to the GDLN community in a variety of ways.

The first and the most obvious choice was Mongolia DLC in Ulaanbaatar because the New Zealand Development Scholarship - of which I was a recipient - covers costs associated with the conduct of research fieldwork in student's home country. Moreover, I have been working as a Training Coordinator at this centre and therefore have easy access to the materials as well as extensive circle of personal contacts among regular participants of videoconferencing events.

Selection of the second field site in the Russian Federation was made for two reasons: firstly, I communicate fluently in Russian and therefore was able to 
interview the respondents in their native language. According to Myers \& Newman (2007), an interviewer should be able to create space for the interviewee to reveal their personality and identity. Speaking Russian with individuals that have kindly agreed to participate in my research enabled me to create a comfortable and candid atmosphere during interviews as well as to gather first-hand (not translation-mediated) quality information. Secondly, a research fieldwork in Russia did not impose high demands on the research funds due to a geographical proximity to my home country, Mongolia: a student fare for return flight from Ulaanbaatar to Moscow via MIAT Mongolian Airlines was less than NZ\$600. Also, thanks to my Russian friends I was able to arrange a safe and reasonably priced accommodation in Moscow - the world's most expensive city for according to Mercer's 2008 Cost of Living Survey (ByeByeBlighty.com, 2008).

The second location was also chosen because three out of a dozen currently active affiliates in the Russian Federation are located in the capital city of Moscow. However, after my arrival to Moscow I discovered that one of the affiliated organisations - International Institute of Management LINK - in fact did not have own videoconferencing facilities. Despite my assuming that there would be a greater chance for me to observe more videoconferences at two DLCs in Moscow, the reality was that I observed no live videoconferencing events in Moscow because I arrived there in August - the "quiet" month in the capital city when most of the people are away on holidays and therefore the Moscow DLCs were not signing up for ongoing GDLN events. However, I was given a DVD with recordings of some videoconferencing events.

The DLCs in Ulaanbaatar and in Moscow represent the larger population of GDLN affiliates scattered throughout the developing world and located in the former socialist model societies. Russia was the largest republic of the former Soviet Union and is a collection of diverse territories at different stages of development. The country achieved unprecedented macroeconomic stability since the 1998 crisis: important reforms facilitated the rapid development of market institutions in many areas. Mongolia also made substantial progress over the past decade of democratic restructuring in laying down sustainable 
macroeconomic foundations and creating a private sector-led open economy. I therefore made an assumption that data obtained from interviews will have common grounds in terms of people's mindset and the data supports this assumption (see Chapter 5). The research findings thus can be generalisable to other post-soviet countries in Europe and central Asia.

The newly emerging New Zealand DLC hosted by Victoria University of Wellington is the third location of my research. Obviously, it was possible to collect data with almost no costs involved. This DLC is an example of an early stage of adoption of videoconferencing for communication on development by a GDLN university-based affiliate.

The fourth organisation - Australia DLC hosted by the Crawford School of Economics and Government, Australian National University - was chosen due to geographical proximity to New Zealand and also because it has been affiliated to GDLN for a number of years thus have greater experience in videoconferencing.

The two countries representing GDLN affiliates located in the developed part of the world - Australia and NZ - also share common values, have modern, prosperous and developed economy, with population mostly of European descent. Contemporary New Zealand has a diverse culture with influences from English, Scottish, Irish, and Maori and Polynesian cultures. Australian culture until the mid-20th century was British and/or Irish, but over the past 50 years, it has been strongly influenced by American culture, as well as largescale immigration from non-English-speaking countries. Again, both countries added significant cultural implications to the study and the findings can be generalised to other countries that are located in the developed part of the world.

Selection of locations for the fieldwork was limited by two important factors: time and cost. However, it did not necessarily affect the quality of information and credibility of the study because selected samples provided adequate quality and quantity of information to manifest the videoconferencing phenomenon from two perspectives (developing countries and developed 
countries) and on the example of both well established and less experienced centres on each side.

Table 4.1. List of DLC involved in the research

\begin{tabular}{|l|l|l|l|}
\hline DLC & Location & Host organisation & $\begin{array}{l}\text { Years in } \\
\text { GDLN }\end{array}$ \\
\hline $\begin{array}{l}\text { Mongolia } \\
\text { DLC }\end{array}$ & $\begin{array}{l}\text { Ulaanbaatar, } \\
\text { Mongolia }\end{array}$ & $\begin{array}{l}\text { World Bank Resident } \\
\text { Representative Office }\end{array}$ & $\begin{array}{l}\text { Since May } \\
2002\end{array}$ \\
\hline Moscow DLC & Moscow, Russia & $\begin{array}{l}\text { World Bank Resident } \\
\text { Representative Office }\end{array}$ & $\begin{array}{l}\text { Since June } \\
2004\end{array}$ \\
\hline DLC & Moscow, Russia & $\begin{array}{l}\text { Modern University for the } \\
\text { Humanities }\end{array}$ & Since 2004 \\
\hline $\begin{array}{l}\text { Australia } \\
\text { DLC }\end{array}$ & $\begin{array}{l}\text { Canberra, } \\
\text { Australia }\end{array}$ & $\begin{array}{l}\text { Australian National } \\
\text { University }\end{array}$ & Since 2001 \\
\hline $\begin{array}{l}\text { New Zealand } \\
\text { DLC }\end{array}$ & $\begin{array}{l}\text { Wellington, } \\
\text { New Zealand }\end{array}$ & $\begin{array}{l}\text { Victoria University of } \\
\text { Wellington }\end{array}$ & Since 2007 \\
\hline
\end{tabular}

\section{Access to Research Participants}

In order to gain access to the selected research sites and eventually to the interviewees, I initially approached the "gatekeepers" whom I happened to meet in person during my years of involvement in GDLN. I started by writing introductory letters to the managers of East Asia and Pacific (EAP) region that includes Australia, Mongolia and New Zealand as well as Europe and Central Asia (ECA) region where Russia belongs. Having received positive replies from both regional managers I proceeded by contacting individual DLC managers in selected countries. I personally knew the managers and training coordinators of DLCs in Ulaanbaatar, Canberra and Wellington so it was relatively easy for me to communicate with them. The ECA regional manager has kindly referred me to the DLC manager of the Moscow DLC who in his turn referred me to the contact people at the other two organisations in Moscow that are affiliated with GDLN. 
In my letter to the DLC managers I introduced the objectives of my study, requested the opportunity to visit their respective centres during my research fieldwork, observe videoconferences and interview some participants. All DLC managers replied positively and assured that they will refer me to many potential interviewees for my research from their extensive list of videoconference participants.

With commitment from four DLCs and two affiliates, I commenced my research fieldwork in Canberra where I interviewed nine people. In choosing the interview candidates I used a method called "triangulation of subjects" (Rubin, 2005) by trying to involve a diverse groups of GDLN clients of different genders, age groups, nationalities, cultures, educational and professional background and so on. This research has attempted as far as possible to avoid elite bias (Straus, Miles, \& Levesque, 2001).

During the Moscow leg of my fieldwork I also conducted nine interviews: DLC managers, participants of various videoconferences and one interpreter.

The third phase of my fieldwork took place in my hometown Ulaanbaatar, Mongolia. Again, nine interviews were conducted as planned. The respondents were selected from the extensive list of Mongolia DLC contacts - participants of various GDLN events most of whom I knew in person.

The primary data for this multiple case study came from observation of videoconferencing events and semi-structured interviews with participants of such events.

\section{Observation of Videoconferences}

The most organised DLC was that at the ANU, Australia: they had scheduled videoconferences for three months ahead with the topics determined and speakers identified. These were series of videoconferences entitled "Pacific Leaders' Virtual Forum" (PLVF) that have been initiated by the ANU. They came up with the idea, sourced funding from AUSAID and developed the first round of videoconferences in 2007. Government officials, civil society and business representatives from Papua New Guinea, Fiji, Vanuatu, Timor Leste, Australia and New Zealand discussed development issues affecting the region: 
Conflict and peace building in the Pacific Island States; Climate and fisheries management in the Pacific region; Infrastructure for growth: delivery and pricing; Anti-corruption policies; Entrepreneurship: advocacy and representation. Respondents in Australian and New Zealand cases often reflected on their participation in the videoconferences of PLVF series. I observed some of the sessions of PLVF in Canberra and Wellington legs of research fieldwork.

In Mongolia, I observed two videoconferences from the series of courses on Total Quality Management originated from the Asian Productivity Organisation and one videoconference which was a launching of a World Bank flagship publication “Doing Business 2007". In addition to observation of live videoconferences, I watched recordings of 3 videoconferences held in the Moscow DLC:

\section{Administration of Interviews}

Semi-structured interview is the most commonly used data gathering tool in qualitative research in information systems (Myers \& Newman, 2007). The primary data for this research was collected though interviewing participants of videoconferencing events. The average duration of interviews was 39 minutes with the longest interview lasting for one our and 13 minutes and the shortest one ending after just over 10 minutes. All conversations were digitally recorded for which the respondents consented before the interview.

The first nine interviews were conducted over the two-week period in July 2007 in Canberra whereas the interviewing nine people in Moscow was complete after ten days with the last interview taking place in August 2007. Commenced in the beginning of September, my Mongolian interviews were completed in one month. However, the Wellington-based interviewing process spanned through several months from late 2007 to early 2008.

In most of the interviews I was able to create an atmosphere of mutual trust and respect thus get candid responses to my questions. The greater majority of interviewees were relaxed and comfortable thus openly shared their experiences and perceptions with me. 
At the beginning of each interview, I gave the respondents a copy of brief introduction of my research along with the consent form which I asked them to sign. Interviewees also had a choice as to whether they wished to receive a summary of the research results when it is completed. No interviewee objected my using a digital voice recorder and they were assured that any information provided during the interview will be kept confidential be used only for research purposes. They all were pleased to know that the published results will not use their name, and that no opinions will be attributed to them in any identifiable way.

Interviews took place in different venues, mostly this was his or her own office or a meeting room in the respondent's organisation. In one case the interview took place in a café and in the other case in a park.

All interviews were semi-structured: I used beforehand prepared one-pager containing a set of broad questions regarding some of the factors that might affect the effectiveness of videoconferencing events (see Appendix). Using these questions just as a general guideline for our conversation I strived to encourage the participants to tell more of their personal experiences by intently listening and appropriately responding to the answers.

\section{Preparing transcripts and coding data}

On my return from the field trip, I began transcribing the interviews. The interviews were conducted in three languages: English, Mongolian and Russian. My fluency in all three languages enabled me to transcribe the interviews in original languages, indicating stalling words, pauses, hesitations, etc. It was a long and laborious task and it took several months to complete full transcription of all thirty-three interviews ranging in length from 20 minutes to over 1 hour.

The next step was to identify emerging themes. In order to do this, I used the NVivo software to simplify the process of extracting the recurring themes from the body of texts of transcribed interviews. For that, I entered the thirty-three word document files in three different languages into the NVivo program organising them in four country folders. Then, the first level of coding emerged 
from clustering the texts into the main topics in accordance with the research questions across all four cases. The second level of codes - also across all four cases - came forward from the recurring themes and important concepts. After I found, refined and elaborated the main concepts and themes, I systematically labelled them for easy reference. Sample tables of tree nodes and free nodes that composed the coding structure of this research is presented in Annex D. The coded material has been structured according to the purpose of my study which was to find answers to my research questions.

Then I sorted the coded data by grouping all texts with the same label into comparative tables (see Annex E) to see how the concept was seen overall, and then examined the country-specific data comparatively, looking for systematic differences and similarities between the countries on the same concept or theme. Two levels of comparative tables were created: firstly, I compared data from Russian and Mongolian cases - representing the developing countries, mostly recipients of content; and then I compared New Zealand and Australian cases - representing developed nations, usually originators and providers of content. Comparisons were made according to the classification of nodes that were derived from the research question such as cultural differences and emerging themes such as power relations. Coding method applied in this research has been informed by the book "Qualitative interviewing : the art of hearing data" (Rubin, 2005).

\section{7. Analysing coded data}

Once all data of my research coded in NVivo software has been structured, coded and compared, I thoroughly screened the material to see how it speaks to my research questions. I identified the most important ideas that shed light to understanding my research topic and analysed them in light of theoretical models that informed my research and themes suggested in the reviewed literature. The concepts and themes frequently mentioned and indirectly implied by interviewees and those that emerged from comparing the country cases, or from stories told or metaphors used by the interviewees in turn suggested new and related concepts and themes (Rubin, 2005). Then I went 
back to my data and looked if and how these newly emerged themes and concepts were supported elsewhere in the interviews conducted in four countries of my multiple-case research and whether these were backed by the observation notes. If the concept or theme was not supported in at least two cases, I dropped them for lack of evidence or merged some thus refined the emergent themes. In the next step in my analysis I tried to process the meaning and define the new concepts that explain my research problem.

While writing up the country cases I aimed at reporting on important concepts and themes using the same structure: a) Cultural differences of participants; $b$ ) Language differences and translation issues; c) Facilitation and moderation issues; and d) Presentation issues. Having reported on all four cases, I already had a set of the most important factors and themes and then I examined them across all four cases. I also examined whether these findings were supported by sources other than interviews - observation notes and other documents.

In writing up the discussions chapter I focused more on those themes and concepts that were contributing to the exploration of my research problem. I gave more emphasis to those factors and concepts that were supported by triangulated data. These themes were synthesised from all thirty-three interview transcripts and notes taken during observation of nine live and three recorded videoconferences.

Finally, I looked into my data from the perspective of concepts and themes suggested in the literature. For example, the references talking about power relations were analysed in light of Hofstede's (2001) power distance dimension of culture and in relation to other studies on the topic. Other themes such as the concept of 'videoconferencing culture' was an original concept that emerged from data collected in this research. A theme about the impact of the videoconferencing technology on the participants' perceptions of cultural factors was suggested by references classified under cultural differences, presentation issues, technology apprehension and other nodes throughout the four cases. 


\section{8. The role of the Researcher}

Interpretive researchers are attempting to understand phenomena through accessing the meaning the participants assign to them (Orlikowski \& Baroudi, 1991). Interpretive researchers should have a view of their own role in this complex human process and select one out of two identified roles: the outside observer and the involved researcher, through participant observation or action research (Walsham, 1995).

In this multiple case study, my role as a researcher would be that of the outside observer. In this approach the researcher is seen as not having a direct personal stake in various interpretations and outcomes, and thus the interviewees are relatively frank in expressing their views (Walsham, 1995). Hopefully, my personal communication skills and ability to establish a relationship of trust with the respondents will greatly contribute to the quality and usefulness of data that can be obtained from the interviews.

The majority of GDLN videoconferences are one-off events comprising of a single 2-hour session. Normally, the participants gathered for a single-session event meet for the first time in the videoconferencing room unless they've known each other before. Thus, they have no opportunity to build up a group culture during such a short collaboration nor they have much confidential information to share. Therefore, the main disadvantages of the outside observer's role - namely that of the researcher not being a member of the organisation and lacking an insider's viewpoint, and that of the researcher being debarred from access to confidential or sensitive information (Walsham, 1995) - are not applicable in this research.

However, the role of a 'neutral' observer does not mean unbiased because we are all biased by our own background, knowledge and prejudices to see things in certain ways and not others (Walsham, 2006). 


\section{9. Biases and Limitations}

Researcher bias has more room to creep into the case study compared to other research methods because it involves more interaction between the researcher and the subjects than most other research methods (Walsham, 1995).

Two types of bias may be introduced by the researcher during the collection and analysis of case data: the effects of the researcher on events and the behaviour of participants at the case study site, and the researcher's own beliefs, values and prior assumptions which may prevent adequate investigation and consideration of possible contradictory data and unnecessarily influence the analysis of the case study evidence (Darke, Shanks, \& Broadbent, 1998).

Other bias that has to be addressed is related to the communication problems between interviewer and interviewee particularly when the language used in the study is not the native language of the interviewer/interviewee (Karahanna, Evaristo, \& Srite, 2002). Probably, this is more a limitation than a bias. English is my third language after Mongolian and Russian but I'm confident that I was able to effectively communicate with my interviewees in Australia and NZ in English and even more so in dealing with Moscow-based respondents in Russian language.

According to Walsham (2006), 'neutral observer' means that the people in the field situation do not perceive the researcher as being aligned with a particular individual or group within the organization, or being concerned with making money (as consultants are for example), or having strong prior views of specific people, systems or processes based on previous work in the organization.

Certainly, I was not doing this research for money but I needed to acknowledge my possessing a number of researcher's biases mentioned above that needs to be addressed and reported for.

- Three years of personal experience as a training coordinator for Mongolia DLC could have been a source of bias in treating the DLC in Ulaanbaatar differently than other sites. On the other hand, my prior experience with the network helped me to understand the phenomena thus this bias brought 
more 'good' than 'harm' to the research. As an interpretive researcher, I needed to continually question my own 'prejudices' in order to succeed in developing a comprehensive understanding about the phenomena under the study (Prasad, 2002).

- The Mongolia DLC is co-located in the premises of the World Bank office in Ulaanbaatar and in fact I was a World Bank staff. My employment with this international development agency had a strong influence in terms of corporate culture. Therefore, I avoided looking into the communication under the study through the World Bank lenses and was duly following the case study protocol in order to maintain a broad perspective in my research. The case study protocol is discussed in greater details in Section 3.5.

- Understandably, I had an extensive circle of personal contacts in Mongolia, many of which occasionally participate in various videoconferencing events. There was a high probability for the chance that some people whom I know well will participate in videoconferencing events under this study and agreed to be interviewed. Again, I made efforts not to differentiate them from the rest of respondents but benefited from such occasions.

Cultural values, attitudes, and behaviours affect a researcher's understanding, processing, and analysis of data and information, therefore it is reasonable to suspect that researchers may be producing culturally biased research, particularly in research relating to global IS, cross-cultural research, and any research that involves multicultural research populations (Pauleen, Evaristo, Davison, \& Ang, 2006). Personally, I believe that the impact of cultural bias on my research was minimal given my extensive multicultural experience confirmed by the following facts: I was a member of multicultural teams working on various development projects (almost 4 years); I was employed by the World Bank (3 years) and exposed to its strong corporate culture that highly values cultural differences; speak 3 languages; experienced life in different cultures (Moscow, Russia - 4 years; Maastricht, the Netherlands - 1 year, Wellington, New Zealand - 4 years) and visited over 25 countries; have friends and colleagues in even more counties. 
In working on this research, I reviewed papers analysing data collected not only in North American and European context but also in the contexts of developing countries and economies in transition; focused my research on cultural affiliation and social identity of individuals; paid attention to communication problems inherent in interaction that involves different languages and discussed my topic with international friends and colleagues.

However, I have to admit that the choice of my research topic was not free of cultural bias of some sort: I assumed that focusing my study on a global videoconferencing network will have more appeal to the intended audience because I doubted that Mongolian context will have high relevance or applicability for international research and practice. Nevertheless, I fully agreed with the following comment:

"IS journals regularly publish articles that are situated in the context of a single nation state, with little or no attempt to explain why or how the findings are relevant beyond the borders of that state, let alone globally" (Pauleen, Evaristo, Davison, \& Ang, 2006`, page 358)

This research is conducted in accordance with generic ethical practices, as well as in conformity with the requirements of the Human Ethics Committee of Victoria University of Wellington. Approval was obtained from the latter committee on July $4^{\text {th }}, 2007$ in accordance with the statutes of the university, prior to the commencement of the field work. In accordance with the requirements, all interaction with interviewees was conditional upon fully informed written consent.

There were certain limitations to the proposed research that need to be addressed.

- In examining the nature of cross-cultural communication that occurs via room-based videoconferencing technology, assumption was made that events under the study will be free of technical problems. However, observations of actual videoconferences during the data collection period proved it otherwise thus I included this aspect into the scope of my research. 
- Preceding the actual videoconference is thorough preparatory work that involves communication between the parties via e-mail and sometimes by telephone. It was possible that respondent's perception about the effectiveness of GDLN event may not be free of influences from the earlier communication that occurred via channels, other than video.

- It was difficult to generalise the study findings throughout the GDLN although it might be relevant to some DLCs in Central Asia, East Asia and Pacific regions of GDLN.

\section{10. Issues of Validity and Credibility}

The ontological, epistemological, and methodological choices made during design, data analysis, or data representation stages affect the study and its validity in different ways (Koro-Ljungberg, 2004). The strength of analysis in interpretive studies derives from the strength of the explanation of the phenomena based on the interpretation of data (Darke, Shanks, \& Broadbent, 1998). In order to establish credibility to the reader the researcher must describe in detail how the research results were arrived at, and to establish validity in the view of the reader the researcher must present a coherent, persuasively argued point of view (Walsham, 1995).

Golden-Biddle and Locke (1999) proposed three dimensions for convincing the reader that the knowledge or a 'finding' of the study is worth paying attention to: authenticity, plausibility and criticality.

Dimension of authenticity focuses on the setting studied and concerns the ability of the text to show that the authors have 'been there', and were genuine to the experience in writing up the accounts. In comparison, the dimension of plausibility focuses on the reader and suggests that in order for a work to convince, it must establish connection between the subject matter and the reader's knowledge and experience. Criticality also focuses on the reader: it concerns the way in which the text provokes readers to re-examine their takenfor-granted ideas and beliefs.

The quality and credibility of qualitative analysis can be enhanced by dealing with three distinct but related inquiry concerns (Patton, 1999): 1) rigorous 
techniques and methods for gathering, coding and analyzing qualitative data, for example use of triangulation of data sources; 2) the credibility, competence, and perceived trustworthiness of the qualitative researcher; and 3) philosophical beliefs about the rationale for and appreciation of naturalistic inquiry, qualitative methods, inductive analysis, purposeful sampling, and holistic thinking.

Recognising, addressing and reporting potential sources of researcher bias, including initial expectations of study results, strengthens the credibility of qualitative report (Drisko, 1997). In Section 3. 9, I acknowledged the researcher biases and explained how these are addressed in this research.

Based on the assessment of the proposed study in relation to above concerns I have sufficient arguments to believe that the intended audience - academics and practitioners - will accept the knowledge resulted from my research as worth their attention.

\section{11. Chapter summary}

I have chosen an interpretive case study approach for my research in order to understand perceptions of individuals who participate in videoconferencing events. This is an exploratory study because it appears that the literature is relatively silent when three topic areas intersect: cross-cultural communication, video-mediated communication and development communication. The phenomena of interest will be investigated in four different locations therefore I adopted a multiple-case embedded design for my study in order to seek for cross-case patterns in perceptions of people attending videoconferencing events. Use of a case study protocol will guide me in carrying out the data collection, keep me focused on the subject and effectively manage resources and time in the field.

This chapter also described the process by which I selected research sites, observed videoconferencing events, gained access to individuals and conducted interviews that subsequently supplied the primary data for this research. It also guides the reader through the process of data processing and analysis. 
In this inquiry my role as a researcher would be that of the outside observer. The source of data consisted of interviews with participants and observations of videoconferences, complemented by relevant documents. This data was analysed at three levels: within the case, across pair of cases and across all four cases. Comparison of findings and search for patterns took place at all three levels of data analysis.

Having identified potential biases and acknowledged the limitations, I am determined to gain in-depth understanding of cross-cultural videoconferencing phenomena in the context of development. The Chapter concludes by addressing the issues of validity and credibility in interpretive qualitative study. 


\section{Chapter 4: Data Interpretation and Preliminary Case Analysis}

This chapter aims to introduce readers to the data interpretation and contains four sections: each sub-chapter is devoted to a separate case study. Data gathered from individual cases is presented in chronological order, in a format that is consistent throughout this chapter and Chapter 5 - Discussion.

The themes within each case are presented in the following order: Cultural differences of participants; Language differences and Translation issues; Facilitation and Moderation issues; Presentation issues.

To help the reader to better understand the discussions regarding Cultural differences of participants, it is appropriate to re-emphasise that the term Cultural differences takes account of differences at a variety of levels of culture including national, ethnic/racial, various sociological subgroups categorised by gender, social class and professional affiliation (Y. Kim, 1984).

\section{1. The Australian Case}

The fieldwork for the Australia case was carried out in July, 2007 at the GDLNAustralia hosted by the Australian National University in Canberra. The data gathered includes interviews with nine participants of videoconferences, presenters and people working for GDLN-Australia as well as observation notes from three videoconferences that I attended. Additional resources include a video recording of one of the observed videoconferences and other documents about GDLN-Australia.

Respondents in the Australian case consisted of Australians, including those immigrated from the United States and United Kingdom as well as representatives from Vietnam, Indonesia and Timor Leste who were studying

in Australia at the time. The group comprised faculty members, graduate students, government officials - men and women aged from 25 to 55. They participated in videoconferences with different roles: some facilitated the events, some moderated the sessions as well as made presentations whereas others attended videoconferences as regular participants. 


\section{Cultural differences of participants}

Interviewees in the Australian case discussed in a positive light the effects of cultural differences of participants on the effectiveness of videoconferences and viewed the diversity as an opportunity, not an obstacle.

Some participants believed that videoconferencing technology facilitated effective cross-cultural communication and diminished cultural barriers among the participants.

"I think it's a really good way of breaking down the barriers that culture could otherwise put up. You know, because there's good will when people come in ..." (Respondent 2)

"It enriches the quality of that dialogue ... when people from other backgrounds, different religions and everything, talk together. It's just like sort of sharing ideas and it affects a lot of people" (Respondent 7)

"I think it's a great opportunity to get a whole lot of cultures together talking together and I think that it's actually an advantage not a disadvantage and that these people are able to all share, you know, a common knowledge, common grounds" (Respondent 1)

According to the above quotes, interviewees believed that the cultural diversity of the participants was an advantage that enriched communication and minimised the differences of cultural backgrounds.

However, some respondents believe that when communication occurs via technology, not face-to-face, people engage in communication without being concerned about cultural differences between one another. In other words, communication takes place in a culture-free virtual zone where differences are 'masked' or switched off.

"I think the technology tends to mask the cultural differences a little bit because people are slightly apprehensive about it and they respect it and they're not quite sure about it so they are little worried that it [videoconferencing technology] takes their attention a little bit away from worrying about the person on the other side" (Respondent 2) 
"In some ways it is also an un-cultural place ... you feel that people are quite chatty about things in a sense ... like they will be less forthright and talkative if it was entirely within the country ... So it's an odd kind of transnational space that everybody is operating in." (Respondent 6)

The context of the interview suggests that the phrase 'worrying about the person on the other side' used by Respondent 2 refers to the cultural differences of participants of videoconferences.

The last quote above by Respondent 6 also implies that the videoconferencing setting enables participants to openly discuss sensitive or challenging issues that they would feel uncomfortable talking about in their local environment. The following quote by an Australian interviewee - who has worked with Timorese people for many years - echoes this idea:

"We had Timor Leste come in and they were discussing corruption with the rest of the region. And that to them was culturally not acceptable to really discuss this, so they won't talk about it but they have problems with it. But then they saw the way the other countries are handling it and that affected the way ... now they are only too happy to be involved" (Respondent 2)

Some respondents believe that cultural differences of participants do not influence the effectiveness of communication that takes place during videoconferences

"In the actual bit where the group, the five different groups was hooking together, I didn't see any cultural problems at all. (Respondent 9)

In terms of cultural differences, I don't think that affects much. Because nowadays people are globalised, so they ... that's not the first time they met with people from another country" (Respondent 8)

The 'time is money' concept is relevant to videoconferencing because videosessions are structured minute by minute, have a precise running order and predefined floor-taking arrangements. Cultural differences among the participants are reduced by the formality inherent in videoconferencing. All 
participants have to obey the "videoconference culture" and leave aside their cultural differences.

"I think that because it's done in a way that most conversations are carried on, they're done in a formal sort of way, so people actually have to behave themselves... That's a nice way of sort of removing yourself a little bit but still being involved. I think it's really working well." (Respondent 2)

The formality and strict timekeeping seen in videoconferencing is congruent with the dimension of time in Hall's (1976) cultural model. Different cultures have different perceptions of time and these perceptions have important implications for cross-cultural communication. However, in communication that takes place via videoconferencing technology, participants from a polychronic culture (Hall, 1976) need to consider that the time is limited:

"For Pacific Islanders, it takes a little bit of time ... to relax and open up, it's kind of, you can't just go in and have this business conversation ... you need to have a conversation rhythm, and start off slowly ... that's kind of a limitation for me. You can't really do that [during videoconferences]...because of limited time" (Respondent 5)

The above quote suggests that people from polychronic cultures that view time as flexible (Hall, 1959) might feel disempowered because videoconference time is very limited. Although they might have something to say, they decide not to during a videoconference. However, that might be the case not only due to cultural aspects but because of the individual's personality.

"Some people want to just express their ideas right away, some may want to hold the idea, think more about it before speaking out" (Respondent 8)

Though, one of the respondents noted that speaking in public is difficult no matter what culture one is coming from:

"Asking question is a painful process, nobody wants to be the first one to talk. I think that's not really a Pacific Islander thing..." (Respondent 5)

Power relations between the participants at a particular location have significant implications on the effectiveness for cross-cultural communication in 
terms of openness of discussion and trustworthiness of the statements being made. Some people might feel obliged to express opinions that fit the viewpoint of their more senior colleagues - a result of the subordinate's fear of disagreeing with superiors (Hofstede, 2001). The following quote by Respondent 6 illustrated such a situation in one culture and contrasted it with another culture based on personal experience:

"Once when the Fiji lot was settling down and suddenly the minister appeared ... so, everybody was rapidly tailoring their opinions to what they were going to say.... In PNG people tend to be a bit less sort of deferential to each other and more prepared to speak their mind whatever level they are." (Respondent 6)

Two other respondents discussed the issue of power as well, but at different levels: differences of values and attitudes among various organisations in one country and commercial/business interests of different countries in a particular industry.

"[In Fiji] there is usually a bit of a tension between the NGOs and the government" (Respondent 6)

"I can't think the culture matters here. There are severe differences among these groups in terms of their stakes in the fishery... It's hard for them to be too cooperative and too interactive because they actually compete quite a bit." (Respondent 4)

Information provided above facilitates an understanding of participants' viewpoints towards the communication. The data showed that most respondents think about interaction during videoconferences as just normal communication, not cross-cultural, because they live and work in a multicultural society. In other words, their everyday communication is culturesensitive.

"I direct a program with students from over 30-40 countries, so I'm already very sensitive to cultural differences anyway." (Respondent 4) 
"The main presenters were so used to dealing with presenting lectures to groups of people that come from all different sorts of cultures so that's quite a common thing in this school" (Respondent 5)

Interviewees' backgrounds reveal that most of them have been exposed to many different cultures - national and organisational - that reflect the multiple, complex forces that shape their beliefs and behaviour (Gallivan \& Srite, 2005).

"I'm born in America, taught in Canada, live in Australia, ... married to [laughs] all kinds of people ... so, yeah, it's easy for me (Respondent 4)

I joined the WB in Timor... And now I'm working part-time ...here in Canberra." (Respondent 7)

"I was working in the Vietnamese government. Then I came here to study and so I joined the GDLN-Australia working part-time." (Respondent 8)

"I'm Australian ... I've done quite a bit of work at the United Nations ... I lived in China for the last year ... my girlfriend is Chinese" (Respondent 9) The repeating ideas expressed by respondents in the Australian case can be summarised as follows: videoconferencing technology usually does not present barriers to cross-cultural communication although the limited time of videoconferences might restrict some participants' engagement, especially those coming from polychronic cultures. Nevertheless, the majority of participants set aside their cultural differences and adhere to the formality of "videoconference culture". Interviewees reported illustrations of power relations at different levels that they observed at some participating sites. In general, each respondent contains a complex set of 'cultural identity and experience layers', some to the extent that they do not consider the communication that takes place during videoconferences to be cross-cultural.

\section{Language differences and translation issues}

All three videoconferences observed in Australia involved five participating sites: Timor Leste (GLDN Centre, Dili), Fiji (University of South Pacific, Suva), Papua New Guinea (GDLN centre, Port Moresby), New Zealand (Victoria University of Wellington, Wellington) and Australia (Australian National 
University, Canberra). The Timor Leste site was the only one using simultaneous interpretation during the three sessions observed. Official languages in Timor-Leste are Tetum and Portuguese whereas English is the first official language in Fiji and Papua New Guinea. Participants at all other sites were Anglophones and did not use translation services.

Most Anglophone participants as well as some non-English speakers regarded language differences as a barrier that could be successfully overcome because they assumed that GDLN sessions were adequately translated. This assumption cannot be confirmed in the Australian case because the interviewees were either native English speakers or proficient English speakers who heard the simultaneously translated version of their foreign counterparts' speaking. In addition to language barriers, participants at various sites had differing levels of knowledge on the topic that might have impacted the overall communication effectiveness, according to some informants:

"The only issue [of] language was [with] the East Timorese. They had a translator, and I think that probably worked. The translator was fine because the questions they were asking were not the questions indicated that they misheard. They were questions that they didn't understand, because their level of knowledge was so far behind the other peoples'." (Respondent 5)

"If we have a good interpreter, with good knowledge about the topic, then it can be easily overcome." (Respondent 8)

The assumption of regular participants that most videoconferences were adequately translated was challenged by the interviewees who facilitated and moderated videoconferences. They admitted that the quality of translation was not always satisfactory and fully acknowledge that finding skilled interpreters was difficult.

"I know from first hand experience ... that often, even with the most capable of the interpreters, you never really [pause], well you very rarely completely, fully understand the specific meaning of what the other person is trying to say." (Respondent 9) 
“Of course you get a little bit 'lost in translation' but I don't think you get that an awful lot." (Respondent 2)

"I think that's something which they've been working on over the time and it's not always easy to get a good translator who can really translate well." (Respondent 1)

“On these, sort of, local networks [meaning GDLN], I'm not sure if it works very well. It's very hard to simultaneously interpret as you know... And, depending on that [chuckles] interpreter, you know, it may not be as effective as you wanted to be." (Respondent 4)

Non-English speaking respondents believe that the translation issue is a serious problem in cross-cultural communication and emphasized the scarcity of skilled simultaneous interpreters in their respective countries. Some of them pointed out that this problem was linked with funding issues.

"This is the most important issue faced by the organisers, our colleagues in Timor right now... Most of the time Timor Leste uses simultaneous interpreters and it's really, really hard sometimes to understand them ... All the interpreters are freelancers. So when we call them, and they have something else to do, they cannot come ... If you want a very good quality of translation, I think you also have to spend the money." (Respondent 7)

"At the events, interpretation and translation is important, but I think it can be easily overcome. Oh yes. And also, we need funding for that." (Respondent 9)

Simultaneous interpreters work under high stress conditions: the constant information load, the time factor, the tremendous amount of concentration required, fatigue, the confined environment of the booth, etc. (Kurz, 2003, p. 51). Moreover, the work of translators becomes even harder when presenters talk too fast and use professional terms. Respondents agreed that speakers should simplify their language to facilitate easier translation. However, the idea of using less complicated language where possible, or of the need to translate specialist terms, is more likely to be associated with the spectre of lowering standards (Haggis, 2006, p. 529). However, the interviewees do not comment 
whether presenters' using simplified terms or in other words, 'dumbing down' (Dunlop, 2003) what they say had any impact on the overall effectiveness of multi-lingual video-mediated communication.

“Obviously you have to talk a little bit slower, maybe the language has to be modified slightly so it's easier for the translators to translate... I think simple is better in this sort of environment." (Respondent 1)

"You do have requests in say places like Timor-Leste, where people would ask the presenter to use simple words so that the translators won't get lost and I think that's very valid and presenters usually try." (Respondent 2)

At the same time, the quotes above may also suggest that simplifying the communication vocabulary in order to ease the translator's task could potentially have an impact on the effectiveness of communication.

\footnotetext{
"The problem is the usual one, you know, complaining that I'm speaking too fast and you know, the mechanics of translation not working." (Respondent 6)
}

"I've been [hearing] a lot of complains from participants in Timor, asking the participants to talk slowly because it's hard for the interpreter to catch what people are saying from other sites and be able to translate it... and that's becoming ineffective communication." (Respondent 7)

Some respondents claimed that the interpreter's knowledge on the topic was crucial for more effective translation.

"On a topic like this [fisheries management] it would be a nightmare. The interpreter will have to know more or less what I know, right?" (Respondent 4)

"Quite often you are dealing with complex concepts and that can be a little bit difficult but then the presenter... would explain what they mean so that really helps." (Respondent 2)

Many respondents perceived translation-mediated communication as absolutely normal so that sometimes they didn't even notice that the communication was translation-mediated. That was mainly because the 
respondents lived in multilingual societies and engaged in cross-cultural communication in their daily work:

"In my work, I deal with people from all over the world all the time and interpreting and stuff, it's just totally natural for me." (Respondent 6)

“And it's just perfectly normal. I don't even really notice." (Respondent 9)

"When it comes to sort of content or meetings... I mean, they're up to speak like we are. And that doesn't bother me." (Respondent 4)

One of interviewees believed that people generally adopt a positive attitude when they engage in cross-cultural communication. Friendliness and willingness to understand one another reduce communication barriers such as language differences:

"I'm trying to understand the kind of cultural thought processes they have and I'll try to understand what they are trying to say in that context rather than just expecting them to be exactly like me." (Respondent 9)

This corresponds with another respondent's observation on page 98 that people come to GDLN events with 'good will'.

To conclude, participants of multi-site translation-mediated videoconferences believed that language barriers can be overcome by quality translation. However, they agreed that the precise translation of a meaning from one language to another is practically impossible - something does get 'lost in translation'. Videoconference organisers talked about a scarcity of experienced simultaneous interpreters and the complexity of a translator's work. The panellists facilitate more accurate translation and contribute to the effectiveness of communication by clear pronunciation, using simple words and maintaining an adequate pace of speech. Sometimes, people failed to notice that the interaction was facilitated by translation because they exist in a multicultural society and regularly engage in cross-cultural communication at work and in everyday life. 


\section{Facilitation and moderation issues}

Role descriptions for the site facilitator and session moderator in a GDLN context have been discussed in detail in the Introduction. However, the interview responses revealed that people involved in organising or chairing videoconferences understood the difference between two functions whereas regular participants perceived that the roles of facilitator and moderator were fairly similar and interchangeable.

"Moderator or facilitator - they have very similar roles - but the bottom thing is to keep people motivated. To keep people engaged in the conversation." (Respondent 8)

"I tend to think of a moderator being more in the area where programs are being delivered and the facilitator looking after the areas where the programs are being received. It's quite a similar sort of function in a way but there is a just a little bit of a distinction." (Respondent 2)

"It was mostly just calling for questions, [] Facilitating in that way." (Respondent 4)

Regular participants and videoconference organisers alike agreed that site facilitators play an important role in making the videoconference possible by performing multiple tasks before, during and after the sessions. Most respondents were convinced that individuals with extrovert personality who possessed excellent communication and organisational skills could effectively facilitate and/or moderate video-sessions by enabling effective interaction among the participants.

"It's a matter of liaising very well with all the centres. Communicating is extremely important in this job. The facilitator has to be someone who can engage people... you have to have dynamic people in order to really engage your audience." (Respondent 1 )

"You need someone who is dynamic and interactive, and who can get people talking... It can be quite a skill... difficult to do sometimes." (Respondent 5) 
"I think a facilitator is very important because the facilitator will help overcome a lot of difficulties in communication. Sometimes they [participants] may feel bored, discouraged, reluctant, afraid sometimes to communicate. A facilitator will help participants overcome those." (Respondent 8)

Perhaps, the interviewees referred to the moderator's role when they commented that only a very enthusiastic and resourceful individual was able to ignite the participants and promote a lively and productive discussion.

Respondents who facilitate videoconferences revealed that recruiting participants to a videoconference required lots of effort: identifying potential participants, communicating with them and effectively promoting the event and finally, persuading them to come to the session.

"If you ask them to pay to come - very difficult! Even if you offer free - it's not easy! If you offer some incentive for them - it's easier. So, [laughs] recruiting people is tough." (Respondent 8)

One of the respondents acknowledged that signing up participants for GDLN events was easier in a developing country than in a developed country. Based on my personal experience of promoting and facilitating GDLN events in Mongolia and New Zealand, I fully agree with this statement.

"In Timor, accessing people is easier. It's easy for me to go and talk to people in high level ... I can just go and talk to them "oh, we have this program, ... would you like to come?" and then they say, "yes"... While here [in Australia], you know, it's a bit hard to go and talk to them: you have to make appointment, etc." (Respondent 7)

According to Respondent 1, the role of facilitator at GDLN-Australia comprises multiple complex functions because this DLC not only recruits participants and delivers videoconferences but actually initiates, sources funding and develops programs for the EAP region. The DLC manager and presenters recognize that facilitators work hard to make an event possible. 
“It's critical. Without [a facilitator] you can't have a program." (Respondent 2)

"I know a lot of preparatory work has been done by the local GDLN place to get that particular group of people there and sell it to that group of people." (Respondent 6)

When the skills and efforts of the facilitator result in a smooth and productive interaction, the participants practically do not pay attention to how the communication was made possible; however, they become aware of the facilitator in cases when something goes wrong. (Hedestig \& Kaptelinin, 2005). Observations of video-sessions in the Australian case proved that the facilitator unobtrusively monitored the videoconference process yet immediately stepped in when a problem occurred: contacted the $\mathrm{NOC}^{1}$ if any of the participating sites dropped out of the session, informed fellow facilitators at other sites if they forget to mute the microphone, reminded the presenters to slow down when necessary, controlled the camera, adjusted the room temperature, facilitated activities during session breaks and so on.

"What we've learnt over time is that when things do go wrong you always have a backup... if we lost all the groups ... because of the technology, we'll make sure you have a local facilitator who ... can engage in ... so that ... we haven't lost the people, we haven't lost the whole momentum of the whole videoconference, until the technology can be fixed, we already have a backup plan." (Respondent 1 )

It was noted however that facilitators at participating sites demonstrated varying degrees of competence and resourcefulness. For example, a site facilitator in Australia and Fiji controlled the camera well by zooming in to the speakers and zooming out to the participants when nobody spoke from their end. They have efficiently handled minor technical problems such as the cut off and loss of audio. At the same time, cameras at other sites were mostly static, showing the entire room at all times. The site facilitator in PNG had problems with managing the microphones often forgetting to mute them when speakers

\footnotetext{
${ }^{1}$ Network Operations Centre
} 
finished talking. Perhaps the site facilitators had different levels of videoconferencing experience, technical, communication and interpersonal skills.

According to Havens (2001), the role of the moderator is to ensure that the speakers remain focused on the given topic and address all key points; maintain the pace and continuity of the program; ensure that all panelists participate in the discussion; and ensure that the program stays on time.

Reflecting on his one-off experience of videoconference, one of the regular participants thought that the moderators succeeded in engaging participants in the discussion while following the agenda and keeping track of time.

"I thought that was pretty effective from the point of view of what I think he [moderator] was trying to achieve from that meeting, which was to keep it pretty structured because [there was] a lot of material to cover in two hours..., but to enable participants to think up what they want to say and be really active." (Respondent 9)

The Observation notes contain an illustration of a moderator's floor management and timekeeping skills. Granted the floor, a participant at one of the remote sites made an apt comment and continued on, encouraged by people's attention. The moderator managed to interrupt him firmly yet tactfully by saying "Right, what you're telling us is a great example of..." thus preventing the discussion from going off the track.

On the other hand, the moderators themselves admitted the challenges they faced - managing the time, following the predetermined structure - and shared how they dealt in particular situations.

"I have tried to make my talk as short as possible but I could say it wasn't that successful, I mean people have said some really interesting things, and it was really frustrating that we couldn't get enough time to talk." (Respondent 5)

"I thought we ought to try and deal with concepts in literature more and try and focus the discussion a bit more on particular sectors... But its difficult to 
do it in that framework really, with a changing population in each session ... so [I] pulled back a bit on the framework and the didactic stuff and just treated it as a conversation people enjoy having..." (Respondent 6)

The above comment was related to the fact that a moderator had to give up the initially planned structure of the videoconference series and take a different, less formal approach. The plan was to base the discussions on the reading materials that were sent out ahead of the session. The videoconference organisers - university lecturers - assumed that the participants would come to the sessions prepared e.g. having read the papers in advance. However, it appeared that the majority of participants just showed up to the videoconference without any preparation. Some might have been unaware that they were expected to prepare. Therefore, the moderator had to improvise and encourage discussion by briefly introducing the concept, giving time for site discussions and then wrapping up. One of participants liked the approach and praised the moderator for his ability to stick to the timeframe:

"I thought the way he did it was good, throwing to each side each time, to make sure everyone had a chance to say what they wanted to say, but at the same time, [when] somebody started to talk a bit too much, he would just say, "Oh ok, well we need to wrap it up..." I thought it was quite good, the way he did that." (Respondent 9)

To sum up, the roles of facilitator and moderator have a lot in common and are equally important for videoconference outcome. Moderators chair the discussion during the videoconference whereas the facilitator's functions extend beyond the actual event and include a number of preparatory tasks as well as session follow-up activities. Both roles require excellent communication, time management and people skills. In addition, the facilitator should possess technical troubleshooting skills and have a back-up plan in case things go wrong. Data suggests that moderating a video-session is not an easy task, yet one should be capable enough to adjust to the situation while keeping the audience in control. 


\section{Presentation issues}

According to Havens (2001), being a panellist in a videoconferencing setting requires mental agility and the ability to be a "team player," whereas not all great lecturers have these skills. An interviewee's remark fully corresponds with this statement:

"Many academics are used to getting up in front of a group of people and lecturing. So, there is no interaction... whereas GDLN or those videoconferences are much more interactive and they should be." (Respondent 4)

One of organisers was positive that if the presenter turns into a 'talking head', the session will fail because in the videoconferencing setting people get bored faster than in a face-to-face environment.

“If the speakers know the subject but ... don't have presentation skills, they may run the power points all the time. The power points [can be] too small, participants can't see, they don't see the speaker, [they] just see the power point. And the speaker just talks all the time and participants [start] falling asleep because they ... can't stand just continuing through the videoconference. People get tired, bored very quickly. Much more quickly than in a face-to-face [setting]." (Respondent 8)

Clarity of speech is especially important in cross-cultural translation-mediated communication. Discussing issues of simultaneous interpretation, Kahane (2000) mentioned the speed of speeches, non-native accents, private jokes, etc. among other trials regularly faced by interpreters.

"Presenting well, presenting clearly is very important in the same way that its important in a face to face lecture, probably, just as important if not more important [in] this context, because you have to reach through the medium and the camera, ... and you might have a delay in sound or whatever, so yes its very important." (Respondent 1 )

Transmission delay mentioned by the above respondent, creates the impression of long pauses before the arrival of replies from remote participants, which in turn creates uncertainty and produces the wrong signals (Braun, 2007). For 
example, such hesitations were usually interpreted as an inability to produce an appropriate reply or as a disagreement (Clark, 1996).

"When you deal with participants, like ... from Timor, it is very important for the presenters to understand the situation and be able to engage the participants by stressing the idea and in this case, talk slowly." (Respondent 7)

One of the DLC support staff stressed the significance of speakers' communication and presentation skills as well as their ability to use the language that is most suitable to the intended audience.

"[It is important that the speakers] not only know about the knowledge, but also [they] have to speak well... [we] have to make sure that the topics will be suitable for participants, [match] their interest, [be] at their understanding level, not too difficult, not too easy." (Respondent 8)

Videoconference organisers were certain that the speakers have to adjust their way of presenting in order to be successful in a videoconferencing setting: avoiding long speeches, limiting the use of power point slides, encouraging interaction rather than focussing on the delivery of material.

"It's also important that the presenter understands the technology, and adjusts his presentation to suit the technology. The technology doesn't adjust very well to long monologues. That becomes quite boring ... But if you want to have a really good GDLN session ... when people ... talk across cultures ..., you need to break your conversation up into smaller bits so you can have regular interaction." (Respondent 2)

"It really depends: if it is a training program, obviously they have got to get across ... what they are talking about. [But if] it's ...like a sharing of knowledge ...you want to engage each of the countries,... you don't want the presenter to do a lot of presenting. I try and say to our presenters... avoid having PowerPoint's." (Respondent 1) 
Two university lecturers interviewed often get invited to make presentations during videoconferences. Both admitted that their usual lecturing style might not work well in videoconferencing.

“Don't do much formal lecturing ... I don't think that would work at all...Well, it might work very well in videoconferencing but I can't imagine given the difficulty of the technique anyway that it would engage people for very long." (Respondent 6)

"I think videoconferencing works well when you have a dialogue or a discussion among groups of people. It doesn't work as well when you're trying to teach some information like you would in a classroom. It's a different vehicle." (Respondent 4)

In a videoconferencing setting the speaker's mobility is limited because they need to remain within the camera angles, whereas many lecturers got used to the freedom of space and movement, walking around, writing on the board, etc. The first quote belongs to a respondent who was a former student of the presenter and the second quote belongs to the presenter himself.

"When they teach in a classroom, they walk up and down the room and wave their hands and [do] all the things that Australians and Americans do." (Respondent 5)

“Normally I move around a bit more... absence of something to write a word on, or something [like] a whiteboard is an issue. But being pinned on doesn't, didn't help." (Respondent 6)

It is evident from the lecturer's response that during the videoconference he was not as comfortable as he would normally feel in a university lecture hall.

Nevertheless, as one of the regular participants observed, the presenter succeeded in delivering new material and, at the same time, keeping people engaged and motivated.

"I thought that was pretty effective from the point of view of what I think he was trying to achieve from that meeting, which was to keep it pretty structured because [there was] a lot of material to cover in two hours, ... but 
to enable participants to think up what they want to say and be really active." (Respondent 9)

To sum up, the key idea regarding the presentation issues expressed by interviewees in the Australian case was that the videoconference presenters must be experts in their fields and, at the same time, be excellent communicators because they need to share their knowledge to a number of groups of multilingual, multicultural people with varying degree of knowledge on the matter. Informants also reported that the videoconferencing setting presents challenges even to people with excellent communication skills who are highly successful speakers in a face-to-face environment. Limitations incumbent on videoconferencing technology such as restricted mobility may cause frustration and affect performance. Interviewees felt that the presenters should strive for clear pronunciation, use comprehensible language and maintain an adequate pace of speech to facilitate more effective interpretation. At the same time, they expected the presenters to facilitate participation and encourage dialogue rather than focus on pushing the material to the audience. 


\section{2. The Russian Case}

The fieldwork for the Russian case was carried out in August, 2007 in Moscow. The data gathered included interviews with ten people aged between 27 and 55: university lecturers and management, government officials and staff of an international development institution (World Bank) who participated in videoconferences as facilitators, presenters, translator and regular participants. The respondents in the Russian case comprised nine Russians and one Armenian; three interviewees were women. All interviews, except one, were conducted in a face-to-face setting; the interview with a translator was carried out over the phone.

Unfortunately, during the Moscow fieldwork, no videoconferences were held at any of the GDLN affiliates thus I had no chance to observe live events. However, the Russian counterparts made available a few recordings of past videoconferences which were observed and notes used as additional data. Further data includes documents about two Moscow-based GDLN affiliates: the Modern University for the Humanities and the Moscow DLC co-located with the World Bank office.

\section{Cultural differences of participants}

Interviewees in the Russian case discussed cross-cultural communication from two different perspectives: interaction with people from the former Soviet states (domestic diversity) and communication with 'the rest of the world' (global diversity). Depending on their age, work and life experiences, the respondents had varying degrees of exposure to either type of communication. For example, a number of interviewees were university lecturers with over 20 years of lecturing experience in tertiary education. In the beginning of their career they taught students from 15 Soviet republics as well as students from socialists countries and developing nations in Africa that used to study in big numbers in 
Soviet Union. Now their students primarily comprise people from CIS 2 countries.

The majority of respondents in Russia felt that the different nationalities of participants had no influence on the effectiveness of cross-cultural communication in which they got involved during videoconferences.

"Such system of interaction, friendliness and willingness to share does not distinguish nationalities... Communication systems really unite people... these are real things, unforced, natural, that's what important." (Respondent 11)

"All people in the world have universal foundations. Major religions are based on the same principles... Of course, each nation led its own way from these founding principles. But the base stays and that base allows people to understand each other well enough." (Respondent 13)

“During such events [videoconferences], people don't have enough time to connect from a cultural difference point of view... Especially if the topic has been known in advance ... people meet already geared up with some templates or views." (Respondent 34)

Nevertheless, one of the respondents noted that national cultural differences were noticeable during videoconferences. His example applies to videoconferences that involve participants from CIS countries:

"It's very interesting when for example, we organise events in Russia that involve our branches in the Northern Caucasus. They have a very unique culture which is different from the rest of Russia. In general, they are quite explicit on something they don't like ... have no hesitation in expressing his/her own idea even if the presenter is a very prominent person." (Respondent 14)

\footnotetext{
2 The Commonwealth of Independent States (CIS) is the organization of former Soviet republics that was set up in December 1991; participating countries include Armenia, Azerbaijan, Belarus, Kazakhstan, Kyrgyzstan, Moldova, Russia, Tajikistan, Turkmenistan, Ukraine and Uzbekistan (Meyerson, Weick, \& Kramer, 1996)
} 
Another respondent - a translator - also agreed that cultural differences can be evident when people illustrate their talk by examples from their own cultures which might not have the same meaning or implication for people from other backgrounds:

"[Cultural differences] can only be noticed when someone brings up examples or realities from their own countries. For example, just mention "nine-eleven" ... [this phrase] may have a huge significance but [people from other cultures] might have no idea of what he is talking about... because that's the reality of another country." (Respondent 14)

Some interviewees believed that interaction during videoconferences was an unbiased dialogue among peers who were interested in exchanging professional experience and knowledge:

"[Communication during GDLN events] is ... more a peer-to-peer exchange." (Respondent 10)

"I need to note that there was no domination, no superiority of any one country over another but a very equal discussion was going on." (Respondent 13)

One of the respondents, whose professional capacity involved regular interaction with his GDLN colleagues, shared a story that illustrates how relationships are formed after people first meet through videoconferences.

"At first you communicate with them through videoconferences then once in half-a-year or once a year we meet face-to-face and then you approach them with trust, consult on any matters and they also treat you exactly in the same way. So in this regard, there are no barriers if only you are not making them." (Respondent 16)

Another interviewee also proposed that people who once met via a videoconference - just another electronic mean of communication along with voice calls over internet or email - would feel like acquaintances when they meet face-to-face: 
"Naturally, they will consider themselves to be acquainted because they already have something in common even if it is a participation in the same event and it doesn't matter if they have never seen each other before [in face-to-face setting]." (Respondent 34)

The above examples suggest that videoconferences may set an excellent foundation for later face-to-face or online interactions that could potentially lead to fruitful work and personal rapport between the participants.

Videoconference facilitators and participants agreed that communication between like-minded professionals involved minimal concerns regarding cultural differences because most videoconferences were organised on specific topics and involved practitioners and experts that worked in the same field, although in different countries.

“The main goal for us as organisers of videoconferences is to connect people that are interesting to each other from a professional point of view: their experiences may vary and be equally relevant and interesting to the other parties." (Respondent 10)

"In searching for participants we are not concerned about their cultures or what cultures they belong to but we are only interested in them as experts and look at the level of their expertise. Professional qualities of a person are of more interest to us than the cultural specifics." (Respondent 14)

“Cultural differences in terms of languages, habits, traditions... I didn't feel any, on my topic for instance. In general, governments perform similar functions ... I didn't feel any big cultural differences." (Respondent 12)

"We communicate with highly qualified professionals - government officials and experts - therefore, in my opinion, national cultural differences do not affect the understanding of what's going on." (Respondent 18)

The same argument was applicable to videoconferences that involved participants from Russia and other international audiences that connected from DLCs in other foreign countries as well as in CIS countries: 
"Firstly, as a rule, all the participants speak Russian, perfectly. Secondly, they all know the topic. Therefore, who cares how they look like? Frankly speaking, I don't pay any attention. I'm more interested in a [participant's level of] understanding of the topic, of the process rather than whom I'm talking with, what culture he represents, of what nationality he is, etc." (Respondent 15)

The respondents referred to the demographics of Russia, past and current, in justification of them being not so focused on the national cultural differences that exist in their society.

“In Russia ... so many different cultures coexist; practically all world cultures are represented in Russia. Though, for Russians it is considerably easy because they have a practice of communicating with people of other cultures." (Respondent 13)

"We constantly communicate anyway; we live in a multi-national country, that's all. It's just natural." (Respondent 15)

Another respondent shared his observation of how participants from different cultures perceive humour differently. This happened during a videoconference on e-government and e-services that involved World Bank experts and government officials from Russia and other CIS counties:

“A WB expert presented an example of an Arab country where a husband can notify his wife about the divorce by a text message from his cell phone. Well, it's an interesting and a funny example of IT applications in real life. However, the government officials in one of the participating countries took this example as an offence. They thought that by bringing up such a hilarious example, the WB expert didn't take the audience seriously... [One of them] whispered "they must be taking us for barbarians" implying "... they should've mentioned more serious examples of e-government". So this is an example of cultural differences if you want." (Respondent 10)

According to Bell (2007), humour is culturally complex and sophisticated thus posing a challenge for non-native English speakers. Sometimes attempts at humour may fail and cause offence in intercultural interaction. As rightly noted 
by Michailova (2000, p. 99), Russians and Westerners differ not merely in terms of national culture, but also in the economic, political, ideological, religious and social systems from which they come.

The respondents imply that people in Russia and CIS countries share the same mindset which is different from a 'western' mentality. However, a difference of mentalities is less obvious when cross-cultural communication involves professionals and is focused on a concrete subject of mutual interest.

"Countries in CIS had the same higher education system and people were raised in the system of the same mindset. However, the majority of communication [during videoconferences] is held in the context of western mentality... I would say, probably Russia belongs more to the Eastern mentality. Since we deal with professional communication..., it influences and to some extent it smoothens up the difference of mentalities." (Respondent 10)

The above respondent's belief that Russians have an Eastern mentality was rooted in the remote past history of Russia. Shkaratan's (2007, p. 100) quote explains it as follows: "There is no getting away from Russian mentality predominantly represented by the Russian version of Oriental Christianity. Nor can we ignore a national culture bearing traces of Oriental cultures, an influence that many historians have dubbed "the interaction of forest and steppe," the impact of the Mongolian yoke and subsequent influences by nomadic peoples who contacted the Russian settled civilization." Numerous studies attempted to uncover the nature, specific features and characteristics of the Russian mentality (Andreev, 2008; Elenkov, 1998; Muzykantsky, 2005). However, the scope of this study does not permit in depth discussion on the topic of differences between Russian and Western mentalities although it could have implications for cross-cultural video-mediated communication.

Another respondent thinks that intercultural communication that involves people of different nationalities that once used to be parts of Soviet Union would not be problematic because they share common values and have a similar mindset: 
"When we are talking about intercultural communication with the students who were born in the USSR and whose parents inherited a lot from those [Soviet] times, then this problem is not a hot issue ..." (Respondent 17)

Several informants made it clear that a different level of economic development among participating countries and consequently, a different level of knowledge of participants on specific topics has more influence on the effectiveness of cross-cultural communication than national cultural differences alone. Such a viewpoint is applicable to communication that takes place between CIS countries as well as multicultural interaction on a broader international level.

"The primary influence is not due to the cultural differences [of participants] but due to different levels of development and knowledge on a topic of a dialogue, communication or discussion in a particular country." (Respondent 10)

"All [participants] of course understand that some external stimulus is needed for the situation [in the region] to further develop because there, from what they [people, media] say, the situation is in stagnation, no development." (Respondent 14)

According to one of the respondents who frequently organised and facilitated GDLN events, the difference in the economic development of countries was an important consideration when they identify sites to be invited to join a particular videoconference.

“There shouldn't be a mismatch, both real or perceived. Perceived mismatch is when the client for whom this event is being organised doesn't perceive the other country/countries as peers and [that country's] experience is relevant to [their own] country. Often we need to consider that." (Respondent 10)

This respondent mentioned several examples of 'mismatch' when their clients were unhappy about the organiser's selection of countries.

"Normally such problems occur between CIS countries and African countries because differences can be too large in terms of levels of 
knowledge on a topic for an effective productive discussion..." (Respondent 10)

According to him, a group of government officials from one of the CIS countries felt insulted when the World Bank organisers invited them to join a videoconference on software outsourcing with a number of countries including a particular country from the Africa region.

"They thought that in Africa it [off-shore programming] is only at the embryonic stage whereas in their country the IT industry is considerably well developed. They've got a solid infrastructure. They assumed that the fact of inviting them in one videoconference together with that African country will send a message to the international community and their overseas partners that the WB considered the two countries to be on the same level. And because of that, many people refused to participate." (Respondent 10)

The above quote continues the theme about the uneven level of economic development among participating countries discussed earlier and corresponds with Hofstede's (2001) culture gap between developed and developing world and its illustration in a videoconferencing environment.

The following story reported by one of the informants suggests that power distance between superiors and subordinates (Hofstede, 2001) can be less apparent in videoconferences compared to face-to-face meetings:

“There was a bit of a tense atmosphere during videoconferences because participants here in Moscow were bosses whereas at the other sites were their subordinates... Maybe because it was a videoconference, people in the regions didn't feel pressured by Moscow. It was more open than a conversation that would've taken place if these officials were called to come to the ministry office in Moscow and talk face-to-face. All sorts of questions were asked, even some questions that would've not been asked if the communication was face-to-face." (Respondent 13)

Overall, responses of interviewees in the Russian case indicate that different nationalities of participants have no effect on the effectiveness of cross-cultural 
video-mediated communication although this may be evident through the ways people express themselves and how they interpret expressions and situations. Communication during videoconferences is a two-way peer-to-peer exchange of ideas and experiences rather than a one-way transfer of knowledge. Constructive dialogue among professionals and colleagues on specific topics left no room for concern regarding cultural differences of participants. Interviewees noted that people from the former Soviet Union shared the same mentality that differs from a 'Western' mindset. However they reported that it had minimal impact on video-mediated communication. The level of participants' knowledge on the topics of discussion had more influence on the effectiveness of cross-cultural communication than national cultural differences. It was suggested that the difference in the economic development of countries should be an important consideration when organisers select countries that will be invited to join a videoconference. A story told by one of the interviewees implied that videoconferences can be a stepping stone for further development of relationships between the participants. Another example illustrated that the power distance between superiors and subordinates can be less apparent during videoconferences compared to a face-to-face setting.

\section{Language differences and translation issues}

Research participants discussed problems of language barriers predominantly in the context of cross-cultural communication involving Russia and the rest of the world because most GDLN events are held in English.

"As a rule, the problem is that not everyone in Russia has an adequate level of English ... therefore synchronous interpretation is essential." (Respondent 14)

"The problem of language barriers [exists] because the majority of officials in CIS countries ... well, just a few can speak [English]" (Respondent 10)

"People in the audience might understand English well but have different cultural roots; they might have religious differences, differences in principles and values and so on. Of course, such a situation significantly 
complicates the mutual understanding on issues that are being discussed during videoconferences." (Respondent 17)

The issue of language differences was also relevant to the interaction among people from CIS countries, even more so now compared to the early post-Soviet era:

“Our compatriots are now returning to Russia, many of them ${ }^{3}$... People in Tajikistan and Kyrgyzstan - especially the younger generation - have problems because they don't know the Russian language." (Respondent 11) Most respondents noted that the translators played a vital role in the effectiveness of cross-cultural communication by minimizing misunderstanding and balancing the cultural differences of participants:

“Translators usually smooth out a little bit, don't convey all the emotions that are expressed by people in words." (Respondent 14)

"If the interpreter is highly qualified, if he understands problems of crosscultural communication and if he is a professional in the field of discussion then of course that person will largely reduce the problems of cross-cultural communication because he'll smoothen up some elements, translate some words in a way that is more appropriate for the other culture." (Respondent 17)

An interviewee who was often hired to translate for GDLN videoconferences described the situation from her professional point of view, revealing that in reality, translation-mediated speeches were smoothed out just because of the technicalities of the process:

"In synchronous translation, only the general meaning is transmitted... Frankly speaking, only the subject, predicate etc: who? did what? when? [are translated]. All other parts of speech such as parenthesis and some emotional utterances are omitted." (Respondent 34)

\footnotetext{
${ }^{3}$ On June 22, 2006, Russian President Vladimir Putin issued a decree initiating an ambitious six-year program designed to facilitate the voluntary repatriation of some of the estimated 30 million Russians living abroad, both in the CIS and further afield (Peuch, 2007)
} 
Participants who require translation can't hear the actual speech having no other choice but to listen to the interpreter's version. Research conducted by Collados (1998) discovered that the interpreter's monotonous intonation has a negative impact on listener's perception of the quality of interpretation as well as their impression of the interpreter's professionalism and reliability (Pöchhacker, 1999).

"We often have synchronous translation which does not allow the presenter to express [their emotions] in full because we don't hear his voice, we hear the translation in Russian." (Respondent 14)

"When someone talks, even makes a presentation, it can be monotonous. Especially when we're using synchronous translation, it can be even more monotonous. Emotions, not all the emotions of the presenter are conveyed when translation is involved." (Respondent 14)

On the other hand, translation-mediated communication is succinct and formal. A simultaneously interpreted version can be more concise than the original speech because of the source language.

"Synchronous interpretation compresses and shortens the presentation to some extent, to the summary." (Respondent 13)

The quality of interpretation services was evaluated by users in terms of what they actually received in relation to what they expected (Kurz, 2001). However it was not clear what factors influenced the perception of that quality, since universal tests to objectively determine quality perception were yet to be designed and users do not know the departure language (Kahane, 2000). Most respondents admitted that the quality of simultaneous interpretation is inconsistent because finding excellent interpreters can be a difficult task. Moreover, a good interpreter was someone who has excellent language skills and adequate knowledge on the topic of discussion. While the ideal situation requires interpreters to specialize in a given field, conference organizers often perceive the interpreter to be a "Jack of all trades" (Al-Qinai, 2004, p. 64). Sometimes, organizers need to step in and assist the interpreters in finding the appropriate term or providing clarification on the matter under discussion. 
"Finding a quality interpreter is a big problem. Because it is not always possible to get the translator [who is specialized] on the topic, not always he/she conveys the ideas accurately ... Sometimes my WB colleagues ask me to translate although I'm not a professional interpreter, but I know more on the topic... I can't do synchronous interpretation. However, I could probably convey the message more accurately." (Respondent 10)

"We always try to invite highly qualified interpreters whom we know, whom other people have recommended to us, who have worked with us a lot, well-proven." (Respondent 13)

Mack and Cattaruzza (1999) established that the 'ideal' performance should be terminologically correct and informed, accurate and easy to follow. However, the following responses illustrate that the quality of interpretation during GDLN videoconferences did not always reach the desired level:

"The presenters assume that the translators are conveying their ideas accurately but [if the translation was poor] the participants who receive information would assume that the presenter had an inadequate understanding on the topic on which they claimed to be experts. That's only because of the translators; the quality of translation." (Respondent 14)

"Sometimes I feel that the question wasn't translated accurately when the presenter's answer is not matching the question that was asked. Therefore the translators are essential and they should be highly qualified, they should have an understanding on the topic of discussion." (Respondent 12)

“People who don't know English think that they're getting an accurate translation. But when you see that the translation is poor, when the person is struggling ... very few people, actually just 1-2 individuals are proven, whom we can rely on..., the terminology that we use is very specific." (Respondent 16)

In addition, the quotes above entail several important issues such as discrepancies between the interpreted version and the original that are hard for listeners to judge, as they do not know both languages (Kahane, 2000); and that erroneous rendition might lead to an unfair assessment of speakers' 
competence by some participants. Though research suggests that it is difficult to establish what the conference participants expect from interpreters and how satisfied they are with the service they receive, different listeners in the same situation may have different expectations (Kurz, 2001).

Interviewees agreed that quality interpretation during videoconferences required adequate preparation in teaming up organizers and interpreters. They suggested that the organizers and the interpreters alike should invest some effort prior to the event so that the interpretation during the actual session was flawless.

"The translator must be given, in advance, the glossary on the topic, main terms and definitions. Synchronous interpreters are professionals. Nevertheless, they should be given prior orientation on the topic [so that they can] process the information and clarify issues before the session if they have any questions." (Respondent 15)

“Even an excellent professional can't pick it right away, he needs to prepare to get it [right]." (Respondent 16)

“Interpreters also must be well prepared not only in terms of language skills but also in terms of cross-cultural communication. It's very important because their incorrect translation, absolutely unintentional, might create severe communication problems." (Respondent 17)

The organizers understood that the provision of quality interpretation services came at a price but agreed that simultaneous interpretation was hard work and should be paid accordingly especially because a professional in this field is a scarce resource:

"It is very expensive: we pay the interpreters up to twelve hundred dollars for a two-hour session ... interpreters always work in pairs... But they do work-off that money, absolutely. They really work hard." (Respondent 13)

"Synchronous interpretation is the most expensive translation ..., we have to involve professionals; there are very few professionals who could 
perform a quality synchronous interpretation. Well, since not many people

[can do simultaneous interpretation], they charge a lot." (Respondent 16)

Technical facilitation of simultaneous interpretation during videoconferences can be a challenging task: poor sound quality, malfunction of equipment in the translator's booth or the videoconferencing system might distract the participants and seriously jeopardize the successful accomplishment of videoconference objectives.

"Synchronous interpretation is a very complex process technically: any slipup by the sound technician gives a very unpleasant effect when you hear both the presenter and the translator at the same time. That is very disturbing." (Respondent 13)

"While simultaneously interpreting a video session, the interpreter may not catch and simply miss the idea because of the noises and distortions in video or audio channel." (Respondent 10)

In sum, the responses of Russian case interviewees imply that the majority of participants joining GDLN events from Russia have limited English language skills and thus fully depend on simultaneous translation services. The translators play a vital role in minimising misunderstanding and bridging the cultural differences of participants, although the simultaneously interpreted version can be more laconic and less emotional compared to the original speech. Several informants noted that highly professional and reliable simultaneous interpreters were scarce and their services were expensive. Interviewees acknowledged that most videoconference participants cannot assess the quality of interpretation because they don't know the language of the original speech and consequently, they can make wrong judgements about the speakers based on the version they hear. The participants expected the translators to have adequate knowledge on the topic of discussion but that was not always the case. At times the organisers needed to step in when interpreters struggle with specific terms or complex ideas. The informants believed that prior preparation and cooperation between translators and organisers will 
enable flawless translation during the actual event. However simultaneous translation can be hindered by technical problems such as poor sound quality.

\section{Facilitation and moderation issues}

In contrast with DLC in Australia, the two Russian centres were normally on the content-receiving rather than content-generating side. Nevertheless, facilitating videoconferences in Russia seemed to be a complex and rather political mission. The videoconference facilitator was responsible for the overall outcome of the event because he/she played an important role in choosing the participants, presenters and interpreters as well as managing a number of other equally significant tasks such as instructing the presenters, interpreters and participants, making sure that the organisation of the event fitted to the allocated budget and arranging for gathering participants' feedback after the session.

"The role of the local facilitator is actually to create a certain balance between the participants, adequately instruct the presenters so that they talk about things that are relevant to the local audience. You always have to arrange things in a way that people accept each other as peers and equal discussants. So you have to manage these issues on a case by case basis." (Respondent 10)

"Facilitator's activity is very comprehensive. It consists of making contracts, performing financial calculations, inviting guests [speakers], selecting participants, developing forms, questionnaires, etc." (Respondent 13)

"The facilitator needs to select presenters of a certain standard who can tell something that's interesting enough to hold the audience... He/she should gather people who have some knowledge on the topic and are ready to take part in the discussions. [The facilitator] should bear in mind that the communication must be a discussion rather than a one-way communication." (Respondent 18)

Respondents agree that the moderator played a vital role in the effectiveness of videoconferences. They can make or break a successful event. By effectively 
chairing the discussion and keeping everybody motivated and engaged, the moderator can significantly improve participants' satisfaction.

“The moderator can significantly improve the effectiveness... by linking up comments of various people, making summaries and drawing conclusions that can lead the discussion in more productive way." (Respondent 10)

"Although in general the people who come here do so because the topic is interesting to them, right? But if - in addition to that - the moderator is able to intrigue you, to capture your interest, then the effect is much better." (Respondent 14)

Moderators ensured that the session followed a predefined running order. They chose questions to be aired, made floor control decisions during Q\&A sessions, stepped into the discussion with competence, improvised and filled in the gaps caused by technical problems, handled administrative issues, etc.

“The Moderator's role is to keep everyone interested, maintaining the right combination and right balance of questions from participants. In case of various technical difficulties he/she should be able to entertain the public..., make sure that the speaker is not going over the allocated time." (Respondent 10)

"The Moderator... makes sure that the time schedule is followed; ... interrupt a speaker if he goes beyond given time. If this conference is being streamed live on the internet, the Moderator looks at the questions that come up through the internet and reads them out to the presenters. Then the moderator also makes admin arrangements: tells people where to get the power points, etc." (Respondent 12)

"... see whom to give a floor, who wants to say something, who may have an interesting question." (Respondent 13)

"He should unite the audience, fill the gap with himself... should be a very knowledgeable [person] in order to tell something on the topic that's being discussed." (Respondent 14) 
Respondents, who often act as moderator and facilitator at the same time, illustrated how overwhelming it can be to juggle many tasks simultaneously:

"Sometimes I don't have enough time, I have no possibility to attentively listen to the discussion because all my efforts are spent on monitoring the time that is left to the speaker, look around and figure out the participants who are ready to ask questions, observe the reaction of the listeners, in parallel solve some technical problems, often we simultaneously receive questions via internet using chat system..." (Respondent 9)

"I can feel that the audience is getting bored and then I try to revive the situation somehow, interrupt the presenter by asking him a question or switch to another site so that they can ask a question." (Respondent 13)

"During a videoconference the Moderator wears out much more energy than any of the presenters. It's a very difficult mission, hard work. ...demands lots of effort, consumes plenty of energy, psychological energy." (Respondent 17)

One of the respondents who regularly facilitated sessions but had no experience as a moderator made an observation that pointed out a significant difference between the two functions - while moderators acted in the limelight, the facilitators' performance was often left unnoticed and taken for granted.

"When we're praised for a [successfully organised] event, the moderators are the ones who are getting the most acclaims [laughs]." (Respondent 14)

While facilitators mainly work off the screen, the moderators have no mercy if something goes wrong because they act in live, real-time communication.

"[When] he has a sluggish kind of look, not a very enthusiastic kind, maybe not in mood .... But that affects [the effectiveness of videoconference]." (Respondent 14)

Regular participants acknowledged that moderating a video-session was a very complex task.

"Of course, it's not an easy role, requires training. ... It's a very complex activity." (Respondent 12) 
“The moderator must be a super-professional ... intuitive... ready to answer any question." (Respondent 15)

"It must be a very talented person...must be very experienced, have certain skills, keep within the timeframe but let people tell what they want, make compromises, maintain balanced communication and finish the videoconference on time but be able to tell everything what was intended." (Respondent 16)

To sum up, the facilitators of videoconferences in the Russian case juggle many important tasks to make the event possible: not only during the actual videoconference but also well in advance as well as after the session. They primarily work off the screen whereas the moderators work in the lime light and often get praised if the event was a success. Many respondents agree that the moderator plays a key role in the effectiveness of communication that takes place during videoconferences and that his task is very complex. The interviewees believed that people with outstanding interpersonal skills and a great sense of tact and time can enable effective communication by competently moderating the sessions..

\section{Presentation issues}

Respondents in the Russian case agreed that the role of presenter was extremely important for the effectiveness of videoconferences as it was for any conferences in a face-to-face setting. According to Respondent 10, the speakers must be talking at a level appropriate to the level of knowledge in the target audience. However, it was not always easy for the speakers to know that in advance. They may not get enough information or the actual level of participants' knowledge could be lower than expected.

"It can be that the videoconference was not so interesting ... when the speaker doesn't very well present the topic as he could have [and/or] when he is not aware of the knowledge level of the audience." (Respondent 10) Respondent 14 recalled an example from a videoconference in which one of the connected sites was a city in Northern Caucasus. A World Bank expert made a presentation introducing some recommendations for development of small- 
scale businesses in the region. However, during the Q\&A session, one of the participants openly critiqued the presenter for not having enough knowledge about the region:

"Someone who took the floor commented that the presenter who was talking about the Northern Caucasus does not have enough knowledge about the region to be able to advise [what to do]." (Respondent 14)

The quote above suggests that in some cases, the level of knowledge of some presenters does not match the expectations of the participants.

Good communication and presentation skills of the speakers will have a great impact on the participants' perception of the effectiveness of videoconferences. However, being a good presenter in a face-to-face setting does not mean that the speaker will perform equally well in a videoconferencing environment.

"Of course, the presentation skills of the speaker have a huge impact. I mean the presentation skills not only in abstract terms, in terms of working with audiences in general but particularly working during the videoconferences. Because here we have some specifics, let's say pedagogical specifics that are not always considered by the speakers." (Respondent 10)

In contrast, Respondent 14 thought that the speakers' presentations or communication skills were less important in videoconferencing than in a faceto-face setting. He thought that the style of communication was less formal during videoconferences than during face-to-face conferences and the content of presentation mattered the most.

"If the topic is interesting then anyone, even someone who stutters or is not a very good speaker, can attract participants' attention.... as for personal communication skills, I think here [in a videoconference setting] they come down to zero because he is not presenting on the stage before people. Here what he's talking about is much more important than how he's talking (emphasis added)." (Respondent 14) 
Many other interviewees agreed that the content of the presentation mattered the most. Participants wanted to hear new information on the topic from a credible and experienced person who knew the topic well.

"We consider whether the person is well recognised in the field, has professional experience in this field, etc. Usually these people have vast experience in working with audiences." (Respondent 10)

“The presenter in a videoconference ... determines the whole thing. Interest to the videoconference and how informative it is depends on the selection of quality presenters." (Respondent 12)

"The presentation has to be very informative and interesting, filled [with facts]." (Respondent 15)

Respondent 17 was the only one who mentioned that the presenters should have cross-cultural competence in order to successfully communicate ideas to multicultural audiences.

"It's important to make sure that the presenters are well prepared. They should be professionals not only in the field of the discussion but also be aware of problems of cross-cultural communication." (Respondent 17)

A few interviewees reported that the effective running of a videoconference depended considerably on the presenters' ability to fit his talk into the time given. Respondents 10 and 12 - both experienced videoconference moderators provided examples of dealing with overly talkative presenters in a videoconferencing environment. One thought that a video channel made it harder to interrupt speakers whereas his colleague found videoconference mode more advantageous in this regard than in a face-to-face setting:

"Sometimes a person is so deeply engaged in the topic it's impossible to stop him. During a real [face-to-face] seminar it is possible to give a hint to the presenter... whereas during a videoconference it is more difficult because usually it is a voice-activated system so when someone starts talking sometimes there is no other way to stop him but just cut off that site." (Respondent 10) 
"In fact, it's another advantage of a videoconference connection because if someone is presenting on the stage, from the pedestal, you cannot drag him by his legs and take him away [laughs]. But during a videoconference, as a moderator, I can ... interrupt the presenter by asking him a question or switching to another site so that they can ask a question." (Respondent 13)

Interviewees, who participated in videoconferences as moderators as well as those who took part as regular participants, mentioned situations when the moderator needed to interrupt the presenter because either he exceeded his time limit or the audience was bored.

“Normally it doesn't look nice when someone from another site is trying to stop or interrupt the speaker - it looks like mismanagement, miscommunication or there is no understanding between the speaker and the videoconference organisers." (Respondent 10)

"If people are bored, you need to interrupt and do something. ... without offending a person, delicately but persistently at the same time, in order to keep the audience interested." (Respondent 13)

A couple of interviewees discussed the issue of presentation during videoconferences in comparison with a face-to-face situation. For instance, they mentioned that the presenter's task became much harder in a videoconference setting because he or she was unable to establish eye contact with the audience. As a result, some presenters focussed on the presentation slides as a way of coping with this limitation.

"The problem here is that ... in a live audience you can combine slide presentations on the screen and maintain eye contact ...But during videoconferences as a rule, you get either the slides or the face of the presenter on the screen. ... The problem is that then the presenter focuses too much on his slides." (Respondent 10)

"The specific of a videoconference is that the presenter cannot feel the live audience. They can only bring in some feelings during the Q\&A sessions when answering questions or explaining something." (Respondent 12) 
According to a quote by Respondent 12 above, videoconference presenters cannot be fully aware of atmosphere during the actual presentation but can get some indication of participants' reaction to his talk when they start asking questions or provide comments.

The technical set-up of the room and the positioning of the camera angle has a significant influence on the effectiveness of presentation, thinks Respondent 10.

"The technician who sets-up a videoconferencing room should make sure that the camera is located just above the monitor because he [presenter] instinctively looks into the monitor [because] he sees faces of people on the screen and he looks at them. If the camera is placed on the side, the other participating sites will see the presenter looking somewhere else. So it plays a role, too." (Respondent 10)

Perhaps, he was talking about the arrangement that was applicable to the setting of a DLC where he normally took part in videoconferences. Nevertheless, the message he was conveying was that the camera should be placed in a position and at a level that best captures the speaker's front view while he or she is looking at the screen displaying images of participating sites. This would at least give the participants an impression that they are being looked at by the presenter:

"... so that the other sites would perceive the dialogues as live, more close to the real [face-to-face] situation." (Respondent 10)

Technical troubles can ruin the impression about the effectiveness of a videoconference-mediated event.

"Sometimes you have to blush, the effectiveness of the event is strongly diminished and energy wasted because so much effort was made to invite the experts, collect participants in the audience, whereas due to some technical problem all these efforts could come to nothing." (Respondent 10)

Sometimes presenters felt uneasy in front of a camera, reported Respondent 13. As a moderator, he saw quite often how hard it was for some speakers. So he came to help when, in his opinion, it was needed: 
"Sometimes when we invite experts who get to see the camera for the first time, I can see how difficult it is for them. Sometimes you have to interrupt a person because he looks at you and his eyes beg you to stop him talking. He wants to have some water but he is uncomfortable doing that in front of a camera." (Respondent 13)

"Naturally maybe only during the first few minutes, when the format of the event is introduced, some tension can be felt." (Respondent 10)

However, technology apprehension disappeared fairly soon - noted both respondents - and it was more likely during the presentation than the Q\&A sessions:

"It's almost gone during the first session, towards the end. When the Q\&A session starts, when dialogue starts, it goes right away. (Respondent 13)

Normally, this [tension] disappears quickly." (Respondent 10)

To summarise, respondents in the Russia case considered that presentation played a significant role for the effectiveness of cross-cultural videoconferences. A few interviewees discussed presentation issues in comparison with a face-toface setting. A number of informants suggested that the videoconferences achieve the best outcome if the audiences' level of knowledge on the subject matter matched the presenter's expectations. It was noted also that the presenters should be very competent in order to meet the audience's expectations. A number of respondents thought the content of the presentation was crucial for the success of a videoconference as for any other conference. They agreed that the presenter - someone prominent in the field - should make his presentation as informative and interesting as possible in order to capture the attention of remotely located audiences. Given the strict time limitations during videoconferences, he or she needed to fit into the allocated time. Several conflicting ideas regarding a number of issues emerged from the interview data. For example, some interviewees thought that the presentations skills of the speakers mattered more in a videoconference setting than in a real life situation whereas others reported the opposite. One respondent believed that the videoconference setting made it harder to interrupt speakers who exceeded 
their time limit whereas another said that it was easier to do during a videoconference than during a face-to-face conference. A few interviewees noted that presenting during a videoconference can be challenging because the speaker was unable to make eye contact and feel the atmosphere at distant audiences. However, they can get some indication of participants' reaction when Q\&A sessions begin. The technical set up of the videoconferencing room, especially the positioning of the camera, could help in reducing this downside by creating an illusion that the speaker was looking at the participants when talking. Technology apprehension was another problem that bothered some presenters during videoconference, but they usually got used to the set-up fairly quickly, commented the interviewees. 


\section{3. The New Zealand Case}

The fieldwork for the New Zealand case was carried out in January-February 2008 in Wellington. The data gathered included interviews with six participants of videoconferences - Victoria University lecturers and management staff as well as government officials - men and women aged from 28 to late 50's. They participated in videoconferences with different roles. Some facilitated the events as well as made presentations whereas others attended videoconferences as regular participants. The group consisted of New Zealanders of European descent including those who emigrated from the UK and Austria. Their videoconferencing experience ranged from just one session to numerous crosscultural events - specific operational meetings and more general discussions that took place via videoconferencing technology. Operational meetings that took place through GDLN normally involved people of different national and organisational cultural backgrounds.

Additional resources in the New Zealand case included notes on the observation of three videoconferences: one session on anti-corruption strategies that was discussed by a number of respondents in both the New Zealand and Australian cases, and two other sessions that took place after the interviews had been completed.

\section{Cultural differences of participants}

Discussing the issue of cultural differences of participants, all interviewees in the New Zealand case noticed the differences of cultures at national level whereas some of them noted the differences at organisational level as well. In general, they agreed that the apparent differences of cultures did not present barriers for communication.

"It was quite evident, the differences ... in both the general culture and the workplace culture... and not that any was right or wrong, or better or worse... it was just ... people's body languages, the way they talk." (Respondent 22) 
However, the following respondent firmly believed that the communication was hindered by differences in the levels of knowledge and experience among the participants rather than by national or organisational cultural differences.

"The other difficulty ... is that the experience of the various nations was extremely different. So, you have Australia and New Zealand well versed in modern fisheries management and you had a number of other nations with traditional fisheries. ...So it was in some sense cultural but in some sense simply a knowledge and experience gap." (Respondent 19)

The above respondent explained that the topic of the series of videoconferences on fisheries management was too advanced and inapplicable for the countries with traditional fisheries like Fiji, Papua New Guinea and Timor Leste. Nevertheless, Respondent 19 was hopeful that the participants from Pacific nations had benefited from the discussions through hearing new information and learning how fisheries are managed in other countries with which they share the waters. He was confident that the video-discussion was a worthwhile initiative because people had an opportunity to talk about common problems and acquired new knowledge.

"I think they got some new knowledge whether they can use it or not, I'm not sure. What I think they probably did get is an appreciation that the problems they're facing are being faced by others. So that in itself is actually worth the forum." (Respondent 19)

The issue of the knowledge and experience gap was brought up by another interviewee who attended a videoconference on the subject of public-private partnership in infrastructure financing. In his perception, such a disparity affected the effectiveness of the discussions more than merely cultural the differences of participants.

"It wasn't ... cultural differences that affected the discussion. It was more of a level of knowledge about the topics that the people from some of the island cultures ...basically had very little experience with, and little knowledge with some of the infrastructure financing issues that were being discussed..." (Respondent 20) 
Respondents 19 and 20 described why they got an impression that the level of knowledge and experience among the participants was different. For example, one of them made assumptions based on the questions that were asked during the Q\&A sessions whereas the other one interpreted the quietness of some participants as an indication of lack of knowledge:

"And what we saw... a number of very basic fish questions which make perfect sense to the nation asking them, but didn't fit the ... more technical context of the presentation that was given." (Respondent 19)

"I think that is the main reason they were fairly quiet because they just didn't know much about it [infrastructure financing]." (Respondent 20)

On the other hand, the theme of mismatch in levels of participants' knowledge and experience demonstrated above may also imply that ineffective forums were being convened by the organisers.

The situation was different at the series of videoconferences on anti-corruption policies, according to one of the participants representing a government department. He got the impression that it was an interesting discussion among knowledgeable and experienced people and that bridged the cultural differences.

"It seemed they were either experienced professionals or academics, so they were all relatively familiar with the topics and in that sense I think it reduced the cultural boundaries." (Respondent 22)

This impression was shared by another interviewee, a university lecturer, who attended the same series of videoconferences. He also thought that all participants were on the same page and found it to be crucial for the effectiveness of communication.

"In that particular case it was pretty much a shared understanding of what the issues were and we all in fact had a sense that we were talking about the same thing, which is pretty important in my experience." (Respondent 23)

My observation notes confirm that during the above-mentioned series of videoconferences the participants held wide-ranging discussions on anti- 
corruption practices in various sectors of the economy, brought up examples from their countries and exchanged ideas on ways of combating the problem. However, the impressions of respondents 22 and 23 from the anti-corruption strategies sessions contradict the experiences of Respondent 19 who attended the series of videoconferences on fisheries management topic and the Respondent 20 who attended a videoconference on public-private partnership in infrastructure financing.

A set of rules has been pre-established for the series of videoconferences on anti-corruption policies according to one of the respondents because of the number of sites involved and the different cultures represented. He understood that according to these rules, the discussion would be held in English and led by a moderator in Canberra.

"I think in that sense we were all playing by the same rules, but they were essentially Australian rules, English rules. I'm sure that if it had been led by someone from Fiji or from PNG, or from Timor, it might have been conducted very differently... Which I don't think would have been an issue as long as someone knew how that was going to operate." (Respondent 22)

By saying that, the respondent suggested that all participants, no matter what culture they come from, adhered to the rules pre-determined by a dominant culture. The interpretation of this idea could be that by doing so, the organisers streamlined the running of videoconferences and reduced the cultural boundaries. On the other hand, it could also mean that the designers of this event pushed forward an agenda that did not take into account the cultural diversity of the participants.

Another participant, who attended one of the sessions on anti-corruption policies, (it is not clear whether it was the same session that was attended by Respondent 23) comments that the discussion was dominated by a few people, mainly of European descent, whereas the participants from Pacific nations kept rather quiet. He proposed several possible reasons for some participants hesitating to engage in the discussions including national cultural attributes, power distance and/or personality features. 
"It could have been for a variety of reasons. It might have been cultural shyness, it might have been respect for a teacher or an older, more authoritative figure. It might have been just difference in personalities." (Respondent 24)

At the same time, he guessed that it might have been the videoconferencing setting that made these participants uncomfortable whereas it did not much bother their more talkative partners who happened to be white Caucasians.

"I do suspect ... it was because the European ethnicity person felt more comfortable engaging in a debate in that sort of environment than did the other people who were from a range of ... Melanesian and Micronesian backgrounds. And I suspect, and I'm only guessing, that they felt more uncomfortable engaging in debate and dialogue in that sort of environment and probably that being a videoconference environment made it more of a factor." (Respondent 24)

The above informant recalled that "one participant in Australia National [University] always dominated the discussion and other people just listened" which must have been the Moderator who was operating from the Canberra site, according to observation notes.

Respondents 22, 23 and 24 attended one or more videoconferences from the series entitled "Anti-corruption strategies: practical implementation" that consisted of four sessions in total. Respondent 24 attended just one videoconference and felt that the discussion during that particular session was dominated by few people, mainly of European descent. At the same time, two other people - Respondents 22 and 23 who attended two or more sessions noted that the participants shared a common understanding and seemed to be knowledgeable and experienced people. However they provided no comments about discussions being dominated by a small number of people.

One of the respondents felt that the discussion would have been livelier and more productive if the participants had a chance to get to know each other better. According to him, at the beginning of the session the moderator asked 
the participants at each site to introduce themselves but it did not help people to open up and start talking.

"It was all very formal and stiff and I think a lot of people ... especially some of the others [from Pacific islands] were quite inhibited about speaking or sounding stupid ..." (Respondent 20)

According to one of the interviewees, New Zealand as a nation has developed a very good understanding about cultural differences that became a custom and normal practice in people's lives.

"I think New Zealand as a nation, we've come quite a long way in terms of our own [understanding]... of the issues that we had to acknowledge around ... cultural and racial difference, so I think ... we've got something to offer in relation to that understanding." (Respondent 21)

This respondent - a New Zealander - also thought that the cultural issues in New Zealand are handled differently than in Australia. She explained that it was a professional requirement for white New Zealanders to learn and understand cultural issues and that established a society's expectation.

"There is quite a different relationship in dialogue with indigenous people here than there is in Australia and that spins off into a whole range of other issues in terms of other cultures and issues of respect and equity." (Respondent 21)

The above statement by Respondent 21 cannot be confirmed by research data and thus may or may not be true.

Another idea expressed by one of the interviewees arose while he was reflecting on cross-cultural communication taking place through video channel in comparison with face-to-face interaction. He implied that when someone travels to another culture and communicates with local people in their own habitat, which is so different from his own, he/she may feel socially uncomfortable. But when a meeting takes place via videoconferencing, the participants feel more comfortable because physically they are located in their usual environment although mentally they co-exist with other people in some 
virtual environment. Free of social anxiety, the participants can focus on the matter of discussion and effectively contribute ideas, believes Respondent 22 .

"In videoconferencing everyone ... is in their own environment and yet they are communicating with people from different environments who were at the same time very comfortable at home but because of this link up...they are able to interact. So I think ... that they might really benefit from the video link because when people feel comfortable they are ... more inclined to really open up and give their opinions and discuss." (Respondent 22)

Elaborating further, the respondent suggested that videoconferencing could be an excellent medium for mediating conflicts and discussing tense or sensitive issues. This idea supports the comments expressed earlier by some respondents in the Australian case that sensitive issues such as corruption were successfully discussed during videoconferences.

According to another interviewee, no matter what medium was employed, the effectiveness of communication depended on people's ability to consider cultural differences when taking part in cross-cultural exchange. She was certain that the organisers and presenters should know the audience and their needs, be aware of the important things that should be taken into account and skilled in encouraging participation, in order to facilitate a successful communication.

"If people aren't used to taking cultural differences into account in relation to a communication style or the way a learning situation is approached then their usual practice will be brought forward into a video conferencing situation. You'll get exactly the same dynamic, only worse." (Respondent 21)

Reflecting on his extensive videoconferencing experience, one of the interviewees noted that working meetings regarding various development projects that linked multiple sites and involved World Bank staff and their counterparts in different countries tend to be very efficient. Participants focus on a particular operational issue and were guided by the same rules so the cultural backgrounds of people did not pose barriers. 
"Culture did not really enter into that in a sense of culture derived from ethnicity... and there were no obvious specific cultural barriers to communication in that case." (Respondent 24)

He commented that the World Bank's organisational culture dominated the video-mediated conversation that took place in English and usually contained lots of institutional slang and complex terms. He suggested that nevertheless, their counterparts working on World Bank-funded projects in various developing countries - staff of international consulting firms, local government officials and other stakeholders - all operated under the same guidelines and effectively communicated with each other.

“The World Bank has a very strong technocratic culture, most of, but not all of the staff, speak that language... [International financial institutions] do bring together such a wide variety of cultures and there may well be an underlying organisational culture, but generally everybody working in them and contractors with experience will all share the same basic set of concepts and constructs." (Respondent 24)

The context of the interview suggested that the term 'culture' used in the above remark refers not only to the national culture but also to organisational culture. That exists especially at huge and complex international organizations such as the World Bank (Weaver \& Leiteritz, 2005).

The issue of power relations as articulated by Hofstede (2001) was raised by several informants. One of them talked about communication between big countries and smaller countries and the tendency of powerful nations dictating to other countries and small island-states in the region. He also pointed out the complexity of language that was often used in such communication.

"Because communication is coming ... from a large country, from people using really technical language... And it has the feeling that a larger country is telling us: "Here's what we should do." (Respondent 19)

While saying that, he pointed out that New Zealand managed its fisheries sector quite well and was far ahead of Australia in that respect. He commented 
on the specific relationship between Australia and New Zealand which he describes as follows:

“There's a friendly rivalry, certainly, there's also a sense in which it's a big country and we, in New Zealand at least, don't like being told what to do." (Respondent 19)

Another respondent recalled his past videoconferencing experience when he was involved in the series of operational meetings linking two project teams one in Auckland and the other in Wellington. He explained that the team in Auckland included not only staff from the same government department but also people from other stakeholders. Although the communication held during these meetings had a business context and was mono-cultural, the experience shared by this respondent might as well be applicable to cross-cultural communication in a development context.

"When the link was cut then we would talk to each other and ...often we would say things we weren't prepared to say in a bigger group... because there was a difference between the two groups." (Respondent 20)

A respondent, who attended the videoconferences on anti-corruption policies, implied power relations when he was explaining possible reasons for some participants having to modify their responses or hesitating to engage in discussions entirely. He pointed out the issue of trust in the group, especially when sensitive issues were being discussed and superiors were present. Perhaps, the following quote implied power relations in general, not only in relation to video-mediated and/or cross-cultural communication.

“Because you sometimes sense, “well, I don't want to say too much because I'm not really comfortable with the people I'm in the room with". They may not feel they can trust them entirely. That's a big issue in talking about corruption in other countries, for obvious reasons. And also, you ... often get a distinction between what people feel obliged to say, not [what] they actually think." (Respondent 23)

Another interviewee brought up an example from his videoconferencing experience that supports the ideas expressed by the quote above. He recalled 
that during an operational videoconference involving some senior officials one of his normally very vocal and opinionated colleagues, a Thai national, was unusually quiet. He explains:

"Thais are certainly not always kind of reticent, quiet people. They can be upfront about things and she was one and she had very strong views about the project. But sitting in the company of her regional director in Bangkok, it was very hard to get her to say anything [laughs]." (Respondent 24)

The above respondent's perception of Thai people contrasts with the value of "Kreng jai" (to be considerate), according to which Thais in general feel reluctant to impose, or make other people feel uncomfortable, and they always take other people's feeling into account (Jonjoubsong, 2008). However, the above example complies with high power distance in Thai culture where subordinates usually accord respect and feel obligations to their superiors (Burn \& Thongprasert, 2005).

In summary, the interviewees in the New Zealand case looked at the issue of cultural differences in videoconferencing setting from a number of perspectives. They talked about differences in terms of national and organisational cultures while reflecting on their participation in various types of cross-cultural video-mediated communication: project-specific operational meetings and more generic discussions on broad development topics. Most respondents agreed that the knowledge and experience gap among participants presents more barriers to effective communication than just differences in the national and/or organisational cultures they belong to. The gap was more noticeable during the videoconferences on complex topics such as the economics of fisheries management and public-private partnership in infrastructure financing. On the contrary, respondents who took part in videoconferences on a broader topic of anti-corruption strategies felt that all the participants were knowledgeable and experienced professionals and equally contributed to the forum. The issue of adequate introduction and ice-breaking for more productive discussion was brought up. According to some interviewees, one race tended to set the rules and dominate generic discussions whereas one strong organisational culture can be a common denominator for 
specific operational meetings. The videoconferencing setting might inhibit participation, according to some informants. The relationship between Australia and New Zealand and their approach to cultural issues was discussed. Issues of power distance were illustrated by specific examples.

\section{Language differences and Translation issues}

Some of the New Zealand respondents reflect on their experience of attending various sessions under the Pacific Leaders Virtual Forum, a program that was developed by the Australian National University and delivered via GDLN. The program documents and observation notes suggested that the videoconferences should be held in English and only one of the participating sites - a Distance Learning Centre in Dili, Timor Leste - was using simultaneous interpretation.

The majority of respondents agreed that the cultural differences of participants naturally presented language barriers and assumed that it may influence the effectiveness of communication to some extent.

“They were all English speakers although some of them struggled a little bit with the language... and there was a little bit of a barrier. (Respondent 20)

You couldn't tell because a lot of them didn't speak [laughs]. But that could well have been a factor." (Respondent 24)

While assuming that language differences could potentially impede communication, the respondents admitted that they had no other choice but to speak English because it was the most commonly spoken language among the countries participating in videoconferences. Probably the fact that New Zealand is largely a monolingual nation, with 80.5 percent of people speaking only English (Profile of New Zealander Responses, Ethnicity Question: 2006 Census, 2006) played a role.

“Obviously, the language barrier would've been a bit of an issue. English as a second language is obviously more prevalent in other countries than for example Timorese [language]. I guess it's just finding the lowest common denominator." (Respondent 22)

"Of course, our only mode of communication is English." (Respondent 24) 
On the other hand, there were respondents who felt that although evident, the language differences caused no obstacles in communication.

"[Difference of cultures] wasn't a huge issue although there is obvious difference in idiom, in language but that didn't present any barriers." (Respondent 23)

According to one of the interviewees, the participants of cross-cultural videoconferences - native English speakers as well as those whose first language is not English - got used to translation-mediated communication.

"They were skilled themselves being in a translation situation because that was the reality of their lives anyway with their interactions, with English as a predominant language and there were people that were more used to an international stage, I guess. They were very comfortable in a translation situation." (Respondent 21)

Translation was difficult no matter if it occurred face-to-face or via videoconferencing technology, commented one of the respondents. He thought that a translator who can comfortably deal with a generic discussion will probably stumble if technical concepts were discussed.

"Translation is a difficult art. And simultaneous translation is even harder. I don't think it makes much difference whether it's videoconference or not, it's just difficult." (Respondent 24)

The above respondent's work involved extensive travel and frequent engagement in translation-mediated communication that normally occurred face-to-face but also took place via video-channel. Based on that experience, he developed a rather doubtful attitude towards the translators which he explained as follows:

“The problem always with interpreters is you're never quite sure whether they're interpreting you correctly. And the more technical a discussion, the less sure you are." (Respondent 24)

Another interviewee also supported the idea of language barriers being an issue, no matter what medium was involved. But at the same time, he implied 
that one of the sites using simultaneous interpretation had an affect on other sites.

“Timor [was] using a simultaneous translation and it did pose some... slight difficulties... I think that's just an unfortunate reality of the language barrier ... I think that would happen in any meeting whether that was face-to-face or a videoconference." (Respondent 19)

Two respondents who attended the same videoconference on anti-corruption were unaware of the fact that simultaneous interpretation was used at one of the participating sites. They were quite surprised to find out that it was a translator's voice that they heard from Timor Leste.

"I didn't think they did [use simultaneous interpretation]. I thought everyone spoke in English... Oh, ok. There was someone translating! ...Well, then it's done well because I didn't notice it [laughs]." (Respondent 23)

However, Respondent 19 who attended the sessions on fisheries management topic was quite unhappy about the quality of translation that was coming from one of the participating sites. He implied that poor translation was made worse by audio problems.

"In this particular case the translator himself was extremely unclear. It was physically difficult to hear him and then even if you could hear him, often the words simply didn't make sense. So, I know that the Australian end, a couple of times, had to ask him "could you repeat that, we didn't really hear what you said". (Respondent 19)

The above quote suggests that there were times when a moderator in Canberra simply did not understand what the translator was saying but being polite he used audio problems as an excuse when asking the translator to restate the phrase.

A similar situation that took place during videoconferences on anti-corruption topic was perceived by another participant from a different angle. He pointed out that the translator had problems coping with the pace of the discussion and the moderator's speech in particular. The theme related to native English 
speakers talking too fast and posing problems for translation-mediated communication. This has been brought up in Australian and Russian cases as well.

"I know that each time there was a request ...for the ANU professor who was chairing the conference to speak slowly. And perhaps, pause every now and then, which I think worked in theory but I just felt sorry for some of the people particularly in Timor who, had to stop on a number of occasions and ask that the discussions be slowed down a little bit." (Respondent 19)

Translator's requesting the speakers to slow down and moderator's asking the translators to repeat what they said was rather time-consuming, according to one of the respondents. He suggested that the videoconferencing channel could have been a factor but he was confident that there must be ways to overcome this challenge.

"It's just a result of the requirements of the translator. They've got us actually to repeat everything twice, or four times if back and forth... and that was I guess, exaggerated a little bit because of the videoconferencing, but ... Yeah, that was another challenge but definitely not something that couldn't have been overcome." (Respondent 19)

To sum up, the respondents in the New Zealand case who, with one exception, were native English speakers, agreed that cultural differences of participants definitely present some language barriers. At the same time, they pointed out that using English as a language for communication during videoconferences was the right choice because it was a first or official language in most of the participating countries. One of respondents got an impression that some participants were quite used to engaging in translation-mediated communication. A couple of interviewees thought that translation was a difficult task no matter what communication medium was involved. At the same time, there were people who felt that the videoconferencing channel might have been a factor in making translation even harder. The fact that one of the participating sites used simultaneous interpretation was unnoticed by some interviewees whereas others knew it but felt that no problems were presented 
because of that. The issue of presenters talking too fast was brought up again as in two previous cases. That made the translator's task more difficult and consumed much time. In an unrelated case, the quality of translation was so poor that the participants did not understand what the translator was trying to say.

\section{Facilitation and moderation issues}

Respondents in the NZ case as well as respondents in previous two cases used terms 'facilitator' and 'moderator' interchangeably because they thought that the roles are quite similar. All respondents recalled receiving an email informing them about, and inviting them to participate in, a videoconference. They understood that the person from VUW who sent the invitations and communicated regarding the event was a facilitator for the videoconference. One of the respondents - a VUW faculty member -did not even know that the university had videoconferencing facilities before receiving an email. Another respondent - a government official - was the only participant from a NZ site in a videoconference that discussed infrastructure financing issues with a number of other sites. He recalled that towards the end of the session the NZ site had been cut off for no apparent reason. Having waited for a few minutes feeling awkward on his own in a videoconferencing room, he had left.

"I did get an invitation to go to the next topic but I was never told. I never found out whether the whole system had crashed or just the New Zealand end of it and yeah, there was just no follow up at all. So I guess that wasn't very well handled and I didn't - for other reasons - go to the second one ...

Not seeing what the end of it was, if there was an end, made it much more limited value." (Respondent 20)

The respondent above assumed that the system had crashed or there was some kind of technical problem that caused the disconnection of the NZ site. But the reality was, according to a VUW videoconference suite technician, that the NZ site got automatically cut off when the time - usually 2 hours - booked with the World Bank's Network Operations Centre for bridging services, was up. Nevertheless, the fact that one of the participating sites had been cut off without 
warning when the session was yet to be officially adjourned, left a participant with a sense of incompleteness and contributed to his decision to decline an invitation to the next event.

It is evident that no one - neither a facilitator nor a technician - was available to provide explanations to this participant. However a few other interviewees mentioned that the VUW person, who had initially contacted them regarding the videoconference, greeted the participants in the videoconferencing suite, briefed them about the event, distributed the handouts and left the room shortly after the start of a videoconference.

"Aside from getting us to attend, [she] basically just provided an overview of what we were there for, of what's going on... it was reasonably minimal, the input. But I didn't feel like that was something that we needed to be walked through necessarily. It was relatively self-explanatory. I think she left during the actual discussions and then returned at the end." (Respondent 22)

Another interviewee who participated in some videoconferences from a series on anti-corruption strategies also mentioned that the technician, as well as the facilitator, was not around when these videoconferences took place. While mentioning that, he acknowledged that there was no need for anyone to be present because the videoconferences went on smoothly in terms of technical set up and functionality. This respondent also recognized that the facilitator was the person who made possible his participation in this event.

“There wasn't anyone on site at the time dealing with things from a technical point of view ... That was set up before we moved into the sessions and there were no problems. There didn't really need to be anyone there. [Name of facilitator] didn't sit in on our discussions herself but my understanding is she was the centre of organizing it, our participation in that particular conference." (Respondent 23)

Normally, a technician was always on standby when a videoconference is on that was the case during the events that were observed during the research fieldwork. 
One respondent participated in the first session of the series of videoconferences on the topic "Entrepreneurship and small business development in the Pacific region" alongside the prominent representatives from P.A.C.I.F.I.C.A. Inc., a national NGO for Pacific women living in $\mathrm{New}$ Zealand, and the School of Maori Business at VUW. Based on this experience, she realised that such an event potentially could have had more impact if organised differently:

“... the conversation was facilitated by white Australians and there was limited opportunity for real dialogue... I think that whilst those same people will still be interested in being involved again, they would insist on being involved in a different kind of event ..." (Respondent 21)

This informant implied that such an event should have been facilitated by someone from the region, preferably a native of one of the participating Pacific countries. In this quote she referred to a person chairing a videoconference as a 'facilitator'.

A respondent, who attended all four session of the series of videoconferences on fisheries management topic, got an impression that the organisers had problems fitting the content into the time allocated for each videoconference:

"The material spilled over from one week to the next... So it may be the facilitators were not realistic in the expectation of what could be covered in the two-hour slots that they had. It could be that there was more discussion than they expected." (Respondent 19)

He clearly remembered that towards the end of the last session the moderator rushed through the remaining topics on the agenda so that they were not covered to the extent intended. Based on that he concluded:

"I think it was well structured, but couldn't fit in the time available. So one of two things is true: either there wasn't enough time allotted, or the structure should have been changed to fit the time that was available." (Respondent 19) 
In contrast, the videoconference on the infrastructure financing topic had the same two-hour time allocation but very few people were willing to talk. A participant of this event noted that despite the moderator's attempts to encourage a dialogue, most of the participants preferred to remain silent.

“He [moderator] asked questions and invited some of the people to speak ... most of the other ones actually either declined to speak or said very little. So the other Australian person and myself ended up doing most of the talking just because not a lot was being said." (Respondent 20)

At the same time, this was the session during which the NZ site had been cut off before if had been officially concluded by a moderator. It suggested that this session did not fit in the allocated time despite the lack of lively discussions. In the sub-section discussing cultural differences in the NZ case, this respondent proposed that one of the possible reasons for people not saying much was a gap in the level of knowledge and experience on the topic. On the other hand, participants might have preferred not to speak because "there is an unintended conspiracy of courtesy on the part of local nationals, preventing crucial communication between them and alien experts from occurring freely" (Ascroft, 2006).

Talking about the role of a moderator during videoconferences, one of the interviewees referred to a person chairing a meeting or coordinating a training sessions in a face-to-face setting. She summed up by stating that the roles are similar because in all cases the moderator aims to meet the objectives that have been set for that particular activity, no matter whether it was taking place via video channel or in face-to-face mode.

"It relates to what the bigger picture is and what this event is for - comes back to the objectives really, and it's up to the moderator to ensure that those objectives are fulfilled so it comes back to the whole issue of educational design or communication design." (Respondent 21)

A different respondent also suggested that moderating a videoconference is not very different from chairing a face-to-face meeting. He actually praised the performance of the person who moderated all videoconferences in anti- 
corruption series from Canberra for a job well done and compared it with some other face-to-face meetings on his memory that were much less effective.

"I think it [moderation] is very important, in the same way as with any meeting. It always works more effectively, more efficiently when there is a chair, someone directing conversations, moving topics along... And it worked very well... I've been to meetings that have been a lot more disjointed and a lot less well run. So in that sense, it was quite efficient." (Respondent 22)

One of the interviewees pointed out that he was able to see only one site at a time - the one where someone was talking - so he spoke only if he was given a floor by a moderator. Therefore, he felt that without someone managing the floor, a video-mediated discussion would not have been achievable.

"It was necessary for one person to act as a leader and lead the discussion and provide an introduction and then actually act as a traffic policeman and invite people to speak and then switch to another person so without the moderator it would have been impossible." (Respondent 20)

Another informant pointed out that the moderator's microphone was always on so he was the only person who could be heard in parallel with someone else talking. According to this interviewee, that allowed a moderator to chair the session more effectively by making comments when necessary or interrupting someone if need be. This informant realised that a video channel actually enabled more efficient floor management compared to face-to-face setting because during videoconferences people could be heard only if they were given the floor by a moderator whereas during a face-to-face meeting several participants could talk at the same time.

"He [moderator] was able to speak at all times, which was good, because you need someone to be able to interject and say "ok, that's a good point you've made ... ok we move onto the next thing" which worked really well. And in a sense it was better than a normal meeting because ... to make a counter-point you had to wait for your turn which was interesting and everyone behaved, which was good. It worked very well." (Respondent 22) 
Someone else who attended two sessions from the same series on anticorruption policies also thought that they were moderated well. He explained that the sites were given some time to discuss a question after which they were given the floor in turns. The observation notes confirm that the moderator called in the sites in alphabetical order of countries names, sometimes in a reverse order so each site had a turn but in different order - a common approach in GDLN context.

"He [moderator] held it well together. He had prepared a list of topics and questions for each of us, each group to consider and we rotated around the groups ... There was also an opportunity to develop the conversation depending on how and what the responses were. So it wasn't simply too tightly structured and I think that particular conference seemed to strike a nice balance." (Respondent 23)

Respondent 22 recalled that the first session of the anti-corruption series of videoconferences began with a brief self-introduction of every participant at each connected site. He felt that it was a time-consuming and unnecessary exercise that used up limited time that could have been used instead for discussions. He suggested that if only a small number of active discussants introduced themselves and the others were just observers, the allocated time could have been used more productively.

"Perhaps if you had maybe two, three, maybe five max, people who're actually speaking, and as many observers as you want ... I think then things would've flowed a bit better and maybe [use of time] would've been more efficient. Maybe just having spokesmen for groups, would perhaps have worked better." (Respondent 22)

This respondent explained that people whom he attended the videoconferences with organised themselves better from the second session on by agreeing to speak in turns and presenting the view of their group each time the NZ site was given the floor. He emphasised that all participants from the NZ site had a chance to speak on behalf of the group incorporating ideas and comments expressed by each individual member. 
"We just had a panel of about 5 or 6; there was no sort of organized hierarchy amongst us. It was just a discussion amongst officials. And I think at the second one we decided that we would discuss and then someone would be a spokesperson from the group. And each time we just moved that along so someone else spoke. Which seemed to work quite well. I think it worked fine, just having one, just a single moderator or lead moderator back in Canberra." (Respondent 22)

The observation notes suggested that during the first session each participant in the NZ site presented his/her opinion one after another every time the site was given the floor. However they had altered this practice from the second session on by nominating one speaker that would express the consolidated view of participants at the NZ site and that allowed for more productive use of videoconference time at their end. Based on experience from the first session, the participants had a chance to change the tactics to improve time management for subsequent videoconferences because the event consisted of four weekly sessions. The participants would not have had such an opportunity if the event was just a one off two-hour videoconference.

The moderator's approach of allowing each site to discuss an issue before opening the floor was well received by another participant of this event. He benefited not only from sharing ideas with participants at other sites but also from exchanging views with other people in the same room representing different stakeholder groups in NZ: government, academia and NGOs.

"We were able to quickly share ideas. None of us had done any real preparation. We were only drawing upon our background knowledge. But being part of that conference wasn't just about talking to people at the other centres but also talking amongst ourselves in preparation to talking to other groups." (Respondent 23)

This respondent was confident that the moderator's performance enabled a productive discussion and he doubted that a different approach could have been better: 
"His moderation was important. I mean, had it not been structured like that, who knows, it might not have been as effective as it was." (Respondent 23)

Moderation will improve the efficiency of any video-mediated meeting, thought one of respondents. He reflected on some World Bank operational videoconferences that he attended in the past - some were moderated and some were not - and concluded that those with a moderator that followed a pre-set agenda had been more efficient than unstructured group discussions.

"I think those discussions are of value if more structured... because the time is limited. And some people, it doesn't matter what culture they come from, feel more comfortable speaking at a videoconference than others and they [the latter] need some support." (Respondent 24)

In summary, all respondents in the NZ agreed that moderation plays an important role in the effectiveness of videoconferences. Moderators' approach to managing the floor and time had direct implications to perceptions of interviewees about videoconferences. Some events were moderated better than others and thus left different impressions. One respondent suggested that moderating a video-mediated discussion was easier than chairing a face-to-face meeting. In the first case a moderator has greater control in managing the floor by muting and un-muting the microphones. A few respondents likened moderating of videoconferences to chairing face-to-face meetings or training sessions. They suggested that no matter what the medium was, every communication aims at meeting the pre-set objectives and moderators can significantly contribute to the mission.

According to the participants, facilitation of videoconferences primarily consisted of email communication prior to the event, minimal input during the videoconference and zero interaction after the session. A couple of respondents acknowledged that the presence of a facilitator or IT technician was unnecessary because the videoconferences went on without any hitches. However, one of the participants felt confused when he firstly realised that he was the sole participant attending the multi-site videoconference from the NZ end, and secondly, when the connection was cut off all of a sudden. He implied 
that having anyone around - a facilitator or a technician - would have been beneficial for understanding the situation.

\section{Presentation issues}

Different presentation styles can have varying implications for the audience, said one of respondents and illustrated his point by an example. The videoconferences he referred to were multi-site sessions from the series on fisheries management in the Pacific region and one of the participating countries, East Timor, used simultaneous interpretation.

"On the fisheries one, there were ... two main presenters; they had two distinctly different styles. ...one where he was too passionate about the subject and spoke very quickly, pushed a lot of information out there at very, very high speed. And unless you were moderately well versed in fisheries management, you actually may have had trouble following that. The other person, [name] I think it was that I actually really, really liked. I suspect he is a fantastic teacher. Explained stuff, I thought, very, very carefully, very clearly." (Respondent 19)

In the above quote, this interviewee, a native English speaker, emphasised that one of the presenters spoke at a very fast pace even for people who know the topic well. His complains about poor quality of interpretation during this event mentioned in an earlier sub-section discussing language differences and translation issues. A link between the two comments from Respondent 19, one about the fast-talking presenter and the other criticising the quality of interpretation is evident. Simultaneous interpretation of communication on such a complex topic as fisheries management could have been made even more challenging if one of the presenters was talking very fast.

A participant of the videoconference on infrastructure management positively commented on the communication skills of the presenter:

"He made an introduction and he spoke clearly. He laid out issues for the other participant to speak about. He did his job very well." (Respondent 20) 
Another interviewee thought that presenting at a videoconference is not different from presenting in a face-to-face environment. She suggested that the core of success in any medium depends on the level of a person's communication skills and prior experience.

“Those people who are good at it do it not because organisations help them to get good at that but because they're good communicators. Because they understand cross-cultural communication by themselves anyway.... naturally or they've got experience elsewhere." (Respondent 21)

In contrast, a couple of interviewees agreed that presenting at a videoconference is harder than talking to a live audience. In justification of this opinion they mentioned lack of eye contact, limited opportunity to feel the audience and receive immediate feedback, the varying quality of video and grasp of the camera, etc:

"Probably it's a bit harder to see how your audience is reacting. And a good presenter should always be aware of that. It may even be harder to pick up when they want to ask a question, interrupt you or something because of the quality of the feed and the way it's done... generally the quality of the picture is not always high and ... you couldn't even see if somebody is falling asleep..." (Respondent 24)

"From memory for example, you got images of the members of about four different groups in front of you at any one time. So that fact alone makes it hard to get that sort of feedback which comes from [what] you would call 'waves in the atmosphere' where you are in a face-to-face encounter in a particular venue." (Respondent 23)

Nevertheless, one of the above interviewees suggested a possibility for reducing such barriers by the speaker announcing some rules for interaction before the actual presentation starts and agreeing on possible ways for members of the audience at participating sites to provide feedback or seek clarification during the talk.

"Maybe better if you set some ground rules at the start "...I can see you right there [so] please, if you want me to stop because there is something 
you're not following ...because I may not otherwise notice", something like that..." (Respondent 24)

The objectives of communication should determine the presentation style, stated one interviewee. She explained her view by differentiating two different objectives, the less interactive one was a mere delivery of information and knowledge from the speaker to the participants and the other, more interactive way of communication was where the participants aim to exchange knowledge and experience.

"That would depend on what the objectives of the session were, so I think that the role of the presenter could be a number of different things... Presenters whose role it is to impart information, give a lecture ... But in fact that it is largely a one way street but with some questions where the subject experts are giving their knowledge. But you can also have presenters that run a more facilitative style and that is a purpose of the exercise, the blending of more information expertise from the participants..." (Respondent 21)

She underlined that different qualities are required from the presenter. The aims of communication and that the role of a presenter depend on the communication design. According to her, a knowledge sharing exercise will require more skills from the presenter compared to a traditional training session or lecture.

"Situations where you recognise the significant expertise in each of the participating groups and part of the purpose is to bring out all the skills and all the knowledge so that it becomes pooled and shared knowledge and that everybody is learning from one another... The role of a presenter then requires very strong facilitation skills to ensure that all the participants are making all the best participation that they can at all the opportunities." (Respondent 21)

The perceptions of interviewees in the NZ case regarding presentation issues can be summarised as follows: the presenter's role is different depending on the objectives of communication. Also, the presentation style of the speaker - pace 
of speech, approach to delivering the information and so on - has an implication for the participants' engagement and consequently, for the effectiveness of communication. A couple of interviewees felt that presenting at a videoconference is more difficult than presenting in front of a live audience, but one respondent was confident that there is no difference. 


\section{4. The Mongolian Case}

The fieldwork for the Mongolia case was carried out in August-October 2007 in Ulaanbaatar, Mongolia. The data gathered includes notes taken during three videoconferences observed and interviews with nine participants of videoconferences who were representatives of the private sector, nongovernmental organisations, international development institutions and academia. All interviewees were Mongolian nationals - men and women aged from 25 to late 40's - educated in Mongolia, Russia, Europe and the US. They participated in videoconferences with different roles. Some facilitated and/or moderated the sessions, some made presentations whereas others attended videoconferences as regular participants. Two interviewees participated in videoconferences as simultaneous translators. Respondents' videoconferencing experience ranged from just one session to numerous events - specific project meetings and more general one-off or a series of discussions - that took place via videoconferencing technology. All participants had varied experience of face-to-face cross-cultural interaction and a few people had participated in audio-conferencing discussions involving people from different countries.

\section{Cultural differences of participants}

The videoconferencing experience of interviewees in the Mongolian case consisted of events ranging from training courses, policy dialogues, project follow-up meetings, video-lectures and media-launches of the World Bank's flagship publications. Some activities, especially training courses, consisted of several video-sessions but the majority of videoconferences was just a one-off event. Seven interviewees had extensive experience of participating in various videoconferences over a numbers of years and two people had participated in just one videoconferencing event.

Most respondents emphasised that most of the events in which they took part were more of a one-way information delivery exercise than an interactive knowledge and experience sharing activity. Speakers at one site were 
presenting and participants at other sites receiving the information. During such videoconferences the audiences from content-receiving countries conversed with the content-providing site only but not with other participating sites. Two interviewees specifically mentioned that the communication during the Question \& Answer sessions was not an interactive discussion either because participants asked questions only to clarify or verify specific aspects of newly-acquired information. Below is how the respondents explained this situation:

"People in Washington made a presentation to four countries at the same time. People in each country communicated with Washington only... For example, we only communicated with the people from America who worked on "Doing Business"4 [report] but not with people from India, Indonesia, Vietnam." (Respondent 26)

"It's one-way information delivery rather than a dialogue. It's still one-way communication even if questions are asked... It's like: there are some knowledgeable people; they are dispensing their knowledge; the knowledge is received here; unclear aspects are clarified. I wouldn't say it's an exchange." (Respondent 29)

Based on her observation of the way people from different countries were conversing with the Washington-based presenters, Respondent 26 concluded that the cultural differences of participants had no major influence on the communication process:

"I noticed no significant differences watching people from each country communicating with the Washington office. Cultural differences cannot be seen if they can clearly articulate their questions in English and people understand them... Because the discussion topic is the same, all participate in the same event." (Respondent 26)

\footnotetext{
${ }^{4}$ Series of annual reports comparing business regulations in 181 economies (http://www.doingbusiness.org)
} 
Respondent 33 thought that people in general tend to control themselves more when attending in videoconferences so their cultural differences are not so obvious.

"For example we say that the Italians are wordy, the Asians are like this and the Germans are like that, etc. But [in a videoconference] the variation of these differences is relatively low. Because I think that on the screen, in the camera, people control themselves more." (Respondent 33)

Another interviewee who participated in videoconferences as a simultaneous interpreter, also thought that the difference of cultures did not have a significant effect on communication. However, she observed that differences in terms of a country's experience influenced the communication more than differences in terms of national culture. She explained that normally, the participating countries discuss a particular topic that is relevant to all of them, but each country's experience on the subject is specific.

"I think, experiences of different cultures rather than cultural differences will have an impact [on communication]. The differences [of cultures] will not be evident because everybody comes with one mind to get the best solution for the particular issue which they are talking about. And then, they share their country's experiences and practices. That is more evident, I think [during videoconferences]." (Respondent 30)

She then explained that most videoconferences she translated for were on policy-related issues when professionals from a number of countries, some developed and some developing, gathered together with good will to discuss various topics on development. Therefore, according to her, cultural differences of the participants do not interfere in this type of communication.

"Participants are very serious people. They come to help each other, not to compete. They discuss just regular policy-related issues, not conflicts, and therefore they have never demonstrated any cultural differences, something like 'I'm from a superior nation, you're from a poor nation' and so on." (Respondent 30) 
Respondent 31 also thought that in cross-cultural communication differences between countries' experiences mattered more than differences in their cultures. While attending a training course on food safety issues he realised that the participating countries differ in many aspects of life:

"For example, food production methods in India and Nepal are different. They have small farms or large automated livestock farms... We are different. We have a nomadic culture [pastoral husbandry]. For example, in the mountains one household with 500 sheep, the other with less than $100 \ldots$ We manage food production and food safety very differently. We can't adopt food safety systems that work for large farms and plants. So there are huge differences in this regard." (Respondent 31)

According to the above interviewee, the participants of videoconferences understood these differences well and learned from each other's practices and experiences. Participants' knowledge on a particular subject expanded as countries shared their experiences. Respondent 31 thought that, people reflected on these differences, compared themselves with others and thought creatively,

“Most importantly, people understand these differences and express their ideas on the issue based on this understanding. How can we implement it in our country, what opportunities do we have? From this perspective, differences of cultures are beneficial." (Respondent 31)

Countries involved in video-mediated cross-cultural communication of a training nature can participate as either content-providers or content-recipients. In the experience of Respondent 27, the content-receiving sites in videoconferences were usually Asian countries whereas the trainers or presenters were from the United States or Europe.

"In those videoconferences I attended, the majority of participants were Asians. So the recipients were mainly homogenous but the providers were usually Europeans or Americans and they would deliver all the information." (Respondent 27) 
Some Asian countries provided content as well. Two interviewees participated in the same series of videoconferences on ISO quality standards where the content was developed by the Asian Productivity Organisation (APO) based in Tokyo, Japan and the trainers were Malaysian specialists who were linked to the videoconference from a DLC in Kuala Lumpur. In the case of another videoconference on Toyota Production System observed during the fieldwork, the trainers were Japanese and they presented from the Tokyo DLC.

One of the respondents who participated in a series of WB-initiated videoconferences on infrastructure project financing noticed that the audiences at participating sites - countries in East Asia - engaged in Q\&A sessions differently. He got the impression that in some countries certain people were assigned in advance to ask questions whereas in other countries the participants acted spontaneously and just spoke to the microphone if they had a question or a comment.

“As I remember, there were 30-40 participants in Vietnam but just a couple of them asked questions, it looked as if it was organised that way. Whereas from Singapore or Philippines, there were very few participants, just 5 people present, but all of them participated very actively." (Respondent 25)

The way participants in Vietnam organised themselves could have been due to a large number of participants compared to other participating sites. According to the above interviewee, the Mongolian participants - about 20 people and mostly government officials - were not particularly active but he noticed that women were more outspoken than men.

"Level of involvement from the Mongolian side was relatively low... women were more active whereas men preferred to listen." (Respondent 25)

The following comment by Respondent 30 on the difference in attitudes was associated with the various organisational cultures of the participants. She thought that government officials were more interested to learn from other countries' practices as well as share their own experiences. NGO representatives preferred to listen whereas staff of international organisations were more outspoken. 
“For example, representatives of international organisations are ready to share. They like to talk.... NGOs have a "I don't know much, so I'll just listen" approach. Government organisations' positions are "we hear that you do that; we want to do this" rather than cultural." (Respondent 30)

Another respondent also agreed that staff of international organisations tended to talk more but she thought that World Bank staff pushed through their ideas and dominated the communication. This referred to differences of organisational cultures and the power distance dimension of culture (Hofstede, 2001).

“Power difference was obvious - that's also related to cross-cultural [communication] - the WB experts have a tendency to guide, direct and tell what they have to tell." (Respondent 29)

Based on her experience acting as a local trainer for an online blended course that was using GDLN facilities for videoconference component of the program, Respondent 27 observed that participants in Asian countries displayed different attitudes towards the learning process. She compared participants from participating countries and concluded that they differed in terms of organisation, attitude and communication.

"Mongolians generally are not very well-organized learners in terms of time. They think that they can catch up later, read everything at once later on... Cambodians, Vietnamese, Chinese read a lot. They make efforts and that can be seen. They ask detailed, technical questions. Indians, Bangladeshi and Pakistani people have good English skills.... they talk a lot, make it too broad when asking questions, I think. they like engaging in debates." (Respondent 27)

Another respondent who acted as a local facilitator for a series of APO training courses thought that when people from different countries regularly communicated on particular topics, they started to speak a common professional language no matter what national culture they came from. In other words, they share the same organisational culture because they all work in the 
same field and pursue the same goals as members of the regional umbrella organization.

“Then all our NPOs [National Productivity Organisations] become like one family. And the topics are all relevant to national productivity organisations. We specialise in particular topics so we almost speak the same language. Therefore, there are no differences between us." (Respondent 28)

The above interviewee also thought that Asian countries had lots in common in terms of culture. According to her, cultural differences were barely noticeable when people from several Asian countries participated together in a videomediated training course.

"In general, the Asians have similar emotions, resemblance in terms of culture. Perhaps if we were mixed with the Americans or the Europeans then probably [cultural differences] would have been more evident. But we normally do [training courses] with Cambodians, Vietnamese, Pakistanis. So, no big differences can be seen." (Respondent 28)

The countries she mentioned belong to different sub-regions of the Asian continent: Vietnam in East Asia, Pakistan in South Asia and Cambodia in Southeast Asia whereas Mongolia is considered to be a part of either East Asia or Central Asia.

In contrast, another interviewee thought that in Mongolia, people have many different sub-cultures although they all belonged to the same national culture.

"Mongolians are a people with a very hybrid culture..., there are many cultures. For example during the last videoconference ... an officer from the Open Society Forum ${ }^{5}$ had been more active because it's a very globalised NGO... But there are also people who went through Mongolian schooling, had been in the past and continued to be 'cooked' in the Mongolian culture. It is very difficult for such people to express themselves." (Respondent 29)

\footnotetext{
${ }^{5}$ Mongolian Foundation for Open Society (http://www.forum.mn), affiliated to the Open Society Institute created by George Soros.
} 
By saying 'people who went through Mongolian schooling' this interviewee contrasted them with other people who went through alternative schooling. These were the individuals, including the interviewee and myself, who went to one of about twenty Russian secondary schools that operated in Ulaanbaatar and in other major cities back in the 1970s, '80s and '90s. For comparison, there is now only one Russian school operating in Ulaanbaatar under the auspices of the Russian Embassy in Mongolia.

Respondent 29 then explained why she thought that many Mongolians are so reserved and restrained. According to her, the socialist system that existed in Mongolia for over seven decades, shaped a certain mentality that prevented people from openly expressing their ideas and opinions.

"I think it's not because of them, it's because of our socialist legacy.... when instead of "you have a different opinion", they would say "you are wrong." And then, people were so scared to be 'wrong', they got a dichotomous, black and white frame of mind. And that impedes [communication] a lot, I think." (Respondent 29)

Being a Mongolian national, I fully agree with the above proposition.

A series of policy dialogues on Avian Influenza, training courses on microfinancing, productivity, quality assurance, infrastructure financing and other topics consisted of four to five videoconferences. A number of interviewees who participated in some of these events experienced the participants getting used to each other and the videoconference atmosphere getting less official. Participants of these events explained their experiences as follows:

"People opened up more. At first, to "do you have questions?" most countries would say "no questions, thanks". Towards the end [participants] got focused. Maybe their understanding of the course improved, so they opened up." (Respondent 32)

“They meet like friends. ... someone's son was ill, so next time others would ask about his health... or "last time you were very busy with a conference, did it go well?" and so on. Very personal relationship, very cute. Of course, 
if they come to Mongolia, the [relationship] will get even better." (Respondent 30)

Respondent 31 thought that when the same group of people met several times over videoconferences, they observed each other, got certain impressions, made assumptions and drew conclusions about each other. Observing each other's behaviour and communication, the participants learned lessons, positive or negative.

"It's interesting to watch how people communicate. Some are polite whereas some laugh offhandedly and behave in "that's us" manner... Some people set good examples but watching others you get the impression "this is wrong, you shouldn't do that". For example, the Nepalese laughed a lot, behaved frivolously, sometimes openly poked fun at others..." (Respondent

This interviewee thought that participants of training sessions conveyed through videoconferencing technology should take the event seriously and act formally. Another interviewee who also participated in the same course, also commented on Nepal's participation in the videoconferences. She thought that their light-hearted attitude affected the effectiveness of the event from a time management perspective.

"[People in] some countries, for example Nepal, laughed a lot and made jokes thus wasted time. A 10-minute Q\&A session would take 20 minutes because of their laughing... Time management was not to my expectations." (Respondent 32)

Many Mongolians would think like Respondents 31 and 32, believed another interviewee. According to her, Mongolian people have two distinct behavioural and communicational attitudes in life: formal and informal.

"In a formal setting people behave themselves very differently than in an informal - a huge gap! For example, when we conduct training [face-to-face] we try to make people feel comfortable. We laugh, say silly things because you're telling people "it's ok to be not so smart, it's ok if you say something silly." Only then people will participate [actively]." (Respondent 29) 
She thought that by establishing a cheerful atmosphere the organisers could achieve improved participation and communication whereas Respondent 31 thought that such behaviour was inappropriate for a training course.

In the perception of Respondent 29, Mongolian people became very timid in a formal setting, almost unwittingly controlling themselves and acting in a manner they think is adequate for the situation.

"Unconsciously, they think: "Oh, I'm now in the World Bank office. What if I stumble, say something wrong or look foolish?" They don't even realise [that they think that way]! Because Mongolians are not aggressive in such a setting, they can't feel comfortable and don't express straight away what they want to say." (Respondent 29)

An interviewee who often facilitates and moderates videoconferences thought that people in general, not only Mongolians, act differently than their usual selves when they attended videoconferences.

"In general, in other regions as well..., people become more controlled when they come to a videoconference... restrained rather than interactive. In general, no matter what culture they come from, people tend to control themselves more and are incapable of playing a part in an interactive show. They just stare at the screens, participate in a 'cool' manner." (Respondent 33)

Respondent 33 thought that a participant's personality plays a big role in the training process, regardless of cultural differences. An introverted person, no matter from what country, will learn a bit slower than an extrovert.

"In a training [course], some people are able to learn quickly and effectively. Someone who is open will get it fast. But if a person is a bit shy, it will take time. Because it's the same, during a face-to-face meeting he will be shy, too." (Respondent 33)

In summary, the majority of respondents in the Mongolian case agreed that cultural differences of participants- on a national and organisational level influenced the cross-cultural communication that takes place through 
videoconferencing technology. A number of interviewees thought that national cultural differences have no significant influence on communication but provided different explanations. Some thought that cultural differences were hardly noticeable when people take part in a one-way communication. But others believed that participants were apprehended by the technology and official setting thus controlling themselves more. A number of interviewees thought that differences in the experiences and practices of participating countries affected the communication more than cultural differences. A few other respondents noticed that the participants differed more in terms of organisational culture rather than national culture. As perceived by some informants, various attitudes displayed by videoconference participants were influenced by the national cultures they belong to or by people's individual personality traits. Several interviewees talked about the specific characteristics of Mongolians: their approach to a learning process, communication and behaviour in various setting and their common mentality. One respondent thought that the communist regime left a strong influence on the mindset of Mongolian people. A couple of interviewees believed that Mongolians were just like other Asian people. In contrast, another respondent was confident that Mongolian culture was unique and complex.

\section{Language differences and translation issues}

All interviewees agreed that one of the main issues faced by Mongolians participating in cross-cultural communication was related to the English language barrier. According to some respondents, Mongolian participants were disadvantaged compared to native English speakers because they could not fully express themselves and share their expertise with other participants.

"Native speakers have advantage because they speak in their mother tongue. They talk clearly and briefly. I noticed that non-native speakers may perhaps know the topic better than English or American people but they might express themselves a bit incompletely because they don't practice this language everyday." (Respondent 25) 
Another respondent thought that participants from countries where English was one of the official languages or was taught in schools as a first foreign language also had a plus compared to their Mongolian peers. Their vocabulary was rich so they could effectively communicate their ideas and feelings.

"We are the same but they have advantages in terms of [English] language. They can express, a gratitude for example, correctly and nicely whereas we just say 'thank you' because of language barrier." (Respondent 28)

Language differences played a role in cross-cultural communication, thought Respondent 26. Although her English was fluent, she found it difficult to understand accented English when attending various videoconference events.

"If more than 2 countries participate in one conference, their specifics will definitely influence [the effectiveness of the videoconference]. It will influence at least in terms of the clarity of the various accents of English people that are speaking." (Respondent 26)

To the ears of Mongolian interviewees, English spoken by participants from other countries had a different appeal: some pronunciations were easier to understand than others. Furthermore, the pace of speech was very important for grasping cross-cultural communication.

"I learnt that Cambodians have good language [skills]. People from Philippines speak very nicely, Pakistanis - very clear, Indians speak too fast... I have real trouble understanding people from Thailand and Taiwan." (Respondent 28)

"People from Philippines and Sri Lanka articulate very clearly. Indians and Nepalese speak so fast, difficult for people to catch up." (Respondent 31)

Another interviewee with fairly good English had problems understanding when people from other participating sites talked because each country spoke their version of English. She shared her experience of attending series of videoconferences as follows: 
"At the beginning we had a problem of accents [of English]. We couldn't catch at all. Towards the end however, our ears got used to these dialects." (Respondent 32)

The quote above suggested that she would have understood just a little if accented English was spoken during a one-off event because there would be no opportunity to get accustomed to hearing different accents.

Many Mongolians hesitated to speak English because they were afraid of being laughed at by Anglophones if they made mistakes. Videoconferencing technology reduced these concerns because of the remoteness of other participants, according to the following respondent:

"It was very difficult for me at first. I felt awkward because of the language barrier. But later on I got used to. Now I speak freely, I even think "they are far away, no one will make fun of me". I feel more confident than in a faceto-face [setting]." (Respondent 28)

Simultaneous interpretation during videoconferences helped participants to bridge language barriers. Although most participants with good English do not use these services and engaged in communication directly, they could still make assumptions about the quality of translation. An interviewee with fluent English explained as follows:

"I didn't listen to the translation but it looked like everything was followed well because people who didn't speak English would ask questions regarding the content in Mongolian. So it was clear that the content was delivered." (Respondent 25)

Another respondent, also fluent in English, thought that cross-cultural communication could never be translated in full. She was confident that even the best of translators could not convey the content in full and reproduce a presenter's emotions. On the other hand, she found translation to be distracting because she heard and understood both languages. 
"Translator cannot act exactly as a presenter. There is no way that a 100 percent translation can be achieved, no matter how good the translator is, maybe only 95 percent of content will be conveyed." (Respondent 26)

In contrast, Interviewee 27 thought that participants need not be fluent in English to understand each other if all of them work in the same sector and discuss sector-specific issues during a videoconference. She referred to the series of training courses on micro- financing delivered to several Asian countries.

"People who work in this industry generally speak the same language banking or micro-finance language - and for that you don't need to have [knowledge of] literary English. People in the industry normally have [English] language skills so there are no big language barriers for them." (Respondent 27)

Presenters at videoconferences expected the audience to have a certain level of specialised knowledge on the topic - knowledge which the interpreter would often not share (Shlesinger, 1995). Based on her previous experience in face-toface setting, Respondent 32 concluded that communication on industry-specific and technical subjects could be impeded by erroneous translation.

"During a similar course ... the terms used [by a translator] were totally incorrect but people with no English wouldn't even realise that they have been misinterpreted. They couldn't fully understand inaccurately translated terms. Thus, some translators can create misunderstanding." (Respondent 32)

On the other hand, she admitted that accurate interpretation of industryspecific professional terms should not be expected from generic translators. Even a specialist in the filed may not necessarily be able to know the correct translations of all terms, thought this interviewee.

"Very few people in Mongolia are fluent in English from a food industry perspective. And these people will never go to other organisations and work as translators... Of course, generic translators are unable to accurately interpret established professional terms." (Respondent 32) 
Accurate translation of professional terms was extremely important but a participant may not necessarily receive it every time, agreed another interviewee. He thought that translation during training courses helped participants to understand certain concepts.

"Translation is beneficial for participants from the point of view of understanding specific terms. However, the danger is that a lot depends on the translator. People won't get the main concept right if professional terms were interpreted erroneously. It's very risky." (Respondent 31)

Another interviewee thought that at times, translator's performance may be misjudged. She assumed that the participants would rather doubt the interpretation skills of their fellow Mongolian translator than question the competence of an international presenter if they didn't like what they heard.

"Often people complain about the quality of translation...On the other hand, they don't know what the actual presentation was like. In fact, it could be that a foreign presenter was talking too simplistically, didn't perform up to [participant's] expectations. The translator just interprets everything that the presenter says, but the participants will only suspect the translator. They may not even realise that the presentation was poor." (Respondent 26)

The quality of translation was important for cross-cultural communication, but it was not too difficult to provide, thought Respondent 33. According to her, the translator had a straightforward task: just to convert someone's talk into another language, as it is.

"Translator is just another profession. We are not asking her to elaborate on the topic. He/she just needs to properly interpret the expert's talk into Mongolian. It's very simple! No matter whether it's face-to-face or GDLN, just interpret well. That's all." (Respondent 33)

However, her following quote suggested that it is not that simple. Having facilitated many videoconference events, this interviewee acknowledged that a translator's performance would improve if she was adequately informed on the 
topic in advance. She was confident that the interpreters must be really good professionals in order to work for GDLN events.

"Of course, a lot will depend on an interpreter's performance, her skills. The background [materials] should be provided in advance, if possible. The translator should understand this background and the sector very well... Without doubt, you need to get good translators." (Respondent 33)

Two of the interviewees in the Mongolian case regularly acted as simultaneous interpreters for videoconference events although they had other full-time jobs and were not trained as professional conference interpreters. One of them confirmed that having some background information in advance was very helpful. Prior to each event, she proactively sought relevant information from organisers and participants.

“During the Avian [Influenza] Emergency Defence series... I knew nothing, so I used to come earlier and get some [printed] materials from people, ask them about things they want to talk about, try to get some content. Then I felt somewhat prepared." (Respondent 30)

The following quote by the other interpreter corresponds with the views expressed by respondents 26 and 31 in regard with participants' perception of translator's performance and skills. Confined in the translator's booth, an interpreter just focused on converting a message from one language to another and vice versa. She had no opportunity to observe participants' reactions and she rarely conversed with them.

"Because videoconferencing takes place in real time, with synchronous translation, there is no opportunity for people to clarify certain points that they've missed out. Therefore, it's difficult to know what was understood and what wasn't ... Of course, as a translator, I understand everything [laughs] but I don't know what the audience got, I don't know that." (Respondent 29)

On the other side, a translator can also miss out on something while simultaneously interpreting communication during videoconferences. In such cases, respondent 30 simply interrupted a speaker and asked for clarification. 
“I'm very straightforward: when I get lost, I just say "sorry, can you repeat that again?" because it's my obligation to make sure that [the participants] understand each other. Especially during videoconferences I do that because I want to make sure that all of them understood each other in just one-hour [event]. If I only cared about my own reputation, I would pretend [that I'm following] and go on..." (Respondent 30)

This interviewee thought that an interpreter should set aside personal ambitions and concentrate on her main task - helping people to communicate who come from different countries and backgrounds and speak different languages.

Her fellow interpreter thought that she could provide translation services effectively because her main job was based on interpersonal communication. She didn't merely translate words and sentences, she conveyed messages in the most understandable manner.

“Maybe because I don't only do interpretation, my job ... as an activist, is to make people understand things... When translating, I'm still doing that job, you see? To help people understand something. Sometimes, I disagree with that 'something' especially when they talk about neoliberal policy, etc... Why are they hiring me? They are hiring me to help people to understand something, so that's my job. And when it comes to understanding, it's not just about words, it's also about how you say those words." (Respondent 29) Both interpreters adopted different techniques in performing their tasks effectively. One of them tried to get as much relevant information prior to the event but the other one aimed to understand the core concept well and then pass across the translated version.

"I try to understand the main concept of what they are talking about. If the essence is clear, the details can be added later... Otherwise, sentence-bysentence translation is impossible, technically it's impossible." (Respondent 29) 
In doing so, the following respondent also strove to convey as much emotion as possible in parallel with the information. As they only can convey words, not body language, she used voice and intonation

"I think that the person who translates should be quite passionate. Otherwise, some translators speak very monotonously even when the presenter is quite expressive. Usually people are not very good in receiving monotonous [speech], a human brain easily switches off. So, that needs to be considered in [selection of] translators... In general, a monotonous interpreter may translate well but discourage people's interest." (Respondent 29)

In sum, all respondents believed that language differences impeded crosscultural communication to some degree. As the main language of GDLN videoconferences is English, communication is often mediated by translation. Mongolian participants with advanced English skills sometimes struggled to understand various accents spoken by their peers from other countries. The likelihood of misunderstanding was higher in one-off events than in a series of videoconferences because after some time, participants got used to each other's pronunciation and their listening comprehension improved. Discussion on quality and scope of translation was presented from two perspectives: participant's and interpreter's. The latter carry out their task of interpretation to the best of their skills and experiences that are often specific to their personal and professional qualities. Participants perceive the interpreter's performance based on the output - what they hear - rather than on input - what was originally said because they only hear the voice of an interpreter.

\section{Facilitation and moderation issues}

Mongolian interviewees had varying understanding about the role of a facilitator: some found it to be quite similar to a moderator's role, some perceived it only from the perspective of organising the logistics of the event, whereas others assigned complex content localisation or development functions to facilitators. The following interviewee believed that the facilitator should not be just sending emails about upcoming videoconferences to potential 
participants but making more significant contribution to the process of content selection and participant mobilisation:

"[The facilitator should] think: what specific audience to target in Mongolia?

Why [this content] is relevant to them? What questions will be raised? Is it useful for Mongolians? Should it be raised a bit more specifically, from Mongolian perspective?" (Respondent 33)

Respondent 29 had a similar opinion. In addition, she felt that the facilitator needs to engage with potential participants well in advance, convey their feedback to the content providers and recommend adjustments to the program:

"If something is going to be organised then [the facilitator should] find out things like who wants to participate, what they want to know, etc. and based on the audience's needs, profiles, interests and also after having shared the information about the people who'll participate from the other side, finally come up with the issues to be discussed." (Respondent 29)

Two of the respondents participated in videoconferences series of the Asian Productivity Organisation (APO) that were facilitated by a local NGO. One of them performed a role of a local coordinator and explained her duties, distinctive from that of a facilitator, as follows:

"The local coordinator is responsible for the papers and logistics, whereas the moderator/facilitator guides the group of participants from a professional point of view, responsible for linking up the case studies ... [the coordinator] takes care of organisational matters such as registering the participants, supplying them with [handout] materials, etc." (Respondent 28)

The other interviewee who took part in the same series of APO sessions thought that the local coordinator needs to carry out more important tasks such as closely coordinate with the moderator and communicate on behalf of the Mongolian group of participants.

"[The local coordinator] must establish a good rapport with the moderator, try to attract his attention to the team, present the team members or ask the 
fellow team members if they have questions, skilfully use the opportunity of the given floor each time the channel is switched to us." (Respondent 31)

A World Bank staff suggested that finding a good local partner organisation and delegating the task of videoconference facilitation had dual benefit: reduced her workload and strengthened the capacity of the national contractor that can reliably perform similar duties in the future.

"Or you can find a good partner that operates in that field, then it will carry out [the videoconferences], we just need to train them well. If you guide them once really well, then it will flow smoothly. Like 'the train has already moved', you don't need to go there every time." (Respondent 33)

One of the respondents implied that some preparatory work by a facilitator could contribute to the effectiveness of communication, suggesting that more communication and information sharing prior to the videoconference could be done online.

"As far as I know, [there was] no preparation. It might be different if ... some preparatory work is done, maybe online, depending on the audience... when someone knows in what [kind of] event he'll participate he'll certainly think about the topic, right? What do I want from it, what questions do I have in my mind, what do I want to find out, what do I want to share with others, and so on. That ... might give an opportunity for people to be more active." (Respondent 29)

Moderator's time keeping ability emerged as one of important factors when interviewees reported on their perceptions for the effectiveness of videoconferences. They believed that moderator should enable an equal, mutually interactive discussion by allowing adequate yet strictly limited time to all connected sites so that the audiences at participating countries can have an opportunity to express their views. Respondent 25 suggested that given the time restriction, the moderator should be able to carefully manage the communication by closely following the event's agenda.

"We had just two hours to cover two big topics, so it was very important to perfectly follow the running order. For example, just one country cannot 
keep talking because several [other] countries are connected, right? So it's important to enable equal participation." (Respondent 25)

The observation notes suggested that the role of a moderator was especially important during Q\&A sessions, because each site must be given a floor and treated equally and the many sites are connected, the more complicated the task is. The following quotes supported my observation.

"During various conferences the moderators perform a very important role, especially during Q\&A sessions." (Respondent 30)

"The most important [functions of] a moderator is to arrange and relate the questions, maintain the atmosphere in the audience." (Respondent 31)

In contrast, some respondents implied that the importance of moderator's role may vary depending on the type of the videoconference. For example, reflecting on her experience from moderating during a videoconference lecture of a prominent speaker from one location to another, Respondent 26 noted that her task was not too challenging because her involvement was required only during the Q\&A session:

“There wasn't much moderation required... nothing special from content or organisational or logistics point of view. It was so simple: just a lecture and a round of questions and answers - nothing too complex." (Respondent 26)

Another respondent suggested that not much moderation is required if a videoconference is a discussion on a very specific topic involving participants who already knew each other or professionals that work on the same project. According to her quote below, the members of such discussions all engaged in communication so that the moderator's involvement was minor.

“In those videoconferences the moderators didn't perform a very active role. The "avian flu" one was very specific and people just run it by themselves, it's like a meeting of members of the same team, so it's different." (Respondent 29)

An interviewee, who participated in videoconferences as a presenter, suggested that at the start of the videoconference the moderator should make some 
introductory comments highlighting the main aspects of the topic and introducing the presenter so that the participants at remote sites will have a common understanding about the objectives of the session as well as establish a prospect about the key speakers.

"I noticed that it's really nice if the moderator summarises in the beginning of a videoconference: what are the main areas to pay attention to, how the presentation is linked to the topic, why a particular presenter was invited, what is that person's experience and so on. A videoconference that was well moderated is more effective because some participants come to a videoconference without any background or preparation." (Respondent 27)

Observation notes suggested that in most cases, the moderators began the sessions by welcoming the attendees, explaining the goals of the session, introducing the presenters and announcing some basic 'house rules' and seeking cooperation from audiences at all participating sites.

Respondent 31 reflected on the moderator's communication and organisational skills and prised his ability to engage the audience, maintain participants' interest by slipping in occasional jokes and other improvisations to prevent boredom.

"... important [function of] a moderator is to maintain the atmosphere in the audience. It was very interesting! Sometimes Dr. ... would make jokes and lighten up the participants, and that's important because some people almost falling asleep ..." (Respondent 31)

videoconferences involving several sites where people talk with different accents made it harder for all participants and especially presenters because they might not get the question right or simply misunderstand. The moderator's role in such cases was to reduce the tension by paraphrasing the question or comment and obtain verification or correction from the person who asked or commented. By doing so, the moderator can minimise misunderstanding and avoid awkwardness of situations when the presenter starts answering the question without having fully understood the question, as implied in the quote below: 
"She collects [questions], summarises them and puts across to the presenter the summarised questions. That's because people talk with different accents, it is difficult [for the presenter and other participants] to understand them all...whereas she [the moderator] was an Asian..." (Respondent 27)

By her remark above, the respondent 27 made an assumption that an Asian person is more likely to understand the accents of other Asians speaking English than the presenters who are Americans or Europeans.

The moderator's role is complex enough, suggested Respondent 27, so it would be adequate if someone else performed the functions of a facilitator. She noted that either role will be performed better if separated and carried out by different people.

"The moderation and the facilitation both are good when separated. It's too complicated for one person to perform [both functions]." (Respondent 27)

The site moderator can significantly improve the effectiveness of videoconferences if he or she will proactively engage with the local participants prior to the event to clarify their expectations from the session, thought Respondent 29. She also believed that during the videoconference the moderator should ask questions on behalf of the audience because some local participants would rather delegate the question asking task to the moderator than speak themselves, in English and in front of cameras.

"[The moderator should] talk to the Mongolians first and find out their information needs and ... ask questions on their behalf, etc. - probably that could make a difference. If they [moderators] would do that in the future, the relevance, the effectiveness, the impact [of a videoconference] could improve." (Respondent 29)

Ideally, the moderators should not only have good understanding of the subject but also know the audience, especially during the series of videoconferences, believed Respondent 30.

"First of all, [the moderator] should know the subject; they also need to know the people who are invited. They should even anticipate the questions 
that might be asked... for example, "the question you had last time, you can now get an answer from Mr. ..., I've already notified him", and so on, that's moderation already." (Respondent 30)

The above quote implies that the Moderator should chair the actual session as well as communicate with the presenters and organisers between the sessions in order to enhance the effectiveness of communication.

To sum up, the respondents in the Mongolian case reported that the facilitator and moderator roles are equally important and would contribute to more effective communication if performed by two different people. The most important aspects of a facilitator's role, as perceived by the respondents, included prior preparation, careful selection of participants, proactive bridging between the content providers and participants before and during the videoconference and contribution to localisation of the content. The research data suggested that an ideal moderator should possess the following important qualities: time-keeping and floor-management skills, especially during the Q\&A sessions, ability to effectively wrap-up and summarise the main messages, familiarity with the content and participants, and have a sense of humour.

\section{Presentation issues}

Technology apprehension was one of the themes that emerged from the research data, especially when the interviewees discussed the aspects related to presentation. While observing other people's partaking in Q\&A sessions, Respondent 32 felt that some participants avoided detailed conversations when given a floor and their faces zoomed in and shown on the screen.

"For example, a participant asked a question but the presenter didn't understand it and tried to clarify by saying "I thought your question was..., is that right?" Participant then quickly responded "Yes, yes" but it looked like he thought "I'll better just say "yes' because I don't want all people's attention being focused on me", he just wanted that to be over as soon as possible." (Respondent 32) 
Another participant felt that the presenters had a challenge to understand the questions that participants were asking, perhaps due to a difficulty of comprehending different accents of English and poorly formulated question or simply because of bad quality of audio.

"As of the questions asked, I noticed that the two presenters did not quite understand the questions, including those asked from Mongolia. They [participants] were starting by saying 'we have a question' then talking a lot, even we didn't understand what they were trying to ask. Then the presenter would just guess what the question was and say something like "I heard a word 'scope', so I assume your question as about the 'scope'." (Respondent 31)

Respondents 30 noted that a prior distribution of course materials helped the participants to organise their thoughts and prepare the questions in advance, and consequently, facilitated a more efficient interaction of the presenter with the audiences at connected sites.

"Normally, the presenters send the materials in advance and then the World Bank staff forward it to the participants. When we come to the videoconference, already photocopied package of materials lie on the table. So while listening, people just circle those parts where whey would like to ask questions and during the Q\&A sessions ask them and receive answers. So it doesn't affect the effectiveness much." (Respondent 30)

In addition, distributing the presentation materials to the participants before the event is beneficial to both parties because the handouts provide information on the overall scope and level of the topic, and convey the main messages, thus ease the presenter's task of communicating to the multicultural audiences. At the same time, the participants know what to expect from the event and act adequately.

"Because the presentation materials are printed and distributed in advance, people have clear idea and say "oh, I see, the introduction will be held at this level" so they have a certain understanding and have no other expectations. I think that makes it [event] nice." (Respondent 26) 
The presenter can avoid unnecessary questions from the floor by talking to the point, in a coherent manner so that the listeners fully understand the content, thought one of the interviewees:

“The presenter should speak briefly and precisely, with logical sequence, otherwise the listeners will loose the logic, the flow and might miss something. It means, more stupid questions will be asked." (Respondent 26)

Most of the participants believed that the presenters are the experts on their subject topics, thus they can confidently answer the questions raised from the connected audiences, using practical examples. In addition, the following respondent felt that even the participants must be competent enough to answer some questions, especially those related to the local circumstances:

"As far as I feel like... they know the subject, you know? I think, they invite the best, right? Right. So somehow [they should be able to] adequately answer any question, based on 2-3 practical case studies, also the people at the Mongolian site. Normally, the participants know their circumstances really well, so [as the session goes] they just discuss the issues among themselves." (Respondent 30)

Clear articulation of the words, adequate volume of speech and usage of understandable yet sufficient vocabulary is extremely important for the effectiveness of the presentation, believed the following respondent:

"Besides preparing the content, he/she should sound clearly, do not mumble so people are stressed out to hear or shout loudly and distract people's attention. It's important that [the presenter] speaks loud enough so everyone can hear, his words must be clear, his language correct and words precise. Also, the eye contact should be there, although far away, the people should not be looking down and reading from the paper while presenting, right?" (Respondent 26)

The quality and attractiveness of Power Point Presentation, especially the scope of information it contains is highly important for the effectiveness of the presenter, thought one of the respondents. She believed that the slides should 
enclose as much information as possible and be displayed on the screen, not the image of the presenter.

"[Sometimes] the presentation slides displayed separately are different from the presenter's talk, you know? So I think that the presentation is obviously the only way, for now, to display through GDLN. Most importantly, every detail, every specific element should not be neglected. Because why are you bringing all the people at 7-8 sites? There is a presenter, of course. But the presentation slides must be prepared in a really attractive, colourful way, and the presenter should be displayed in a small box on the screen." (Respondent 33)

To summarise, the respondents in the Mongolian case perceived the role of a presenter to be an extremely important one that has an impact on the effectiveness of video-mediated communication. They agreed that for the most effective communication, the presenters must be competent on their subject topics, should talk in understandable terms, with logical sequence and at a sufficient volume, using a set of well-prepared, informative and eye-catching slides. In addition, they believed that prior distribution of the photocopied presentation materials will facilitate a more productive communication and streamline the flow of the Q\&A sessions. 


\section{Chapter 5: Discussion}

The preceding chapter contained interpretation and preliminary analysis of the research data obtained from interviews and observations of videoconference events. The purpose of this chapter is to present the reader with the main themes and sub-themes that emerged from the data across four cases in relation to the research questions of this study. In the search for the answers to the primary research question "What cross-cultural communication factors influence the effectiveness of videoconferencing on development issues as perceived by the participants?" I asked the research interviewees to share their experiences from participating in GDLN videoconferences. From their responses, I learned about their perceptions for the effectiveness of the nature and content of cross-cultural communication that takes place via GDLN and relevance of topics of videoconferences to the development priorities of participating countries. These findings are reported on in Section 5.1, along with the analysis of participants' behaviour during the videoconferences and the role of technology in video-mediated cross-cultural communication as observed by the researcher and complimented by interview data. The first subquestion of the study was "How do the cultural differences influence the effectiveness of videoconferences in perceptions of participants?" This is answered by the explanations in section 5.2. Other factors such as language differences, interpretation and organisational issues that influence crosscultural communication via multipoint videoconferencing are discussed in the last two sections of this chapter. These address the primary research question as well as answer the second sub-question of the current research.

\section{1. Cross-cultural video-mediated communication on development}

This section discusses the nature and content of GDLN videoconferences where people from different cultures communicate through videoconferencing technology. The two most common types of communication in the GDLN context - knowledge transfer and knowledge sharing - are discussed in the following section. Analysis regarding the importance of content of GDLN 
videoconferences and its relevance to the development priorities of participating counties are presented in the section 5.1.2. The last two sections explore the nature of social interaction that takes place during videoconferences analysing participants' behaviour, attitude, communication style and discusses the role that technology plays in this interaction.

\section{1. 1. Knowledge Transfer and Knowledge Sharing}

In their much cited work, Nonaka and Takeuchi (1995) distinguish between tacit and explicit knowledge. Tacit knowledge is experience gained through action, and explicit knowledge refers to knowledge stored and made available in books, databanks or other types of media. Communication on development topics that takes place during GDLN videoconferences involves transfer of tacit as well as explicit knowledge such as well-documented best development practices and covers a wide array of topics ranging from infrastructure financing to anti-corruption initiatives, from fisheries management to publicprivate partnership in infrastructure, from avian influenza to e-government, and many more. Based on observations, I classified GDLN videoconferences into two types: training sessions on particular topics that involve delivery of information and knowledge - in other words, transfer of explicit knowledge; and interactive discussions on broad policy issues that generate mutual dialogue among participants from all connected sites or in other words, tacit knowledge sharing sessions. The first type of communication can be described as largely one-way transfer of particular content from one site to several others simultaneously. The interactive and constructive discussions involving individuals at all participating sites can be described as a two-way or better, multi-way interactive cross-cultural communication. Research participants reflected on their experiences on participation in both types of events.

A document from the World Bank (1999, p. 13) argues that effective communication must be a two-way street because sharing knowledge with the developing world requires an understanding of their needs and concerns and offering knowledge that they can use and will accept. Research data throughout 
four cases suggested that the interviewees attended more videoconferences of knowledge sharing type than knowledge transfer events.

In the World Bank vocabulary, 'knowledge-sharing forums' are Bankfacilitated, client-managed meetings aimed at sharing international "best practice," exchanging knowledge, catalyzing reform, and building consensus on a particular issue (World Bank, 2008). More than half of respondents in the Australia and New Zealand cases reflected on their participation in various sessions of Pacific Leaders' Virtual Forum (PLVF) series of videoconferences on Fisheries Management and Anti-corruption Strategies. Research data from interviews and observation notes suggested that videoconferences of PLVF succeeded in their purpose of generating interactive discussion among culturally diverse audiences because participants at all connected countries were keen to take advantage of rare opportunity to talk to people from other countries via technology. However, participants reported mixed perceptions regarding the effectiveness of videoconferences, especially the four sessions of the Fisheries Management series. Some respondents in the Australian and New Zealand cases, felt that particular topics were too advanced or irrelevant to the realities of participating Pacific nations. At the same time, other interviewees in Australia and New Zealand described these videoconferences as a great opportunity for developing countries to learn from knowing-how or embodied knowledge of their peers in the developed part of the world.

Participants of videoconferences on Anti-corruption policies series considered that the sessions were a great example of knowledge sharing exercise during which participants learnt something new from one another. Topics of knowledge sharing forums differed from country to country but most of them were mentioned as effective communication because during these videoconferences participants were able to discuss issues that are important and relevant to all participating countries. For example, the interviewees in the Russian case referred to their participation in discussions on e-Government, Bologna process and tourism management. The respondents in the Mongolian case reported on a one-off dialogue on Cash benefits scheme held between Ulaanbaatar and Washington that discussed pros and cons of political promises 
prior to the elections, and videoconferences of "Avian influenza" series that linked affected Asian countries and enabled government officials to align policies towards the epidemic.

Throughout the four cases, the research participants agreed that if not enabled by videoconferencing technology such dialogues would most probably not have happened in face-to-face mode due to financial and time constraints. The interviewees suggested that especially for the participants in developing countries, the videoconferences were a rare chance to hear from experts and their peers in other countries as well as share their own experiences. Sometimes, there are security concerns related to travel to developing countries thus videoconference becomes an alternative through which communication can take place. For example, most people would hesitate to travel to Kabul to give a lecture or deliver training courses given the unstable political situation in Afghanistan or go to Timor Leste because of long distance. However, they would happily attend a videoconference that links the Dili DLC, among others, to make a presentation and answer the questions from participants.

"I think it's great that we're able to help or, we seem to be helping engaging Timor Leste in conversation about corruption, and when you see them talking and so interested, you sort of think "wow,... we're actually trying to make some sort of difference"... being as small as it is, I think it gives Timor Leste a voice that they may not necessarily have." (Respondent 1)

The interviewees perceived most of the explicit knowledge transfer type of videoconferences as effective communication. Research participants in the Mongolia case reported on their participation in training courses on Microfinance, ISO quality standards, lecture by Nobel Prize winning economist Dr. Stiglitz, the launch of the World Bank flagship publication and others. Russian interviewees mentioned seminars on business intelligence, tutor preparation courses, etc. The videoconferences were delivered not only from cities in the developed world such as Tokyo, Washington DC or Canberra but also from Chulalongkorn University in Thailand as in the case of Dengue fever program. The data confirmed that the receiving sites were distance learning 
centres in developing countries in the EAP region and Russian Federation. Again, the respondents mentioned that the videoconferencing was an excellent channel for delivery of such courses and seminars because they were cheaper and quicker to organise compared to face-to-face training events. For example, one of the interviewees in the Mongolian case referred to a videoconference that was organised on request of Mongolian government just weeks before the 2004 elections. As a training coordinator of the Mongolian DLC at the time, I witnessed the unprecedented occasion when the President, the Prime Minister and the Speaker of Parliament sat shoulder-to-shoulder with the leaders of opposition in the videoconferencing room in Ulaanbaatar to hear from international experts on other countries' experiences of implementing universal cash transfer programs. Although the experts proposed that making cash promises as part of election campaign was not always effective, the political parties of Mongolia proceeded with announcing various social assistance schemes such as "Child Money" program.

"The "cash transfer videoconference" was the hottest topic at the time... The videoconference gave a very good explanation why cash transfers are ineffective. However, the politicians have already made their decision, they can't go back. So, although the information [provided during the videoconference] was very important, there was no [political] will to accept it. Then of course, the effectiveness [of that videoconference] is poor." (Respondent 29)

The evidence above suggests that videoconferences of an explicit knowledge transfer nature can generate effective communication, but it might not produce the expected outcomes. In this particular case, Mongolian politicians did not want to lose face backing away from promises that have already been made to their respective constituencies. Traditional face maintenance behaviour of Mongolians can be explained by Hall's (1976) cultural dimension of context.

To summarise, the research findings suggest that video sessions of both types knowledge sharing and knowledge transfer - can successfully facilitate mutually beneficial cross-cultural communication on various topics of development agenda. Respondents across all four cases indicated that cross- 
cultural interaction that took place during GDLN events would not otherwise have happened, due to high financial, human resource and time investment required for organising international conferences or training events. Videoconferencing can become an alternative to face-to-face communication with people from countries travel to which is restricted for security or distance reasons.

Comparison of video-mediated communication across cultures revealed similarities in perceptions of participants that videoconferencing technology can be used for information and knowledge transfer as well as knowledge and experience sharing activities to effectively engage audiences across distances, time zones and cultural boundaries.

\section{1. 2. Content of communication and its relevance}

As well as having benefits, globalization of knowledge has the possibility of a darker side because the adoption of an idea without appropriate adaptations might have adverse effect on economic growth, especially when outsiders attempt to impose knowledge on a country without adequate sensitivity to local conditions and social norms (Stiglitz, 2004). The World Bank's approach to knowledge and knowledge transfer assumed that knowledge is universally applicable and it must originate in the 'North' (McFarlane, 2006). For the GDLN, the topics for the courses offered were selected on the basis of their perceived importance from the standpoint of the World Bank staff (AssiéLumumba, 2008).

Research participants in all four cases emphasized that a wide variety in the level of expertise among the participants was hard for all sides engaged in communication. Experts felt frustrated because they had to explain the basic concepts first in order to introduce the main agenda. Participants with lower level of understanding felt embarrassed because they didn't quite follow the communication. The series of videoconferences on fisheries management in the Pacific region was an apparent example of such a situation when Australian professors attempted to introduce complex economic concepts applied in

fisheries management to the participants from Fiji, Papua New Guinea and 
Timor Leste - countries that practice traditional, small-scale fishing. Although these countries share the waters and all belong to the same region, for some participants the concepts under the discussion were too advanced whereas for the session conveners the level of knowledge of targeted audience was inadequate. Here is how Respondent 19 explained the situation:

"The Australians who were running it were putting a very heavily economic, natural resource overlay over it [whereas]... in PNG, Timor Leste, where you have very small, family traditional fishing, [such] economic overlay simply doesn't work, it doesn't make any sense.... On the other hand, there are shared problems: in tuna fisheries around all those nations ... stocks are being depleted so... each of those countries and a region as whole has an interest in addressing that problem." (Respondent 19)

On the other hand, the topic had some relevance to the participating countries each country on its own and all of them as a region had vested interest in finding ways to solve the problem. The fact that the participants from Pacific island nations were eager to learn about the economic concepts of advanced fisheries management was evident from the way they engaged and asked questions as recoded in the observation notes. Therefore, if would be a mistake to conclude that this forum was inappropriately convened or was a complete waste of everybody's time.

One of the presenters of videoconferences on Anti-corruption policies of PLVF series explained how the program design changed as the program evolved over time. According to him, in its first year, the videoconferences were more theory-oriented. Influence of occupational and organisational culture might have been the reason, since the content developers were faculty members of the Australian National University. However, based on participants' feedback, the structure of the series has been modified in the following year and shifted the focus from theory to practice.

"The first one was just introductory, about what were the different meanings of corruption and what people thought about it and everyone 
said that they wanted to do practical, implementation-focused... So I thought we ought to try and focus the discussion a bit more on particular sectors and what you should actually do." (Respondent 6)

The above example demonstrates how content developers used participants' feedback to modify program content and design in order to increase the relevance of videoconferences to the development priorities of participating countries.

The content doesn't have to be directly relevant to development challenges of a particular country's, thought another interviewee and believed that some discussions on cross-cutting issues help countries to look into the problem from a regional or global perspective and provide an opportunity to compare own situation with other countries.

“World Bank knowledge sharing events are not only limited to Mongolia, it gives a new angle from where [we] can look at where Mongolia stands in the region and in the world." (Respondent 33)

As observation notes suggest, the discussions during the second series of videoconferences entitled "Anti-corruption strategies: practical implementation" focused on practical actions related to corruption in particular sectors such as education, land relations and natural resources management. As the interview data and observation notes suggest, the discussions were dynamic, open and constructive. One of the Indonesian participants, who took part in videoconferences from Canberra, expressed his view as follows:

"Having input from participants of other countries on how they are dealing with the corruption ... [was] a new knowledge for Indonesian people and especially for Acehnese people... that will be a part of knowledge that we are bringing home... adopting in our countries later; that will be part of further discussion with our government. Everyone in Aceh is really happy to talk about corruption because corruption has made us living in poverty." (Respondent 3)

As the issue of corruption it is not only a problem of developing countries (Robinson, 1998), so the discussions held during these knowledge-sharing 
videoconferences were relevant to all participants, including those attending from Australia and New Zealand.

In this sub-section, I have discussed the issue of content relevance in GDLN context. The evidence consistently demonstrated that the effectiveness of development communication via video channel increases when videoconference topics relate to the development priorities of targeted audiences. These analyses are produced based solely on the interpretation of data obtained from interviews and observation notes. Although it does not directly apply to the primary research question, I highlighted this as an important finding that should be taken into consideration by people who engage in cross-cultural videoconferences.

\section{1. 3. Nature of social interaction during videoconferences}

The interview data across the four cases suggested that the participants of GDLN events come with goodwill to acquire new knowledge on development issues through discussions, said the interviewees in all four countries. Although coming from different cultural backgrounds, they enter the videoconference room with positive attitude and willingness to learn new information and share own knowledge and experience with their peers in other countries. Oftentimes, excitement of experiencing new technology and being a part of interactive discussions with several countries at once contributed to participants' enthusiasm.

I liken the idea of goodwill to the concept of "swift trust" described by Meyerson and colleagues (1996) how members of temporary groups may be able to accomplish tasks without having developed relationships first. Normally, videoconferences last for just two hours and there is no or little opportunity to allow relationships to develop between the participants. Therefore, I argue that the idea of 'swift trust' is applicable to cross-cultural videoconferencing because participants form a temporary virtual group that works together to accomplish a task of discussion on a particular topic in just two hours. Trust in this case is based on participants' expectations regarding the integrity, competence, skills and knowledge of other participants and 
especially of the presenters because usually the individuals of reputable standing in the field are invited to make presentations during videoconferences. As members of some virtual teams "have never worked together and do not expect to work together again" (Markus, 1994, p. 168), the majority of participants of videoconferences have never met before and do not expect to meet again. However, evidence in this study showed that a basic level of goodwill displayed by the videoconference participants in most cases enables instant yet effective interaction across cultures and languages.

Research data from interviews and observation notes revealed that many participants would not say much during videoconferences. Respondents thought this was due to one or more of the following reasons: lack of knowledge on the subject matter, language barrier, and respect for more authoritative figure or personality traits such as fear of public speaking. Difference of power distances (Hofstede, 1980) affects the conduct of a videoconference as a number of interviewees reported that they hesitated to express their opinions during videoconferences because their superiors were present in the same room. The discussions on power relations during videoconferences will be presented in the next section.

Evidence from this study suggested that different cultures can effectively discuss a sensitive topic such as corruption, over videoconferencing. Using one's position for personal advantage is simply a common sense in many, if not most, countries (Fisher, 1997) and ideas of reciprocity and gift-giving as 'part of culture' have often been used to explain, or excuse, acts of corruption (Larmour, 2008). Therefore, discussing the issues of corruption must have been a difficult task for some of the participants. However, as research data suggested, they were openly sharing the corruption practices in their respective countries. As one of the respondents noted:

"We had Timor Leste come in and they were discussing corruption with the rest of the region. And that to them was culturally not acceptable to really discuss this, so they won't talk about it but they have problems with it. But then they saw the way the other counties are handling it ... now they are only too happy to be involved." (Respondent 2) 
As can be seen from the study data, the videoconferencing channel can effectively facilitate cross-cultural communication over a tense or sensitive topic such as bribery and corruption. Interviewees proposed that most likely, participants would have not opened up as much if they were discussing an issue of corruption with their local counterparts and in a face-to-face setting.

\section{1. 4. Impact of Technology on Cross-cultural Communication}

The role of the video-conferencing technology on the participants' perceptions for the effectiveness of cross-cultural mediated communication emerged as another finding of this study. The impact of technology has two forms: one is more straightforward and concerns various technical problems related to the videoconferencing system or internet connection, whereas the other form is more complex and concerns the way by which this technology influences the way people behave and communicate during cross-cultural videoconferences.

The first form of impact can be resolved as information and communication technologies progress and find wider practical application in distance education, organisational communication, telemedicine and other sectors. An interpretive case study on the use of videoconferencing technology for elearning in Bolivia highlights the technology as one of the challenges in facilitating cross-cultural communication across distances and time zones (Stödberg \& Orre, 2010). Underdeveloped IT infrastructure is a common problem in the developing word where most of the GDLN affiliates are located. Interview data and observation of videoconferences provided ample evidence of technology failures ranging from minor disruptions such as freezing picture of the far end to complete cut off of one of the sites from the session.

The more significant finding of this study concerns the second form of implications of videoconferencing technology on general process of crosscultural communication. Research data suggests that this technology brings people together because they are able to interact with each other in real time seeing the faces and body language of the participants on the other side and hear their voices, different intonations and accents and laugh at the jokes they make. 
As perceived by research participants, the technology affected participants differently: in some cases, it enhanced and in other, it impeded the effectiveness of cross-cultural communication.

Some interviewees - especially those who participated in a videoconference for the first time - felt self-conscious to see themselves on the screen, talk to the microphone and be heard at all remote audiences simultaneously. This is partly related to different cultural norms, for instance, in some countries it is culturally inappropriate to be very frank and vocal because it is considered to be rude and therefore, generally, people are more inclined to be polite and selfeffacing. However, they admitted that the anxiety diminished soon after the session began.

Others felt empowered by the technology and used the rare opportunity to observe, and interact with their peers from different parts of the world. A number of interviewees reported that the technology enabled them to act in a way they normally would not choose to or have a chance in a face-to-face setting. For example, they were able to reduce power distance by communicating with top level officials and asking questions on pressing issues directly (the Russian case), overcome personal traits like shyness and practice their English without feeling self-conscious (the Mongolian case) or discuss sensitive issue like bribery despite to varying level of uncertainty avoidance among the participants (the Australian case). The majority of interviewees recalled that they felt excited to be part of virtual interaction and appreciated the occasion.

The all-familiar concept of 'time is money' is directly relevant to videoconferencing due to connectivity costs that vary from site to site. Therefore, video sessions are highly structured, have precise running order and predefined floor-taking arrangements. Thus, quick and to-the-point responses in discussions with little introductory phrasing or politeness is highly valued in videoconferencing environment, although the direct plan approach of linear time conception can be viewed as rude by some participants that come from polychronic cultures (Limaye \& Victor, 1991). 
The above findings suggest that the videoconferencing technology has an influence on how people behave and communicate when engage in crosscultural events. Moreover, the technology affects the way they perceive the effectiveness of cross-cultural video-mediated communication.

\section{2. Impact of Cultural Differences of participants on communication}

From the data, certain key concepts emerge that describe how participants perceive the effects of culture on video-mediated cross-cultural communication, These include differences of participants in terms of mindsets, cultural diversity and difference of educational systems in four countries. The various levels of culture - national, occupational and organisational - that influence communication are examined in sub-section 5. 2. 2.

\section{2. 1. Cultural diversity of participants: opportunity or obstacle?}

\section{Difference of mindsets}

Difference of mindsets emerged as a recurring theme from the research data because a number of respondents throughout four cases referred to words 'mentality', 'mindset', 'way of thinking and doing things', and 'the way we/they are'. The most efficient way to understand international behavioural differences is to look for significant differences in mindsets imposed by the culture and in the standard operating procedures which define the way individuals in the group are expected to perceive and reason about the world around them (Fisher, 1997, p. 45). Respondents in the Russian and Mongolian cases addressed issues of 'mindset' and/or 'mentality' with respect to themselves more often than interviewees in the Australian and New Zealand cases. They talked about 'socialist legacy' and 'communist regime' that had shaped the frame of mind of people to a large extent.

"We had no choice. Everybody had to line in rows... It was very hard for people to shift from a system in which everyone is obliged to everyone and all were lining in rows. Now, you have to make a choice [for] yourself..." (Respondent 11) 
Informants in the Russian case referred to the 'Russian' or 'Eastern' mentality as opposed to 'Western' mindset, whereas interviewees in the Mongolian case talked about Mongolians in relation to 'other Asians' and 'Westerners'. Moreover, interviewees recognised cultural diversity within one nation state. For example, Respondent 29 distinguished the mindset of a 'hybrid' Mongolian whose worldview is being shaped by a complex blend of multiple cultural layers in accordance with the virtual onion model (Gallivan \& Srite, 2005) e.g. Russian and/or American, on top of their 'Mongolian core'. On the other hand, some respondents in the Russian case referred to the mindsets of the 'new Russians' and 'post-Soviets' as opposed to 'Soviet people' in general and population of 'former Soviet republics' in particular. However, from the fastpaced transition of Russia came a generation of younger Russian managers who now exhibit more entrepreneurial inclinations, welcome democratic leadership style, value individual achievement, and do not like to discuss family and personal problems at work (Matveev \& Milter, 2004). Half of the interviewees in the Russian case were under the age of thirty and fit this description. Interview data and notes taken on viewing recordings of videoconferences involving Russian DLC suggest that the majority of Russian participants government officials, NGO representatives, businessmen, researchers - were enthusiastic, outspoken and confident young people.

Studies suggest that since the collapse of the USSR there has been a steady increase in cultural differences between Russians and nationals of former Soviet republics; and these are now considerably greater now than in the Soviet period (Kolosov, Galkina, \& Krindach, 2003).

While Russian is the predominant culture with the Russians accounting for about 80 percent of the total population or nearly 116 million people, over 150 other national groups coexist in the Russian Federation (Zorin, 2004). In comparison, Mongolia's cultural background is mostly homogenous with more than 85 percent of population or 2,2 million people being of ethnic Mongol descent and the rest belonging to a handful of other ethnic groups (General Authority for State Registration of Mongolia, 2007). 
A recent cross-cultural study (Sablonnière, Tougas, \& Lortie-Lussier, 2009) examined consequences of social change in Russia and Mongolia after the dismantlement of the Soviet Union. The authors concluded that the effects of social restructuring in Mongolia were more positive than in Russia, as Mongolia gained status worldwide with the creation of a democratic political system promoting new cultural values whereas in contrast, Russia has lost its worldwide superpower status with the fall of communism. Perceptibly, the dramatic changes that took place in two countries must have had different yet considerable effects on the mindsets of its people.

In comparison, people in Australia and New Zealand - the other two countries of this four country comparative study - have been enjoying a rather stable political, economic and social life for the last few decades. It is reasonable to assume that the mindset of the Australians and New Zealanders did not undergo such dramatic changes as the worldview of the people in Mongolia and Russia.

The increasing diversity of population in Australia and New Zealand provides a rich mix of cultures, languages, religions, traditions and activities (Australian Bureau of Statistics, 2006; Statistics New Zealand, 2007). Almost 44 percent of Australians were either born overseas or had at least one overseas-born parent (Australian Bureau of Statistics, 2006) whereas 22.9 percent of people usually living in New Zealand were born overseas (Statistics New Zealand, 2007). A number of respondents in the Australian and New Zealand cases regarded the communication that took place during videoconferences as any other communication in their everyday life. They teach to international students or work in a culturally diverse workplace; and friends or members of extended family originate from different cultures. During the videoconferences most of them spoke English, their first language. On the other hand, their confidence in the effectiveness of communication could have been an explanation to disguise their ignoring the cultural differences of people they communicate with.

\section{Cultural diversity}


A number of respondents in the Russian case talked about cultural diversity in Russia. Active migration inflows and outflows stemming from the disintegration of the USSR resulted in radical changes in the ethnic composition of Russian population (Zorin, 2004). When discussing cultural differences and talking about "foreigners", they used a term "near and far abroad" ("ближнее и дальнее зарубежье" in Russian) that became a common idiom after the collapse of Soviet Union. The "near abroad" meant territories of former Soviet republics whereas the "far abroad" implied "the rest of the world". Thus, the Russian respondents viewed cultural differences from two perspectives: Russian culture as opposed to the culture of other nationalities populating Russian Federation and countries of former Soviet Union; and Russian culture compared with other cultures around the world.

At the same time, Mongolian respondents viewed the communication during videoconferences as being cross-cultural indeed. Firstly, they had to listen to and speak a foreign language or via interpretation. Secondly, cultural diversity in the workplace, academic environment or at family level is a rare and unusual occurrence in the largely mono-cultural Mongolian society.

Interviewees reported that cultural differences of participants were visible in the ways people looked, talked and acted. Respondents observed that participants from different cultures display certain characteristics when participating in videoconferences: Australians talk fast, have an accent that is difficult to understand, like to gesticulate; most Mongolians are reserved, selfconscious and formal but some women can be more talkative and assertive than men; Nepalese are frivolous, like to laugh and poke jokes at others; people from the Philippines speak English well; Cambodians, Vietnamese, and Chinese are well-organised and duly follow the course requirements; Indians, Bangladeshi and Pakistani people have good English skills but have heavy accents, talk a lot and fast and like engaging in debates; and so on.

\section{Difference in educational systems}

The research data suggests that difference in educational systems, including that within one country, plays a significant role in the way how participants 
communicate. For example, Respondent 29 mentioned that the Mongolians who went to Russian secondary schools are different from their peers who went to Mongolian schools. While explaining their own or other people's behaviour, the respondents in the Russian and Mongolian cases referred to 'the schooling we went through', opposed "the Russian educational system' to "the Western educational system" and so on. The difference of educational systems is important because in some countries, educators put emphasis on analytical thinking, creativity and competitiveness whereas in others, practice of memorising and repeating is a norm and student achievements are compared to fixed standards (Fisher, 1997). As a result, people think, operate and perceive things differently because the values and norms around them have been set differently - as the research participants attested. Therefore, the difference in educational standards in participating countries may impact the effectiveness of communication during videoconferences.

The majority of interviewees throughout the four cases viewed the cultural diversity of participants positively and generally agreed that the cultural differences of participants enriched the communication, made it more interesting. According to social identity theory (Tajfel \& Turner, 1985), people generally strive to achieve a positive identity by making favourable comparisons between their own group and other relevant groups (Brown, 2000). A respondent in the Mongolian case commented that different cultural backgrounds of participants 'triggered comparative thinking and creativity' (Respondent 31).

At the same time, some interviewees in the Australian and New Zealand cases suggested that videoconferences take place in a culture-free virtual zone where the differences are 'masked off' or significantly reduced. They felt that communication over videoconferencing is 'acultural': issues that are sensitive in a given country can be less sensitive when discussed with other countries. "It's an odd kind of transnational space that everybody is operating in", noted Respondent 6.

These observations illustrate that the impact of cultural differences on communication is perceived differently by different cultures. On one hand, 
Australian and New Zealand participants think that acultural virtual environment of videoconferences reduced the communication barriers that difference of cultures could normally put up in a face-to-face mode. On the other hand, Mongolian and Russian participants viewed cultural diversity positively and thought that the communication held was more interesting and differences generated relative thinking.

Some research participants referred to a certain 'videoconferencing culture' in which communication is formal, highly structured and time-limited. A number of informants suggested that national cultural differences do not interfere in communication if the participants work on the same project. For example, interviewees in the New Zealand and Mongolian cases (Respondents 24 and 25) reflected on their participation in working meetings that discussed progress of a development project. The interviewees referred to different sets of meetings in which they participated separately at different times. Both witnessed how people of different nationalities and from different organisations effectively communicated over videoconferences. The impact of occupational and organisational culture on the effectiveness of communication is discussed in the next section.

Interpretation of data in this cross-cultural comparative study revealed that cultural dimension of time (Hall, 1976) is highly relevant to intercultural communication that takes place during videoconferences. According to this model, time is not perceived as linear in many cultures (polychronic) where people frequently view time as flexible and communicate in indirect or circular manner. Limited time disempowered participants from Pacific countries, commented Respondent 5, 'because it takes a little bit of time ... [for them] to relax and open up..., you can't just go in and have this business conversation ... you need to have a conversation rhythm, and start off slowly'. She suggested that participants from cultures where people talk straight to the point with little introductory phrasing (monochronic) should adapt their communication style to some extent when participating in videoconferences with people from different cultures. Research participants across all four cases noted that highly structured, time-limited nature of videoconferences was a challenge for 
effective cross-cultural communication because they differ from the perspective of the cultural dimension of time and have different expectations forwards one another. However, most of them proposed that facilitators, moderators and presenters can play a critical role in reducing the time pressure and enabling productive interaction. Further discussion on organisational issues including facilitation, moderation and presentation is presented in Section 6.4.

In interpreting and analysing the research data, I employed the concepts of low context and high context cultures (Hall, 1976). For example, a couple of interviewees illustrated how some videoconference participants made wrongful assumptions based on observed behaviour of people involved in videoconferences. Some Russian participants felt that by inviting a particular African country to join the same videoconference with Russia, the organisers (World Bank) underestimated Russia's expertise. An innocent joke made by a western presenter was perceived by Russian participants as offensive. Two Mongolian interviewees felt that frequent bursts of laughter at one site (Nepal) were a sign of disrespect towards the participants at other sites (India, Sri Lanka, Philippines). Another example demonstrated how just one phrase can have a rich contextual meaning for some cultures but mean nothing for others: a speaker from Israel used an expression 'nine-eleven' during a videoconference that linked several Russian cities. Fortunately, the translator knew that in America and Europe, these two numbers refer to the notorious terrorist attack that took place in America on the 11 th of September in 2001. Although the message in this particular occasion was conveyed correctly, the chances of misinterpretation were high. These anecdotes demonstrate the importance of the environment in which the communication takes place and contribute to validation of Hall's (1976) context model that has been criticised by Cardon (2008) for a lack of empirical evidence. .

This section has provided an account of analysis of respondents' views regarding the difference of mindsets, cultural diversity of the videoconference audiences and differences in educational systems in their respective countries. Based on the findings, I have deduced the answer to the question posed in the heading of this sub-section: "Cultural diversity of participants: opportunity or 
obstacle?" The answer is: the cultural diversity of the participating groups presented more opportunities than obstacles. Different cultural backgrounds of the participants enriched the communication, made it more interesting and dynamic. The participants perceived the effectiveness of communication from different mindset perspectives because the historical, political, economic and social background in their respective countries differed drastically.

\section{2. 2. Impact of occupational and organisational culture}

This cross-cultural study compared the communication phenomena in four national cultures and two sub-cultures: occupational and organisational. People of different nationalities that work on similar jobs, or in other words belong to the same occupational group, have common values and worldview (Ashforth \& Mael, 1989; Hofstede, Neuijen, Ohayv, \& Sanders, 1990). Research data comprised discussions about various occupational groups: development practitioners, people engaged in micro-financing, policy-makers, university lecturers, government officials, NGO representatives, etc. Respondents throughout the four cased reported that the occupational culture had more influence on the nature of communication than national culture. For instance, a respondent in the Australia case talked about the "split between different streams: academics and practitioners" (Respondent 6) across five national cultural groups that participated in the same videoconference. The other Australian interviewee thought that the culture did not matter as much as the "severe differences among the groups in terms of their stakes in the fishery" (Respondent 4)

Some respondents who believed that national cultural differences had no influence on the effectiveness of communication mentioned common occupational grounds to support their view. One of the Russian interviewees thought that cultural differences in terms of languages, habits, traditions had little influence on communication about e-services in public sector because all governments perform similar functions (Respondent 12).

Another Russian interviewee implied that generally, the organisers of videoconferencing events were more interested in participants from the 
perspective of occupational rather than national culture simply because only the professionals of high level of expertise were able keep their peers in other countries motivated and engaged in fruitful discussions (Respondent 14)

Like national cultures, occupational sub-cultures, too, develop distinct languages or jargon (Hansen, 1995). A Mongolian respondent echoed this idea by saying:

"People who work in this industry generally speak the same language banking or micro-finance language - and for that you don't need to have [knowledge of] literary English." (Respondent 27)

In parallel with discussions on occupational culture, the data points towards the influence of some strong organisational cultures e.g. the World Bank, Asian Productivity Organisation, on communication. For instance, a representative of the Mongolian National Productivity Centre believed that people working for similar organisations in other countries were "like one family, specialise in particular topics so almost speak the same language" (Respondent 28)

As an Australian respondent believed, the World Bank has a "very strong technocratic culture - despite the fact that their staffs represents a wide variety of cultures - generally everybody working there and contractors with experience all share the same basic set of concepts and constructs" (Respondent 24).

All of the above suggested that occupation and organisational cultures also have significance for the nature of communication, in addition to the impact of national culture. This finding contributes to the field of research on occupational cultures that according to Hofstede and colleagues (Hofstede, Neuijen, Ohayv, \& Sanders, 1990), have received considerably less attention in the literature than either national or organizational cultures.

\section{2. 3. Knowledge and experience gap}

Participants of videoconferences differ in terms of knowledge and experience on the topic of communication. Discussion in this section relates to and 
elaborates on the earlier text about knowledge sharing and knowledge transfer types of GDLN video sessions presented in Section 6.1.1.

What divided the developed world from the less developed countries was not only a gap in resources, but also a gap in knowledge (Stiglitz, 2004). This view is supported by research participants because respondents in all four cases acknowledged that such a gap had more impact on the effectiveness of communication than just differences of national cultures. Some respondents argued that the communication effectiveness was reduced when knowledge levels of participants differed significantly. For example, a New Zealand interviewee (Respondent 19) felt that a mismatch of degrees of knowledge and experience hindered the communication during a videoconference on fisheries management. A Russian interviewee (Respondent 10) noted that a different level of economic development among participating countries and consequently, a different level of knowledge of participants on specific topics had more influence on the effectiveness of cross-cultural communication than national cultural differences. He mentioned that such a gap in knowledge is also applicable to communication that takes place between CIS countries.

The data suggests that the lack of knowledge can be on the either side of communicating parties: content providers and content recipients. Respondents suggested that sometimes, the expertise of presenters on the content providing site does not necessarily meet the expectations of audiences in content receiving countries. For example, during a videoconference that linked Washington with several DLCs in the Russian Federation, one of the participants openly criticised the American presenter for the lack of local knowledge. Respondent 14 who observed this incident, reported as follows:

“They [participants] are demanding from the experts - and it's fair enough - a better knowledge of the situation in the region for being able to give advice. I think the experts should listen to them because it's a feedback from those whom you're giving recommendations." (Respondent 14) 
The above quote suggested that videoconferences provided knowledge sharing opportunities for all parties engaged in communication: experts presented their ideas and the participants from the developing countries provided feedback and shared their local knowledge on the subject matter. One of respondents in the Australian case also emphasised the importance of local knowledge in the effectiveness of communication:

"When you deal with participants, like ... from Timor, it is very important for the presenters to understand the [local] situation and be able to engage the participants." (Respondent 7)

However, it was not always easy to spot the disparity in levels of understanding on the topic. An interviewee in the Australian case realised the extent of knowledge gap only when participants started asking questions during Q\&A sessions:

"The questions they were asking were not the questions [indicating] that they misheard; these were questions [implying] that they didn't understand because the level of knowledge was so far behind [than] the other people's." (Respondent 5).

Examples of videoconference on topics such as e-government (Russian case) and fisheries management (Australian and New Zealand cases) suggested that knowledge levels may differ significantly between the participating countries at the content receiving end. For instance, some Russian officials refused to participate in a videoconference on off-shore programming because they felt that Russia is way more advanced in this sector than their colleagues from an African county that were invited to join this videoconference so that they do not have much common grounds to hold a joint discussion. A number of informants stressed that communication between the professionals working in different countries with similar occupation and level of expertise make crosscultural communication more focused and productive.

Research data suggested that the participants had diverse, sometimes opposite, experiences in relation to the subject of discussion. For instance, a New Zealand respondent noted: 
"The other difficulty ... is that the experience of the various nations was extremely different. So, you have Australia and New Zealand well versed in modern fisheries management and you had a number of other [Pacific] nations with traditional fisheries. ... So it was in some sense cultural but in some sense simply a knowledge and experience gap." (Respondent 19)

An interviewee in the Mongolian case also mentioned how drastically different were the practices of food production and safety, for example, in a nomadic herding household in the Mongolian steppes versus a fully automated dairy farm in New Zealand. Data revealed that the diversity of practices was beneficial for the communication: "people understand these differences and think 'how can we implement it in our country, what opportunities do we have?" (Respondent 31, Mongolia); "what they probably did get is an appreciation that the problems they're facing are being faced by others" (Respondent 19, New Zealand); "their experiences may vary and be equally relevant and interesting to the other parties" (Respondent 10, Russia).

Several participants thought that cultural differences can successfully be bridged and effectiveness of communication increased if communicating groups of people have a similar level of expertise on the topic. Respondents especially those who were involved in the selection of videoconference participants - noted that professional qualities and level of expertise were more significant for communication outcome than national culture of the participant. Observation data suggested that the 'mixing' of varying experiences created new, enhanced competence in the process of communication and mutual learning. Such discussions enriched the content, expanded the knowledge on the topic and 'triggered creativity and comparative judgment' as commented one of Mongolian interviewees (Respondent 28).

Research participants noted that videoconferences in GDLN context were not simply 'transfer of North-originated knowledge to the third world' (McFarlane, 2006) but a productive multi-way exchange of knowledge and experience among geographically dispersed peers and colleagues. Based on the above discussion, it is possible to contemplate that the effectiveness of interactive 
knowledge sharing activities such as cross-cultural videoconferences will increase if communicating groups will have similar level of knowledge on the topic of discussion. However, the differing experiences of participants based on their respective cultures, traditions and way of living enriched and broadened the communication.

\section{2. 4. Power issues}

Power distance refers to the degree of inequality among people that the society expects and accepts; conceptions of power vary from culture to culture and may be based on rank, skill, knowledge, and connections (Hofstede, 2001). Research participants throughout the four cases provided examples of power relations in cross-cultural videoconferencing, at various levels.

In some situations, the way participants communicated and/or conducted themselves suggested that they were feeling a pressure of superior-subordinate relationship. For example, one of the Australian interviewees reported on one such incidence:

"Once when the Fiji lot was settling down and suddenly the minister6 appeared ... so, everybody was rapidly tailoring their opinions to what they were going to say..." (Respondent 6)

A Russian interviewee brought an example when "there was a bit of a tense atmosphere because participants in Moscow were bosses whereas at the other sites were their subordinates". However, he thought that videoconferencing technology reduced the power distance because communicating parties were geographically dispersed.

"It was more open than a conversation that would've taken place if these officials were called to come to the ministry office in Moscow... All sorts of questions were asked, even some questions that would've not been asked if the communication was face-to-face." (Respondent 13)

\footnotetext{
${ }^{6}$ At that time, Fiji's military commander Frank Bainimarama has been controlling the country after the December 2006 coup that brought international condemnation and economic sanctions from donors (BBC News, 2006). The Fijian 'Minister' who participated in the VC was a member of interim government appointed by Bainimarama. In April 2009, the Fiji's Court of Appeal have found that the 2006 military coup was unconstitutional and have ordered that democracy be restored by holding fresh elections (McClymont, 2009).
} 
A New Zealand interviewee believed that there is "often a distinction between what people feel obliged to say and what they actually think", for example when one thinks "I don't want to say too much because I'm not really comfortable with the people I'm in the room with" (Respondent 23).

In some cases, research participants reported on relationships between the dominant culture/s and other participating countries. One of the respondents explained the relationship between Australia and New Zealand as "a friendly rivalry, certainly, there's also a sense in which it's a big country and we, in New Zealand at least, don't like being told what to do" (Respondent 19), whereas a Mongolian interviewee was confident that neither of the communicating parties acted like "I'm from a superior nation, you're from a poor country" (Respondent 30). The Russian case contained examples of 'cultural arrogance' when Russian participants felt that they were much more sophisticated than their African colleagues because historically, the former USSR used to provide development assistance to some nations in Africa. A respondent in the Australian case mentioned that "in Timor, accessing people is easier, while in Australia it's a bit hard to go and talk to them, you have to make appointments" (Respondent 7).

The research data contains evidence of relations between donor organisation such as World Bank and countries that are recipients of official aid. A Mongolian respondent was confident that during some videoconferences "the power difference was obvious [because] the World Bank experts have a tendency to guide, direct and tell what they have to tell, top-down" (Respondent 29). One of Russian interviewees mentioned that the "World Bank opinion is very influential" and government officials tend to think that criticism from their side is "an unfavourable international PR" (Respondent 10) whereas a New Zealand interviewee suggested "the key stakeholder groups in New Zealand... have a variety of opinions about the role of the World Bank and the development community ... didn't want to become significantly involved in the World Bank strategies, they wanted to be involved in regionally alliance strategies that actually work on meeting identified needs" (Respondent 21). 
Donor legitimacy concerning the provision of policy advice is declining due to various perceptions ranging from apparent economic and financial system failure in the developed world to the individual inexperience of donor officials. Such perceptions affect the attitudes of recipient governments revealing a growing scepticism about the technical advice they continue to receive from foreigners and some degree of bemusement and irritation at the manner of its delivery (Blunt, Turner, \& Hertz, 2011).

To summarise, the research data contained evidence of various power relations: superior-subordinate, large versus small countries, more developed versus less developed nations, donor versus recipient countries.

\section{3. Language Differences and Interpretation}

The culturally and linguistically diverse participants of GDLN videoconferences face multiple challenges when communicating. This section will discuss how lingual diversity of participants influenced the communication. The role of simultaneous interpreters in bridging the language barrier will also be discussed.

\section{3. 1. Language differences}

People from diverse national cultures with different national languages and value systems join together in GDLN videoconferences. In two of the four countries under the study - Australia and New Zealand - English is the national language and mother tongue to the majority of population. In 2006, English was spoken by 95.9 percent of New Zealanders (Statistics New Zealand, 2007) while 83 percent of Australian population (Australian Bureau of Statistics, 2006) spoke only English at home. Whereas the other two countries - Russian Federation, where the official language is Russian and Mongolia, where Mongolian is the national language - have the same problem: just a very small percentage of videoconference participants speak or understand English. Therefore, most of the GDLN videoconferences discussed in this research took place through simultaneous interpretation. 
When interacting over cultural boundaries in English, people are often under the false impression that they are sharing the same context and interpretation, that is that the same expressions and words have the same connotations for everyone (Peltokorpi \& Clausen, 2011).

The greater part of research informants believed that language difference is a barrier that can easily be solved. However, several interviewees across the four cases argued that the message does not get fully transmitted and something does get 'lost in translation' when translation is involved, more so when communication is simultaneously interpreted.

"Often, even with the most capable of the interpreters ...you very rarely completely, fully understand the specific meaning of what the other person is trying to say." (Respondent 9, Australia)

"In synchronous translation, only the general meaning is transmitted." (Respondent 34, Russia)

"The problem with interpreters is you're never quite sure whether they're interpreting you correctly. And the more technical a discussion, the less sure you are." (Respondent 24, New Zealand)

"There is no way that a 100 percent translation can be achieved, no matter how good the translator is..." (Respondent 26, Mongolia)

Perhaps, at a superficial level, language barrier can be overcome by simultaneous translation, but it makes deeper concepts more difficult to convey from one language to another instantaneously.

\section{3. 2. Translator/Interpreter as cultural mediator}

One of the major ethical requirements for interpreters states that an interpreter is not allowed to give his/her opinion, or to alter in any way what a speaker expresses through his/her language: "The formulation of the message is the responsibility of the other parties; the interpreter's responsibility is to interpret" (Gentile, Ozolins, \& Vasilakakos, 1996, p. 48). However, Taft (1981, p. 59) states that "[ ...] mediation between cultures requires the communication of ideas and information from one cultural context to the other. This is analogous to the 
process involved in linguistic translation, even though there is more to mediation than mere translation".

As the main language of GDLN videoconferences is English, the respondents in the Australian and New Zealand cases did not require services of an on-site interpreter. Most of the other interviewees had experience of participating in videoconferences that were simultaneously interpreted by translators located at the remote sites. Therefore, this research contains interviews with three interpreters only: two from Mongolia and one from Russia.

Many interviewees unanimously agreed that simultaneous interpretation of videoconferences is a highly complex and demanding task. Given that literal translation is generally not considered advisable except for fairly technical terms (Visson, 1999), it is at the interpreter's discretion to decide the extent to which he/she should 'mediate' rather than merely 'translate' to improve the communication flow. Two interviewed translators used a similar approach when interpreting video-mediated communication - they avoid literal translation and focus on conveying the main idea concisely:

"I try to understand the main concept of what they are talking about. If the essence is clear, the details can be added later... Otherwise, sentenceby-sentence translation is impossible." (Respondent 29, Mongolia)

"In synchronous translation, only the general meaning is transmitted... Frankly speaking, only the subject, predicate etc: Who? Did what? When?" (Respondent 34, Russia)

A couple of respondents suggested that the feelings are not fully transmitted when simultaneous interpretation is employed. They believed that "the translators usually smooth out a little bit, don't convey all the emotions" (Respondent 14) and choose words that are more appropriate in receiving language. One of the translators admitted that she skips "some emotional utterances" of speakers (Respondent 34, Russia). On the contrary, the other translator made every effort to convey the passion and expressiveness of the speakers and believed that monotonous speech "switches off the human brain" (Respondent 29, Mongolia). 
Technical aspects such as audio transmission delay, even as short as a quarter of a second, made it harder for the interpreters because awkward situations created by pauses frequently result in overlapping speech, e.g. when a participant starts to restate his or her question just as a delayed reply from the remote site arrives.

Informants in all four cases agreed in general that the effectiveness of translation-mediated communication will increase relative to the degree of the interpreter's knowledge on the subject matter. A few people also acknowledged that it was hard to find skilled interpreters, especially those who would be well versed on the topic of discussion. The danger was noted of situations when an interpreter without specialist knowledge on the topic of discussion might mislead the participants by erroneous or incomplete translation of specific terms or complex concepts. The interviewees felt that ideally, the interpreter should be well aware of cross-cultural communication issues and mitigate any misunderstanding and confusion.

Based on the above, I conclude that in some cases, the role of a simultaneous interpreter of GDLN videoconferences goes beyond mere translation and includes a fine art of a cultural mediator, thus significantly influences the effectiveness of cross-cultural communication.

\section{4. Organisational factors}

In the search for the answers to the primary research question, I invited the respondents to discuss factors that influence the effectiveness of GDLN events. In addition to the technology (Section 5.1.4), cultural differences of participants (Section 5.2) and issues related to language barriers (Section 5.3), research informants across four cases talked about several other aspects of the videoconference process that contributed - positively or negatively - to the effectiveness of GDLN events. They agreed that the factors such as facilitation, moderation and presentation play a significant role for the effectiveness of cross-cultural videoconferences. By presenting the findings regarding these aspects in the following sub-section under the umbrella heading 'Organisational issues', I argue that each of them is a cross-cultural 
communication factor that influences the effectiveness of GDLN events. This section therefore contributes to answering the primary research question of this study: "What cross-cultural communication factors influence the effectiveness of videoconferencing on development issues as perceived by the participants?"

\section{4. 1. Facilitation and Moderation}

The distinction between the functions of a facilitator and moderator is discussed in section 1.1. Most respondents throughout the four cases used terms 'facilitator' and 'moderator' interchangeably because they thought that the two roles are quite similar. However, some research participants, especially those whose work was closely related to GDLN, had a very distinct understanding on each of the roles and argued that the two functions would be best performed by different people. Data illustrated how overwhelming it can be to juggle many tasks simultaneously when the same person acts as a moderator and facilitator simultaneously.

The literature (Berge \& Collins, 1995; Knipe \& Lee, 2002), including the practitioner-based literature (Marziali, Donahue, \& Crossin, 2005; Van Ast, 2005) suggested that site facilitators play a significant role in shaping the participants' perception regarding the effectiveness of videoconferencing events. According to research data, responsibilities of a videoconference facilitator included all or some of the following tasks: selection of and communication with the potential participants and speakers; dissemination of background materials; communication with and coordination of actions between moderators, presenters, translators and participants. The interviewees reported that in most cases, the facilitators welcomed the participants into the videoconference room and provided a short briefing about the set up, demonstrated the use microphones and headsets; offered technical support in case of minor flaws and liaised with the facilitators at other connected sites and network support staff at the hub. A few respondents mentioned that they also need to have a back-up plan in situations when things do not go as expected; gather feedback from the participants at the end of the session/s either by a questionnaire or through informal communication. For example, in the 
Australian case, facilitators wrote proposals to potential donors seeking funds for developing new videoconference events and actively participated in the design and implementation of these programs. At the same time, the observation notes confirmed that facilitator arranged for tea/coffee and light refreshments for the participants and cleaned the videoconference room afterwards. In Russia, facilitator handled the financial aspects of videoconference organisation and performed a function, highly political at times, of finding the most optimal balance in composing the communicating groups. Occasionally he had to step in to help if the translator straggled to cope with the complexity or pace of the videoconference.

The facilitator's ability to control the running of videoconferencing equipment and technical trouble-shooting skills allowed the communication to proceed smoothly and avoid disruptions and surprises, recalled the interviewees. Ideally, a facilitator should instruct the presenters and interpreters on the specifics of video-mediated cross-cultural communication compared to face-toface interaction, commented some interviewees.

More frequently mentioned by research participants were interpersonal skills ability to engage with participants and encourage fruitful discussion - as the most important quality that a facilitator - and a moderator - should possess. Words like "dynamic, interactive, enthusiastic, persuasive, resourceful" were used by the interviewees when they discussed other attributes of a successful facilitators as well as moderators.

The Moderator plays a vital role in the effectiveness of videoconferences: he/she can make or break a successful event, agreed the research participants. By effectively chairing the discussion and keeping everybody motivated and engaged the moderator can significantly improve participants' perception of the effectiveness of communication. The majority of respondents throughout the four cases believed that good communication and time-management skills are the key qualities which the videoconference moderators should possess. Following the running order or altering it as necessary to make the best use of the allocated time is not an easy task. However, managing the floor is easier in videoconference environment than in face-to-face setting because only one 
microphone can be active at one given time, concluded some respondents. Data suggested that in some cases, the moderator airs the questions on behalf of the participating audiences to the panel of speakers or makes instantaneous prioritising from the pool of questions and comments. Given the multilingual audience of the videoconference, the moderator should also be able to understand different accents of English - that of participants and simultaneous interpreters at connected sites - in order to effectively summarise and paraphrase the question or a comment in a way that is understandable to the presenter. In the Russian case, the moderator had to fill in the gap and converse with the remote audiences when the audio from the content delivering DLC has been off for a few minutes.

The moderator, or a chairperson, needed to closely follow the discussion and wrap up the session or its parts by summarising the key aspects. The interviewees believed that people with outstanding interpersonal skills and a great sense of tact and time can enable effective communication by competently moderating the sessions. One respondent reflected on some operational videoconferences that he attended in the past - some were moderated and some were not - and concluded that those with a moderator that followed a pre-set agenda had been more efficient than unstructured group discussions.

Although the two roles seemed quite similar, some respondents identified clear differences. For example, while moderators acted in the live, real time communication, given a floor and shown on the screen, the facilitators' predominantly worked off the screen and their efforts often were left unnoticed and taken for granted if not major interruptions were involved.

\section{4. 2. Presentation}

Presenters' experience in cross-cultural communication played a significant role for the effectiveness of communication. Research data suggested that university lecturers and people who belong to a culturally diverse workplace display higher intercultural competence. A couple of interviewees in the New Zealand case believed that generally, the New Zealanders were more culturally aware compared to Australians (Respondents 19 and 21). However, given greater 
cultural diversity in Australia, it is unlikely and the research data cannot confirm this statement.

Making a presentation in front of a camera, pinned to one spot and without eye contact with the listeners is not the same as lecturing before the live audience, walking back and forth and gesticulating, agreed most of the interviewees, especially university lecturers.

Research data suggested that participants' perception of the effectiveness of cross-cultural communication can be reduced if the camera is wrongly placed. As it has been noted by several respondents, lack of eye contact between the presenter and participants is one of the downsides of videoconferencing. On the other hand, cultures perceive eye contact differently: some find it rude or confronting, whereas other understand it as a sign of openness and closeness. However, technicians can help in reducing the effects of this disadvantage by placing the camera at such an angle that captures the speaker's front view, suggested some interviewees. This will give the participants an impression that they are being looked at by the presenter "so that the other sites would perceive the dialogues as live, more close to the real [face-to-face] situation" (Respondent 10).

Some research participants did not like presenters who used many slides, talked monotonously for too long or extensively used technical terms and corporate jargon. However, they appreciated when presenters used plain English and articulated the words clearly. As discussed in the section 9.3, different accents were difficult to comprehend, especially during one-off videoconferences when participants did not have sufficient time to get accustomed to different pronunciations.

Technology apprehension of presenters and other speakers was another theme that emerged from data. For example, a Mongolian respondent noticed that some participants avoided making long comments when given the floor because they felt embarrassed by seeing own image on the big screen and audiences at all remote sites attentively listening to what he/she has to say. 


\section{5. Chapter summary}

The most important findings of this research were reported on in this Chapter. Analysis of the social interaction that takes place during cross-cultural videoconferences revealed that knowledge transfer as well as knowledge sharing takes place during GDLN events. Knowledge and knowledgeable people made the differences of cultures less evident. The big gap between the knowledge and experience levels was viewed by some as a barrier to effective communication whereas others looked at it positively and noted that different realities of participating countries enriched the interaction. The technology certainly influenced people's behaviour and communication during videoconferences as well as their perceptions for the effectiveness of crosscultural communication.

This chapter also elaborated on how cultural differences of participants national, organisational and occupational - influence the communication and concluded that cultural diversity is viewed as an opportunity rather than an obstacle in cross-cultural videoconferences. Issues of power relations, language barriers, interpretation, facilitation, moderation and presentation were also discussed as factors that influence - some to more and some to a lesser extent the effectiveness of cross-cultural videoconferences, as perceived by research participants. 


\section{Chapter 6. Conclusions and Implications}

This dissertation has reported on a cross-cultural comparative study of the many factors that affect video-mediated communication across cultures. More specifically, this research examined how cultural differences of participants among other factors identified - influence the effectiveness of cross-cultural communication during multi-point room-based videoconferences on development issues.

The research method used in this study was multiple-case study of explorative nature which guided the collection and analysis of the research data that comes from semi-structured interviews conducted in four countries in three languages, notes taken during observation of videoconferences and other relevant documents. This four-case study research has taken a comparative approach in analysing the data in light of theoretical models informing it such as the cultural dimensions as elaborated by Hall (1976) and later by Hofstede (2001) and the Virtual Onion metaphor as illustrated by Straub (2002) based on Social Identity Theory (Tajfel \& Turner, 1985).

In this final chapter I present the main research conclusions of the study and then examine the implications for future research and practical recommendations for development practitioners.

\section{1. Research Conclusions}

The context of this study is the Global Development Learning Network of the World Bank that facilitates dialogue on development issues using videoconferencing technology. Much of what is written on the strategy of development is produced by people whose point of view is from the outside looking in, that is, from the perspective of specialists from the developed world who diagnose and prescribe for a client country people whose real-life experience they have not shared, as noted by Fisher (1997). Contrary to that major trend, this research was conducted by, and reported on, by a practitioner who facilitated communication on development with over 15 years of experience of working from the inside, in a developing country. 
The data was analyzed to answer the primary research question "What crosscultural communication factors influence the effectiveness of videoconferencing on development issues as perceived by the participants?" and the two secondary questions:

- How do the cultural differences influence the effectiveness of videoconferences in perceptions of participants?

- How can the factors identified be used to bridge these cultural differences for more effective communication?

Understanding the effectiveness of media is necessary to the understanding of effective organizational communication (Fulk \& Boyd, 1991) but the effect of cultural differences on the perception of media effectiveness has not been delineated in past research, despite numerous studies across cultures to try to determine the best means of communicating (Leonard, VanScotter, \& Pakdil, 2009). The research that is reported in this thesis makes an original contribution to the scholarship relating to video-mediated communication using empirical data collected in different cultural settings and applying established theoretical models by Hall (1976), Hofstede (1980) and Tajfel \& Turner (1985).

In summary, the answer to the primary research question is as follows: the effectiveness of cross-cultural communication on development issues that takes place through videoconferencing technology is influenced by the following factors: 1) diversity of national and organisational cultures; 2) disparity in the levels of knowledge and experience of participants; 3) the technology; 4) language differences; and 5) organisational aspects such as facilitation and moderation. The following sections will present more detailed analysis of these factors and present answers to the secondary research questions using the findings from this qualitative inquiry.

\section{1. 1. Cultural diversity and videoconferencing}

This comparative analysis of perceptions of research informants for the effectiveness of cross-cultural communication suggests that cultural differences of participants of videoconferences - on the levels of national and 
organisational culture - found similarities as well as differences across the four countries.

Observation notes and interview transcripts suggested that there were significant cultural differences at the national level: in ways participants behaved and communicated among themselves and with people from other countries, used the time, perceived humour, etc. as presented in descriptions of four country cases in Chapter 4 and in discussion analysis in Chapter 5. For example, the communication style of Germans, Americans and Australians (low context, polychronic cultures) was structured and specific whereas Mongolians, Timorese and Vietnamese (high context, monochromic cultures) used long introductory phrases before asking a question or expressing an opinion, often quite indirectly. Differences in economic development and lifestyles were apparent when participants used examples from their respective countries in relation to various concepts. For example, Japanese discussed issues of quality and productivity in the case of Toyota production system whereas Mongolians talked about quality and safety of meat and dairy produced in the nomadic household. Research data contained evidence of how people from different cultures perceived humour differently, and how they displayed different approaches to learning process - largely due to the differences of mindsets that have been shaped over time by traditional cultural norms and values, educational systems, political regime and other influences. Russians, Australians and New Zealanders live in societies where there is more exposure to different cultures thus they perceived communication that took place during videoconferences just as normal communication whereas Mongolians (largely monocultural society) recognised this interaction as a rare opportunity of engaging in a communication with the foreigners. However, this research finding needs further exploration.

Research data contained evidence of power distances that existed between developed economies and developing nations (Japan and Mongolia) as well as between the large and small countries in the developed part (Australia and New Zealand) and developing part of the world (Indonesia and Timor Leste). 
Diversity of organisational cultures among the international participants also displayed commonalities and disparities. For example, people from different countries that belonged to the same umbrella organisation (Asian Productivity Organisation) shared common goals, understood each other better because used the 'same corporate language' (concepts and terminology) and operated under common principles. Despite a large variety of organisational and occupational representation, all participants generally demonstrated motivation to interact with colleagues, learn new information relevant to their work and share own experiences and knowledge as well as establish useful contacts and relationships with their peers from other countries.

Government officials, civil society, private sector and donor community representatives displayed different communication patterns when interacting during videoconferences because they adhered to dissimilar organisational cultures depending on their professional affiliation. As research data confirmed, power distance between government representatives of different ranks (minister and other public servants in PNG) was higher than between superiors and subordinates of international donor organisation (e. g. World Bank).

Table 5 Cultural differences and similarities at national and organisational levels

\begin{tabular}{|c|c|c|}
\hline & National culture level & Organisational culture level \\
\hline 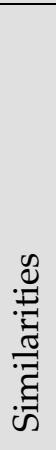 & $\begin{array}{ll}- & \text { Good will, positive attitude } \\
\text { - } & \text { Motivated to experience new } \\
& \text { technology } \\
- & \text { Interested to interact with } \\
& \text { people from other cultures } \\
-\quad & \text { Willing to learn new } \\
\text { information and share own } \\
\text { knowledge and experiences }\end{array}$ & $\begin{array}{l}\text { - Willing to learn new information } \\
\text { and share own knowledge and } \\
\text { experiences } \\
\text { - } \quad \text { View as an opportunity to establish } \\
\text { working relationships with peers } \\
\text { from other countries } \\
\text { - } \quad \text { Understand the nature of work and } \\
\text { common professional terminology } \\
\text { - Affiliates of the same umbrella } \\
\text { organisation share common goals }\end{array}$ \\
\hline 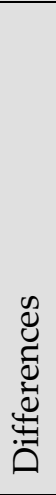 & $\begin{array}{ll}- & \begin{array}{l}\text { Perception of time } \\
\text { (polychronic/ monochromic) }\end{array} \\
- & \text { Power distance (high/low) } \\
- & \text { Power relations } \\
& \text { (developed/developing } \\
& \text { economy; large/small country) } \\
- & \text { Multicultural/Monocultural } \\
& \text { society } \\
- & \text { Communication patterns } \\
- & \text { Mentalities; Perception of } \\
& \text { humour }\end{array}$ & $\begin{array}{ll}\text { - } & \text { Power relations } \\
\text { (superior/subordinate) } \\
\text { - } & \text { Communication patterns depending } \\
& \text { on affiliations (government/NGO/ } \\
\text { development partner/private } \\
\text { sector) }\end{array}$ \\
\hline
\end{tabular}


Table 5 (above) distils the earlier analysis and summarises the differences and similarities at the level of national and organisational culture.

Based on the data analysed, I argue that people from different national, organisational and occupation cultures can effectively employ videoconferencing technology for constructive communication on development issues. For example, a follow-up meeting on an infrastructure project may involve World Bank consultants (international development institution), specialists from a contracted firm (business company), and officials representing the implementing agency (government organisation), environmental activists (NGO) and researchers (academia). Their nationalities and occupational affiliations may vary: for example, American project management specialist, German engineer, Mongolian accountant, social activist, graduate student, and so on.

Overall, research participants perceived the cultural diversity - at national and organisational levels - positively and agreed that cultural differences of participants enriched the communication and facilitated a richer communication. Data confirmed that the technology reduced the communication barriers that cultural differences could normally put up in a face-to-face mode by bringing all into a virtual, a-cultural space.

\section{1. 2. Knowledge and experience gap}

Respondents in all four countries agreed that the gap in the level of knowledge and experience among the participants had greater impact on the effectiveness of communication during videoconferences rather than national or organisational cultural differences. This lack of knowledge was on either side of the communicating parties: content providers and content receivers. Research participants reported on cases when presenters had inadequate knowledge about the current realities of participating countries (an American presenter was criticised by Russian participants for being under-informed on current situation in Russia) or vice versa: the participants had no basic knowledge about the topic or the level of discussions was too advanced (course on fisheries management was too technical for Timor Leste participants). 
The problem of knowledge and experience gap could possibly be solved by careful selection of participants and proper preparatory work on the content provider side. The study reported on several cases of highly efficient crosscultural interactions conducted over a video connection when invited participants were professionals in the same field or of similar level of knowledge $\mathrm{n}$ the topic of discussion. Evidence suggests that cross-cultural teams working on the same or similar projects had the most efficient operational videoconferences. A carefully designed mix of participants' knowledge and experiences created new and enhanced existing competences and enabled productive and effective communication.

The research data triangulated by different sources across all four cases, especially in the Russian case, suggest that important relationship exists between knowledge and culture. I therefore argue that knowledge and knowledgeable people diminish cultural differences of audiences participating in the same videoconferencing event and increase the effectiveness of such interaction.

\section{1. 3. Role of technology and Videoconferencing culture}

The synchronous nature of this technology enables instantaneous feedback, allows various groups of people to engage in communication that otherwise would have not taken place.

The research data suggested that cross-cultural interactions that take place during GDLN events introduce a certain 'videoconferencing culture' that participants have to immigrate into in order to effectively communicate with people from diverse cultural backgrounds.

Formal, highly structured and time-restricted nature of videoconferences sets aside the national, organisational and occupational cultural differences. Some participants from polychronic cultures may feel disempowered by time limitation of videoconferences because they need a while to open up, so often they start up slowly by saying pleasantries and long introductions. On the contrary, participants from monochronic cultures are better placed to make the 
best use of the time allocated for the videoconference. The most efficient communication will be made possible if participants from different cultures adhere to a set of common rules.

The research data confirms that the videoconferencing environment reduced power distance and enabled discussions over sensitive issues such as corruption and equal interaction between superiors and subordinates that were at different locations. Videoconferencing technology also helped to build relationships, because participants - members of a temporary group established swift trust (Meyerson, Weick, \& Kramer, 1996) that facilitated instant yet effective communication across different cultures and language barriers. Respondents also reported that in the videoconferencing setting the participants experienced less concerns regarding deficiencies in their English language skills.

Thus, the videoconferencing technology, or in other words, a 'technology factor', influences - more positively than negatively - the participants' perceptions for the effectiveness of mediated cross-cultural communication.

\section{1. 4. Role of the simultaneous interpreter}

The language difference among the participants was an important factor that influenced the effectiveness of cross-cultural videoconferences. Hence, the outcome of video-mediated interaction was highly dependent on the knowledge and professional skills of the simultaneous interpreter. Where the conference was multilingual, evidence triangulated by interviews and observations suggests that the quality of translation had a significant influence on the perceptions of participants for the effectiveness of videoconferences. The translators faced many challenges: different pace of speech (native speakers versus people for who English is the second language); different accents of English, use of various professional terms and technical/organisational jargon, etc. Participants generally believed that the language barrier can be solved with the help of a good simultaneous interpreter, although they unanimously agreed 
that simultaneous interpretation of videoconferences is a highly complex and demanding task.

The interviewed translators admitted that it is almost impossible to instantaneously translate difficult concepts in full, so to some extent, portions of the content are 'lost in translation'. Data suggests that a mediocre translation could create a negative impression among the participants about the quality of the content or credibility of a presenter. On the contrary, a superior translation by a person who is well versed on the topic of discussion can enhance the value of the content or increase the perception towards the presentation skills of the lecturer.

Translators often do not only interpret content from one language to another but also act as cultural mediators because cross-cultural communication involves transfer of ideas and information from one cultural context to the other (Taft, 1981). The research informants who translated videoconferences usually focused on conveying ideas and avoided literal translation of the speech and it was usually at their discretion to decide the extent to which they should 'mediate' rather than merely 'translate' to improve the communication flow. However, this cultural mediation had a different nature than that of the facilitators and moderators.

\section{1. 5. Bridging the cultural differences}

The research suggests that the cultural differences summarised in Table 5, effects of technology and knowledge/experience gap can be minimised to some extent by skilful facilitation and moderation of videoconferences. A mismatch in selection of participating countries or participants' level of expertise/knowledge on the topic affected the effectiveness of videoconferences on development topics. In other words, facilitators and moderators can reduce the cultural differences among the participants, both at national and organisational levels.

As discussed in the preceding section, a simultaneous interpreter can also significantly help in bridging the cultural differences and minimising the 
communication breakdowns. Development of a strong videoconferencing culture with a complete set of in-house rules to which participants will be asked to adhere to could also diminish barriers that differences of cultures could otherwise put up.

\section{1. 6. Verification of theoretical models informing this research}

Hall's (1976) model of context is often criticised for being based on dated, unsubstantiated claims and anecdotal evidence and lack of scholarship that empirically validates it (Cardon, 2008; Kittler, Rygl, \& Mackinnon, 2011). The findings of this qualitative cross-cultural enquiry contributed to addressing this criticism by providing new qualitative data collected from interviews conducted and observations made in four cultures - Australia, New Zealand, Mongolia and Russia - that substantiates the dimensions of context and time.

This study suggests that the cultural dimension of power distance (Hofstede, 2001) is relevant to cross-cultural video-mediated communication on development issues. Illustrations of power distance at societal level and examples of its operationalisation at the individual level: differences between the perceptions of participants from large industrialised countries and small developing nations, superiors and subordinates, experienced presenters from international organisations and participants at the content receiving sites with different affiliations were recorded in all four cases.

The data contained in this study contributes to the body of knowledge related to an individual's social identity for cross-cultural communication. Every culture requires a particular pattern of communication, which generally develops over centuries. However, this research validates that individuals within one culture do not necessarily conform to the expected societal patterns when engaged in cross-cultural communication over a videoconference; they display a wide variety of communication styles depending on the blend of different 'layers' of social identity and experiences they possess (Gallivan \& Srite, 2005): personality traits, education, multi-cultural experience, acquaintance with the technology, etc. 


\section{2. Implications for future research}

The findings of this study, synthesised from an extensive pool of data triangulated by different data sources, contribute to the body of knowledge relating to the effectiveness of cross-cultural communication that is conveyed through video channel. It addressed some gaps in the literature that have been listed in the section Avenues of Future Research of Introduction Chapter. For instance, it illustrated the impact of cultural diversity of videoconference participants on the effectiveness of communication in a global videoconferencing network. Also, this research examined the impact of technology on the nature of social interactions that take place during videoconferences.

In responding to the necessity of bridging cultures, the specialists and practitioners in development communication have to develop multicultural capabilities themselves to be able to successfully facilitate the process of interaction across cultures. Further studies could examine:

- Effective use of information and communication technologies including videoconferencing - for knowledge sharing, capacity building and policy advise in development communication.

- Intercultural competence - combination of concepts, attitudes, and skills necessary for effective cross-cultural communication.

- The role of social media technologies as alternative channel of communication or ways in which these media can be used in conjunction with videoconferencing.

- The ways of maximising positive and minimising negative impacts of technology on the effectiveness of cross-cultural videoconferences.

These are critical areas for research for this increasingly globalising world where need for effective communication via technology is important not only in the development domain but also in organisational communication, distance education and other areas of global affairs. 


\subsection{Recommendations for practitioners}

This qualitative study was conducted to inform organisers and participants of video-mediated interactions on development by identifying some of the challenges they might experience in respect of cultural and language differences of participants or organisational matters such as moderation and facilitation of events. This section answers the second sub-question of my research - How can the factors identified be used to bridge cultural differences for more effective communication? - and provides practical recommendations to organisations that use videoconferencing technology for communication across geographical and cultural boundaries. Ideas expressed in this section will be of special interest to actors and agencies working in the field of international development.

- Sharing a common understanding is crucial for the effectiveness of videoconferences. Therefore, all parties engaged in communication would benefit from streamlining the concepts and terms that are relevant to the topic of communication.

- Presenters should avoid presenting generically developed materials. As a preparation for the videoconference, they need to study the situation in participating countries, look at the composition and level of knowledge and experience of participants and tailor the presentations accordingly. Presenters need to pay attention not only to the content of presentation but also to the style in which the material will be presented. Preparing a list of anticipated questions and potential answers would be helpful.

- Presenters should apply culture-specific (e. g. US style) training designs with precaution because the audience may not accept American or European communication, cognitive and learning styles (Bennett \& Bennett, 2001). For example, sending extensive reading materials to the participants of non-training videoconferences assuming that they will come to the session fully prepared e.g. having read the materials, would be a mistake.

- Adequate organisation of a videoconferencing room set-up would contribute to the effectiveness of communication: optimal placement of 
camera angle can create an illusion of eye contact between the presenter and participants at remote locations.

- For the videoconferencing sessions to run smoothly, the technical infrastructure needs to be fully tested to prevent possible problems from occurring during the actual session.

Multilateral and bilateral organisations, international NGOs and multinational corporations that use videoconferencing technology for communication across cultures might increase the effectiveness of interactions by applying the set of practical recommendations described above. 


\section{References:}

Adler, N. J. (2002). International dimensions of organizational behavior (4th ed.): South Western, Thomson Learning.

Al-Qinai, J. (2004). Convergence and Divergence in ranslating vs Interpreting competence. AUMLA: Journal of the Australasian Universities Modern Language Association, Nov(102), 61-84.

Albirini, A. (2006). Cultural perceptions: The missing element in the implementation of ICT in developing countries. International Journal of Education and Development using Information and Communication Technology 2(1).

Allen, M., Bourhis, J., Burrell, N., \& Mabry, E. (2002). Comparing Student Satisfaction With Distance Education to Traditional Classrooms in Higher Education: A Meta-Analysis. American Journal of Distance Education, 16(2).

Allen, M., Sargeant, J., Mann, K., Fleming, M., \& Premi, J. (2003). Videoconferencing for practice-based small-group continuing medical education: Feasibility, acceptability, effectiveness, and cost. Journal of Continuing Education in the Health Professions, 23(1), 38-47.

Alshare, K., Kwun, O., \& Grandon, E. E. (2006). Determinants of Instructors' Intentions to Teach Online Courses: A Cross-Cultural Perspective. The Journal of Computer Information Systems, 46(3).

Andreasen, A. R. (1994). Social Marketing: Its Definition and Domain. Journal of Public Policy \& Marketing, 13(1), 108-114.

Andreev, A. L. (2008). Russians' Mentality and Problems of Social Inequality. Sociological Research, 47(5), 52-62.

Andrews, K. (2005). Teaching and learning through broadband videoconferencing. Unpublished Dissertation, University of Alberta, Canada.

Archer, S. (1988). 'Qualitative' research and the epistemological problems of the management disciplines. In A. Pettigrew (Ed.), Competitiveness and the Management process (pp. pp. 256-302). Oxford: Blackswell.

Arnold, T., Cayley, S., \& Griffith, M. (2002). Videoconferencing in the classroom: communications technology across the curriculum: Davon: Devon Curriculum Services.

Arunachalam, S. (1998). How the Internet is failing the developing world Paper presented at the Ninth International Conference of the International Federation of Science Editors, Egypt.

Ascroft, J. (2006). A conspiracy of courtesy. In A. G. Dagron \& T. Tufte (Eds.), Communication for social change anthology (pp. 71-75): Communication for Social Change Consortium, Inc.

Asgill, A., \& Bellarmine, T. (2003). Effective Distance Delivery of Technical Courses Through Interactive Instruction: Experiences in Delivering Technical Content at a Distance. Paper presented at the Southeastern Section of 2003 American Society for Engineering Education Annual Conference, Nashville, Tennessee, USA.

Ashforth, B. E., \& Mael, F. (1989). Social Identity Theory and the Organization. The Academy of Management Review, 14(1), 20-39.

Assié-Lumumba, N. D. (2008). The Global Development Learning Network (GDLN) and Tertiary Education in Africa: Opportunities and Predicaments of Centralized Knowledge Broadcasting Programs on the World Scale. Perspectives on Global Development and Technology, 7(3-4), 231-258. 
Australian Bureau of Statistics. (2006). Cultural diversity overview [Electronic Version]. A Picture of the Nation: the Statistician's Report on the 2006 Census, 34-44. Retrieved 11 August 2009 from http://www.ausstats.abs.gov.au/ausstats/subscriber.nsf/LookupAttach/2070.0Pub lication29.01.0910/\$File/20700 Cultural overview.pdf.

Badenhorst, Z., \& Axmann, M. (2002). The educational use of videoconferencing in the arts faculty: shedding a new light on puppetry British Journal of Educational Technology, 33(3), pages 291-299.

Bare, J.-F. (1998). Of loans and results: Elements for a chronicle of evaluation at the World Bank. Human Organization, 57(3).

Bekkering, E., \& Shim, J. P. (2006). i2i Trust in Videoconferencing. Communications of the ACM, 49(7), pp. 103-107.

Bell, N. (2007). How native and non-native English speakers adapt to humor in intercultural interaction. Humor: International Journal of Humor Research, 20(1), 27-48.

Benbasat, I., Goldstein, D. K., \& Mead, M. (1987). The Case Research Strategy in Studies of Information Systems. MIS Quarterly, 11(3), pp 369-386.

Bennett, J. M., \& Bennett, M. J. (2001). Developing Intercultural Competence: An Integrative Approach to Global and Domestic Diversity. Paper presented at the Conference Namel. Retrieved Access Datel. from URLI.

Bennett, M. J. (1998). Basic Concepts of Intercultural Communication: Selected Readings: Intercultural Press

Berge, Z., \& Collins, M. (1995). Computer mediated communication and the online classroom Cresskill, N.J. : Hampton Press, c1995.

Berger, P. L., \& Luckmann, T. (1966). The Social Construction of Reality: A Treatise in the Sociology of Knowledge. New York: Doubleday.

Berger, P. L., \& Luckmann, T. (1972). In H. C. Triandis (Ed.), The Analysis of Subjective Culture. New York: John Wiley.

Bernard, R., Abrami, P., Lou, Y., Borokhovski, E., Wade, A., Wozney, L., et al. (2004). How does distance education compare with classroom instruction? A MetaAnalysis of the empirical literature. Review of educational research, 74(3), pp. 379-439.

Blunt, P., Turner, M., \& Hertz, J. (2011). The Meaning of Development Assistance. Public Administration and Development, 31(3), 172-187.

Bonham, M. G., Surin, A. V., Nakano, M., \& Seifert, J. W. (2003, June 23-28). The Transformative Potential of IP Videoconferencing in Graduate Professional Education: Collaborative Learning in Japan, Russia, and the United States. Paper presented at the ED-Media 2003: World Conference on Educational Multimedia, Hypermedia, \& Telecommunications, Honolulu

Bower, B. (2001). Distance education: Facing the faculty challenge Online Journal of Distance Learning Administration, 4(2).

Braa, J. M., Eric; Sahay, Sundeep. (2004). Networks of Action: Sustainable Health Information Systems Across Developing Countries. MIS Quarterly, 28(3).

Braun, S. (2007). Interpreting in small-group bilingual videoconferences: Challenges and adaptation processes. Interpreting, 9(1), 21-46.

Britz, J. J. (2007). A critical analysis of information poverty from a social justice perspective. University of Pretoria, Pretoria.

Brown, K. G., Rietz, T. A., \& Sugrue, B. (2005). The Effects of Videoconferencing, Class Size, and Learner Characteristics on Training Outcomes. Performance Improvement Quarterly, 18(1), 59-82.

Brown, R. (2000). Social identity theory: past achievements, current problems and future challenges. European Journal of Social Psychology, 30(6), pp. 745 - 778. 
Burke, S. C., Chaney, B. H., \& Kirsten, W. (2010). International Videoconferencing for Public Health Education: Linking the U.S. and Germany. American Journal of Health Education, 41(1), 53-59.

Burn, J., \& Thongprasert, N. (2005). A culture-based model for strategic implementation of virtual education delivery. International Journal of Education and Development using Information and Communication Technology, 1(1), 32-52.

Burns, J. T. (2002). Evaluation of Staff Development and Training Models To Support Implementation of Videoconferencing Technology for Teaching and Learning in a Distributed University. Quarterly Review of Distance Education., 3(3), p327340

ByeByeBlighty.com. (2008). Worldwide Cost of Living Survey 2008 (Vol. 2010): Bye Bye Blighty.

Campbell, J. (1996). User perceptions of videoconferenced meetings as a fanction of technology, group size and communication task. Accountability and Performance, 2(2), pp. 15-35.

Campbell, J. (1997). The Impact of Videoconferenced Meetings on the pattern and Structure of Organisational Communication. Singapore Management Review, 19(1).

Campbell, J. (1999). Communication Apprehension and Participation in Videoconferenced Meetings. Paper presented at the 10th Australasian Conference on Information Systems.

Campbell, J. (2006). Media Richness, Communication Apprehension and Participation in Group Videoconferencing. Journal of Information, Information Technology, and Organizations, 1, 87-96.

Cardon, P. W. (2008). A Critique of Hall's Contexting Model: A Meta-Analysis of Literature on Intercultural Business and Technical Communication. Journal of Business and Technical Communication, 22(4), 399-428.

Carlson, P. J., \& Davis, G. B. (1998). An investigation of media selection among directors and managers: From "Self" to "Other" Orientation. MIS Quarterly., 22(3).

Carville, S., \& Mitchell, D. R. (2000). 'It's A Bit Like Star Trek': The Effectiveness of Video Conferencing. Innovations in Education and Teaching International $37(1)$.

Cerulo, K. A. (1997). Reframing Sociological Concepts for a Brave New (Virtual?) World Sociological Inquiry, 67(1), 48-58.

Chaney, L. H., \& Martin, J. S. (2003). Intercultural Business Communication (3d ed.): Prentice Hall

Chen, K. (1999). Internet-based videoconferencing in cross-cultural learning: A case study of an experiment between a Canadian school and a Chinese school. Unpublished Dissertation, Queen's University at Kingston, Canada.

Chidambaram, L., \& Jones, B. (1993). Impact of communication medium and computer support on group perceptions and performance: A comparison of Face-to-Face and Dispersed Meetings. MIS Quarterly, 17(4), p. 465 (427 pages).

Choi, H. J., \& Johnson, S. D. (2005). The Effect of Context-Based Video Instruction on Learning and Motivation in Online Courses. American Journal of Distance Education, 19(4), 215-227.

Chua, W. F. (1986). Radical Developments in Accounting Thought. The Accounting Review, 61(4), pp. 601-632.

Clark, H. H. (1996). Using language. Cambridge, U.K.: Cambridge University Press. 
Clarke, M., Butler, C., Schmidt-Hansen, P., \& Somerville, M. (2004). Quality Assurance for Distance Learning: A Case Study at Brunel University. British Journal of Educational Technology, 35(1), p5-11

Cole, C., Ray, K., \& Zanetis, J. (2004). Videoconferencing for K-12 Classrooms: A Program Development Guide: The International Society for Technology in Education.

Colladas Ais, A. (1998). La evaluacion de la calidad en interpretacion simultanea: La importancia de la comunicacion no verbal. Albolote (Granada): Editorial Comares

Connection., C. C. (1987). Chinese Values and the Search for Culture-Free Dimensions of Culture. Journal of Cross-Cultural Psychology, 18(2), 143-164.

Coventry, L. (1996). Video Conferencing in Higher Education. Edinburgh: Institue for Computer Based Learning, Heriot Watt University.

Daft, R. L., \& Lengel, R. H. (1984). Information Richness: A New Approach to Managerial Behavior and Organizational Design. In L. Cummings \& B. Staw (Eds.), Research in Organizational Behavior (pp. 191-233). Homewood, IL: JAI Press.

Daft, R. L., \& Lengel, R. H. (1986). Organizational Information Requirements, Media Richness and Structural Design. Management Science, 32(5).

Daft, R. L., Lengel, R. H., \& Trevino, L. K. (1987). Message Equivocality, Media Selection, and Manager Performance: Implications for Information Systems. MIS Quarterly, 11(3), pp. 355-366.

Darke, P., Shanks, G., \& Broadbent, M. (1998). Successfully Completing Case Study Research: Combining Rigour, Relevance and Pragmatism. Information Systems Journal. , 8, pp 273-289.

Dennis, A., Valacich, J., Speier, C., \& Morris, M. (1998). Beyond Media Richness: An Empirical Test of Media Synchronicity Theory. Paper presented at the 31st Annual Hawaii International Conference on System Sciences, Hawaii.

Dennis, A. R., \& Garfield, M. J. (2003). The adoption and use of GSS in project teams: toward more participative processes and outcomes. MIS Quarterly, 27(2), p289$323,235 \mathrm{p}$.

Dennis, A. R., \& Kinney, S. T. (1998). Testing Media Richness Theory in the New Media: The Effects of Cues, Feedback, and Task Equivocality. Information Systems Research, 9(3), 256 (219 pages).

DeSanctis, G., Wright, M., \& Jiang, L. (2001). Building a global learning community. Communications of the ACM, 44(12), 80-82.

Doherty, N. F., \& Doig, G. (2003). An analysis of the anticipated cultural impacts of the implementation of data warehouses. IEEE Transactions on Engineering Management, 50(1), pp. 78-88.

Drisko, J. W. (1997). Strengthening qualitative studies and reports: Standards to promote academic integrity. Journal of Social Work Education, 33(1), p. 185 (113 pages).

Dunlop, T. (2003). If you build it they will come: Blogging and the new citizenship [Electronic Version]. Evatt Foundation publications. Retrieved February 17, 2009 from http://evatt.labor.net.au/publications/papers/91.html.

Dustdar, S., \& Hofstede, G. J. (1999). Videoconferencing across cultures - a conceptual framework for floor control issues Journal of Information Technology, 14(2).

Eisenhardt, K. M. (1989). Building Theories From Case Study Research. The Academy of Management Review, 14(4), p. 532 (519 pages)

El-Shinnawy, M., \& Markus, M. L. (1997). The poverty of media richness theory: explaining people's choice of electronic mail vs. voice mail. International Journal of Human Computer Studies, 46(4), 443-467. 
Elenkov, D. S. (1998). Can American management concepts work in Russia? A crosscultural comparative study. California Management Review, 40(4), 133-156.

Escobar, A. (1995). Encountering development: the making and unmaking of the Third World.: Princeton University Press.

Falconer, D., \& Mackay, D. (1999). The key to the mixed method dilemma. Paper presented at the 10th Australasian Conference on Information Systems.

Federman, M. (2006). On the Media Effects of Immigration and Refugee Board Hearings via Videoconference. Journal of Refugee Studies, 19(4), 433.

Ferguson, J. (1994). The anti-politics machine: 'development', depoliticization, and bureaucratic power in Losotho. Minneapolis, MN: University of Minnesota Press

Ferry, D. L., Kydd, C. T., \& Boyles, C. (2012). Creating the Global Graduate: A CrossCultural Videoconferencing Case Study. Decision Sciences The Journal of Innovative Education, 10(2), 139-164.

Fisher, G. (1997). Mindsets : the role of culture and perception in international relations (Second ed.): Intercultural Press.

Ford, D. P., Connelly, C. E., \& Meister, D. B. (2003). Information Systems Research and Hofstede's Culture's Consequences: An Uneasy and Incomplete Partnership. IEEE Transactions on Engineering Management, 50(1), 8-25.

Fulk, J., \& Boyd, B. (1991). Emerging theories of communication in organizations. Journal of Management, 17(2), 407-446.

Fulk, J., \& Dutton, W. (1984). Videoconferencing as an organizational information system: Assessing the role of electronic meetings. . Ssystems, Objectives, Solutions, 4(2), pp. 105-118.

Fulk, J., Schmitz, J., \& Steinfield, C. (1990). A social influence model of technology use. In J. S. Fulk, C. (Ed.), Organisations and Communication Technology (pp. 117-142). Newbury Park, CT: Sage.

Gallivan, M., \& Srite, M. (2005). Information technology and culture: Identifying fragmentary and holistic perspectives of culture. Information and Organization, $15,295-338$.

Gao, Y., Lui, Y., \& Mahanti, P. K. (2006). Implementation of e-learning in the western China: a review. Paper presented at the 17th IASTED international conference on Modelling and simulation Montreal, Canada

Gass, S., \& Varonis, E. (1984). The Effect of Familiarity on the Comprehensibility of Nonnative Speech. Language Learning, 34(1), 65-89.

General Authority for State Registration of Mongolia. (2007). Ethnic groups of Mongolia. Retrieved 12 August, 2009, from http://www.citizenmongolia.com/index.php?option=com_content\&view=article \&id=122:2009-06-19-06-31-47\&catid=53:2009-06-19-06-28-14

Gentile, A., Ozolins, U., \& Vasilakakos, M. (1996). Liaison Interpreting: A Handbook. Melbourne: Melbourne University Press.

Gerster, R., \& Zimmermann, S. (2005). Up-Scaling Pro-Poor ICT-Policies and Practices. Berne: Swiss Agency for Development and Cooperation (SDC).

Giesbers, B., Rienties, B., Gijselaers, W. H., Segers, M., \& Tempelaar, D. T. (2009). Social presence, Web videoconferencing and learning in virtual teams. Industry and Higher Education, 23(4), 301-309.

Global Development Learning Network. (2005). GDLN Toolkit. Retrieved April, 2007

Golden-Biddle, K., \& Locke, K. (1999). Appealing work: An investigation of how ethnographic texts convince. Organization Science, 4(4), p. 595 (522 pages)

Goldkuhl, G. (2012). Pragmatism vs interpretivism in qualitative information systems research. European Journal of Information Systems, 21(2), 135-146. 
Gray, V., Magpantay, E., Thompson, H., de Ridder, J., \& Southwood, R. (2006). World Telecommunication/ICT Development Report 2006 - Measuring ICT for Social and Economic Development. Geneva, Switzerland

Greenberg, A. (2004). Navigating the Sea of Research on Videoconferencing-Based Distance Education: A Platform for Understanding Research into the Technology's Effectiveness and Value (White paper): Wainhouse Research LLC.

Gudykunst, W. (2002). Cross-Cultural Communication: Introduction. In W. Gudykunst $\&$ B. Mody (Eds.), Handbook of international and intercultural communication (2nd ed.). Thousand Oaks, CA: Sage Publications.

Gudykunst, W., \& Kim, Y. Y. (1997). Communicating with Strangers: An Approach to Intercultural Communication ( $3 \mathrm{~d}$ ed.): McGraw-Hill.

Gump, S. E. (2006). Bridging the Culture Gap: A Practical Guide to International Business Communication/Cultural Intelligence: A Guide to Working with People from Other Cultures. The Journal of Language for International Business, 17(1), 134.

Guo, Z., \& D'Ambra, J. (2003, 10-13 July 2003). Understanding the Role of National Culture on Communication Media Choice Behavior: A Cross-Cultural Comparison within a Multinational Organizational Setting. Paper presented at the Pacific Asia Conference on Information Systems, Adelaide, South Australia.

Gupta, A. K., \& Govindarajan, V. (2002). Cultivating a Global Mindset. The Academy of Management Executive, 16(1), 116-126.

Guzley, R., Avanzino, S., \& Bor, A. (2001). Simulated Computer-Mediated/VideoInteractive Distance Learning: A Test of Motivation, Interaction Satisfaction, Delivery, Learning \& Perceived Effectiveness Journal of Computer-Mediated Communication, 6(3).

Haggis, T. (2006). Pedagogies for diversity: retaining critical challenge amidst fears of 'dumbing down'. Studies in Higher Education, 31(5), 521-535.

Hall, E. T. (1959). The silent language. Greenwich, CT: Fawcett.

Hall, E. T. (1976). Beyond culture (1st ed.). Garden City, N.Y.: Anchor Press.

Hambleton, R. K. (1994). Guidelines for Adapting Educational and Psychological Tests: A Progress Report. European Journal of Psychological Assessment, 10, 229-244.

Hansen, C. D. (1995). Occupational cultures: Whose frame are we using? The Journal for Quality and Participation, 18(3), 60-64.

Hauber, J., Regenbrecht, H., Hills, A., Cockburn, A., \& Billinghurst, M. (2005, 21-23rd September, 2005). Social Presence in Two- and Three-dimensional

Videoconferencing. Paper presented at the The Eight International Workshop on Presence, London, UK.

Hedestig, U., \& Kaptelinin, V. (2005). Facilitator's Roles in a Videoconference Learning Environment. Information Systems Frontiers, 7(1), pp. 71-83.

Hirschheim, R., \& Klein, H. K. (1989). Four paradigms of information systems development. Communications of the ACM, 32(10), pp. 1199-1216.

Hobart, M. (1993). Introduction: the growth of ignorance. In M. Hobart (Ed.), An anthropological critique of development: the growth of ignorance (pp. 1-30). London, New York: Routledge, .

Hofstede, G. (1980). Culture's consequences: International Differences in Work-related Values. Beverly Hills, CA: Sage Publications.

Hofstede, G. (1991). Cultures and organizations : software of the mind. London; New York: McGraw-Hill.

Hofstede, G. (1998). Attitudes, values and organizational culture: Disentangling the concepts. Organization Studies, 19(3), 477-493. 
Hofstede, G. (2001). Cultures consequences: Comparing values, behaviors, institutions and organizations across nations: Thousand Oaks, CA: Sage.

Hofstede, G. (2002). Dimensions do not exist: A reply to Brendan McSweeney. Human Relations, 55(11), 1355.

Hofstede, G., Neuijen, B., Ohayv, D. D., \& Sanders, G. (1990). Measuring organizational cultures: a qualitative and quantitative study across twenty cases. Administrative Science Quarterly, 35(2), 286-316.

Hofstede, G. H. (1997). Cultures and organizations : software of the mind (Rev. ed.). New York: McGraw-Hill.

Hoft, N. (1996). Developing a Cultural Model. In E. M. del Galdo \& J. Nielsen (Eds.), International User Interfaces (pp. 41-73). New York, NY: John Wiely \& Sons, Inc, .

Holden, N., \& Gale, A. (1993). Western-sponsored training programmes: Russian managers' expectations. Leadership \& Organization Development Journal., 14(6), p. 24 (26 pages).

Hubbert, K., Gudykunst, W., \& Guerrero, S. (1999). Intergroup communication over time. International Journal of Intercultural Relations, 23(1), 13-46.

Huijser, H., Bedford, T., \& Bull, D. (2008). OpenCourseWare, Global Access and the Right to Education: Real access or marketing ploy? International Review of Research in Open and Distance Learning, 9(1), 1-13.

Hunter, G. (2001). Cross Cultural Research Issues (Editorial). Journal of Global Information Management, 8(9), p. 3.

Jacobson, T. L., \& Storey, D. J. (2004). Development Communication and Participation: Applying Habermas to a Case Study of Population Programs in Nepal Communication Theory, 14(2), pp. 99-121.

Jandt, F. E. (2001). Intercultural Communication: An Introduction (3rd ed.). Thousand Oaks, CA: Sage Publications.

Jarvenpaa, S. L., Knoll, K., \& Leidner, D. E. (1998). Is Anybody Out There? Journal of Management Information Systems, 14(4), 29-64.

Jarvenpaa, S. L. L., Karl Reiner; Takeda, Yoko; Tuunainen, Virpi Kristiina. (2003). Mobile Commerce at Crossroads. Communications of the ACM, 46(12), 41-44.

JICA-Net. (2009). Partnership between JICA-Net and GDLN/TDLC at a glance. Retrieved August, 2009, from http://jicanet.jica.go.jp/en2/guide/gdln_tour/collabo_01.html

Johansen, R., Vallee, J., \& Spangler, K. (1979). Electronic Meetings: Technological Alternatives and Social Choices. Reading, MA: Addison-Wesley.

Jonjoubsong, L. (2008). An Integrated Knowledge Management Model for Community Enterprises: A Case Study of a Rural Community Enterprise in Thailand. Victoria University of Wellington, Wellington.

Kahane, E. (2000). Thoughts on the Quality of Interpretation [Electronic Version]. Communicate! Retrieved 16 February 2009 from http://www.aiic.net/ViewPage.cfm/article117.htm.

Kanuka, H., Collett, D., \& Caswell, C. (2002). University Instructor Perceptions of the Use of Asynchronous Text-Based Discussion in Distance Courses. American Journal of Distance Education, 16(3), 151-167.

Karahanna, E. (1995). Electronic media in the workplace: An integrated model of media choice. The Journal of Management Systems, 7(2), 1-28.

Karahanna, E., Evaristo, R., \& Srite, M. (2002). Methodological issues in MIS crosscultural research. Journal of Global Information Management, 10(1), p. 48 (48 ).

Karahanna, E., Evaristo, R., \& Srite, M. (2005). Levels of culture and individual behavior: An integrative perspective. Journal of Global Information Management, 13(2), 1-20. 
Keesing, R. M. (1974). Theories of Culture. Annual Review of Anthropology, 3, 73-97. Keil, M. (2000). A Cross-Cultural Study on Escalation of Commitment Behavior in Software Projects. MIS Quarterly, 24(2), 299.

Keil, M., \& Johnson, R. D. (2002). Feedback channels: Using social presence theory to compare voice mail to e-mail. Journal of Information Systems Education, 13(4).

Keystone Conference. (2005, 2005). Interactive VideoConferencing: Igniting Opportunities for Learning. Retrieved April, 2007

Kim, B., \& Oh, H. (2000). An exploratory inquiry into the perceived effectiveness of a global information system. Information Management \& Computer Security, 8(3), p. 144 (110 pages).

Kim, K.-J., \& Bonk, C. (2002). Cross-cultural comparisons of online collaboration. Journal of Computer-Mediated Communication, 8(1).

Kim, Y. (1984). Searching for Creative Integration. In W. K. Gudykunst \& Y. Y. Kim (Eds.), Methods for Intercultural communication research (Vol. 8). Beverly Hills: Sage Publications.

Kim, Y. (2005). Inquiry in Intercultural and Development Communication Journal of Communication, 55(3), pp. 554-577.

Kim, Y. Y. (1984). Searching for creative integration. In W. K. Gudykunst, Young Yun (Ed.), Methods for intercultural communication research (Vol. 8). Beverly Hills: Sage Publications.

Kittler, M. G., Rygl, D., \& Mackinnon, A. (2011). Beyond culture or beyond control? Reviewing the use of Hall's high-/low-context concept. International Journal of Cross Cultural Management, 11(1), 63-82.

Klein, H. K., \& Myers, M. D. (1999). A Set of Principles for Conducting and Evaluating Interpretive Field Studies in Information Systems. MIS Quarterly., 23(1).

Knipe, D., \& Lee, M. (2002). The Quality of Teaching and Learning via Videoconferencing. British Journal of Educational Technology 33(3), 301-311.

Kolosov, V. A., Galkina, T. A., \& Krindach, A. D. (2003). Territorial Identity and Interethnic Relations. Russian Social Science Review, 44(2), 41-66.

Koro-Ljungberg, M. (2004). Impossibilities of reconciliation: validity in mixed theory projects. Qualitative Inquiry, 10(4), 601-621.

Kotter, J. P., \& Heskett, J. L. (1992). Corporate Culture and Performance. New York, NY: Free Press.

Kreuter, M. W., \& McClure, S. M. (2004). The Role of Culture in Health Communication. Annual Review of Public Health, 25, 439-455.

Kurz, I. (2001). Conference Interpreting: Quality in the Ears of the User. Meta: Translators' Journal, 46(2), 394-409.

Kurz, I. (2003). Physiological stress during simultaneous interpreting: a comparison of experts and novices. Interpreters' Newsletter, 51-68.

Larmour, P. (2008). Corruption and the concept of 'Culture': Evidencefrom the Pacific Islands. Crime Law and Social Change, 49(3), 225-239.

Lasswell, H. (1948). The structure and function of communication in society. In L. Bryson (Ed.), The communication of Ideas (pp. 32-51). New York: Harper.

Lee, A. S., \& Baskerville, R. L. (2003). Generalizing generalizability in information systems research. Information Systems Research, 14(3), 221-243.

Lee, I., Choi, B., Kim, J., Hong, S.-J., \& Tam, K. Y. (2004). Cross-cultural Comparison for Cultural Aspects of Mobile Internet: Focusing on Korea and Hong Kong. Paper presented at the 10th Americas Conference on Information Systems, New York. 
Lee, Z., \& Lee, Y. (2003). Cultural Implications of Electronic Communication Usage: A Theory-based Empirical Analysis. Paper presented at the 24th International Conference on Information Systems.

Leeds-Hurwitz, W. (1990). Notes in the History of Intercultural Communication: The Foreign Service Institute and the Mandate for Intercultural Training. Quarterly Journal of Speech, 76(3), pp. 262-281

Leidner, D., \& Kayworth, T. (2006). A review of culture in information systems research: towards a theory of information technology culture conflict. MIS Quarterly, 30(2), pp. 357-399.

Leonard, K. M., VanScotter, J. R., \& Pakdil, F. (2009). Culture and Communication : Cultural Variations and Media. Administration \& Society, 41(7), 850-877.

Lexcen, F. J., Hawk, G. L., Herrick, S., \& Blank, M. B. (2006). Use of Video Conferencing for Psychiatric and Forensic Evaluations Psychiatric Services, 57(5), 713.

Limaye, M. R., \& Victor, D. A. (1991). Cross-cultural business communication research: state of the art and hypotheses for the 1990s. The Journal of Business Communication, 28(3), 277-299.

Linton, J. D. (2002). Implementation research: state of the art and future directions. Technovation 22(2), pp. 65-79

Lorenzo, G. (2002). World Bank's Global Development Learning Network: Sharing Knowledge Electronically Between Nations to "Fight Poverty". USDLA (United States Distance Learning Association) Journal 16(3).

Markus, M. L. (1994). Electronic Mail as the Medium of Managerial Choice. Organization Science, 5(4), 502-527.

Martin, J. N., \& Nakayama, T. K. (1999). Thinking dialectically about culture and communication. Communication Theory, 9(1), p. 1 (25 pages)

Martin, K. (2005). Seeing is believing: the role of videoconferencing in distance learning. British Journal of Educational Technology, 36(3), 397.

Martinsons, M. G., \& Davison, R. (2003). Cultural Issues and IT Management: Looking Ahead. IEEE Transactions on Engineering Management, 50(1), 113-117.

Martz, W. B., Jr. and Venkateshwar Reddy. (2005). Looking for Indicators of Media Richness Theory in Distance Education. Paper presented at the 38th International Conference on Social Sciences, Hawaii.

Marziali, E., Donahue, P., \& Crossin, G. (2005). Caring for Others: Internet Health Care Support Intervention for Family Caregivers of Persons With Alzheimer's, Stroke, or Parkinson's Disease. Families in Society, 86(3), 375.

Matveev, A. V., \& Milter, R. G. (2004). The value of intercultural competence for performance of multicultural teams. Team Performance Management, 10(5/6).

McClymont, K. (2009, 10 April 2009). Fiji military coup was illegal, rules court. The Sydney Morning Helarld Retrieved 15 August 2009, 2009, from http://www.smh.com.au/news/world/fiji-military-coup-was-illegal-rulescourt/2009/04/09/1239222991945.html

McCombs, G., Ufnar, J., \& Shepherd, V. (2007). The Virtual Scientist: connecting university scientists to the K-12 classroom through videoconferencing. Advances in Physiology Education, 31(1), 62-66.

McCoy, S., Galletta, D. F., \& King, W. R. (2005). Integrating National Culture into IS Research: The Need for Current Individual Level Measures. Communications of AIS, , 2005(15), p211-224, 214p.

McFarlane, C. (2006). Knowledge, learning and development: a post-rationalist approach. Progress in Development Studies, 6(4), 287-305. 
McGuire, W. (1989). Theoretical foundations of campaigns. In R. Rice \& C. Atkin (Eds.), Public Communication Campaigns (pp. 43-65). Newbury Park, CA: Sage Publications.

Mehta, L. (1999). From Darkness to Light? Critical Reflections on the World Development Report 1998/99. Journal of Development Studies, 36(1), 151-161.

Melkote, S., \& Steeves, L. (2001). Communication for development in the Third World : theory and practice for empowerment / (2nd ed.). London: Sage.

Merrick, S. (2005). Videoconferencing for Primary and Secondary Schools - Where Are We? (White paper): Tandberg.

Meyerson, D., Weick, K. E., \& Kramer, R. M. (1996). Swift trust and temporary groups. In R. M. Kramer \& T. R. Tyler (Eds.), Trust in Organizations: Frontiers of Theory and Research (pp. 166-195). Thousand Oaks, CA: Sage Publications.

Michailova, S. (2000). Contrasts in culture: Russian and Western perspectives on organizational change. The Academy of Management Executive, 14(4), 99-112.

Middlehurst, R., \& Woodfield, S. (2006). Quality Review in Distance Learning: Policy and Practice in Five Countries. Tertiary Education and Management, 12(1).

Milobsky, D., \& Galambos, L. (1995). The McNamara Bank and Its Legacy, 19681987. Business and Economic History, 24(2).

Miranda, S. M., \& Saunders, C. S. (2003). The social construction of meaning: An alternative perspective on information sharing. Information Systems Research, 14(1).

Moore, H. (1996). The changing nature of anthropological knowledge: an introduction. In H. Moore (Ed.), The future of anthropological knowledge: Routledge.

Moridani, M. (2007). Asynchronous Video Streaming vs. Synchronous Videoconferencing for Teaching a Pharmacogenetic Pharmacotherapy Course. American Journal of Pharmaceutical Education, 71(1), 1-10.

Morris, N. (2003). A Comparative Analysis of the Diffusion and Participatory Models in Development Communication. Communication Theory, 13(2), pp. 225-248.

Mosier, J. N., \& Tammaro, S. (1994). Video Teleconference Use Among Geographically Dispersed Work Groups: A Field Investigation of Usage Patterns and User Preferences. Journal of Organizational Computing \& Electronic Commerce, 4(4), p. 343 (323 pages).

Muzykantsky, A. (2005). A Yardstick for Russia. Russia in Global Affairs, 3(3), 8-18.

Myers, M., \& Newman, M. (2007). The qualitative interview in IS research: Examining the craft Information and Organization, 17(1), 2-26.

Myers, M. D. (1997). Qualitative Research in Information Systems. MISQ Discovery.

Myers, M. D., \& Tan, F. B. (2002). Beyond models of national culture in information systems research. Journal of Global Information Management, 10(1), p. 24 (29).

Nandhakumar, J., \& Jones, M. (1997). Too close for comfort? Distance and engagement in interpretive information systems research. Information Systems Journal, 7(2), pages 109-131.

Nardon, L., \& Steers, R. M. (2008). The New Global Manager: Learning Cultures on the Fly. Organizational Dynamics, 37(1).

Naugle, L. M. (2002). Distributed choreography: A video-conferencing environment. PAJ : a Journal of Performance and Art, 24(2), 56.

Neuhauser, C. (2002). Learning Style and Effectiveness of Online and Face-to-Face Instruction. American Journal of Distance Education, 16(2).

Ngwenyama, O. K., \& Lee, A. S. (1997). Communication richness in electronic mail: critical social theory and the contextuality meaning. MIS Quarterly, 21(2), 145.

Nonaka, I., Takeuchi, H. (1995). The Knowledge-creating Company: How Japanese Companies Create the Dynamics of Innovation. New York, NY: Oxford University Press. 
O'Connail, B. (1993). Conversations Over Video Conferences: An Evaluation of the Spoken Aspects of Video-Mediated Communication. Human-Computer Interaction, 8(4), p. 389.

O'Dowd, R. O. A. V., Iss. 2; p. 144. (2007). Evaluating the outcomes of online intercultural exchange. ELT Journal, 61(2), 144-152.

OECD. (2006). Selected Statistics on Higher Education (Background Report). Athens: Organisation for Economic Co-operation and Development.

Olaniran, B. A. (2009). Organizational Communication: Assessment of Videoconferencing as a Medium for Meetings in the Workplace. International Journal of Technology and Human Interaction, 5(2), 63-84.

Olsen, F. (2003). Videoconferencing With Some Life to It: The U. of Arizona Finds That Pricey Technology Attracts Distance-Education Students. The Chronicle of Higher Education, 49(43).

Olson, G., \& Olson, J. (2000). Distance matters. Human-Computer interaction, 15, pp. 139-178.

Olson, J. S., \& Olson, G. M. N. Y. D. V., Iss. 9. (2003). Culture Surprises in Remote Software Development Teams. ACM Quеие, 1(9), 52-59.

Orlikowski, W. J., \& Baroudi, J. J. (1991). Studying Information Technology in Organizations: Research Approaches and Assumptions. Information Systems Research., 2(1).

Pagram, P., \& Pagram, J. (2006). Issues in E-Learning: A Thai Case Study. Electronic Journal on Information Systems in Developing Countries, 26(6), 1-8.

Panteli, N., \& Dawson, P. (2001). Video conferencing meetings: Changing patterns of business communication New Technology, Work and Employment, 16(2).

Patrick, A. S. (1999). The Human Factors of MBone Videoconferences:

Recommendations for Improving Sessions and Software. Journal of Computer Mediated Communication, 4(3).

Patton, M. Q. (1999). Enhancing the Quality and Credibility of Qualitative Analysis. Health Services Research, 34(5), p. 1189.

Pauleen, D. J., Evaristo, R., Davison, R. M., \& Ang, S. e. a. (2006). Cultural Bias in Information Systems Research and Practice: Are You Coming From the Same Place I Am? . Communications of the Association for Information Systems. , $17(1)$.

Pauleen, D. J., \& Murphy, P. (2005). In Praise of Cultural Bias. MIT Sloan Management Review., 46(2), p. 21.

Peltokorpi, V., \& Clausen, L. (2011). Linguistic and cultural barriers to intercultural communication in foreign subsidiaries. Asian Business \& Management, 10(4), 509-528.

Peszynski, K. J., \& Yoong, P. (2002). The role of information and communication technology in the urgent decision-making process: A work in progress report: Deakin University, Australia.

Peterson, C. (2004). Making interactivity count: best practices in video conferencing. Journal of Interactive Learning Research, 15(1), 63-75.

Peterson, D. K., Kim, C., \& Kim, J. H. (2003). Perceptions of Information Systems objectives: A comparison of IS professionals from the United States and Korea. Journal of Global Information Technology Management, 6(2).

Peuch, J.-C. (2007). Russia: Putin's Repatriation Scheme Off To Slow Start [Electronic Version]. Radio Free Europe Radio Liberty. Retrieved February 1, 2009 from http://www.rferl.org/content/Article/1075988.html.

Piccoli, G., Ahmad, R., \& Ives, B. (2001). Web-based virtual learning environments: A research framework and a preliminary assessment of effectiveness in basic IT skills training. MIS Quarterly., 25(4), p. 401 (426 pages). 
Pöchhacker, F. (1999). Review of "La evaluación de la calidad en interpretación simultánea: La importancia de la comunicación no verbal" by Ángela Collados Ais. Interpreting, 4(2), 219-230.

Prasad, A. (2002). The contest over meaning: Hermeneutics as an interpretive methodology for understanding texts. Organizational Research Methods, 5(1), p. 12 (22 pages).

Price, J., \& Sapci, H. (2007). Law \& Psychiatry: Telecourt: The Use of Videoconferencing for Involuntary Commitment Hearings in Academic Health Centers. Psychiatric Services, 58(1), 17.

Profile of New Zealander Responses, Ethnicity Question: 2006 Census. (2006).). Wellington: Statistics New Zealand.

Rees, C. S., \& Stone, S. (2005). Therapeutic Alliance in Face-to-Face Versus Videoconferenced Psychotherapy. Professional Psychology : Research and Practice, 36(6), 649.

Rice, R. E. (1993). Media Appropriateness. Using Social Presence Theory to Compare Traditional and New Organizational Media Human Communication Research, 19(4), 451-484.

Richman, A., Noble, K., \& Johnson, A. (2002). When the workplace is many places: The extent and nature of off-site work today. Executive Summary. Retrieved April, 2007, from http://www.abcdependentcare.com/docs/ABC_Executive_Summary_final.pdf

Riley, P. (2009). Video-conferenced music teaching: challenges and progress. Music Education Research, 11(3), 365-375.

Robinson, M. (1998). Corruption and development: An introduction. European Journal of Development Research, 10(1), 1-14

Rogers, E. (1962). Diffusion of Innovations New York: The Free Press.

Rogers, E. (1976). Communication and Development: The passing of the dominant paradagm. In E. Rogers (Ed.), Communication and Development:Critical Perspectives (pp. 67-86). Beverly Hills, CA: Sage Publications.

Rubin, H. J. (2005). Qualitative interviewing : the art of hearing data (2nd ed.). Thousand Oaks, California Sage Publications.

Rutkowski, A., Vogel, D., Bemelmans, T., \& van Genuchten, M. (2002). Group Support Systems and Virtual Collaboration: The HKNET Project Group Decision and Negotiation, 11(2), 101.

Ryan, C. (2003). African Connectivity No Guarantee of Economic Upliftment. Government Technology International.

Sablonnière, R., Tougas, F., \& Lortie-Lussier, M. (2009). Dramatic Social Change in Russia and Mongolia: Connecting Relative Deprivation to Social Identity. Journal of Cross-Cultural Psychology, 40, 327-348.

Sahay, S., \& Avgerou, C. (2002). Introducing the Special Issue on Information and Communication Technologies in Developing Countries. The Information Society, 18(2), 73-76.

Samovar, L. A., Porter, R. E., \& Jain, N. C. (1981). Understanding intercultural communication. Belmont, CA: Wadsworth.

Sandberg, J. (2005). How Do We Justify Knowledge Produced Within Interpretive Approaches? Organizational Research Methods, 8(1), p. 41 (28 pages).

Selim, H. M. (2005). Videoconferencing-Mediated Instruction: Success Model. Journal of Distance Education Technologies, 3(1), 62-80.

Sellen, A. (1995). Remote Conversations: The Effects of Mediating Talk With Technology. Human-Computer Interaction, 10(4), p. 401. 
Servaes, J. (2007). Harnessing the UN System Into a Common Approach On Communication for Development. The International Communication Gazette, 69(6), 483-507.

Servaes, J., \& Malikhao, P. (2004, 6 - 9 September). Communication and Sustainable Development: Focus on Sustainable Development. Paper presented at the 9th United Nations Roundtable on Communication for Development, Rome, Italy.

Setzer, C., \& Lewis, L. (2006). Distance Education Courses for Public Elementary and Secondary School Students: 2002-03. Education Statistics Quarterly, 7(1).

Shannon, C. E., \& Weaver, W. (1949). The mathematical theory of communication. Urbana, IL: University of Illinois Press.

Sherry, L. (1996). Issues in Distance Learning. . International Journal of Educational Telecommunications, , 1(4), 337-365.

Shkaratan, O. (2007). Social Policy: Target-the New Middle Class. Social Sciences, $38(2), 88-103$.

Shlesinger, M. (1995). Shifts in Cohesion in Simultaneous Interpreting. The Translator, l(2), 193-214.

Shome, R., \& Hegde, R. S. (2002). Postcolonial approaches to communication: charting the terrain, engaging the intersections. Communication Theory, 12(3), p. 249 (222).

Simpson, S., Bell, L., Britton, P., \& Mitchell, D. (2006). Does Video Therapy Work? A Single Case Series of Bulimic Disorders. European Eating Disorders Review, 14(4), 226.

Singer, M. R. (1998). The role of culture and perception in communication. In G. R. Weaver (Ed.), Culture, Communication and Conflict: Readings in Intercultural Relations. New York: Simon \& Schuster.

Singh, J. P. (2002). Communication Technology and development. In W. Gudykunst \& B. Mody (Eds.), Handbook of international and intercultural communication (2nd ed., pp. 479-497). Thousand Oaks, CA: Sage Publications.

Starke-Meyerring, D. A., Deborah. (2006). Building a Shared Virtual Learning Culture. Business Communication Quarterly, 69(1), 25-49.

Statistics New Zealand. (2007). QuickStats About Culture and Identity [Electronic Version]. New Zealand's 2006 Census of Population and Dwellings. Retrieved 11 August 2009 from http://www.stats.govt.nz/ /media/Statistics/Publications/Census/2006\%20Censu s\%20reports/QuickStats\%20About\%20A\%20Subject/QuickStats\%20About\%20 Culture\%20and\%20Identity/qstats-about-culture-and-identity-2006-census.ashx.

Steeves, L. H. (2001). Liberation, Feminism, and Development Communication. Communication Theory, 11(4), 397-414.

Stiglitz, J. E. (2004). Globalization and growth in emerging markets. Journal of Policy Modeling, 26(4), 465-484.

Stödberg, U., \& Orre, C. J. (2010). It's not all about video-conferencing. Campus-Wide Information Systems, 27(3), 109-117.

Stonebraker, P. W., \& Hazeltine, J. E. (2004). Virtual learning effectiveness: An examination of the process. The Learning Organization, 11(2/3), p. 209

Straub, D., Loch, K., Evaristo, R., Karahanna, E., and Srite, M. (2002). Toward a Theory-Based Measurement of Culture. Journal of Global Information Management, 10(1), pp. 13-23.

Straus, S. G., Miles, J. A., \& Levesque, L. L. (2001). The effects of videoconference, telephone, and face-to-face media on interviewer and applicant judgments in employment interviews. Journal of Management, 27(3), p. 363. 
Suh, K. S. (1999). Impact of communication medium on task performance and satisfaction: An examination of media-richness theory. Information \& Management, 35(5), 295-312.

Taft, R. (1981). The Role and Personality of the Mediator. In S. Bochner (Ed.), The Mediating Person: Bridges between Cultures (pp. 53-88). Cambridge: Schenkman.

Tajfel, H., \& Turner, J. (1985). The social identity theory of intergroup behavior. In S. Warchel \& W. Austin (Eds.), Psycology of intergroup relations (2nd ed., pp. 724). Chicago: Nelson-Hall.

Tan, B. C. Y. S., H. J.; Keil, M. (2003). Reporting Bad News about Software Projects: Impact of Organizational Climate and Information Asymmetry in an Individualistic and Collectivist Culture. IEEE Transactions on Engineering Management, 50(1), 65-77.

Tang, J. C., \& Isaacs, E. (1992). Why do users like video? Computer Supported Cooperative Work (CSCW), 1(3), pp. 163-196.

The World Bank. (2007, April 2007). Development Communication. Retrieved April, 2007, from http://go.worldbank.org/BXYX66PE10

Tokyo Development Learning Center. (2004). World Bank GDLN and JICA-Net. Retrieved August, 2009, from http://www.jointokyo.org/en/partners/C22/

Tool, M. R. (1977). Beyond culture (book review). Journal of Economic Issues, 11, 899-901.

Treadway Johnson, M., \& Wiggins, E. C. (2006). Videoconferencing in Criminal Proceedings: Legal and Empirical Issues and Directions for Research. Law \& Policy, 28(2), 211.

Turner, J. (1985). Social categorisation and the self-concept: A social cognitive theory of group behavior. In E. J. Lawler (Ed.), Advances in Group Processes (Vol. 2). Greenwich, CT: IAI Press.

Valacich, J. S., Mennecke, B. E., Wachter, R. M., \& Wheeler, B. C. (1994). Extensions to media richness theory: a test of the task-Media fit hypothesis. Paper presented at the 27th Annual Hawaii International Conference on System Sciences.

Van Ast, P. (2005). Supporting community carers via videoconferencing. Journal of Telemedicine and Telecare, 11(Suppl. 2), 96-97.

van de Vijver, F., \& Hambleton, R. K. (1996). Translating Tests: Some Practical Guidelines. European Psychologist, 1(2), 89-99.

van de Vijver, F., \& Leung, K. (1997). Methods and Data Analysis for Cross-Cultural Research. Thousand Oaks, CA: SAGE Publications.

van den Berg, P. T. W., Celeste P.M.. (2004). Defining, Measuring, and Comparing Organisational Cultures. Applied Psychology: An International Review, 53(4), 570-582.

Visson, L. (1999). From Russian into English: An Introduction to Simultaneous Interpretation, (Second Edition (Russian Edition) ed.). Newburyport, MA: Focus Publishing.

Vries, E., \& Roest, D. (1999). Case Research in IS: State of Affairs. Paper presented at the 7th European Conference on Information Systems, Copenhagen.

Waisbord, S. (2001). Family Tree of Theories, Methodologies and Strategies in Development Communication: Convergences and Differences. New York: The Rockefeller Foundation.

Waiti, P. (2005). Evaluation of Kaupapa Ara Whakawhiti Mätauranga (KAWM). Wellington: Ministry of Education.

Waits, T., \& Lewis, L. (2004). Distance Education at Degree-Granting Postsecondary Institutions: 2000-2001. Education Statistics Quarterly, 5(3). 
Walsham, G. (1995). Interpretive case studies in IS research: Nature and method European Journal of Information Systems., 4(2).

Walsham, G. (2002). Cross-cultural software production and use: a Structurational analysis. MIS Quarterly, 26(4), p359-380, 322p.

Walsham, G. (2006). Doing interpretive research. European Journal of Information Systems, 15(3), 320-330.

Warkentin, M. E., Sayeed, L., \& Hightower, R. (1997). Virtual teams versus face-toface teams: An exploratory study of a Web-based conference system Decision Sciences, 28(4), pp. 975-996.

Weaver, C., \& Leiteritz, R. J. (2005). "Our Poverty Is a World Full of Dreams:" Reforming the World Bank. Global Governance, 11(3), 369-388.

Weber-Fahr, M. (2006, May 12-14 2006). GDLN Around the World - in 2006: Evolving Communities, Broader Constituencies, Wider Reach. Paper presented at the 12th EAPA Regional Meeting, Bangkok, Thailand.

Webster, J. (1998). Desktop Videoconferencing: Experiences of Complete Users, Wary Users, and Non-Users. MIS Quarterly, 22(3), pp. 257-286 (230 pages).

Wiggins, E. C. (2006). The Courtroom of the Future is Here: Introduction to Emerging Technologies in the Legal System. Law \& Policy, 28(2), 182.

Wilkins, K. (2002). International Development Communication: Proposing a Research Agenda for a New Era. In Handbook of international and intercultural communication (pp. 537-550). Thousand Oaks, CA: Sage Publications.

Wilkins, K., \& Waters, J. (2000). Current discourse on new technologies in development communication. Media Development, 1, 57-60.

Wilkins, K. G. (1999). Development discourse on gender and communication in strategies for social change. Journal of Communication, 49(1), p. 46 (23 pages).

Wilkins, K. G., \& Mody, B. (2001). Reshaping development communication: Developing communication and communicating development. Communication Theory, 11(4), p. 385 (312 pages).

Williamson, D. (2002). Forward from a critique of Hofstede's model of national culture. Human Relations, 55(11), 1373.

Wilson, A. M. (1997). The nature of corporate culture within a service delivery environment. International Journal of Service Industry Management, 8(1), 87102.

Wilson, A. M. (2001). Understanding organisational culture and the implications for corporate marketing. European Journal of Marketing, 35(3/4), 353.

Wilson, E. V., Morrison, J. P., \& Napier, A. M. (1997/1998). Perceived effectiveness of computer-mediated communications and face-to-face communications in student software development teams The Journal of Computer Information Systems, $38(2)$.

Wisher, R. A., \& Curnow, C. K. (2003). Handbook of Distance Education. Mahwah, NJ Lawrence Erlbaum Associates.

Wolfe, M. (2007). Broadband videoconferencing as a knowledge management tool. Journal of Knowledge Management, 11(2), 118-138.

World Bank. (1999). World Development Report: Knowledge for Development. Oxford and New York: Oxford University Press for the World Bank.

World Bank. (2006). Global Development Learning Network. from http://www.gdln.org/

World Bank. (2008). Using Knowledge to Improve Development Effectiveness. Washington DC: The World Bank.

World Bank. (2009a). China Global Development Learning Network. Retrieved August, 2009, from <http://go.worldbank.org/ZGT90IRC10> 
World Bank. (2009b). Global Development Learning Network. Retrieved August, 2009, from http://www.gdln.org/

World Bank. (2009c). World Bank Institute. Retrieved August, 2009, from http://wbi.worldbank.org/wbi/

World Bank, Food and Agriculture Organization of the United Nations, \& Communication Initiative. (2006). Rome Consensus, World Congress on Communication for Development: Lessons, Challenges, and the Way Forward. Washington, DC: World Bank.

Yao, K. (2003). The Chances for Success of the Francophone Centers for Distance Education of the GDLN Network: The Case of the Centre d'Education à Distance de Côte d'Ivoire. African and Asian Studies, 2(4).

Yazici, A., Altas, I., \& Demiray, U. (2001). Distance Education on the Net: A Model for Developing Countries. Turkish Online Journal of Distance Education, 2(2), 24-35.

Yin, R. K. (1999). Enhancing the Quality of Case Studies in Health Services Research. Health Services Research, 34(5), p. 1209 (1216 pages).

Yin, R. K. (2003). Case Study Research: Design and Methods (3d ed.). Thousand Oaks, London, New Delhi: Sage Publications.

Zaharna, R. S. (2000). Intercultural communication and international public relations: Exploring parallels. Communication Quarterly, 48(1), p. 85 (16).

Zorin, V. (2004). Some aspects of implementing state nationalities policy in light of 2002 All-Russia Population Sensus results. Paper presented at the The Russian in the Mirror of Statistics: the All-Russia Population Census 2002, Moscow. 
Annex A: Information sheet for interviewee

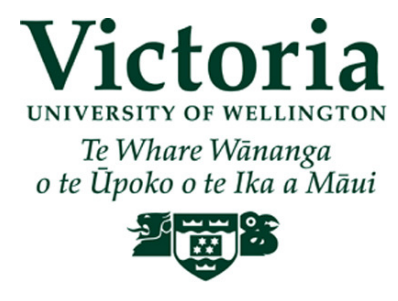

SCHOOL OF INFORMATION MANAGEMENT

Information Sheet for Interviewee

\section{Project Title: Understanding the Effectiveness of Cross-Cultural Video- mediated Communication}

Date

Dear

This letter follows up my recent e-mail in which I expressed my interest in conducting an interview with you regarding your participation in a videoconferencing activity. Prior to conducting the proposed interview, Victoria University of Wellington where I am doing my $\mathrm{PhD}$ studies require that I obtain your written informed consent.

\section{Purpose of Research}

This research project is conducted for my PhD degree in Information Systems. The aim of the project is to study the effectiveness of cross-cultural videomediated communication as perceived by participants of videoconferences and identify the critical factors that influence their perceptions. As a researcher, I am keen to understand the process of cross-cultural communication that occurs via videoconferencing and learn how the cultural differences of participants, among other factors, influence the effectiveness of this technology-mediated interaction. The study will propose a framework for evaluation of effectiveness of video-mediated cross-cultural communication. The study will be conducted in the context of the Global Development Learning Network (GDLN) as an example of an international videoconferencing network.

This research will involve observation of videoconferencing events, interviews with participants, facilitators, moderators, presenters and GDLN staff in six Distance Learning Centres - in Canberra (Australia), Ulaanbaatar (Mongolia), Moscow (Russian Federation) and Wellington (New Zealand) - all affiliated to the network. Interview questions will be related to the issues of cultural differences and other factors that affect participants' perceptions about the 
effectiveness of cross-cultural communication that occurs during videoconferencing events. A preliminary set of interview questions is attached.

You may be approached up to four occasions during the process of the project: 1) obtaining consent; 2) conducting actual interview; 3) verifying the content of interview and providing feedback; 4) introducing the summary of research findings. The actual interview will be recorded and transcription of the interview produced in a text format.

\section{Confidentiality}

All raw data will be kept confidential to the researcher, her supervisor, translator and transcriber, though collected, collated and analysed data may be published in academic journals, and presented at conferences. Any information and opinions that you provide will not be attributed to you, and you will not be able to be identified in any way. There will be an opportunity to review any written notes or transcripts of recorded sessions that results from the interviews to ensure factual material is recorded accurately. A copy of the written notes and the transcription of each interview that you participate in will be sent to you for review and feedback. A summary of key findings and a research report will be provided to research participants. Throughout the project, raw data will be kept under password protection and destroyed two years after the completion of the project.

Please feel free to contact my supervisor Dr. Brian Harmer on +64 44635887 or via email brian.harmer@vuw.ac.nz if you require further information on the project or the informed consent requirement.

You have the right to withdraw from this project at any time up until $1^{\text {st }}$ of March 2008 when data entry is expected. Any information already obtained until then will be omitted from the study.

The consent form is attached. It includes a request for permission to taperecord interviews. I will bring a hard copy of the consent form with me to our interview for your signature.

Thank you for your time.

Yours sincerely,

Selenge Nergui

Phone: $\quad$ 04-463 5504 (office)

021-233 7468 (cell)

Email: $\quad$ selenge.nergui@vuw.ac.nz 
Annex B: Consent form

VICTORIA UNIVERSITY OF WELLINGTON

Te Whare Wānanga o te Ûpoko o te Ika a Māui

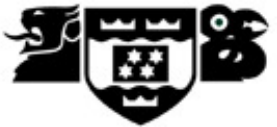

SCHOOL OF INFORMATION MANAGEMENT

\section{Consent to Participate in Research as an Interviewee}

\section{Project Title: Understanding the Effectiveness of Cross-Cultural Video-mediated Communication}

I have been given and have understood an explanation of this research project. I have had the opportunity to ask questions and have them answered to my satisfaction. I understand that I may withdraw myself (or any information I have provided) from the project (before data collection and analysis is complete) without having to give reasons by e-mailing Selenge Nergui or her supervisor at the email address given below by the $1^{\text {st }}$ of March 2008. I understand that if I withdraw from the project, any data I have provided will be returned or destroyed, by the researcher.

I understand that any information I provide during the interview will be tape-recorded and kept confidential to the researcher, her supervisor, and to a translator and transcriber who might be hired. I understand that the information I have provided will be used only for this research project and that any further use will require my written consent. The published results will not use my name, and that no opinions will be attributed to me in any way that will identify me. I understand that the recording of interviews will be erased, two years after the conclusion of the project unless I indicate that I would like them returned to me.

[Please mark with a $\checkmark$ to indicate agreement]

I would like the recordings of my interview returned to me, two years after the completion of the project.

I understand that the data I provide will not be used for any other purpose or released to others without my written consent

I would like to receive a summary of the results of this research when it is completed

I agree to take part in this research

I understand that I can withdraw myself and any information I have provided from the study until 30 February 2008.

Signature:

Name of the participant:

Date:

Please feel free to contact Ms. Selenge Nergui on 021-2337468 (in Wellington) or via email at selenge.nergui@vuw.ac.nz or her supervisor, Lecturer of the School of Information Management of Victoria University of Wellington Dr. Brian Harmer on or 04-463 5887 (in Wellington) via email brian.harmer@vuw.ac.nz for further information on the project or informed consent requirement. 
Annex C: Interview questions

\section{Semi-structured Interview Questions (Facilitator)}

Before the interview, I will introduce myself and briefly explain the purpose of this research I will also explain the meaning of 'Communication effectiveness' in my research ${ }^{7}$.

I will begin my interview with a broad question below:

- Please tell me about the GDLN centre at VUW and your involvement in this network

Based on the facilitator's response, I will proceed with series of main and follow-up questions that will help me to learn more on specific factors:

\section{Cultural differences of participants}

- During GDLN events, people from many cultures gather together to communicate on development topics. Was it the case in the event/s you attended? Please share your experience participating in such cross-cultural communication.

Based on participant's response, I will use follow up questions and probes to get indepth understanding on how cultural differences of participants influence the effectiveness of communication that occurs during videoconference/s.

\section{Facilitation:}

Before proceeding to this issue, I will explain what 'facilitation' means in this context.

- Can you step me through your communication with the facilitator starting from the point when you were approached first (received an invitation to participate in this event) and last (asked to fill in an evaluation form or other after-event follow-up activity).

\section{Content relevance}

- Could you please tell me about the relevance of the topic to the work you do?

Based on the response, I will elicit more follow up questions to learn more about the knowledge obtained by the participant from this event.

\section{Moderation $^{8}$ :}

Before proceeding to this aspect of my interview, I will explain what I mean by 'moderation'

\footnotetext{
${ }^{7}$ Communication effectiveness refers to the extent that individuals are able to minimize misunderstandings (Hubbert, Gudykunst, \& Guerrero, 1999).

${ }^{8}$ The key roles of a moderator for a videoconference include: introduce all speakers, panellists, resource persons and VIP guests; keep the event flowing smoothly by making all the transitions from site to site and person to person; manage the time according to the event agenda; make the event interactive by ensuring that participants are recognized and given the opportunity to speak/interact; facilitate the communication by eliciting questions and comments and providing summaries during and at the end of the session to highlight important points.
} 
- Could you tell me about the moderation during event/s.

\section{Presentation}

- Can you tell me about the presenter(s)'s effectiveness

\section{Translation/interpretation}

- You probably noticed that participants at some sited connected to the videoconference/s communicated with the help of simultaneous interpreters. Please tell me your view on the translator-mediated communication during videoconferences.

\section{Effectiveness of technology}

- What has been your experience with 'technology failures' during the videoconference/c? In your view, how efficiently they were handled?

\section{Other factors}

- Please tell me about other important factors that, in your view, influence the effectiveness of communication during videoconferences.

- Among factors we've discussed, which ones are the most/least important?

\section{Semi-structured Interview Questions (Participant)}

Before the interview, I will introduce myself and briefly explain the purpose of this research I will also explain the meaning of 'Communication effectiveness' in my research.

I will begin my interview with a broad question below:

- Please tell me about your videoconferencing experience/s.

Based on the participant's response, I will proceed with series of main and follow-up questions that will help me to learn more on specific factors:

\section{Cultural differences of participants}

- During GDLN events, people from many cultures gather together to communicate on development topics. Was it the case in the event/s you attended? Please share your experience participating in such cross-cultural communication.

Based on participant's response, I will use follow up questions and probes to get indepth understanding on how cultural differences of participants influence the effectiveness of communication that occurs during videoconference/s. 


\section{Facilitation ${ }^{9}$ :}

Before proceeding to this issue, I will explain what 'facilitation' means in this context.

- Can you step me through your communication with the facilitator starting from the point when you were approached first (received an invitation to participate in this event) and last (asked to fill in an evaluation form or other after-event follow-up activity).

\section{Content relevance}

- Could you please tell me about the relevance of the topic to the work you do?

Based on the response, I will elicit more follow up questions to learn more about the knowledge obtained by the participant from this event.

\section{Moderation:}

Before proceeding to this aspect of my interview, I will explain what I mean by 'moderation'

- Could you tell me about the moderation during event/s.

\section{Presentation}

- Can you tell me about the presenter(s)'s effectiveness

\section{Translation/interpretation}

- You probably noticed that participants at some sited connected to the videoconference/s communicated with the help of simultaneous interpreters. Please tell me your view on the translator-mediated communication during videoconferences.

\section{Effectiveness of technology}

- What has been your experience with 'technology failures' during the videoconference/c? In your view, how efficiently they were handled?

\section{Other factors}

- Please tell me about other important factors that, in your view, influence the effectiveness of communication during videoconferences.

- Among factors we've discussed, which ones are the most/least important?

\footnotetext{
${ }^{9}$ Facilitation at the participating DLCs consists of activities to be carried out before, during and after the videoconferencing event. Preparation would include: identification of individual participants; timely distribution of program outlines and course materials; contracting translators and interpreters; preparing copies of training materials, etc. On the day of the event, the facilitator should welcome and register the participants, briefly explain to them the use of relevant equipment (headsets and microphones) and ensure smooth coordination between participants, moderator, interpreter and technical staff. After the event, the facilitator ensures that evaluation forms are filled and follow up actions carried out.
} 


\section{Annex D: Sample classification of nodes (Russian case)}

\section{Picture 1: Tree nodes}

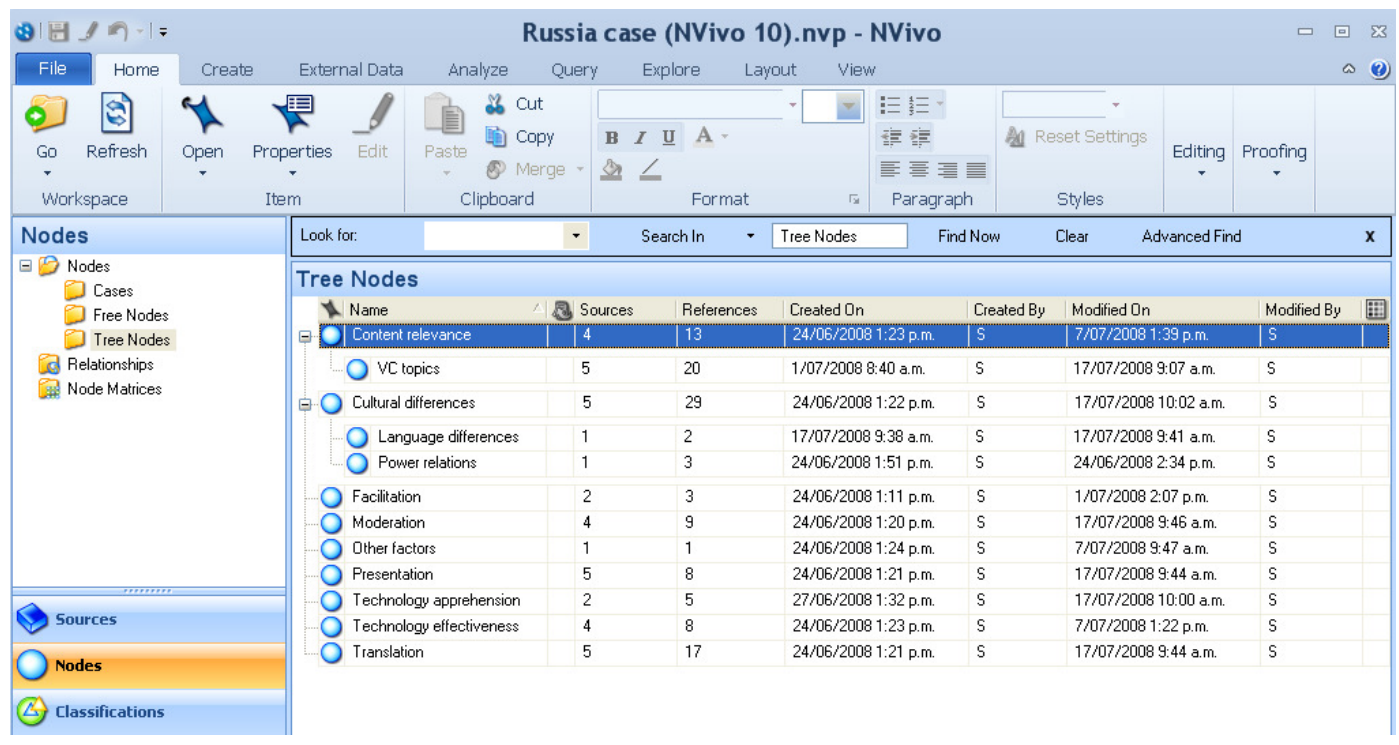

Picture 2: Free nodes

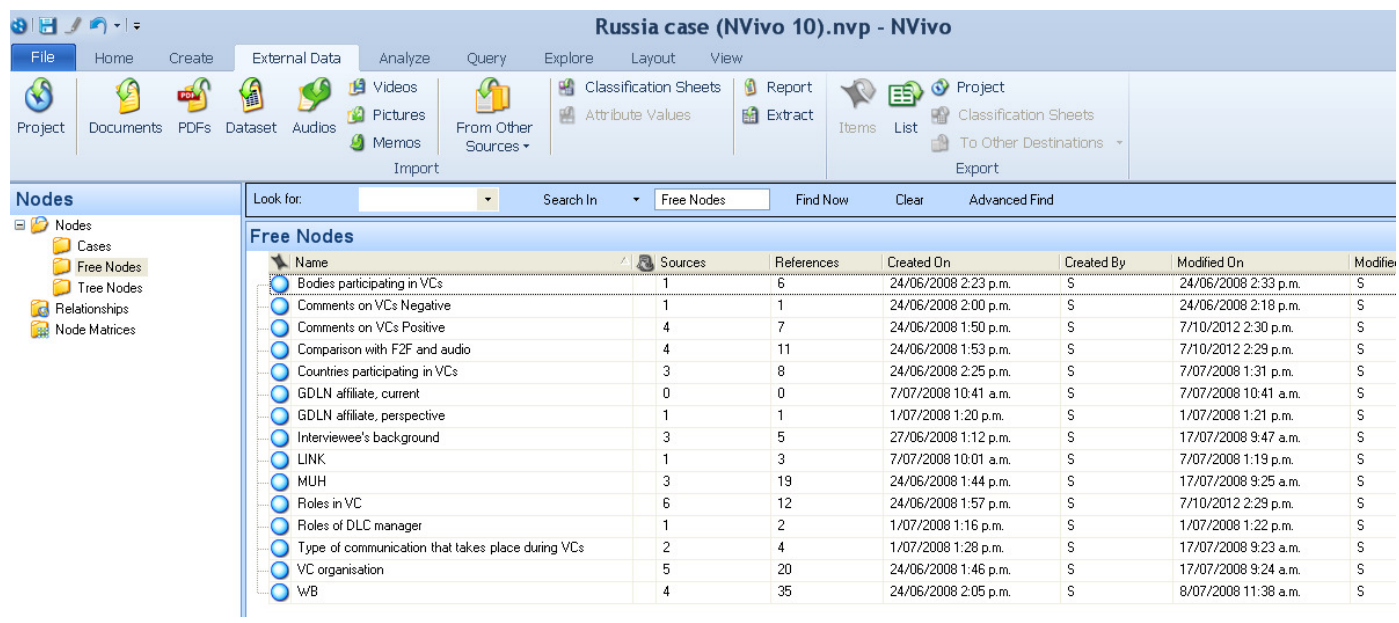




\section{Annex E: Sample table of comparative analysis}

\section{CULTURAL DIFFERENCES: Australla and New Zealand}

\begin{tabular}{|c|c|c|}
\hline & Australia & New Zealand \\
\hline \begin{tabular}{|l} 
Cultural diversity \\
\end{tabular} & $\begin{array}{l}\text { - viewed the diversity as an opportunity, not an obstacle } \\
\text { - VC technology facilitated effective cross-cultural communication } \\
\text { and diminished cultural bariers } \\
\text { - VC technology enriches quality of dialogue } \\
\text { - During VC people engage in communication without being } \\
\text { concemed about cultural differences } \\
\text { - cultural differences, I don't think that affects much. Because } \\
\text { nowadays people are globalised }\end{array}$ & $\begin{array}{l}\text { - It was quite evident, the differences in both the general culture and the workplace culture } \\
\text { - appreciation that the problems they're facing are being faced by others. So that in itself is } \\
\text { actually worth the forum } \\
\text { - experienced professionals or academics, so they were all relatively familiar with the } \\
\text { topics and in that sense I think it reduced the cultural boundaries } \\
\text { - the discussion was dominated by a few people, mainly of European descent, whereas the } \\
\text { participants from Pacific nations kept rather quiet } \\
\text { - New Zealand as a nation, we've come quite a long way in terms of cultural and racial } \\
\text { difference, we've got something to offer in relation to that understanding } \\
\text { - If people aren't used to taking cultural differences into account in relation to a } \\
\text { communication style or the way a leaming situation is approached then their usual } \\
\text { practice will be brought forward into a VC situation. You'll get exactly the same dynamic, } \\
\text { only worse. } \\
\text { - Culture did not really enter into that in a sense of culture derived from ethnicity.... and } \\
\text { there were no obvious specific cultural barriers to communication in that case }\end{array}$ \\
\hline Power distance & $\begin{array}{l}\text { - openness of discussion and trustworthiness of the statements } \\
\text { being made. Some people might feel obliged to express } \\
\text { opinions that fit the viewpoint of their more senior colleagues - a } \\
\text { result of the subordinate's fear of disagreeing with superiors (G. } \\
\text { Hofstede, 2001) } \\
\text { - the minister appeared... so, everybody was rapidly tailoring } \\
\text { their opinions to what they were going to say }\end{array}$ & $\begin{array}{l}\text { - Kept quiet - it might have been respect for a teacher or an older, more authoritative figure } \\
\text { - Because communication is coming ... from a large country, from people using really } \\
\text { technical language... And it has the feeling that a larger country is telling us: "Here's what } \\
\text { - Te should do." } \\
\text { - There's a friendly rivaly, certainly, there's also a senæe in which it's a big country and we, } \\
\text { in New Zealand at least, don't like being told what to do } \\
\text { - "well, I don't want to say too much because l'm not really comfortable with the people l'm } \\
\text { in the room with'. They may not feel they can trust them entirely. } \\
\text { - often get a distinction between what people feel obliged to say, not [what] they actually } \\
\text { think. } \\
\text { - she had very strong views about the project. But sitting in the company of her regional } \\
\text { director in Bangkok. it was very hard to get her to say arything }\end{array}$ \\
\hline
\end{tabular}

\section{CULTURAL DIFFERENCES: Russla and Mongolla}

\begin{tabular}{|c|c|c|}
\hline & Russia & Mongolia \\
\hline \begin{tabular}{|l} 
Cultural diversity \\
\end{tabular} & $\begin{array}{l}\text { - Northern Caucasus. They have a very unique culture which is } \\
\text { different from the rest of Russia. In general, they are quite } \\
\text { explicit on something they don't like ... have no hesitation in } \\
\text { expressing his'her own idea } \\
\text { - cultural differences can be evident when people illustrate their } \\
\text { talk by examples from their rown cultures which might not have } \\
\text { the same meaning or implication for people from other } \\
\text { backgrounds } \\
\text { - unbiased dialogue among peers who were interested in } \\
\text { exchanging professional experience and knowledge } \\
\text { - people in Russia and CIS countries share the same mindset } \\
\text { which is different from a western' mentality. However, a } \\
\text { difference of mentalities is less obvious when cross-cultural } \\
\text { communication irvolves professionals and is focused on a } \\
\text { concrete subject of mutual interest. }\end{array}$ & $\begin{array}{l}\text { - Cultural differences cannot be seen if they can clearly articulate their questions in English } \\
\text { and people understand them } \\
\text { - [in a VC] the variation of these differences is relatively low. Because I think that on the } \\
\text { screen, in the camera, people control themselves more } \\
\text { - experiences of different cultures rather than cultural differences will have an impact [on } \\
\text { communication]. The differences [of cultures] will not be evident because everybody comes } \\
\text { with one mind to get the best solution } \\
\text { - good will. They come to help each other, not to compete } \\
\text { - large automated livestock farms vs. nomadic culture [pastoral husbandir]. We manage } \\
\text { food production and food safety very differently. } \\
\text { - Participants' knowledge on a particular subject expanded as countries shared their } \\
\text { experiences. From this perspective, differences of cultures are beneficial } \\
\text { - the impression that in some countries certain people were assigned in advance to ask } \\
\text { questions whereas in other countries the participants acted spontaneously and just spoke } \\
\text { - participants in Asian countries displayed different attitudes towards the leaming process } \\
\text { - when people from different countries regularty communicated on particular topics, they } \\
\text { started to speak a common professional language no matter what national culture they } \\
\text { came from } \\
\text { - In general, the Asians have similar emotions, resemblance in terms of culture. Perhaps if } \\
\text { we were mixed with the Americans or the Europeans then probably [cultural differences] } \\
\text { would have been more evident. } \\
\text { - It's interesting to watch how people communicate. Some are polite whereas some laugh } \\
\text { offhandedly and behave in "that's us" manner... Some people set good examples but } \\
\text { watching others you get the impression "this is wrong,you shouldn't do that". }\end{array}$ \\
\hline Power distance & $\begin{array}{l}\text { - there was no domination, no superiority of any one country over } \\
\text { another but a very equal discussion was going on } \\
\text { - There was a bit of a tense atmosphere during VCs because } \\
\text { participants here in Moscow were bosses whereas at the other } \\
\text { sites were their subordinates... Maybe because it was a VC, } \\
\text { people in the regions didn't feel pressured by Moscow. It was } \\
\text { more open than a conversation that would've taken place if } \\
\text { these officials were called to come to the ministry office in } \\
\text { Moscow and talk face-to-face. }\end{array}$ & $\begin{array}{l}\text { - they have never demonstrated any cultural differences, something like 'I'm from a superior } \\
\text { nation, you're from a poor nation' } \\
\text { - Power difference was obvious: the WB experts have a tendency to guide, direct and tell } \\
\text { what they have to tell. }\end{array}$ \\
\hline
\end{tabular}

